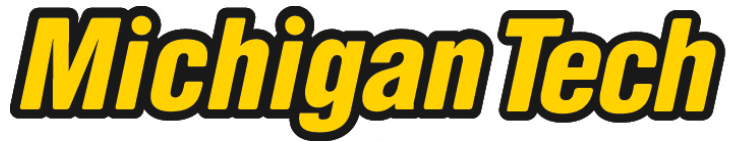 \\ Michigan Technological University Create the Future Digital Commons @ Michigan Tech
}

Dissertations, Master's Theses and Master's Reports - Open

Dissertations, Master's Theses and Master's

Reports

2012

\section{Vortex Shedding Dynamics in Long Aspect-Ratio Aerodynamics Bodies}

Liu Chen

Michigan Technological University

Follow this and additional works at: https://digitalcommons.mtu.edu/etds

Part of the Mechanical Engineering Commons

Copyright 2012 Liu Chen

\section{Recommended Citation}

Chen, Liu, "Vortex Shedding Dynamics in Long Aspect-Ratio Aerodynamics Bodies", Dissertation, Michigan Technological University, 2012.

https://doi.org/10.37099/mtu.dc.etds/473

Follow this and additional works at: https://digitalcommons.mtu.edu/etds

Part of the Mechanical Engineering Commons 


\title{
VORTEX SHEDDING DYNAMICS IN LONG ASPECT-RATIO AERODYNAMICS BODIES
}

\author{
By \\ Liu Chen \\ A DISSERTATION \\ Submitted in partial fulfillment of the requirements for the degree of \\ DOCTOR OF PHILOSOPHY \\ (Mechanical Engineering - Engineering Mechanics) \\ MICHIGAN TECHNOLOGICAL UNIVERSITY \\ 2012
}

(c) 2012 Liu Chen 
This dissertation, "Vortex Shedding Dynamics in Long Aspect-Ratio Aerodynamics Bodies " is hereby approved in partial fulfillment for the requirements for the Degree of DOCTOR OF PHILOSOPHY IN MECHANICAL ENGINEERING - ENGINEERING MECHANICS.

Department of Mechanical Engineering - Engineering Mechanics

Advisor:

Dr. Fernando L. Ponta

Department Chair:

Dr. William W. Predebon

Date: 
To my husband, James A. Peitzmeier \& to my daughter Iris G. Peitzmeier. 


\section{Contents}

List of Figures . . . . . . . . . . . . . . . . . . . . xiii

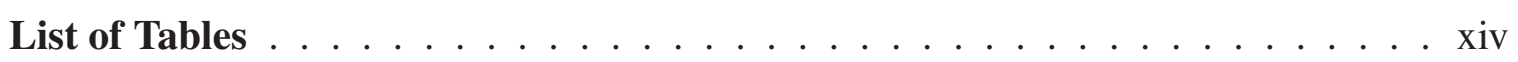

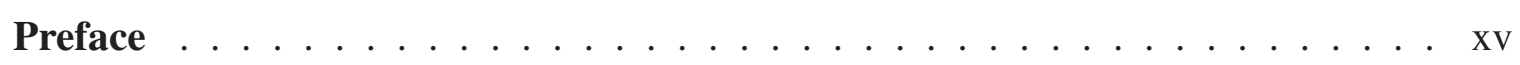

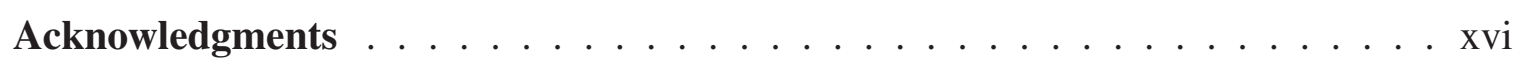

List of Symbols . . . . . . . . . . . . . . . . . . . . . . . . . . . . . .

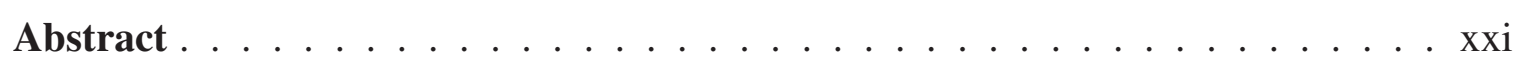

1 Introduction $\ldots \ldots \ldots \ldots \ldots \ldots$

1.1 Fluid structure interaction . . . . . . . . . . . . . 1

1.2 An emerging field of application .............. 3

1.2.1 The wind power challenge ................. 4

1.2.2 The particular problem of floating foundations ...... 7

1.2.3 Wind-farm array efficiency ................... 9 
1.2.4 Ocean energy and hydrokinetic turbines ........... 10

1.3 Dissertation goals . . . . . . . . . . . . . . . 13

1.4 Dissertation outline . . . . . . . . . . . . . . . . 14

2 Vortex-shedding and wake dynamics behind bluff bodies . . . . . . . . 16

2.1 The Kármán vortex street: a classic case-study . . . . . . . . . . . . . 16

2.1.1 The study of the velocity field using Helmholtz decomposition and the evolution of eddy structures ............. 18

2.1.2 Vorticity profiles and decay law of the eddy structures in the vortex

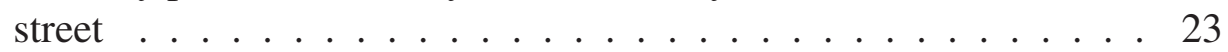

2.2 Wake behind oscillating cylinders ................... 36

2.2.1 Boundaries marking vortex synchronization regions . . . . . . 37

2.2.2 On P+S wakes forming at low Reynolds number ........ 45

2.2.3 Secondary vortex streets and complex wakes ........ 50 50

3 Numerical solution of unsteady-flow problems ............. . . . 55

3.1 Introduction of the Navier-Stokes equations $\ldots \ldots \ldots$

3.2 Numerical Solution of the incompressible viscous flow ........ 56

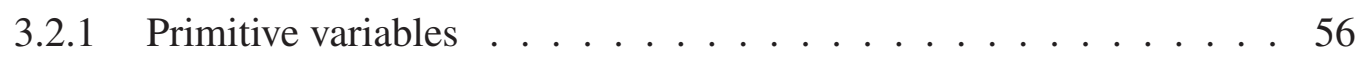

3.2.1.1 Non-fractional step methods .......... . 58

3.2.1.2 Fractional step methods ............. 60 60 
3.2.1.3 Artificial incompressibility .......... 62

3.2.2 Non-Primitive variables . . . . . . . . . . . . . 66 63

3.2.2.1 The Vorticity-Stream Function formulation for 2-D flows 64

3.2.2.2 Biharmonic formulation . . . . . . . . . . 65

3.2.2.3 Coupled formulation in Vorticity and Stream Function . . 66

3.2.2.4 Uncoupled formulation using vorticity integral conditions 66

4 The Hybrid-Method Family and the KLE . . . . . . . . . . . . . . . 69

4.1 Hybrid methods . . . . . . . . . . . . . . . . . . . . . . 69

4.1.1 Hybrid methods in three dimensions . . . . . . . . . . . . 71

4.1.2 Equations in two dimensions . . . . . . . . . . . . 72

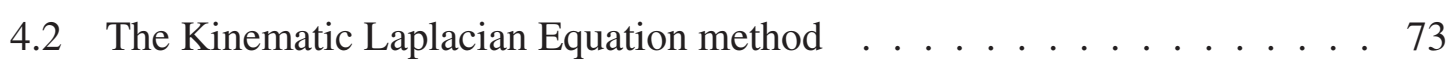

4.2.1 The Constant-Curl Laplacian Equation $\ldots . . . . . . . .73$

4.2.2 A generalized Laplacian $(\boldsymbol{\omega}, \mathbf{v})$ method: The KLE $\ldots \ldots . . .73$

4.2.3 Variational formulation of the KLE method . . . . . . . . 76

4.3 Numerical implementation of the KLE method . . . . . . . . . 78

4.3.1 The Spectral-element method for KLE . . . . . . . . . . . . . 80

5 Numerical experimentation . . . . . . . . . . . . . . . . 88

5.1 Wake dynamics behind bluff bodies at low Re . . . . . . . . . 88 
5.2 Decay of wake oscillations at low Re numbers . . . . . . . . . . 93

5.2.1 Numerical results ....................... 94

5.2.2 Theoretical Low-dimensional Model . . . . . . . . . . . . . . . . 113

$5.3 \mathrm{Re}-\mathrm{St}$ relationship . . . . . . . . . . . . . . . . 117

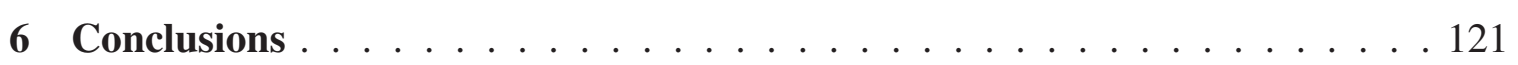

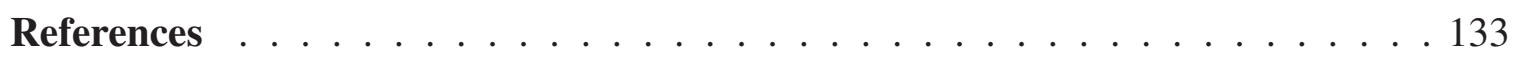

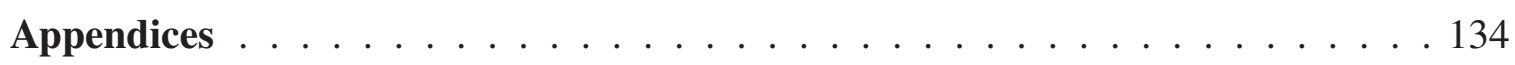

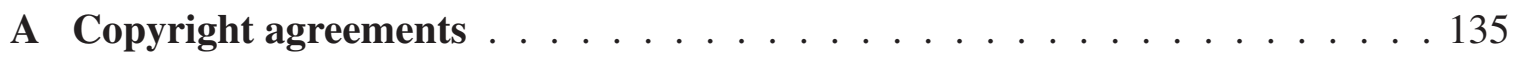

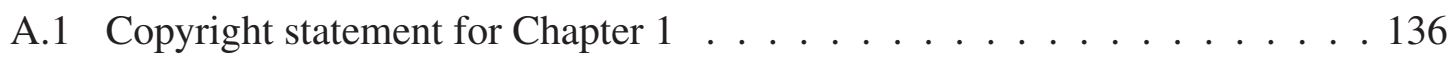

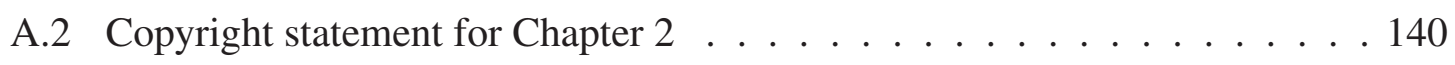




\section{List OF Figures}

1.1 The wind-turbine upscaling phenomenon, [taken from DOE report 1 ] . . . . . 5

1.2 The REpower M5 5-megawatt turbine, with a rotor diameter of approximately 126 meters. . . . . . . . . . . . . . 6 6

1.3 Alternative concepts for floating foundations for deep-water offshore Wind Turbines, [taken from NREL report 2] . . . . . . . . . . . . 8 8

2.1 A Tri-quadrilateral finite element mesh used in a study by Ponta (lengths in diameters of the circular cylinder), [Figure created by author from data

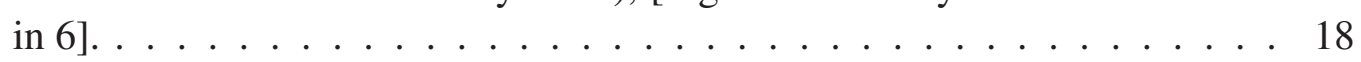

2.2 For $R e=140$, the rearrangement of the eddies during one vortex-shedding cycle in the wake behind a circular cylinder is shown using the vorticity isolines and the pattern of streamlines of $\boldsymbol{v}_{\omega}$ [Figure created by author from data in 6]. Sequence should be followed clockwise. . . . . . . . . . . . . 19

2.3 For $R e=140$, the vortex-formation during one vortex-shedding cycle in the wake behind a circular cylinder is shown using vorticity isolines and the pattern of streamlines of $\boldsymbol{v}_{\omega}$, [Figure created by author from data in 6]. . 20]

2.4 Comparison of the numerical Strouhal number using the KLE method with Williamson's experimental measurements [7] for $50<R e<180$. . . . . . 21

2.5 For $\mathrm{Re}=100$, upper panel shows the superimposition of vorticity isolines with pattern of streamlines of $\boldsymbol{v}$, while the lower panel presents the superimposition of vorticity isolines $\boldsymbol{v}_{\omega}$ [Figure created by author from data in

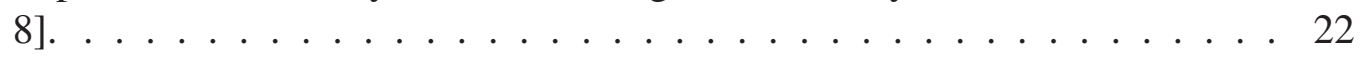


2.6 Panel (a): Three-dimensional perspective view of the vorticity distribution of a twelve-vortex series in a Kármán-street wake for $\operatorname{Re}=100$. Panel $(b)$ : Lateral view of the same vorticity distribution, [Figure created by author from data in 8$] . \ldots \ldots \ldots 26 . \ldots \ldots$

2.7 Elliptical ratio for several plane sections of the vortex core corresponding to horizontal slicing planes located at $0.6,0.7,0.8$ and 0.9 of $\omega_{p}$ and best-fit line, [Figure created by author from data in 8] . . . . . . . . . . 2 27

2.8 Decay of $\omega_{p}^{*}$ along the streamwise axis and best-fit hyperbola $\omega_{p}^{*}=$ $19.579\left(x^{*}+5.259\right)^{-1}$, [Figure created by author from data in 8] . . . . . 29

2.9 Panel (a): Comparison of the vorticity profiles in a cross-flow slicing plane for ten consecutive vortices. $y^{*}$ indicates, as hitherto, the non-dimensional cross-flow distance taken from the origin. Panel $(b)$ : Comparison of the vorticity profiles in a streamwise slicing plane for ten consecutive vortices. $x_{v c}^{*}$ indicates the non-dimensional streamwise distance taken from the vortex centre, [Figure created by author from data in 8] . . . . . . . . . . 31

2.10 Three-dimensional perspective view of the $\psi_{\omega}$ distribution corresponding to the Kármán-street wake depicted in figure 2.6, [Figure created by author from data in 8$] \ldots \ldots \ldots$

$2.11 \omega^{*}-\psi_{\omega}^{*}$ scatter plot for a complete ten-vortex wake at $R e=100$, [Figure created by author from data in 8$]. \ldots$. . . . . . . . . . . . 34

$2.12 \omega^{*}-\psi_{\omega}^{*}$ scatter plots for the first and the third of the five vortex pairs in the wake depicted in figure 2.11, [Figure created by author from data in 8]. The solid lines correspond to the theoretical $\omega-\psi$ curve for a Lamb vortex located at the same distance downstream. . . . . . . . . 35

2.13 Original $(\lambda / D, A / D)$ map [9] with the numerical $0.001,0.015$ and 0.06 isolines of $\Delta(a) R e=392(b) R e=140$. The Points of the parameters used in the numerical experiments are also shown in (b), [taken from 10]. See appendix for permission. . . . . . . . . . . . . 41

2.14 The sinusoidal trajectory of the oscillating cylinder, [Figure created by author from data in 11]. . . . . . . . . . . . . . . 42 
2.15 (a), Vortex synchronization regions for cylinders oscillating normally in the plane represented by amplitude and wavelength plane as seen in experiments by Williamson and Roshko [9], see appendix for permission, $(b)$, synthetic version of the WR map with boundaries defined by the $L_{D}$ and $\Delta$ isolines, [Figure created by author from data in 11] . . . . . . . . . 44

2.16 Vorticity field shown in gray scale as obtained by KLE at $R e=140$ compared with a flow visualization by $\mathrm{C}$. H. K. Williamson(see permission in appendix), of a $\mathrm{P}+\mathrm{S}$ wake of an oscillating cylinder for the same Re, [Figure created by author from data in 10] . . . . . . . . . . 46

2.17 Non-dimensional vorticity field obtained by KLE for a $\mathrm{P}+\mathrm{S}$ wake with $R e=140, \lambda / D=7.5$, and $A / D=1$, [Figure created by author from

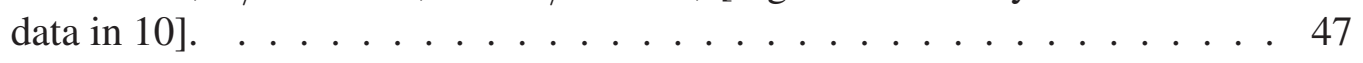

2.18 plot showing the sequence of events of the vortex splitting process producing a $\mathrm{P}+\mathrm{S}$ wake starting from the bottom left panel moving clockwise, [Figure created by author from data in 10] . . . . . . . . . . . 48

2.19 Non-dimensional vorticity field for a weak $\mathrm{P}+\mathrm{S}$ wake with $R e=140$ $(\lambda / D=8.5, A / D=1)$, [Figure created by author from data in 10]. . . . 49

2.20 Non-dimensional vorticity field for a chaotic wake with $R e=140(\lambda / D=$ $8.5, A / D=0.3)$, [Figure created by author from data in 10] . . . . . . 4 49

2.21 (a), Path followed by the cylinder's center at a constant speed for $A 1$ $(\lambda / D=7.5, A / D=1)$. $(b)$, Path of the cylinder when the reference frame moves horizontally with speed $U_{x m}$. [Figure created by author from

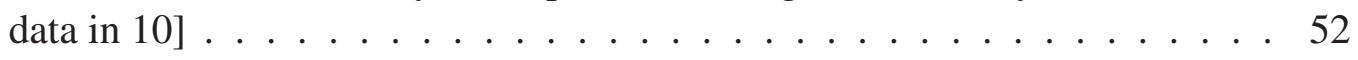

2.22 Non-dimensional vorticity field obtained by KLE for the hyper-Kármán vortex-street with $R e=140, \lambda / D=7.5$, and $A / D=1$, [Figure created by author from data in 10$]. \ldots \ldots \ldots 5$

2.23 plot depicting the vortex-merging mechanism producing the hyperKármán vortex-street, [Figure created by author from data in 10]. The sequence starts at the bottom left panel moving clockwise. . . . . . . . 54 
4.1 A two-dimensional nine node isoparametric element in its natural coordinate system along with a graphical representation of three of its nine interpolation functions i.e nodes 3,8 and 9 , [Figure created by author from data in 6 ]. . . . . . . . . . . . . . . . . . . . . . . . . . .

4.2 A tri-quadrilateral finite element mesh derived from an unstructured triangular mesh, [Figure created by author from data in 6] . . . . . . . . . . . 84

4.3 The internal topology of a tri-quadrilateral element. Quadrilateral elements (I)-(III) are the nine-node isoparametric elements, [Figure created by author from data in 6]. 1-19 is the in-triangle global numbering of the nodes. . 85

5.1 Closed near-wake behind circular cylinder at $\mathrm{Re}=28.4$ [taken from 12]. . . 90

5.2 Closed near-wake behind circular cylinder at $\mathrm{Re}=41$ [taken from 12] . . . 90

5.3 Comparison of the wake length computed numerically with Taneda's experimental data. . . . . . . . . . . . . . . . . . . . . . 91

5.4 The unstructured triangular mesh used in KLE evaluation. . . . . . . . . . 94

5.5 The closer view of the mesh above. . . . . . . . . . . . . . . 94

5.6 The modified velocity boundary profile. . . . . . . . . . 95

5.7 Comparison of numerical results and theoretical model for wake oscillation at $\mathrm{Re}=30$ : $(a)$, probe locates at 1.3(cylinder diameter) downstream; $(b)$, probe locates at 16(cylinder diameter) downstream; $(c)$, probe locates at 28(cylinder diameter)

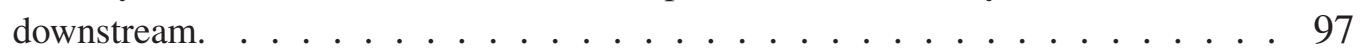

5.8 Comparison of numerical results and theoretical model for wake oscillation at $\operatorname{Re}=31:(a)$, probe locates at 1.3(cylinder diameter) downstream; $(b)$, probe locates at 16(cylinder diameter) downstream; $(c)$, probe locates at 28(cylinder diameter) downstream. ......................... 98

5.9 Comparison of numerical results and theoretical model for wake oscillation at $\operatorname{Re}=32:(a)$, probe locates at 1.3(cylinder diameter) downstream; $(b)$, probe locates at 16(cylinder diameter) downstream; $(c)$, probe locates at 28(cylinder diameter) downstream. . . . . . . . . . . . . . . . 999 
5.10 Comparison of numerical results and theoretical model for wake oscillation at $\operatorname{Re}=33:(a)$, probe locates at 1.3(cylinder diameter) downstream; $(b)$, probe locates at 16(cylinder diameter) downstream; $(c)$, probe locates at 28(cylinder diameter) downstream. . . . . . . . . . . . . . . . . . . 100

5.11 Comparison of numerical results and theoretical model for wake oscillation at $\operatorname{Re}=34:(a)$, probe locates at 1.3(cylinder diameter) downstream; $(b)$, probe locates at 16(cylinder diameter) downstream; $(c)$, probe locates at 28(cylinder diameter) downstream. . . . . . . . . . . . . . . . . 101

5.12 Comparison of numerical results and theoretical model for wake oscillation at $\operatorname{Re}=35:(a)$, probe locates at 1.3(cylinder diameter) downstream; $(b)$, probe locates at 16(cylinder diameter) downstream; $(c)$, probe locates at 28(cylinder diameter) downstream. . . . . . . . . . . . . . . 102

5.13 Comparison of numerical results and theoretical model for wake oscillation at $\operatorname{Re}=36:(a)$, probe locates at 1.3(cylinder diameter) downstream; $(b)$, probe locates at 16(cylinder diameter) downstream; $(c)$, probe locates at 28(cylinder diameter) downstream. . . . . . . . . . . . . . . . . 103

5.14 Comparison of numerical results and theoretical model for wake oscillation at $\operatorname{Re}=37:(a)$, probe locates at 1.3(cylinder diameter) downstream; $(b)$, probe locates at 16(cylinder diameter) downstream; $(c)$, probe locates at 28(cylinder diameter) downstream. . . . . . . . . . . . . . . . . . . 104

5.15 Comparison of numerical results and theoretical model for wake oscillation at $\operatorname{Re}=38:(a)$, probe locates at 1.3(cylinder diameter) downstream; $(b)$, probe locates at 16(cylinder diameter) downstream; $(c)$, probe locates at 28(cylinder diameter) downstream. . . . . . . . . . . . . . . . . 105

5.16 Comparison of numerical results and theoretical model for wake oscillation at $\operatorname{Re}=39:(a)$, probe locates at 1.3(cylinder diameter) downstream; $(b)$, probe locates at 16(cylinder diameter) downstream; $(c)$, probe locates at 28(cylinder diameter) downstream. . . . . . . . . . . . . . . . . . 106

5.17 Comparison of numerical results and theoretical model for wake oscillation at $\operatorname{Re}=40:(a)$, probe locates at 1.3(cylinder diameter) downstream; $(b)$, probe locates at 16(cylinder diameter) downstream; $(c)$, probe locates at 28(cylinder diameter) downstream. . . . . . . . . . . . . . . 107 
5.18 Comparison of numerical results and theoretical model for wake oscillation at $\operatorname{Re}=41:(a)$, probe locates at 1.3(cylinder diameter) downstream; $(b)$, probe locates at 16(cylinder diameter) downstream; $(c)$, probe locates at 28(cylinder diameter) downstream. . . . . . . . . . . . . . . . . . . . . 108

5.19 Comparison of numerical results and theoretical model for wake oscillation at $\operatorname{Re}=42:(a)$, probe locates at 1.3(cylinder diameter) downstream; $(b)$, probe locates at 16(cylinder diameter) downstream; $(c)$, probe locates at 28(cylinder diameter) downstream. . . . . . . . . . . . . . . . . . . . 109

5.20 Comparison of numerical results and theoretical model for wake oscillation at $\mathrm{Re}=43:(a)$, probe locates at 1.3(cylinder diameter) downstream; $(b)$, probe locates at 16(cylinder diameter) downstream; $(c)$, probe locates at 28(cylinder diameter) downstream. . . . . . . . . . . . . . . . 110

5.21 Comparison of numerical results and theoretical model for wake oscillation at $\operatorname{Re}=44:(a)$, probe locates at 1.3(cylinder diameter) downstream; $(b)$, probe locates at 16(cylinder diameter) downstream; $(c)$, probe locates at 28(cylinder diameter) downstream. . . . . . . . . . . . . . . . . 111

5.22 Rate of decay at the closed near-wake for $\mathrm{Re}=30-44$. . . . . . . . . . 113

5.23 Twin-T filter circuit. . . . . . . . . . . . . . . . . . . 115

5.24 Vortex Oscillator model. . . . . . . . . . . . . . . . . . 117

5.25 Diagram of the vortex oscillator model. . . . . . . . . . . 117

5.26 Comparison of StRe vs Re with Camichel's experiment at $R e=16-47$. 120 


\section{LIST OF TABLES}

5.1 Amplitude $B$ at different probes locations $x \ldots \ldots \ldots \ldots$ 


\section{PREFACE}

The dissertation presents my research work in pursuing the Ph.D. degree in Mechanical Engineering-Engineering Mechanics at Michigan Technological University. It contains previously published journal articles in Chapter 1 . Chapter 5 contains original work which is in preparation for publication.

Chapter 1 contains excerpts of two articles previously published in the journal of Energy for Sustainable Development. Here, the individual contribution to the previously published papers is briefly summarized. As the first author in the article, "Perspectives on innovative concepts in wind-power", I presented a review on the innovation in the development of wind power. The article was written by me, and revised by Lucas Lago with the advice from F. L. Ponta. For the article "Advances and trends in hydrokinetic turbine systems", it was written by me, joint effort from Lucas Lago and me for the idea and structure for the article. The completion of this article was also advised by F. L. Ponta. 


\section{ACKNOWLEDGMENTS}

It would not have been possible for me to finish the dissertation without helps from my committee members, and support from my family and friends.

Firstly, I would like to express my deepest gratitude to Dr. Fernando Ponta, my advisor, for providing me with an excellent atmosphere in research, his excellent guidance, caring, patience through these years. Special thanks goes to him for the support and understanding during the pregnancy with my daughter.

I would like to thank my parents, younger sister and younger brother. They were always supporting me and encouraging me with their best wishes.

I would like to thank my husband, James Peitzmeier. He was always there cheering me up and stood by me through the good times and bad. And I would like to thank my daughter, Iris Peitzmeier, the precious in my life.

I would like to thank the whole research group at MTU, Lucas Lago, Anurag Rajan and Pankaj Jagadale, Dr. Alejandro Otero, who as good friends, were always willing to help and give their best suggestions.

Many thanks to the people I met at MTU for the times we spent together and for friendship. To Qili Hu, Jie Tang, Zheng Zhang, Xiaobo Song, Nick Riegel, Marisa Bertuzzi, Edward $\mathrm{Ng}$, Suting Lau, Kenny Ng, Shuting Goh, and my friends back in China.

Finally, I would like to thank Michigan Technological University for the opportunity and the support, and also to the U. S. National Science Foundation for the support though the grants CEBET-0933058 and CEBET-0952218. 


\section{LiST OF SYMBOLS}

\section{Symbols in Chapter 1}

$D_{r} \quad$ rotor diameter of wind turbine

\section{Symbols in Chapter 2}

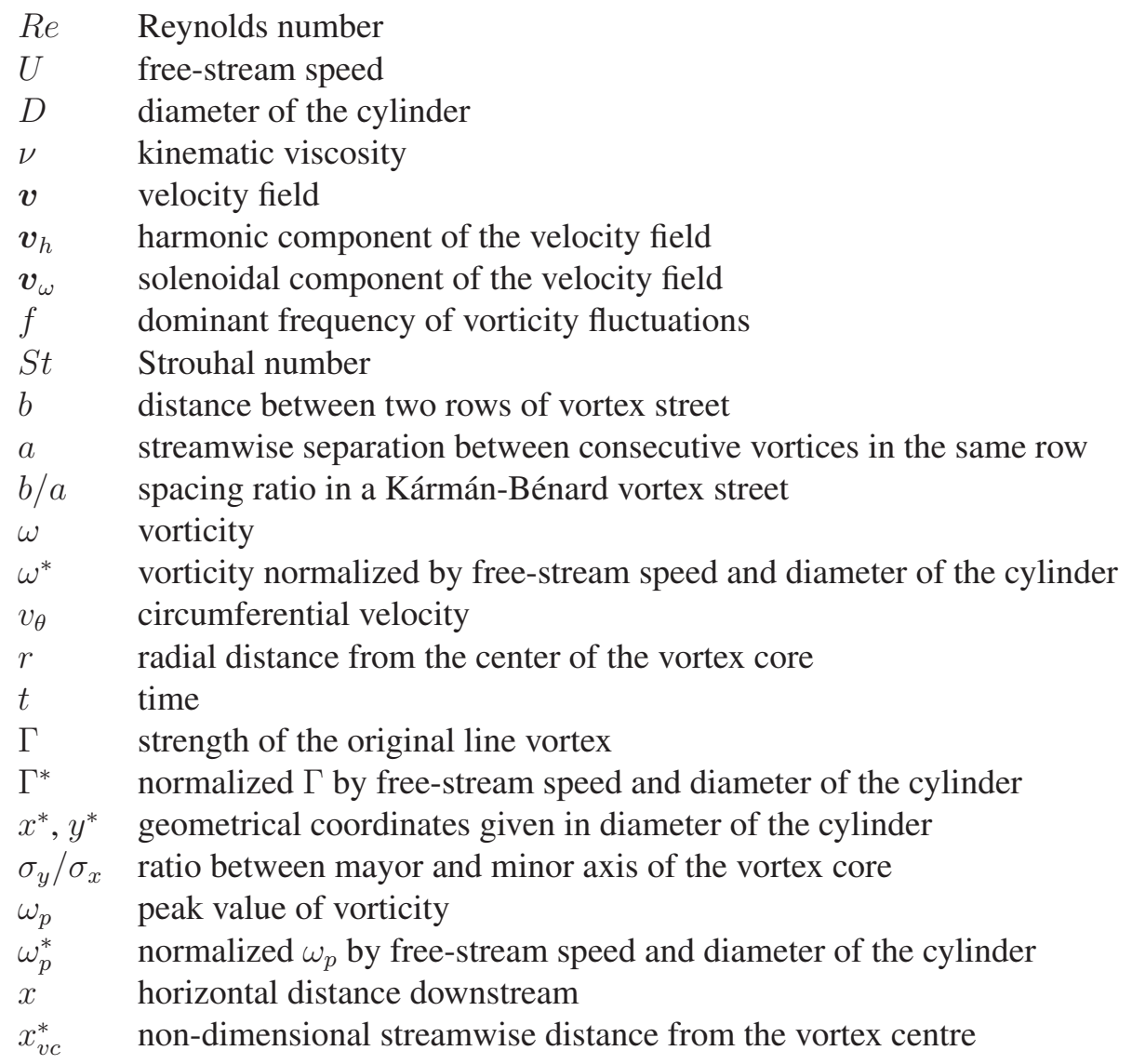




$\begin{array}{ll}x_{0} & \text { a virtual point upstream of the cylinder } \\ x_{0}^{*} & \text { normalized } x_{0} \text { by diameter of the cylinder } \\ U_{w} & \text { speed of propagation of the wake } \\ U_{w}^{*} & \text { normalized } U_{w} \text { by free-stream speed } \\ R & \text { equivalent radius } \\ \psi_{\omega} & \text { stream function } \\ \psi_{\omega}^{*} & \text { normalized } \psi_{\omega} \text { by free-stream speed and diameter of the cylinder } \\ \psi_{\omega} & \text { stream vector potentials associated with } \boldsymbol{v}_{\omega} \\ \psi_{h} & \text { stream vector potentials associated with } \boldsymbol{v}_{h} \\ \psi_{\omega} & \text { 2-D stream function corresponding to } \boldsymbol{\psi}_{\omega} \\ \psi_{h} & \text { 2-D stream function corresponding to } \boldsymbol{\psi}_{h} \\ \left.U_{x}, U_{y}\right) & \text { translational velocity of the dipole } \\ \lambda & \text { wavelength of the oscillations of the cylinder } \\ \lambda / D & \text { Non-dimensional wavelength of the oscillations of the cylinder } \\ A & \text { amplitude of the oscillations of the cylinder } \\ A / D & \text { Non-dimensional amplitude of the oscillations of the cylinder } \\ T_{e} & \text { period of the forced oscillation } \\ T_{s} & \text { instantaneous period of vortex shedding for a non-oscillating cylinder } \\ \hat{T}_{s} & \text { shedding period } \\ \hat{S} t & \text { Strouhal number associated with } \hat{R} e \\ \hat{t} & \text { non-dimensional time } \\ U_{t g} & \text { tangential speed of the cylinder } \\ U_{x, m} & \text { average horizontal speed } \\ \lambda_{g} & \text { average of horizontal speed }\end{array}$




\section{Symbols in Chapter 3}

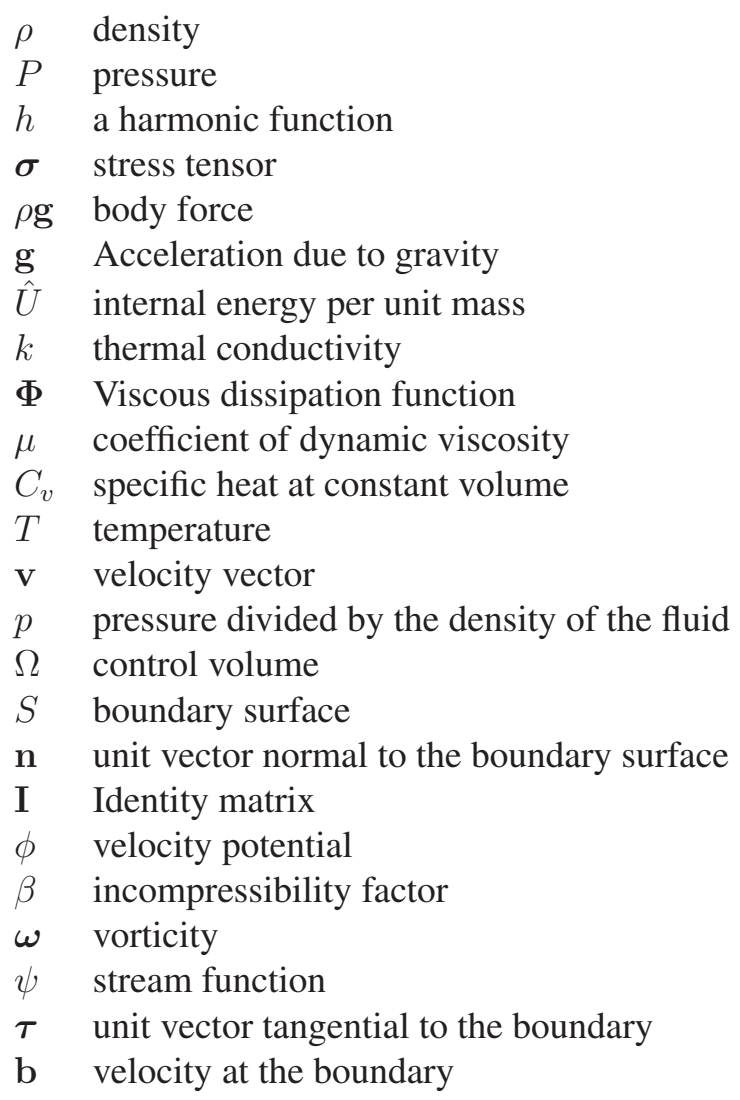

\section{Symbols in Chapter 4}

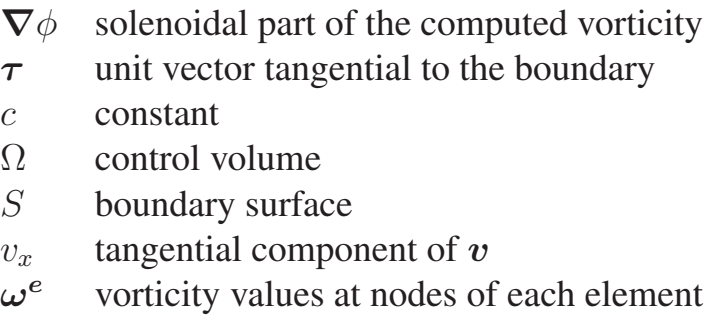




\section{Symbols in Chapter 5}

$R e_{o s c} \quad$ Reynolds number at which oscillations start

$R e_{s} \quad$ separation Reynolds number

$R e_{v s} \quad$ Reynolds number at which a vortex street begins to shed

$E \quad$ width of the confining walls of the windwater tunnel

$L_{w} \quad$ length of the closed near-wake

$u_{\max } \quad$ maximum reverse velocity

$L_{w l} \quad$ wave-length of trail oscillation

$u \quad$ the wave speed

$\omega_{a n} \quad$ the analytical vorticity

$B \quad$ amplitude of the wake oscillation

$D_{W} \quad$ decay of the wake oscillation

$T_{t} \quad$ time span in which the wake oscillates

$T_{\text {ave }} \quad$ average frequency of the wake oscillation

$S_{1} S_{2} \quad$ power source

$C_{1}-C_{4}$ capacitors

$f \quad$ shedding frequency of the wake

Ro Roshko number 


\title{
Vortex Shedding Dynamics in Long Aspect-Ratio Aerodynamics Bodies
}

\author{
Liu Chen \\ Michigan Technological University, 2012 \\ Advisor: Dr. Fernando L. Ponta
}

\begin{abstract}
The focus of the current dissertation is to study qualitatively the underlying physics of vortex-shedding and wake dynamics in long aspect-ratio aerodynamics in incompressible viscous flow through the use of the KLE method. We carried out a long series of numerical experiments in the cases of flow around the cylinder at low Reynolds numbers. The study of flow at low Reynolds numbers provides an insight in the fluid physics and also plays a critical role when applying to stalled turbine rotors. Many of the conclusions about the qualitative nature of the physical mechanisms characterizing vortex formation, shedding and further interaction analyzed here at low Re could be extended to other $\operatorname{Re}$ regimes and help to understand the separation of the boundary layers in airfoils and other aerodynamic surfaces. In the long run, it aims to provide a better understanding of the complex multi-physics problems involving fluid-structure-control interaction through improved mathematical computational models of the multi-physics process. Besides the scientific conclusions produced, the research work on streamlined and bluff-body condition will also serve as a valuable guide for the future design of blade aerodynamics and the placement of wind turbines and hydrakinetic turbines, increasing the efficiency in the use of expensive workforce, supplies, and infrastructure.
\end{abstract}

After the introductory section describing the main fields of application of wind power and hydrokinetic turbines, we describe the main features and theoretical background of the numerical method used here. Then, we present the analysis of the numerical experimentation results for the oscillatory regime right before the onset of vortex shedding for circular cylinders. We verified the wake length of the closed near-wake behind the cylinder and analysed the decay of the wake at the wake formation region, and then studied the St-Re relationship at the Reynolds numbers before the wake sheds compared to the experimental data. We found a theoretical model that describes the time evolution of the amplitude of fluctuations in the vorticity field on the twin vortex wake, which accurately matches the numerical results in terms of the frequency of the oscillation and rate of decay. We also proposed a model based on an analog circuit that is able to interpret the concerning flow by reducing the number of degrees of freedom. It follows the idea of the non-linear oscillator and resembles the dynamics mechanism of the closed near-wake with a common configured sine wave oscillator. This low-dimensional circuital model may also help to understand the underlying physical mechanisms, related to vorticity transport, that give origin to those oscillations. 


\section{INTRODUCTION}

The flow of fluids can be seen all around us, be it the natural environment or almost any technical field. Meteorological phenomena, combustion processes, HVAC systems, pollution, and the numerous processes in the human body are some examples of fluid flow we encounter almost everyday. The number of applications of fluid flow analysis is enormous: breathing, blood flow, turbines, airplanes, ships, windmills, and engines to name a few, making the analysis of flow one of the most important areas of research in the last half century.

Mathematically, the flow of fluids is represented by the known Navier-Stokes equations, a system of non-linear partial differential equations. These equations describe the conservation laws for mass, momentum, and energy for the flow of fluids concerned. The NavierStokes equations present a challenging problem to mathematicians and engineers in finding solutions related to proof of existence and finding accurate yet efficient numerical methods with proper boundary conditions.

Solving the Navier-Stokes equations numerically is important because it can be used to model many aspects of engineering analysis as well as academic interest. It offers an attractive alternative to the expensive, and sometimes extremely difficult to implement, experimental analysis of flow patterns.

\subsection{Fluid structure interaction}

One of the most important applications of the numerical solutions of these equations would be the numerical modeling of Fluid-Structure Interaction (FSI). The non-linear dynamics involved in such interactions provides insights into numerous engineering problems such as the response of high rise buildings and bridges to strong winds, blood flow through arteries,

The material contained in this chapter is partly published in Energy for Sustainable Development 14 (2010) \& 15 (2011). See Appendix A.1 for a copy of the copyright permission. 
vibrations in turbine blades, aerodynamic response characteristics of aircraft wings, marine hydrodynamics which includes modeling fluid flow interaction with marine systems, offshore and coastal structures, underwater systems and structures, and the ever popular aerodynamic modeling of automobiles. These phenomena manifest themselves at a wide range of scales and present excellent opportunities for scientific discovery with a richness of technical application.

An experimental analysis for some of the FSI problems might not always be the most attractive prospect. The case of a wind turbine is a perfect example because of the enormity of the surfaces involved. The economy of scales factors have driven companies to consider rotors with diameters as large as 200 meters. Extrapolating experimental data from wind tunnels in such cases is very complicated. Another interesting example would be the modeling of bridges and high rise buildings and their interaction with high winds. Such huge structures run the risk of stress-related failures due to FSI, and hence require a very accurate modeling of flow patterns.

On the other extreme, problems of placing sensors on small-scale mechanisms with complex roto-translational motion, like the Micro-Air-Vehicle modelled on insect flight, makes experimental analysis extremely difficult. A numerical scheme seems like an attractive alternative to experimental prototypes and should also help bring down the overall cost. But a numerical solution comes with its own set of problems, starting with complex physics involved in slender-body aeroelastic dynamics. The aeroelastic dynamics in slender bodies depends not only on the characteristic modes of the body structure itself, but also on the amplitude and frequency of the fluctuation of the aerodynamic forces. These forces are strongly affected by the dynamics of the vortex-wake shed from the body, which itself depends on the body's oscillations. Vortex-induced vibration can lead to catastrophic failure of engineering systems, as was clearly illustrated by the Tacoma Narrows Bridge disaster. A resulting periodic vibration essentially depends on the work done by the fluid on the body over a cycle. This is associated with the timing of the vortex dynamics because the induced side force caused by the body motion significantly influences the net energy transfer [13].

A significant challenge in analyzing these systems is the fact that the vortex wake produced by an oscillating body is very different from the classic Kármán vortex street, which would translate into a complex fluctuating aerodynamic force. Many roto-translational mechanisms have a dynamic control system reacting to structural responses to fluid flow as well as varying loads, thereby optimizing efficiency and extending their lifetime. This control system would also have to be incorporated into the numerical model for an accurate representation. The problem is no longer just an unsteady flow simulation, but a multiphysics problem involving non-linear structural dynamics as well as a dynamic control system in addition to the fluid flow model. All of this, combined with the discretization of complex geometries, makes it quite difficult to numerically solve the non-linear Partial Differential Equations (PDEs) involved. Further complications may arise with time- 
marching integration of multiphysics problems. Adaptive variable-timestep/variable-order Ordinary Differential Equations (ODE) algorithms provide a way to improve the efficiency of time-marching schemes. But finding a way to combine those adaptive algorithms with the discretization of the spatial PDE problem has proved to be difficult. An innovative computational scheme to solve these problems was introduced in Ponta [6] known as the Kinematic Laplacian Equation (KLE) method. The KLE method invloves a hybrid formulation of the Navier-Stokes equations using velocity and vorticity as the primary variables, rather than the conventional formulation in terms of pressure and velocity, and is a natural extension of the well-established vorticity-stream function methods. The emergence of vorticity-velocity methods might be considered one of the most recent innovations in the computational solution of time-dependent viscous flows. Even though the appearance of what could be regarded as the first vorticity-velocity approach may be traced as early as 1976 [14], it is only during the last decade or so that a systematic research effort has been applied to the development of this family of methods (see [6.15] for a complete list of references). Compared with the classical formulation on primitive variables (velocity-pressure) or with their vorticity-stream-function cousins, the vorticity-velocity methods present several advantages.

The KLE algorithm solves the vorticity transport equation as an ODE problem in time with input velocity solved from a modified Poisson's equation in velocity, called the Kinematic Laplacian Equation, at each spatial node. The input to solve the KLE is provided by integrating the vorticity at each time step. Thus, it creates an evolving scheme in which the KLE provides the input for the ODE algorithm and vice-versa. Since time is the only iteration variable present, coupling the fluid analysis with other physical mechanisms (e.g. structural response, control-system dynamics, etc.) becomes possible now by increasing the number of the equations to the ODE system. The KLE also shows a substantial advantage when coping with complex geometries because it allows, to a larger extent, the use of unstructured meshes, which gives a more suitable meshing compared with structured-mesh approaches. This is a very convenient feature for dealing with the complex aerodynamic shape of wind-turbine blades, helicopter-rotor blades, insect wings, or other aerodynamic surfaces.

\subsection{An emerging field of application}

This research is a small yet important part of the ongoing work towards the advancement of computational mathematical models for complex multiphysics problems that involves the fluid-structure-control interaction. This interaction makes its appearance in many en-

gineering applications, thus research in this field provides a better understanding of the underlying physics. 


\subsubsection{The wind power challenge}

One such important engineering problem is the harnessing of wind power. The reemergence of wind as a significant energy source is now driven by the need to meet increasing worldwide electricity demand and reduce the environmental impact caused by the conventional electricity generation technologies. Thanks to technological innovations, wind power is not only becoming less expensive on a large scale, but it is also one of the cleanest ways to produce energy. Considerable progress in wind-power technology during the last few decades has made it an important supplier of grid-connected electricity in the big world energy picture. Nowadays, wind power is the most rapidly growing and most widely utilized renewable energy technology, with a total of $159.2 \mathrm{GW}$ installed worldwide at the end of 2009 , producing 340 TWh per year, which is about $2 \%$ of worldwide electricity generation [16].

During the last decade, the global installation of wind power capacity has accelerated. The wind industry mainly concentrates in Europe and the US, but it is emerging in China, India, and Brazil. The global installed capacity grew from 14,604 MW to 84,934 MW in the period of 2000-2007, which represents a compounded annual growth rate of $28.6 \%$, and an impressive rate of $482 \%$ over a period of only seven years [17].

There has been a spontaneous tendency in the wind-turbine industry to increase the size of the state-of-the-art machine [18] and substantially reduce the cost of wind energy, see Figure 1.1. Output power of these turbines range from 3.6 to $6 \mathrm{MW}$, with rotor diameters up to 127 meters, see an example of a kind in Figure 1.2. Next-generation offshore turbines with rotor diameters up to 200 meters have been suggested [19]. The technological challenge in wind power nowadays is to develop a next generation of upscaled low-cost turbines that may further reduce generation costs. If this generation of superturbines is successfully developed, wind-energy costs would be reduced substantially.

Simultaneously, wind turbine design has followed the tendency to go to variable-speed pitch-controlled turbines from fixed-speed stall-controlled turbines [21]. Wind turbines available today show various innovative concepts combined with the verified technology for both power electronics and generators [22]. One way to improve power output and operational flexibility in Horizontal-Axis Wind Turbines (HAWTs) is to shroud the rotor into a divergent duct. This concept is usually referred to as a Diffuser-Augmented Wind Turbine (DAWT). The idea is to induce mass flow augmentation and increase power extraction by accelerating the flow inside the duct and converting the downstream flow's kinetic energy into a pressure drop behind the rotor, which makes it possible to capture the airflow from a stream tube which has greater area compared to that of the rotor itself [23].

Nevertheless, several technological challenges remain to develop wind power. Some are 


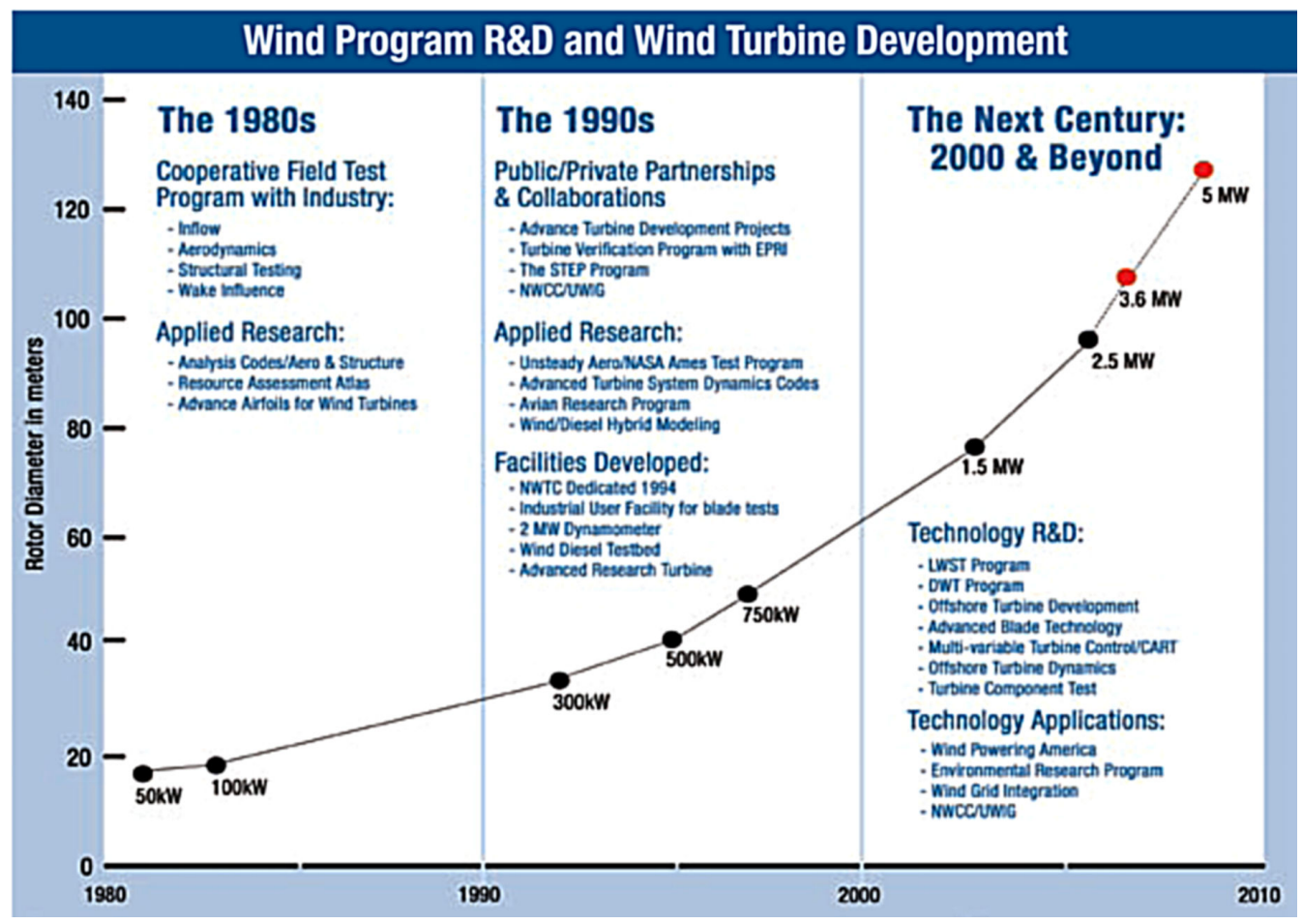

Figure 1.1. The wind-turbine upscaling phenomenon, [taken from DOE report 1] 


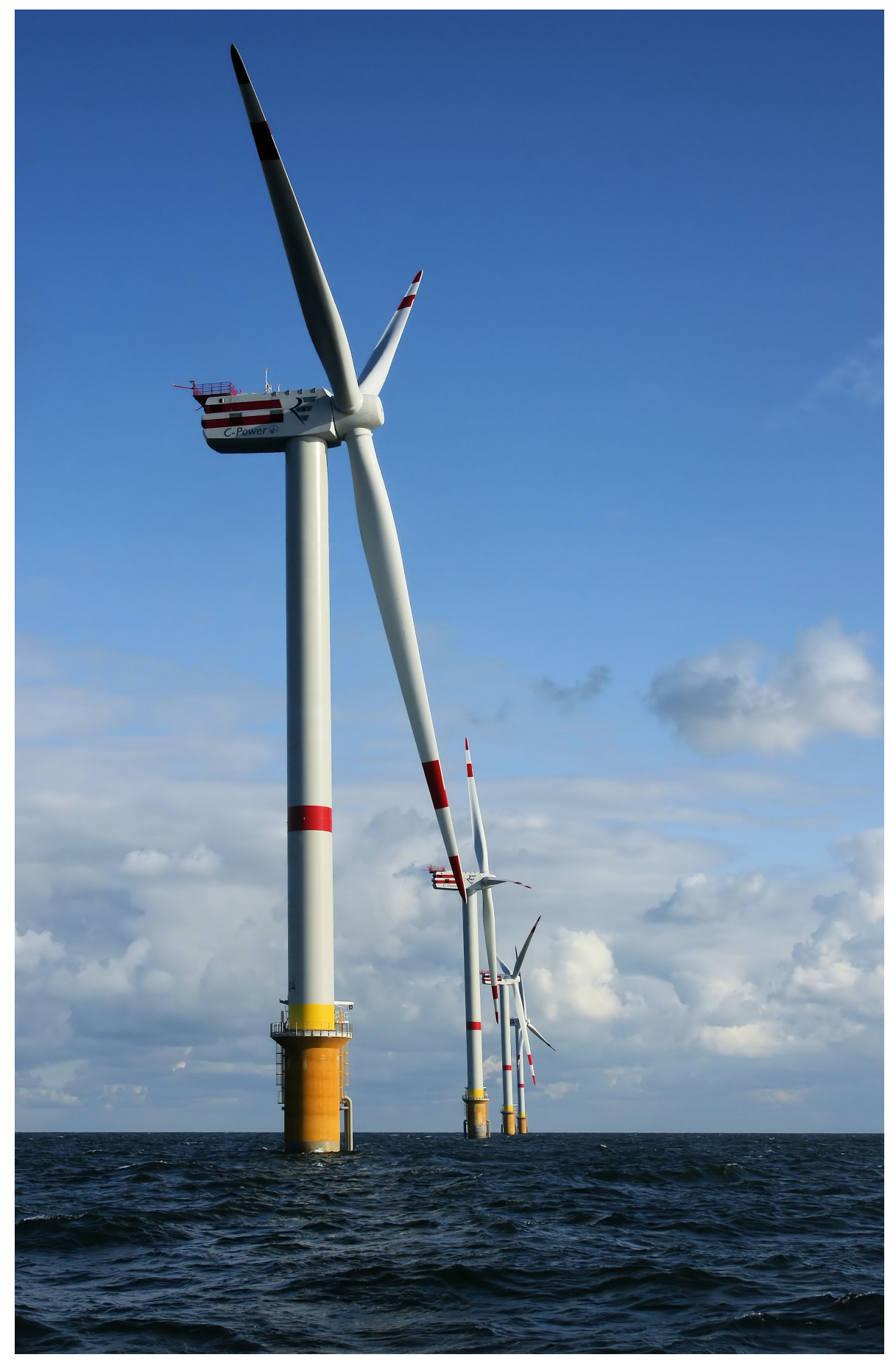

Figure 1.2. The REpower M5 5-megawatt turbine, with a rotor diameter of approximately 126 meters, [taken from Wikimedia Commons 20]. 
related to the aforementioned next generation of feasible upscaled turbines of cheaper construction, transport, and deployment that may further reduce generation costs at utility scale in both inland and offshore locations. Other challenges involve finding practical and economical ways of harvesting wind energy at the small-scale level for isolated consumers or for distributed-generation systems [24].

\subsubsection{The particular problem of floating foundations}

Current bottom-fixed technology is limited to water depths up to $50 \mathrm{~m}$. In deeper waters, support structures need to be more sophisticated. Long-term survivability of floating structures over many decades in the offshore oil industries and marine power inspired floating support designs for giant off-shore wind turbines.

A floating structure must have the capability to support the wind turbine in a harsh off-shore environment to support the weight with enough buoyancy; and to restrain heave motions such as pitch and roll and keep them within tolerable limits. Under the unpredictable environmental interference at sea, the platform dynamics may affect the reliability of the turbine's mechanical components, and complicate wake dynamics behind the rotor, which are even worse if considered in a off-shore wind farm. Thus, changes in design philosophy of a floating support to get a higher-standard compliant machine are required. Cost of such platform are likely to dominate the cost tradeoffs [2] in terms of computational modeling, field tests, manufacturing, and transportation to the site as for in-land wind turbines.

Hence, it is likely that the economics of deep-water wind turbines primarily depend on the extra cost of the floating foundations and the power distribution system. These additional costs are likely to be recouped by the increased energy production from high-speed offshore winds. There would also be an additional benefit in greater public acceptance of wind power due to lower environmental and visual impact. Butterfield et al. [2] reported some technical features that an optimum floating platform would embody by comparing several available platform designs. They also studied the issues and limitations which would challenge and direct future designs of wind-turbine floating platforms. A prototype of a floating platform will first be analysed for static stability, and the final design may be determined by some other critical factors [see 2, Table 1].

Current floating offshore wind foundations are not yet at the commercial stage and have a brief operational track record. Nevertheless, a preliminary feasibility analysis on the economics becomes possible once we establish the platform topology. In 2004, an MIT researcher, Paul D. Sclavounos, and his colleagues from NREL conducted a design called the Tension Leg Platform in which they integrated a wind turbine with a floating structure [25]. Several existing prototypes for offshore wind floating foundations, including those 


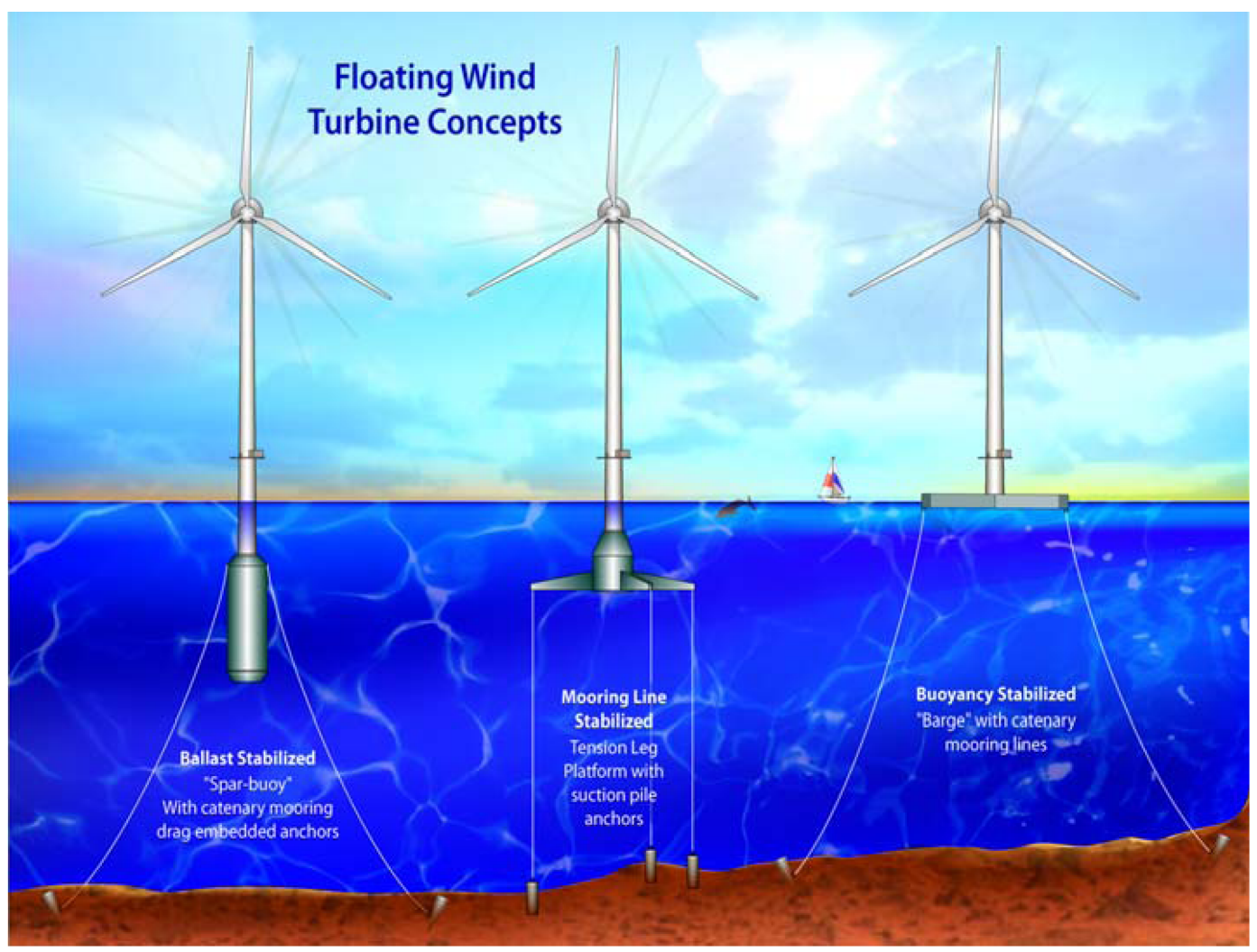

Figure 1.3. Alternative concepts for floating foundations for deep-water offshore Wind Turbines, [taken from NREL report 2].

considered by MIT, NREL, and DOE, are classified into three general categories [2]:

1. Ballast Stabilized: Use ballast weights which are hung underneath a central buoyancy tank to achieve stability, whereby a righting moment and high inertial resistance are provided to restrain the pitch and roll and usually enough draft is provided to offset heave motion. The left picture in Fig. 1.3 shows an example of this type of floating platform called the Spar-buoy [26].

2. Mooring-Line Stabilized: Use mooring-line tension to achieve stability, the Tension Leg Platform (shown in the center of Fig. 1.3) belongs in this category [26].

3. Buoyancy Stabilized: Use distributed buoyancy to achieve stability by the righting moment from the weighted water plane area [27]. A barge using this principle is shown on right in Fig. 1.3.

Other examples of floating foundations such as the semi-submersible TLP, the Dutch Trifloater, and the MIT Double Taut Leg Buoy are described in [2], together with details 
about the stability analysis and technical challenges on floating-foundation design. Some critical aspects of design, and the feasibility of multiple floating turbines, are discussed in Henderson and Vugts [28].

\subsubsection{Wind-farm array efficiency}

The idea of a wind farm is to locally concentrate wind turbines for electrical and commercial purpose. The multiple turbines introduced in a wind farm are intended to increase the total wind energy produced and lower the costs in repair and maintenance equipment and labor. However, a great amount of technical issues are brought up involving the placement of the turbines. One of the most significant problems is to improve the wind-farm array efficiency (i.e. the ratio between the collective power generated by the wind mill and the summation of the power that those same turbines would generate if they operated in isolation), that is, how to determine the locations of the wind turbines in an efficient way [29].

As we know, due to the terrain aspect, the wind resource may vary over a wind farm. Additionally, the wind power extraction by the upwind wind turbines causes lower wind speeds and introduces turbulent wake some distance downstream of the rotors. If wind turbines were closely spaced, this interference would affect one another, reducing the power output of the downwind turbines.

Extensive studies, both theoretical and experimental, indicate that interference due to the interaction of neighbouring turbines increases rather rapidly when they are spaced within $10 D_{r}$ ( $D_{r}$ is the rotor diameter). The number of turbines in the field is an important influence element on the array efficiency [30]. For instance, if we place an infinite number of wind turbines with $10 D_{r}$ apart, the array efficiency is limited to 60 percent. However, for the case of a finite number of turbines, the array efficiency is much higher. Johansson and Burnham [30] also shows the approximate efficiency of square arrays of array size from $2 \times 2$ to $10 \times 10$ : for a specified array size, the wind-farm array efficiency grows substantially as the turbine spacing increases from $4 D_{r}$ to $9 D_{r}$; for a designed turbine spacing, the wind-farm array efficiency drops rapidly as the array size grows.

Vortex dynamics can affect the wind-farm array efficiency and increase wake-induced fatigue. The secondary-wake patterns may be produced by the merging of vortices initially the size of the blade chord, and evolve into structures the size of the turbine itself. An picture taken by Christian Steiness, at the Horns Rev 1 offshore wind farm in the North Sea 14 kilometers west of Denmark, showed a good example of the turbulence wake effects behind offshore wind turbines. This wake effects resulted from unique meteorological conditions on 12 February 2008 at 1300 hours which created condensation of the humid air to make it 
visible behind the wind turbines [3].

The increase in size gets particularly intense when vortex coalescence occurs at highfrequency, low-amplitude vibration of the shedding body [9]. This secondary wake transition phenomenon is critical in wind farm spacing design. For instance, the hyper-Kármán vortex-street described in Chapter 2, section 2.2 could affect wind-farm array efficiency in such a way that its bigger vortices (wider-spaced wake) survive longer in the field and have a better chance of being advected onto the next row of turbines downstream. Thus, it is important to study the physics of wake dynamics behind bluff bodies in wind application .

\subsubsection{Ocean energy and hydrokinetic turbines}

Most of the challenges mentioned above for wind-turbine applications are shared by another emergent renewable-energy field. Like wind turbines, ocean energy technologies also promise attractive non-polluting alternatives to reduce the current dependence on fossil and nuclear-fuelled power plants to cope with the growing demand of electrical energy.

Ocean energy may be exploited in several ways such as Marine-current energy, Ocean thermal energy conversion, Wave energy, and Tidal barrages [31]. In the short term, marinecurrent energy and wave energy may be the most promising. The marine-current energy, in particular, inherits a substantial benefit from the vast experience in the research and development of wind turbines gained during the last decades in what, nowadays, is increasingly known as hydrokinetic energy conversion. Both wave energy and marine current energy convert the kinetic energy of moving water to power, without the impoundment or diversion of conventional hydroelectric facilities based on dams or penstocks. and therefore fall in the category of hydrokinetic energy conversion. Tidal barrages are similar to conventional hydroelectric dams on land and use the potential energy from height differences. OTEC is based on ocean water temperature differences and comprises thermodynamic devices for power production, as in thermal power plants. Osmotic pressure differences, as the name suggests, is based on a different principle than those mentioned earlier.

The whole hydrokinetic energy conversion system consists of four subsystems: the Hydrokinetic Conversion Device (HCD) itself; the support structure; the electric power converter and transmission system; and the remote communication and control link [31]. Conceptually, HCDs work in a similar way as wind energy conversion devices. In ocean-energy deployment, hydrokinetic turbines can be flexibly arranged so that the energy extraction from tidal and marine currents would basically be the same as a wind farm operates. Annual rated-power per square meter of rotor swept area of an HCD system at 2-3 m/s in water may be four times energy contrast to a similarly rated-power wind turbine [32]. However, the exploitation of this highly predictable energy source may be very costly. 
Many different HCD technologies exist today at various levels of development, as shown in 2006 data from the Carbon Trust for HCDs and wave-energy converters [4] It clearly reflects the long-term process required to turn a proof-of-concept idea into a full-scale prototype, and how many of the preliminary concepts do not survive the intermediate states of development.

First-generation devices that use conventional engineering components would be the extension of the present prototypes. These early devices can reach a depth of 20 to 30 meters and the rated-power might be in the range of 200 to $700 \mathrm{kWh}$ [5]. After further RD\&D, to reach economical viability, increase operational lifetime, and minimize maintenance, the second generation devices are expected within the next 10 years. These more advanced devices introduce specialized components, and can be installed at sites of depths more than 40 meters, where the tidal current potential is more abundant [5].

Currently, there are several HCD technologies under development, especially for MCP applications [33,34]. As in wind-energy conversion, turbines are considered the system of choice. However, some non-turbine systems have been proposed (mostly at the proof-ofconcept stage) and may become the innovative in this new technological field [35]. In spite of this keen interest in novel concepts, the primary types of HCDs are still the two classical categories of rotating machinery: (a) Horizontal-Axis turbines, similar to a modern wind turbine sniping a rotor perpendicular to the hub (axis), and (b) Cross-flow turbines, whose axis could be oriented either horizontally or vertically. Wind energy developers are quite familiar to these two types of HCDs. Besides, other turbine-based HCD systems are categorized by Khan et al. [35] into 1) Gravitational-Vortex systems; 2) Cross-Flow turbines, and 3)Venturi systems. Lago et al. [31] provides a summary of different categories of HCDs and discusses the advances and trends in the field.

Unducted horizontal-axis turbines have been the first HCDs deployed in bottom-fixed installations, followed by floating systems employing both vertical-and horizontal-axis turbines. The actual tendency indicates that the first type of system is going to be preferred in the short-term future for shallow water applications; floating systems are likely to be the main type in the so-called second generation of deep water HCDs. Non-turbine systems like the vortex-induced vibration, venturi, flutter vane, and fan belt, even though some of them already tested at the prototype level, are likely to achieve the commercial stage after the more classical turbine-based systems due to the innovative nature of their design.

The main challenge is not only extracting more energy per unit of rotor swept area, but doing it in a more economic and environment-friendly way. That is, energy-conversion efficiency alone is not as important as the overall cost-benefit relation of the HCD system operation. Hence, as in wind turbine application, to model an effective computational scheme for not only the HCD itself, but also others subsystems in whole hydrokinetic 
energy conversion system, is the way to go.

Current wind-turbine(hydrakinetic-turbine) blade technology based on composite laminates is labor-intensive and requires a highly-qualified workforce, creating a critical bottleneck in terms of industrial workforce and infrastructure that hampers a rapid expansion of wind-energy and ocean energy. It also poses a barrier to turbine upscaling by increasing the cost of the rotor as turbine size increases.

The structural conception of today's blades also poses huge challenges in terms of transport logistics and crane capacity. Transportation cost increases as blades grow in length. The risk of damage during transportation, and hence, the cost of insurance, also increases with length. Moreover, while the rest of the turbine subsystems may be treated as modules assembled on site, blades are one-piece monolithic components, substantially complicating transport logistics. Limitation in crane capacity is the other critical factor to take into account during the turbine assembly phase. Thus, transport and lifting logistics may impose a premature limit for turbine upscaling, even before the actual limits in blade length for the current manufacturing technology are reached. Blades operate under a complex combination of fluctuating loads, and huge size differences complicate extrapolation of experimental data from the wind-tunnel to the prototype scale.

Even if catastrophic resonance is avoided by careful design of the blade-structure natural frequencies, moderate (but undesired) flapping and twisting would certainly affect the energy-conversion efficiency of the rotor. Prolonged exposure to vibration would also compromise the blade's operational life through fatigue. The sort of complex wakes mentioned in previous sections have been observed at a wide range of scales, if they occur in the wake of a blade, load peaks due to their irregularity may compromise its structural integrity.

From the scientific point of view, it is even more challenging to cope with the case of a blade than the case of flow passing a cylinder (or any other bluff bodies) because, depending on the angle of attack, the airfoil profile of the blade section may behave as a streamlined body or as a bluff body. The picture is even more complex when operational factors, like variation of the angle of attack in pitch-controlled machines are taken into account. In stall-controlled machines, the inner part of the blade (which is in stall condition) actually behaves as a bluff body, while the rest behaves as a streamlined one. The suitability of the KLE method for accurate simulation of complex wakes would give our aeroelastic model the capacity to capture these features.

Hence, computer models of fluid-structure interaction phenomena are particularly relevant to the design and optimization of wind-turbines and hydrakinetic turbines. The windturbine and hydrakinetic-turbine industry is increasingly using computer models for blade structural design and for the optimization of its aerodynamics. But the complex interaction 
of physical processes that characterize the coupled aeroelastic problem still exceeds the capacities of existing commercial simulation codes. The result is an industry that is cautious with the introduction of new concepts in order to ensure reliability. Innovations are likely to introduce changes in structural response and may possibly require different control strategies, which should be taken into account if the development of a new prototype blade is considered. Research efforts within the established parameters of the compositelaminate monolithic blade concept would not produce the breakthrough that is needed in wind-power evolution. A better understanding of the underlying physics is needed in order to introduce innovative concepts like modular blades and improved control strategies. This is where the KLE plays a pivotal role owing to its ability to create a common framework for modular integration of the aeroelastic model with the control system dynamics.

\subsection{Dissertation goals}

The aeroelastic dynamics in slender bodies depends not only on the characteristic modes of the body structure itself, but also on the frequency and amplitude of the fluctuating aerodynamic forces. Vortex-induced vibration can lead to catastrophic failure of engineering systems. A resulting periodic vibration essentially depends on the work done by the fluid on the body over a cycle. This is associated with the timing of the vortex dynamics because the induced side force caused by the body motion significantly influences the net energy transfer [13]. A significant challenge in analyzing the aeroelastic problem in these systems such as wind turbine blades, air-plane rotors, is the fact that a vortex wake different from the classic Karman vortex street can be produced by an oscillating body, which would translate into a complex fluctuating aerodynamic force.

The focus of the current dissertation is to study qualitatively the underlying physics of vortex-shedding and wake dynamics in long aspect-ratio aerodynamics in incompressible viscous flow through the use of the KLE method. We carried out a long series of numerical experiments in the cases of flow around the cylinder at low Reynolds numbers. We verified the wake length of the closed near-wake behind the cylinder and analysed the decay of the wake at the wake formation region, and then studied the St-Re relationship at the Reynolds numbers right before the wake sheds compared to the experimental data. Later, a theoretical model of the decay of the wake and a low-dimensional model for the fluid physics are proposed. The study of flow at low Reynolds numbers provides an insight in the fluid physics and also plays a critical role when applying to stalled turbine rotors. In the long run, it aims to provide a better understanding of the complex multiphysics problems involving fluid-structure-control interaction through improved mathematical computational models

of the multi-process. Besides the scientific conclusions produced, the research work on streamlined and bluff-body condition will also serve as a valuable guide for the future de- 
sign of blade aerodynamics and the placement of wind turbines and hydrakinetic turbines, increasing the efficiency in the use of expensive workforce, supplies, and infrastructure.

\subsection{Dissertation outline}

Chapter 2 presents the theoretical basics of vortex-shedding and wake dynamics behind bluff bodies, including the Kármán vortex street and wakes behind oscillating cylinders. In sction 2.1, we present "The study of the velocity field using Helmholtz decomposition and the evolution of eddy structures" and "vorticity profiles and decay law of the eddy structures in the vortex street"; In section 2.2, we present three interesting aspects of the flow behind oscillating bodies: 1) On the boundaries between vortex synchronization regions in the WR map; 2) Formation of $\mathrm{P}+\mathrm{S}$ vortex streets at low Reynolds number; 3) The formation of a hyper-Kármán vortex-street. These are out of the numerical implementation of the KLE method mentioned in chapter 4 and motivate the numerical experimentation on the wake decaying law at the wake formation region around a cylinder at low Reynolds numbers, which will be discussed in chapter 5 .

In chapter 3 we present the mathematical basis of the solution of the incompressible viscous flow and the theoretical basis of the KLE method. The first section 3.1 gives an introduction to the incompressible Navier-Stokes equations. The second section deals with some of the more popular formulations of the Navier-Stokes equations modeling a viscous incompressible flow of a homogeneous fluid in an inertial frame of reference, along with some discussion on the issues of boundary conditions. To find a numerical solution to the NavierStokes equations would be to decide upon the set of variables representing the equations followed by a group of boundary conditions. The two main formulations of the NavierStokes equations are of the primitive variable formulations and the non-primitive variable formulations. Both have their own set of advantages and disadvantages, which shall be discussed in brief, but in both cases the major problem and the oldest point of contention are the boundary conditions.

Chapter 4 is concerned with the hybrid methods based on a Vorticity-Velocity approach to solve the Navier-Stokes equations numerically and a robust mathematical computational method: the KLE method. It starts with the introduction of the hybrid-method family: the vorticity-velocity formulation of 2-D and of 3-D. The next section goes on to introduce the KLE along with its variational formulation. The KLE method was first introduced by F.L.Ponta in a paper [36]. It is a Vorticity-Velocity method which decouples the evolution of vorticity from a velocity field from the spatial solution. Vorticity is advanced in time by integrating a vorticity transport equation for which an initial velocity field is obtained by solving the weak form of the KLE. The KLE in turn is solved using the vorticity field 
obtained by integrating of vorticity from the previous time step in time. The no-slip, nonormal flow Boundary conditions for velocity required for solving the KLE are solved over a sequence of two steps. This basically involves two integral projection in each time step ensuring compatibility of the two fields at each step. Then it deals with the numerical implementation of the KLE at the end.

In chapter 5 we present the analysis of the numerical experimentation results for the oscillatory regime right before the onset of vortex shedding for circular cylinders. We found a theoretical model that describes the time evolution of the amplitude of fluctuations in the vorticity field on the twin vortex wake, which accurately matches the numerical results in terms of the frequency of the oscillation and rate of decay. We also proposed a model based on an analog circuit that is able to interpret the concerning flow by reducing the number of degrees of freedom. It follows the idea of the non-linear oscillator and resembles the dynamics mechanism of the closed near-wake with a common configured sine wave oscillator. This low-dimensional circuital model may also help to understand the underlying physical mechanisms, related to vorticity transport, that give origin to those oscillations.

Finally, chapter 6 presents the conclusions for this dissertation work as well as the outlook for further work based on the analysis of the material presented in the previous chapters. 


\section{VORTEX-SHEDDING AND WAKE DYNAMICS BEHIND BLUFF BODIES}

Wakes generated by bluff-bodies, particularly wakes behind cylinders, have been studied by many researchers because they not only help to understand the underlying nature of turbulent flows, providing insights into a widespread physical topic which makes appearances in many scientific disciplines, but also in a variety of engineering applications. In a case like a rotor blade where a body is subjected to a complex motion due to the intrinsic operation of a certain mechanism and external influence, as mentioned in chapter 1, section 1.2.3, the challenge to study non-linear dynamics is still greater.

Other than the famous Kármán vortex street as seen in the case of uniform flow around a stationary cylinder, oscillating cylinders produce some more complex patterns in the vortex wakes. The dynamics of the vortex-wake shed from the body results in fluctuating aerodynamic forces being exerted on the body itself. The frequency and amplitude of these forces affect the aeroelastic dynamics together with the characteristic modes of the body structure. For example, Figure 2.17 shows a non-symmetric periodic wake behind an oscillating cylinder with three vortices per cycle, and Figure 2.20 shows a non-synchronous wake with a complex pattern of vortical structures being shed. These numerical results from Ponta and Aref [10], computed with the KLE method, show favorable agreement with Williamson and Roshko's experimental observations [9]. In both cases the net impulse on the wake in each cycle is zero, but the distributions of the instantaneous forces along the cycle are far from regular.

\subsection{The Kármán vortex street: a classic case-study}

The famous theory on Kármán-Bénard vortex street was first published by Kármán in 1911, although Prof. Bénard claimed priority for earlier experimental observation of the same fluid phenomenon in 1908. A thorough and significant series of studies have since been completed. A substantial portion of these investigations have been focused on experimental 
observation. Recently, however a lot more theoretical/numerical studies have been done. Despite this, a single model that can clearly explain the physical mechanism of how a vortex-street wake is formed has yet to be developed.

A classic case of study is the formation and development of the Kármán vortex street behind a translating cylinder. The circular cylinder is known for the most universal geometry in engineering applications, appearing in chemical, civil, mechanical, nuclear, and offshore engineering. More importantly, the flow around a circular cylinder shares most of its characteristic features with the flow passing through many other types of bodies. Despite the shape differences and the presence/absence of different sharp edges, all bluff bodies have some similar characteristics in the disturbed flow regions around them. Even the flow around airfoils and flat plates at high angles of incidence appear similar to flows around bluff bodies. Both flows have a common characteristic known as eddy streets, that is, similar vortex structures developed in the separated region behind bluff bodies [37]. Thus, the research on flow around a bluff body such as a circular cylinder allows an opportunity to generalize a physical phenomenon that can then be applied to many disciplines. Usually, an array of alternating well-defined vortices can be found behind a circular cylinder at a certain range of Reynolds numbers within the laminar regime. Depending on Reynolds numbers, a Kármán vortex street produced in a steady stream behind a bluff-body sheds periodically with different shedding frequencies. Studying the shedding frequency of the wake and its affect on the bluff-body leads to a better understanding of the fluid dynamics in bluff-bodies, and especially helps to explore the research on structural-fluid interaction. Vortex-induced vibrations is a very important topic in the study of wake dynamics. It may cause resonance, which is a significant consequence when the shedding frequency of a vortex is close to that of the structure's vibration.

Applying the Kinematic Laplacian Equation (KLE) method, described in chapter 4, Ponta conducted numerical experiments to study the case of a circular cylinder in a fluid at rest, given a sudden velocity and then maintained at that velocity. One of the meshes of triquadrilateral elements used in his research is shown in Figure 2.1.

Ponta discussed some results at several Reynolds numbers which were computed numerically through the KLE method. Figure 2.2 illustrates the superimposition of vorticity isolines and the solenoidal component of the velocity field $\boldsymbol{v}_{\omega}$ at $R e=140$. Vorticity isolines plotted here were captured during one vortex-shedding cycle in steady flow around a circular cylinder. The velocity field is shown by arrows. The vorticity evolution due to the influence of the eddy-structure defined by the streamlines of $\boldsymbol{v}_{\omega}$ can be followed clockwise from the bottom-left panel to complete the cycle [6]. Figure 2.3 presents a close-up view of the last figure which shows the early stages in the process of vortex-formation. It shows how the shear layers roll-up around the eddy-structures in $\boldsymbol{v}_{\omega}$. 


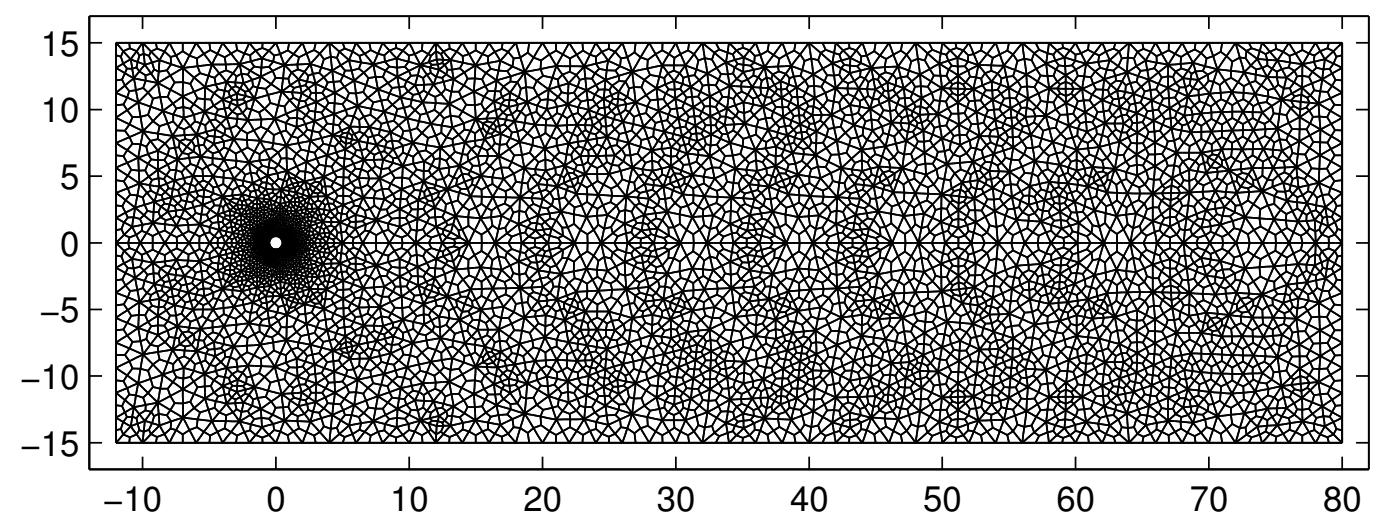

Figure 2.1. A Tri-quadrilateral finite element mesh used in a study by Ponta (lengths in diameters of the circular cylinder), [Figure created by author from data in 6].

For the case of $R e=100$, Ponta compared a experimental flow visualization of a Kármán vortex street behind a cylinder from M. M. Zdravkovich with the numerical vorticity field computed by the KLE method [6]. Both the flow visualization and the magnitude of vorticity shown in the experimental photo are somewhat different than the results from the numerical computation. Nevertheless, the shape of the vortices and more importantly the spacing correspondence gives confidence in the accuracy of the numerical simulations.

For $\operatorname{Re} 50<R e<180$, the measurement of the dominant frequency of vorticity fluctuations, $f$, had been taken at a group of points along the vortex street. At these points the Strouhal number, $S t=f D / U$, was then computed. For all the points considered, the dominant frequency was the same and clearly formed at the initial stages of wake formation. Unlike the frequency, the amplitude of the fluctuations displayed a transient state before stabilized some distance downstream. A $S t$ versus Re relationship was plotted in figure 2.4 and was confirmed with the experiments by Williamson [7].

\subsubsection{The study of the velocity field using Helmholtz decomposition and the evolution of eddy structures}

F. L. Ponta discussed two issues [39] on this subject. The first was about the vorticity evolution. The second was how to identify the eddy structures. To study what determines the vorticity distribution, Ponta used the KLE method to simulate the flow passing through a circular cylinder over $50<R e<180$. Then, the vorticity was calculated by applying the curl of the velocity field that was found using direct numerical simulations. However, it is more challenging to study how to identify the structures of the eddies. The choice of frame 

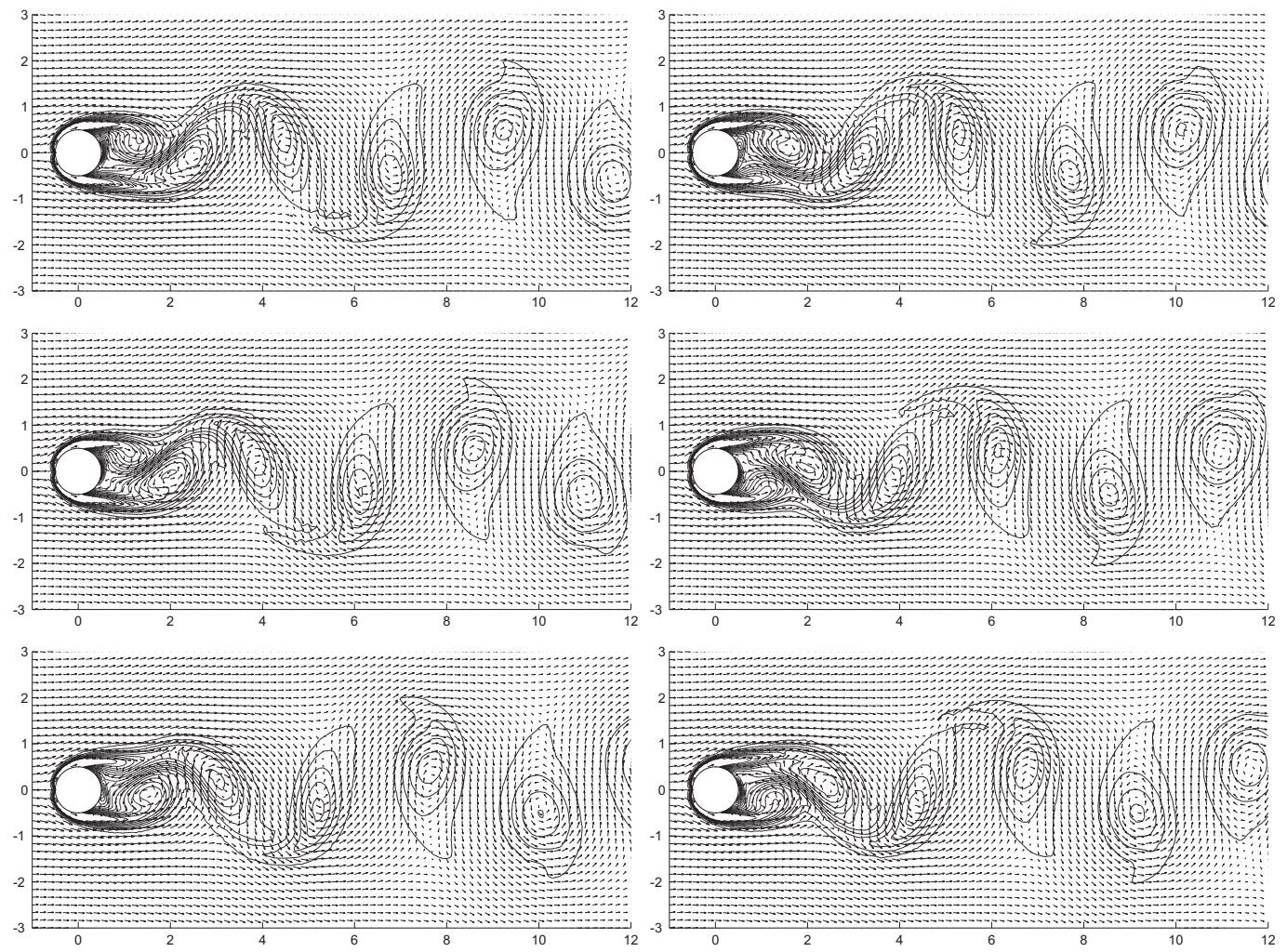

Figure 2.2. For $R e=140$, the rearrangement of the eddies during one vortex-shedding cycle in the wake behind a circular cylinder is shown using the vorticity isolines and the pattern of streamlines of $\boldsymbol{v}_{\omega}$ [Figure created by author from data in 6]. Sequence should be followed clockwise.

of reference strongly influences the topology of the velocity field [39]. Considering a towed cylinder in an initially stationary fluid, an observer travelling with the cylinder would observe an incipient eddy structures near the bodies surface and the far wake would display wavy streamlines without eddies. In comparison, if the observer were stationary, the normal eddy structure in a vortex street would be seen in the far wake and the vorticity near the cylinder would become distorted. Batchelor [40], Plate 11 shows a very good example of this. Thus, failing to choose a proper frame of reference would result in the conclusions concerning the formation mechanisms. Furthermore, when the reference frame moves with the cylinder, one may observe the eddies advecting downstream, initially at a low speed zone near the cylinder and then accelerating downstream to the far wake where a steady advection regime is achieved. To properly describe the streamline pattern, a desired frame of reference must track every eddy as it accelerates downstream. However, this would complicate the situation and result in strongly biased conclusions. Hence, the incompressible velocity field was decomposed using a frame of reference that moved with the cylinder. The velocity field can be written as follows: $\boldsymbol{v}=\boldsymbol{v}_{\omega}+\boldsymbol{v}_{h}$. Here, $\boldsymbol{v}_{\omega}$ is the solenoidal component of $\boldsymbol{v}$ and has the same curl as $\boldsymbol{v}$, while $\boldsymbol{v}_{h}$ is the harmonic component. The 

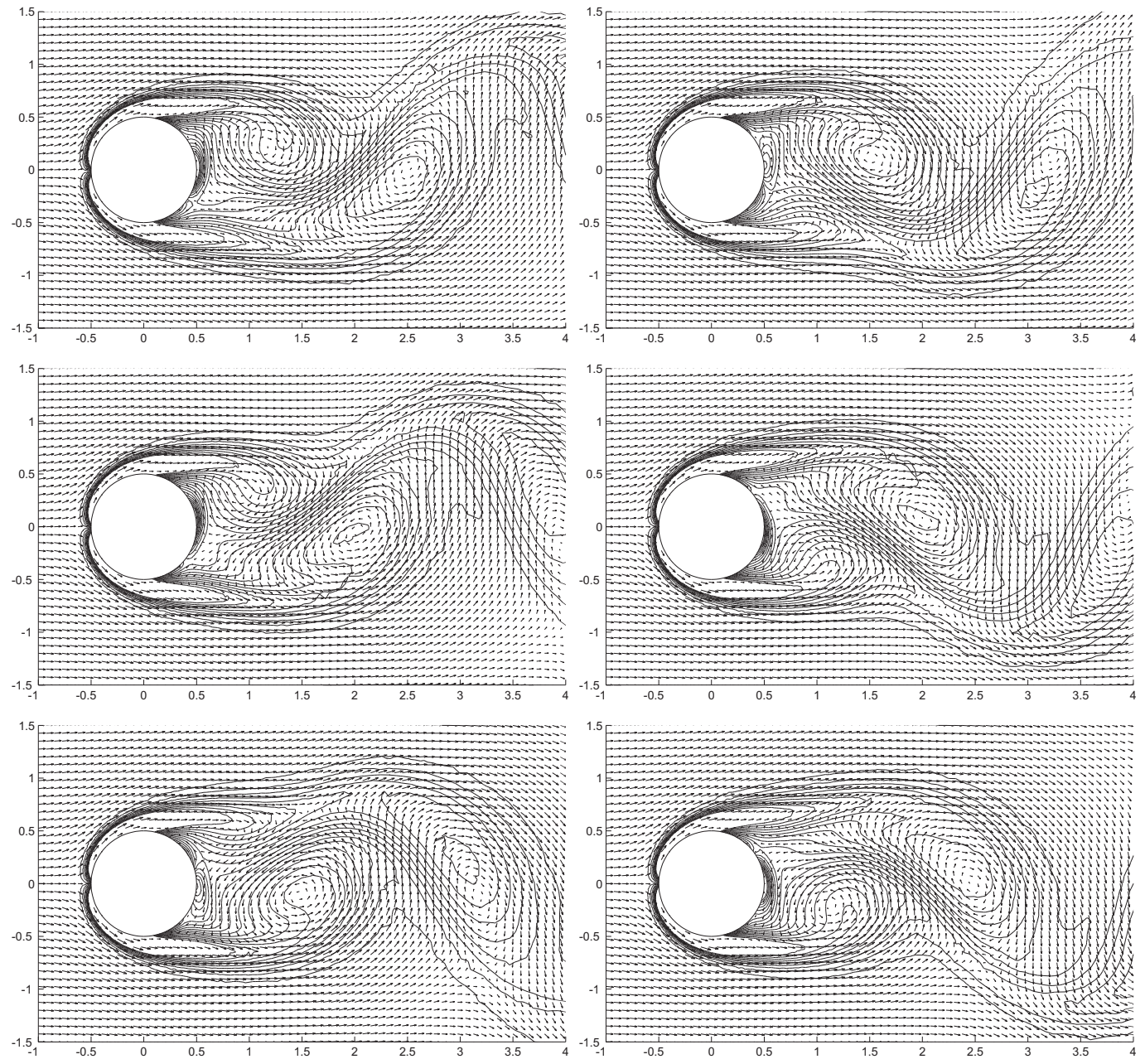

Figure 2.3. For $R e=140$, the vortex-formation during one vortex-shedding cycle in the wake behind a circular cylinder is shown using vorticity isolines and the pattern of streamlines of $\boldsymbol{v}_{\omega}$, [Figure created by author from data in 6 ].

two-dimensional vorticity transport equation, Eq 2.1, can be rewritten as in Eq 2.2. This is due to $\boldsymbol{v}_{h}$, the harmonic component, having had no contribution to the diffusion of vorticity and only influenced the advection process:

$$
\begin{gathered}
\frac{\partial \boldsymbol{\omega}}{\partial t}=-\boldsymbol{v} \cdot \boldsymbol{\nabla} \boldsymbol{\omega}+\nu \nabla^{2} \boldsymbol{\omega} \\
\frac{\partial\left(\boldsymbol{\nabla} \times \boldsymbol{v}_{\omega}\right)}{\partial t}=-\left(\boldsymbol{v}_{\omega}+\boldsymbol{v}_{h}\right) \cdot \boldsymbol{\nabla}\left(\boldsymbol{\nabla} \times \boldsymbol{v}_{\omega}\right)+\nu \nabla^{2}\left(\boldsymbol{\nabla} \times \boldsymbol{v}_{\omega}\right) .
\end{gathered}
$$




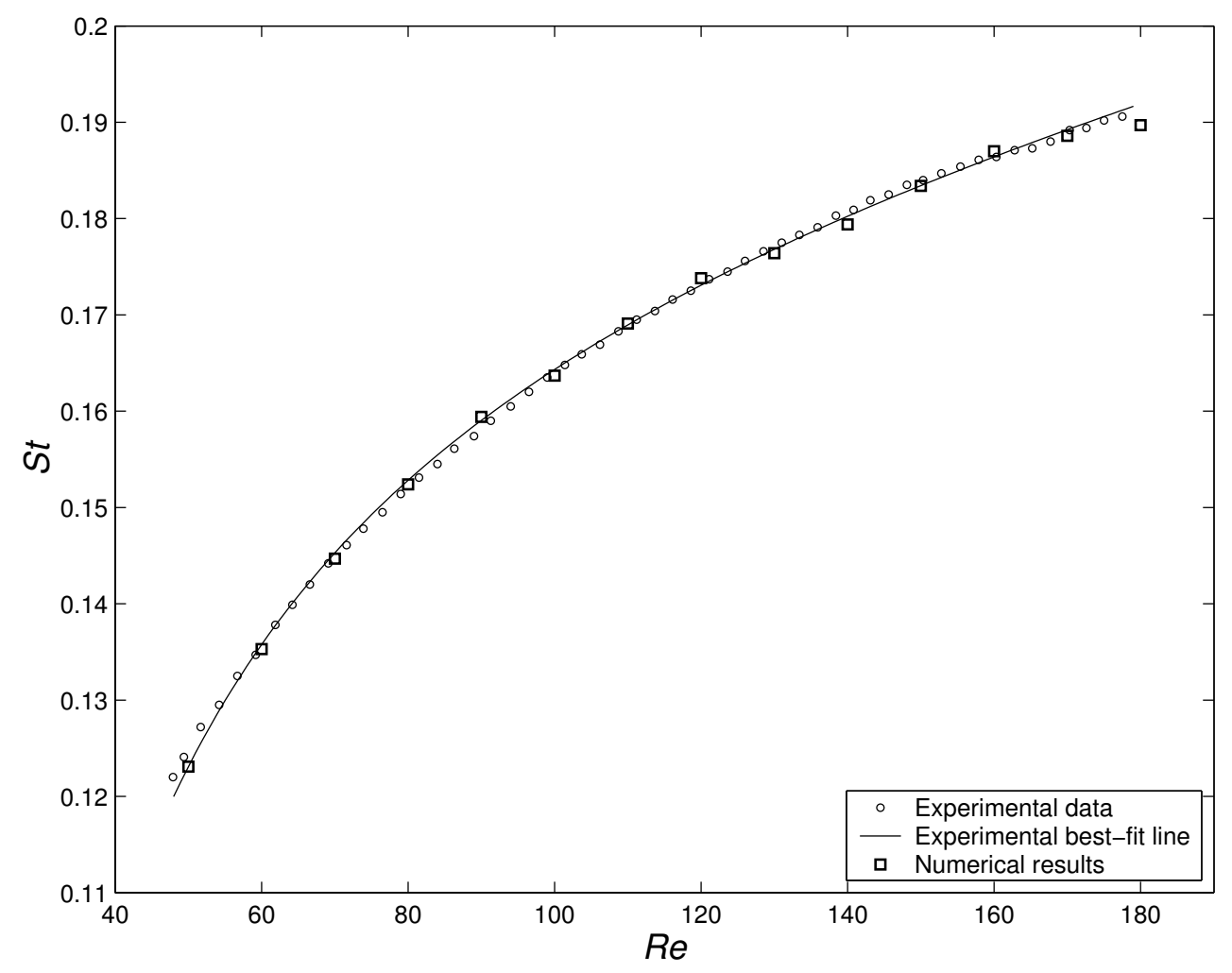

Figure 2.4. Comparison of the numerical Strouhal number using the KLE method with Williamson's experimental measurements [7] for $50<R e<180$.

The streamline pattern in $\boldsymbol{v}_{\omega}$ defines the eddy structures and $\boldsymbol{v}_{h}$ is assigned to advect these eddy structures.

In Batchelor [40], Section 2.7, it is pointed out that, for prescribed velocity conditions, $\boldsymbol{v}_{h}$ can be uniquely determined on the boundary of the computational domain. In the numerical simulations performed with the frame of reference having moved with the cylinder, Ponta completed the evaluation right before any vorticity reaching the external boundary of the working domain. Hence, the velocity on the solid surface was set to be zero and a stream of uniform velocity was applied on the external boundaries. $\boldsymbol{v}_{h}$ and $\boldsymbol{v}_{\omega}=\boldsymbol{v}-\boldsymbol{v}_{h}$ were both determined uniquely. By analysing the streamline pattern in $\boldsymbol{v}_{\omega}$ to study flow topology, the issue of selecting a frame of reference can be circumvented.

The top and bottom image of Figure 2.5 show the superimposition of the instantaneous velocity arrows of $\boldsymbol{v}$ and the superimposition of $\boldsymbol{v}_{\omega}$ on the vorticity isolines in the far wake respectively. As shown, the arrows of the velocity field $\boldsymbol{v}_{\omega}$ are tangent to the isolines of vorticity inside the eddy cores. That is, distribution of the vorticity does not change relative to $\boldsymbol{v}_{\omega}$. This means, $\boldsymbol{v}_{h}$ causes the advection of the full vortex structures downstream, while 
$\boldsymbol{v}_{\omega}$ causes the advection of eddy structure vorticity internally. This helps to rearrange the vorticity inside the vortex core [39]. Figure 2.5 also illustrates how the pockets of vorticity advect beyond the confluence point and form the tails. Here, a confluence point is seen in the streamlines of $\boldsymbol{v}_{\omega}$ as a saddle point. The vortex cores, in the far wake, simply decays due to a steady viscous effect as advected by $\boldsymbol{v}_{h}$ downstream. The preceding explanation shows a good consistency with observations in the experiments and confirms $\boldsymbol{v}_{h}$ is the cause of the advection in the far wake.
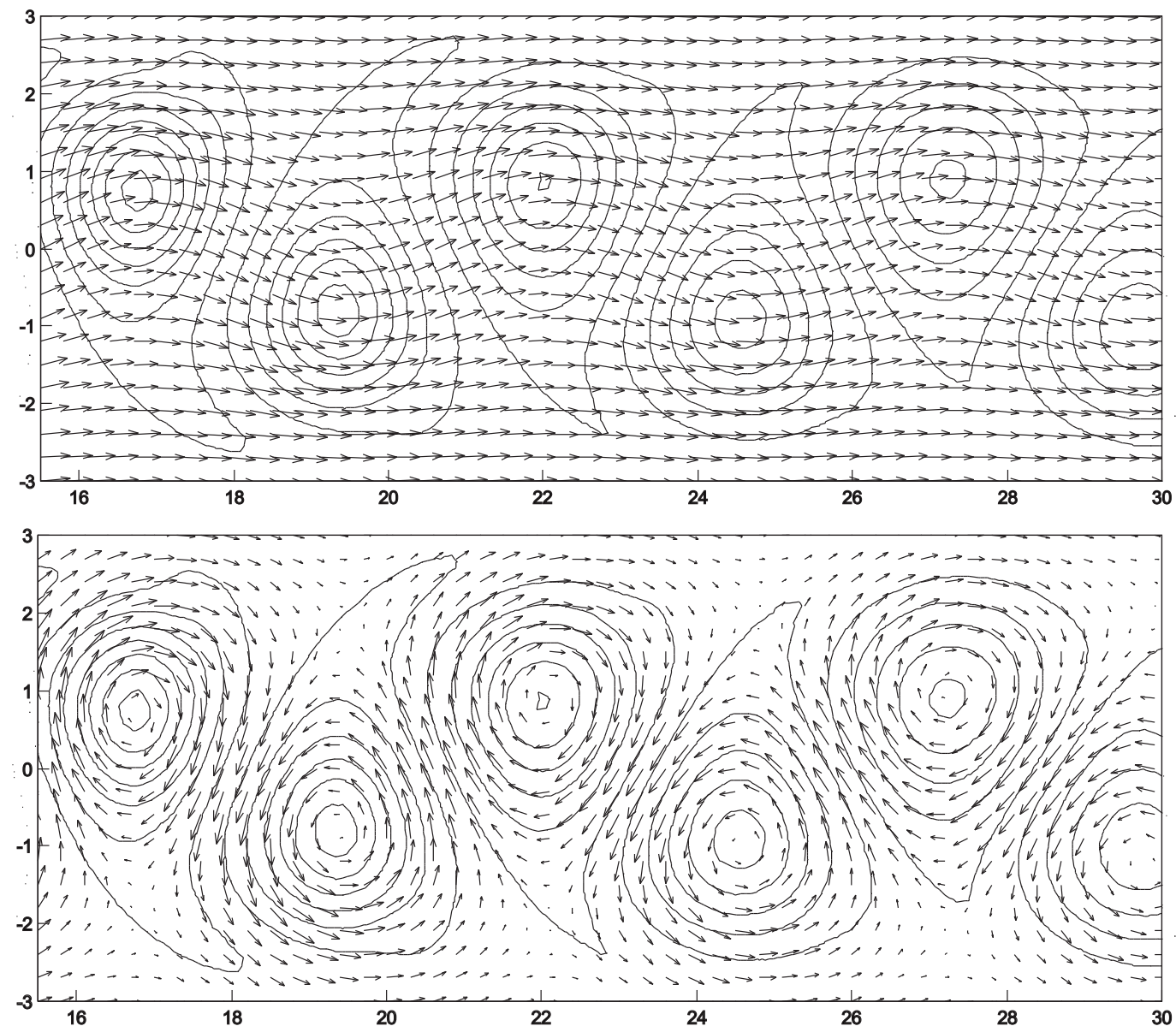

Figure 2.5. For $\mathrm{Re}=100$, upper panel shows the superimposition of vorticity isolines with pattern of streamlines of $\boldsymbol{v}$, while the lower panel presents the superimposition of vorticity isolines $\boldsymbol{v}_{\omega}$ [Figure created by author from data in 8$]$.

Also, Ponta argued that $\boldsymbol{v}_{h}$ determines the advection in the near wake. To verify this, Figure 2.3 shows the superimposition of $\boldsymbol{v}_{\omega}$ streamlines and vorticity isolines with a sequence of plots of the formation of vortex structures in the near wake. As shown, the rolling-up of the shear layer of positive vorticity around the incipient eddy starts on the lower right of the cylinder. This process will continue until vorticity all around the periphery of the initial eddies reaches a roughly homogeneous distribution. It is noted that the vortex core 
has been formed while the outside of the eddy is still in the process of its development, dissipating its vorticity into the surroundings.

Figure 2.2 shows the two main processes in the formation of the vortex street [39]. The process of stretching of the eddy structures which was produced by the interactions of neighbour vortices took place first in the shedding of the eddy structures. This results in an $\mathrm{S}$ shape for the associated vorticity block which has been documented in multiple experimental flow-visualization and computational simulations. The eddy was initially connected to the separated shear layers that created it by having had rolled-up, but during the rearrangement process, this connection was severed. Then, three nearly simultaneous processes took place caused by the local advection due to $\boldsymbol{v}_{\omega}$ combined with the forces of viscous diffusion[39]: 1) the severed vorticity traces advected into the core of the vortex and united with the vorticity of the core; 2) a new tail was formed by the traces of vorticity which had fallen past the confluence point from the vortex core, which was visible in the early stages of the evolution of the vortex street, as seen in Figure 2.5: 3) inside the vortex core with vorticity isolines tangent to the arrows of the $\boldsymbol{v}_{\omega}$ velocity, vorticity got homogenized along the periphery.

When the rearrangement of the eddy structures was completed, the vortex street would be fully formed. From this point on, the eddies decay under the steady viscous effect as the vorticity was cancelled out by the interactions between eddy cores of opposing rotation.

\subsubsection{Vorticity profiles and decay law of the eddy structures in the vortex street}

The first vortex street structure theoretical model was derived by Kármán. This model considered an ideal flow with the stability of point vortices staggered in two parallel rows. For the arrangement to be stable, the space between the point vortices rows should be 0.281 times the streamwise separation between consecutive vortices in a given row. This ratio was determined analytically [41,42]. When this was examined using real fluid eddy streets by Bénard [43], it was found the rows of point vortices were further apart than analytically predicted and continued to move further apart down the wake. Zdravkovich [37] found the laminar eddy streets stability conditions varied relatively little in the spacing $a$ in given row compared with the variation between the rows. These tests imply the ratio calculated by Kármán is primarily a function of the space between the rows. The spacing between the vortices in the row appears to be strongly dependent on Re while the spacing between rows appears to be independent of Re (see Zdravkovich [37], Sec. 3.8, where data on this ratio from multiple sources is presented).

Improving on previous models Hooker [44] substituted the immutable point vortices with eddies that have a Gaussian distributed vorticity, $\omega$, which depends on the radial distance 
from the vorticity center. Diffusion effects can now be captured in the model and allow the eddies to expand as it moves down stream. At the initial condition the infinite street of vortices are modelled as point vortices that a subjected to viscous action. Immediately after the initial condition the infinite velocities and pressures of the singular points disappear and "the vortices commence to diffuse, thereby realizing a state of flow more in keeping with those actually prevailing in any observed street." Hooker's results match the process of diffusion and decay of the classical solution proposed by Lamb [45](Chap. XI, Art. 334a). In Lamb's solution, the vorticity spreads into the fluid as heat from an instantaneous linesource would diffuse into an infinite medium. This so called Lamb-vortex model finds the distributions of vorticity, $\omega$ and circumferential velocity, $v_{\theta}$. Both $\omega$ and $v_{\theta}$ have a functional relationship to the radial distance, $r$, and time, $t$

$$
\begin{gathered}
\omega=\frac{\Gamma}{4 \pi \nu t} e^{-r^{2} / 4 \nu t}, \\
v_{\theta}=\frac{\Gamma}{2 \pi r}\left(1-e^{-r^{2} / 4 \nu t}\right),
\end{gathered}
$$

where $\nu$ the kinematic viscosity and $\Gamma$ is the circulation of the original line vortex.

The overall vorticity enclosed within the radius $r$ is

$$
\Gamma_{r}=\int_{0}^{r} 2 \pi r \omega d r=\Gamma\left(1-e^{-r^{2} / 4 \nu t}\right)
$$

and it can be shown the vorticity within a large radius is approximately equal to $\Gamma$ for finite values of time. The vorticity is only diffused by viscous action throughout the fluid but not destroyed.

A maximum of $v_{\theta}$, using (2.4), will be given at a radius which satisfies

$$
\frac{r_{c}^{2}}{4 \nu t}=1.26, \quad \text { or } \quad r_{c}=2.245 \sqrt{\nu t}
$$

The age of a vortex is then indicated by the radius of maximum $v_{\theta}$. Alternatively, the peak value of $\omega\left(\omega_{p}=\frac{\Gamma}{4 \pi \nu t}\right)$ can also be taken as an indicator of age.

Hooker [44] defined the core radius at the point of maximum velocity. The effect of the viscous term on the fluid velocity is insignificant outside a given radius. For eddies in water where $t \leq 30 \mathrm{~s}$, this radius is about $2 \mathrm{~cm}$. Most of the vorticity will then remain inside this radius for the 30 seconds being considered. Experimentally, Hooker formed 
a vortex street with constant longitudinal spacing $a$ at $6.9 \mathrm{~cm}$. Using Kármán's stability condition ratio of vortex rows spaced 0.281 times further apart than the distance between consecutive vortices, would correlate to an initial point-vertex Kármán street with the rows $1.935 \mathrm{~cm}$ apart. At a point defined as $\mathrm{P}$, Hooker replaced the ideal point vortex with a viscous eddy determined by 2.4. The velocity field created by the point vortex at $\mathrm{P}$ was then replaced with a field corresponding to its viscous counterpart. According to Hooker "It was futile to replace any other vortex by the viscous form for each will be at a distance greater than $4 \mathrm{~cm}$ from $\mathrm{P}$ and the diffusion term is negligible under these circumstances. The velocity field of all the remaining diffuse vortices (excluding P) will be the same as if they were concentrated as filaments at their original positions". Because the velocity at point $\mathrm{P}$ due to vortex $P$ is zero, Hooker's velocity distribution results in the same convection speed for the complete vortex system due to mutual actions in the same way as Kármán's experiment. Therefore, the spacing of consecutive vortices in a given row is not effected by diffusion and the apparent center of the vortices move away from the wake axis as time increases. This apparent center does not coincide with the maximum vorticity but with the point of zero velocity. The maximum vorticity is found at the point of initial concentration in-line with each row of downstream vortices. According to Hooker, the points observed in experiments were the zero velocity points around which the fluid appeared to circulate.

Comparing Hooker's partially viscous theory to measurements, Timme [46] found a favourable agreement for transverse and streamwise velocity profiles. Later Schaefer and Eskinazi [47] made a similar comparison and confirmed the results. The downstream widening of the two rows of vortices predicted by Hooker [44] roughly matched experimental measurements for $t \leq 30 \mathrm{~s}$. A second widening, which was not predicted by Hooker was found some time after $\mathrm{t}=30$ seconds. Hooker noted several considerations that may explain the discrepancies between his theory and experiments:

(i) Real vortices are never filaments during the course of their existence.

(ii) In reality the vortices are of unequal 'age'.

(iii) Street lengths are finite in reality.

Points (ii) and (iii) "are secondary effects provided that we consider a region of the street at which the vortices have not spread sufficiently to intermingle" according to Hooker. Naturally the next question is what happens once the eddies are able to intermingle. To investigate this Green and Gerrard [48] pulled a cylinder along a tank at $\mathrm{Re}=80$ to produce a eddy-street wake. Using an interferometer to visualize dimples on the water's surface, it was found that the mutual interaction of adjacent dimples induced displacement away from the wake axis. The dimple's depth is reduced and the radius is increased by the diffusion of the eddies, which in turn is caused by viscous dissipation. 
The concept that the location of maximum velocity may define the viscous core border was reconsidered in a theoretical study by Zdravkovich [37, Sec. 11.14]. The Radius of influence would then extend to $r_{c}=4.3 \sqrt{\nu t}$. At the point $r_{c}$, the difference between the induced velocity due to a point vortex and a viscous vortex is within $1 \%$. The velocity distribution difference caused between a point vortex and a viscous vortex will introduce an imbalance by reducing the induced velocity when the adjacent vortices are within the viscous core of the observed vortex. Zdravkovich proposed a differential equation valid up to the point where a second core in the other row reaches the observed vortex. This equation would model the widening of the street as a function of this imbalance. According to [37], "The path of vortices becomes continuously modified as they proceed downstream. The strength of eddies is gradually reduced as the viscous cores overlap and the vortices of opposite sign cancel each other. The mechanism of diffused vortex core interaction is little understood at the present."

The vorticity distribution is a function of the eddy structure and the time evolution. After this the eddy is established in the vortex street [8]. A 3-D perspective view of a twelvevortex series vorticity distribution is seen in Figure 2.6 (a). Here, the Kármán-street wake for $\mathrm{Re}=100$ is shown with geometrical coordinates given in diameters of the cylinder, $\mathrm{D}$, centered at the origin $\left(x^{*}=x / D, y^{*}=y / D\right)$. Vorticity is normalized by the free-stream speed $U$ and the cylinder diameter $\left(\omega^{*}=\omega \frac{D}{U}\right)$.

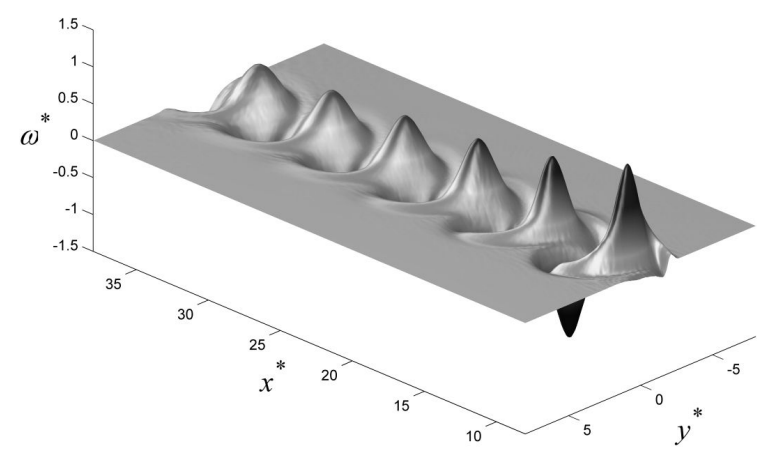

(a)

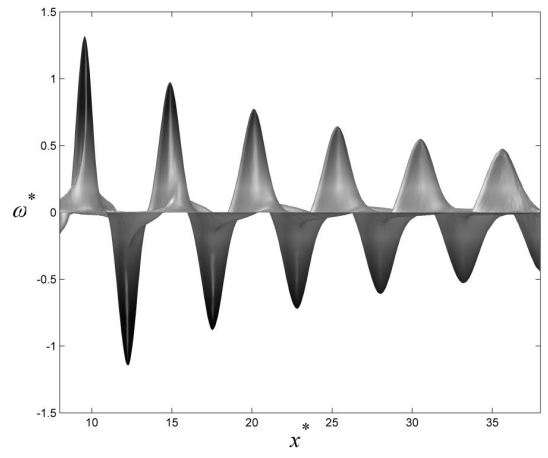

(b)

Figure 2.6. Panel $(a)$ : Three-dimensional perspective view of the vorticity distribution of a twelvevortex series in a Kármán-street wake for $\operatorname{Re}=100$. Panel $(b)$ : Lateral view of the same vorticity distribution, [Figure created by author from data in 8].

The two tails phenomenon mentioned earlier is displayed here. The severed outer appendage is only seen in the first vortex, which is in the final stages of the rearrangement process. This appendage is also seen weakly, in the second vortex but it is already partially incorporated. The inner appendage manages to last further downstream. 


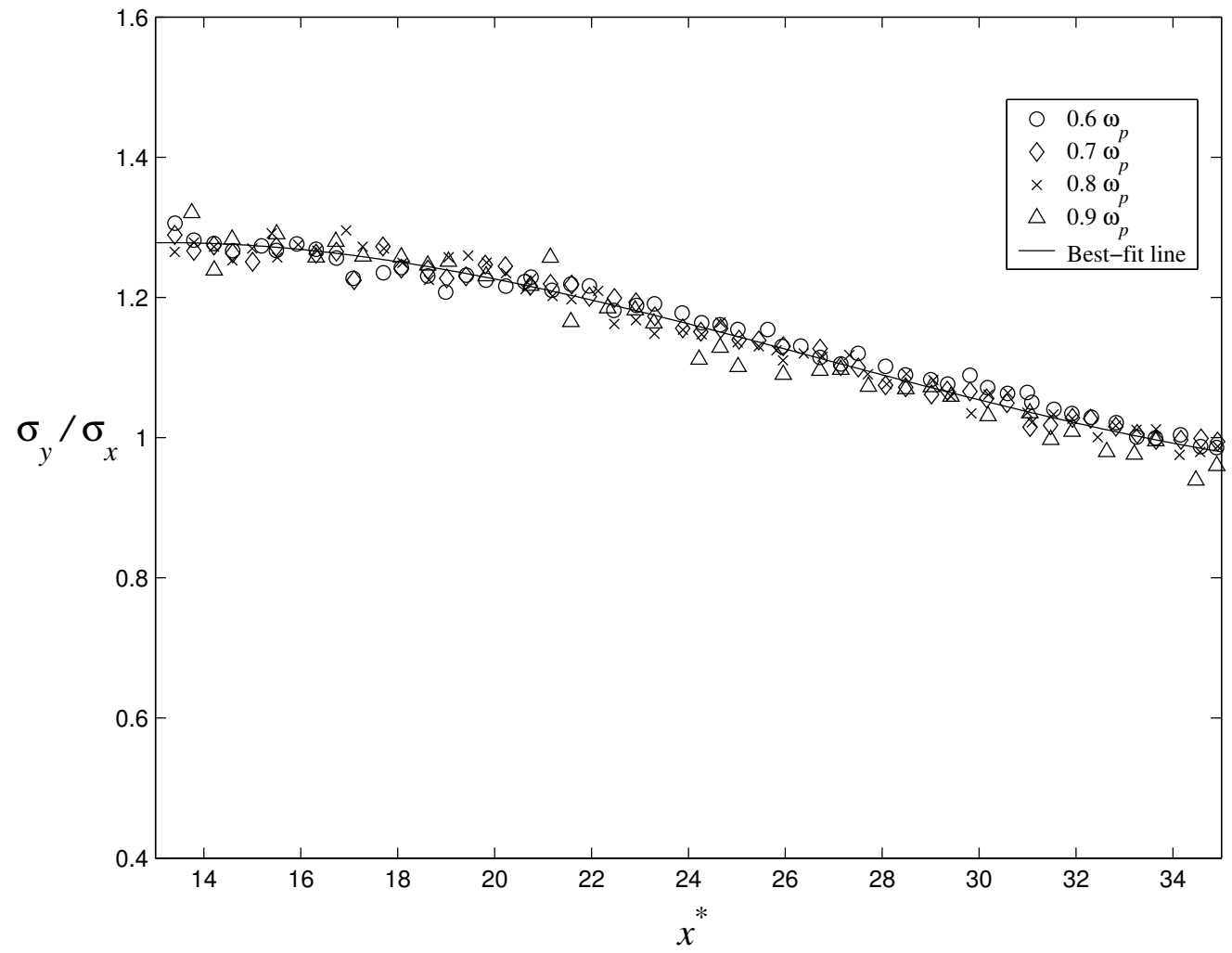

Figure 2.7. Elliptical ratio for several plane sections of the vortex core corresponding to horizontal slicing planes located at $0.6,0.7,0.8$ and 0.9 of $\omega_{p}$ and best-fit line, [Figure created by author from data in 8].

Despite the tails, the majority of the vorticity is enclosed in the core of each vortex. This vorticity inside each core is responsible for the structure of the wake via its role as the principal component of the induced velocity. This set of cores follow a Gaussian distribution. Each core displays an elliptical boundary with the major axis aligned vertically, normal to the inlet. The major to minor axis ratio $\left(\sigma_{y} / \sigma_{x}\right)$ is constant and is maintained as the vortex develops downstream. Ponta [8] has calculated $\sigma_{y} / \sigma_{x}$ for several plainer sections at $0.6,0.7,0.8$ and 0.9 of the maximum vorticity $\left(\omega_{p}\right) .0 .6 \omega_{p}$ was selected as a starting point of the section where the tails could be clearly seen. In other sections the tails would have appeared as a peak of the upper end of the elliptical contour of the cross section increasing and distorting the major-axis length. The evolution of $\sigma_{y} / \sigma_{x}$ as the vortex moves downstream can be seen in Figure 2.7. The curves of the various measurement planes converge around a single uniform function, which when expressed as a best-fit line is $\sigma_{y} / \sigma_{x}=3.36510^{-5}\left(x^{*}\right)^{3}-2.710^{-3}\left(x^{*}\right)^{2}+5.3810^{-2} x^{*}+0.961$. Comparing the vorticity profiles to a mathematically constructed set of Gaussian curves, which are a function of the $\sigma_{y} / \sigma_{x}$ ratio, a similar result is found. 
Going further, the underlying function that controls the rate of decay of the vortices was discussed by Ponta [8]. A 3-D lateral view of a twelve-vortex series vorticity distribution is seen in Figure 2.6 (b), this is the same data-set seen in Figure 2.6 (a). Based on observation of this plain $\omega_{p}^{*}=\omega_{p} \frac{D}{U}$ follows a systematic decreasing law which must be related to some vorticity distribution within the vortex cores. At about 15 diameters downstream of the cylinder the vortex cores start to overlap. Even here, the law is still valid and consistent with independent observations [48]. These observations were made using interferometric flowvisualization which found the dissipation is almost negligible up to $x / D=15$. Beyond this a rapid dissipation is caused by the overlap of adjacent viscous eddy cores and the cancellation of vorticity rotating in opposite directions.

Figure 2.8 shows the measurement of the decay of $\omega_{p}^{*}$ along the streamwise axis. The curve is nearly a perfect hyperbola $\left(\omega_{p}^{*}=19.579\left(x^{*}+5.259\right)^{-1}\right)$, also shown in Figure 2.8 [8]. This matches the decay law given by the Lamb's solution from expressions 2.3) to (2.4, where peak vorticity is given by

$$
\omega_{p}=\frac{\Gamma}{4 \pi \nu t},
$$

when normalized by the cylinder diameter $D$ and the free-stream speed $U$ yields

$$
\omega_{p}^{*}=\omega_{p} \frac{D}{U}=\frac{\Gamma}{4 \pi \nu t} \frac{D}{U}
$$

Since the speed of propagation of the wake is nearly constant, $t=\frac{x-x_{0}}{U_{w}}$ can be defined. $U_{w}$ is the speed of propagation of the wake and $x_{0}$ is a hypothetical point upstream of the cylinder where the hyperbola can be imagined to have an origin. Now, (2.8) can be rewritten in terms of the distance downstream $(x)$ instead of $t$

$$
\omega_{p}^{*}=\frac{\Gamma}{4 \pi \nu} \frac{D}{U} \frac{U_{w}}{x-x_{0}},
$$

when normalized by $D$ and $U$ yields

$$
\omega_{p}^{*}=\frac{R e}{4 \pi} \Gamma^{*} U_{w}^{*}\left(x^{*}-x_{0}^{*}\right)^{-1},
$$

where the normalized counter parts of the relevant variables are $\Gamma^{*}=\frac{\Gamma}{D U}, U_{w}^{*}=U_{w} / U$ and $x_{0}^{*}=x_{0} / D$. Lamb's solution proposes the vortices have a fixed amount of circulation that dissipates as the vortex ages. Between $x^{*} \approx 13$ and $x^{*} \approx 15$, the sum of the circulation can be calculated by direct integration of the vorticity contained in the eddy core. In this 


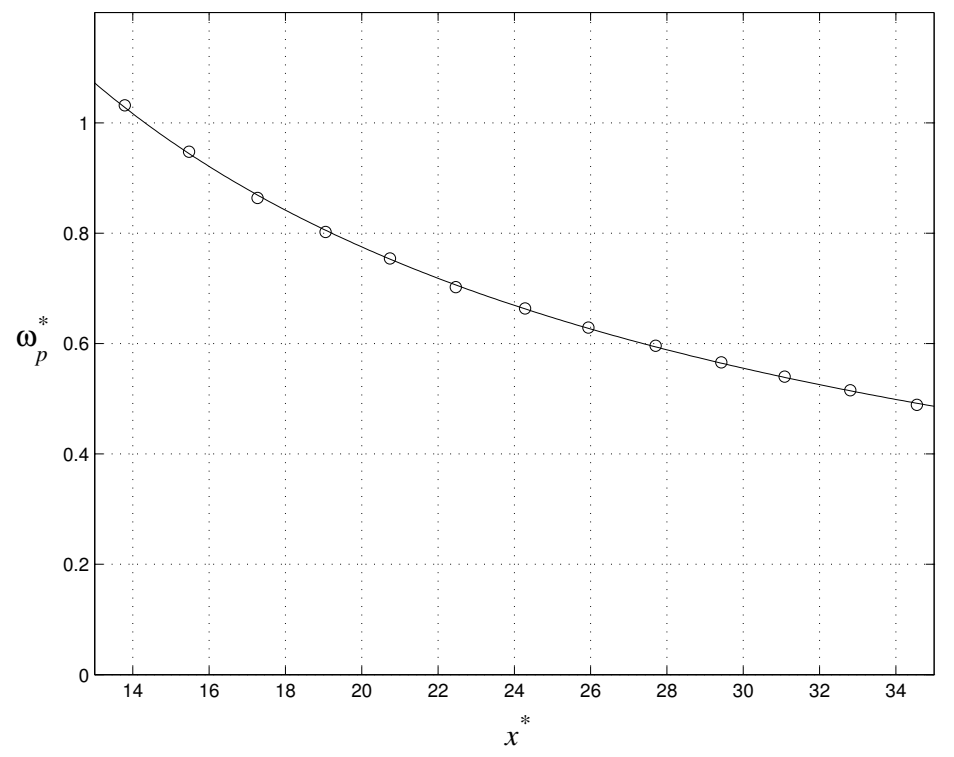

Figure 2.8. Decay of $\omega_{p}^{*}$ along the streamwise axis and best-fit hyperbola $\omega_{p}^{*}=19.579\left(x^{*}+\right.$ $5.259)^{-1}$, [Figure created by author from data in 8 ]. 
downstream section the eddy core is in the early stages of the street before the cores start to overlap but after the end of the rearrangement process. The limiting point of the integration was selected as the closed streamline which passes through the confluence point. This allows a well defined boundary of the vorticity core in the calculation. This calculation was repeated for multiple cycles and the result was $\Gamma^{*}=2.8 \pm 0.02$. A simple calculation can be used to find the propagation speed, which was $U_{w}^{*}=0.88$. Returning to the best fit hyperbola from Figure 2.8 is

$$
\omega_{p}^{*}=19.579\left(x^{*}+5.259\right)^{-1} .
$$

Equated to (2.10), the hyperbola above becomes

$$
\Gamma^{*}=19.579 \frac{4 \pi}{\operatorname{Re} U_{w}^{*}},
$$

which when solved results in $\Gamma^{*}=2.796$. This is essentially equal to the value of $\Gamma^{*}$ calculated by the integration of the cores. The amount of circulation contained in the cores at the boundary of the vortex street determines the law of decay for the vortices along the wake. This law closely matches Lamb's implicit solution.

Continuing in this direction, to remain consistent with the Lamb solution the vorticity profiles of the cores should be Gaussian, despite the fact the plainer sections are elliptical as opposed to circular. When (2.3) is rewritten in terms of an equivalent radius $R=2 \sqrt{\nu t}$, which was selected at the width of the two-dimensional circular Gaussian distribution, it is found

$$
\omega=\frac{\Gamma}{\pi R^{2}} e^{-r^{2} / R^{2}}
$$

Then (2.13) can be rewritten in terms of an elliptical Gaussian as opposed to a circular Gaussian

$$
\omega=\frac{\Gamma}{\pi R_{x} R_{y}} e^{-\left(\frac{x^{2}}{R_{x}^{2}}+\frac{y^{2}}{R_{y}^{2}}\right)},
$$

assuming $R_{x} R_{y}=R^{2}=4 \nu t$. That is, as long as the area under the Gaussian surface is preserved. 


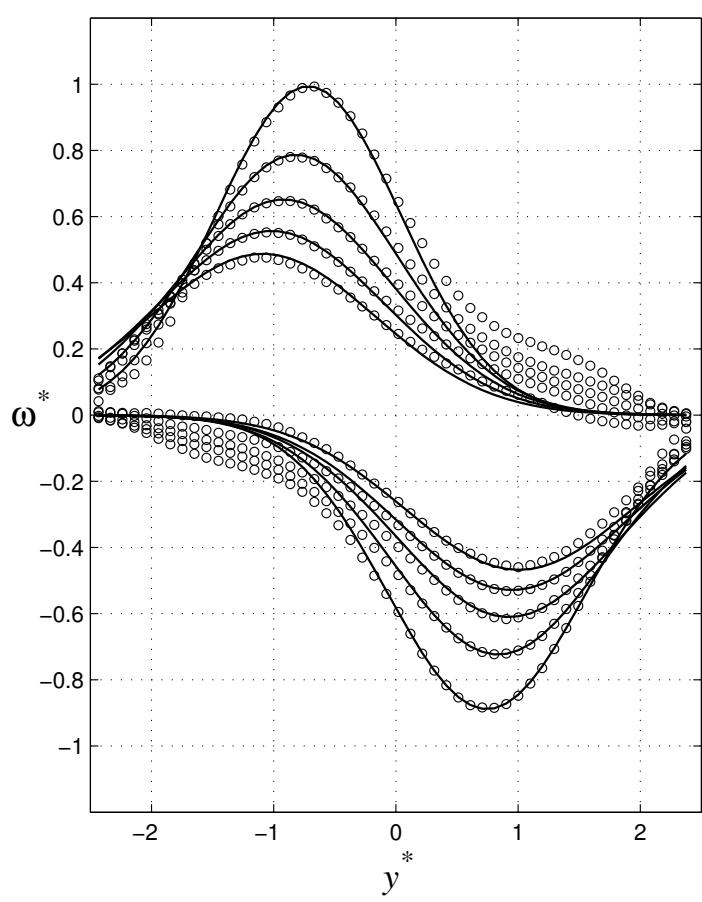

(a)

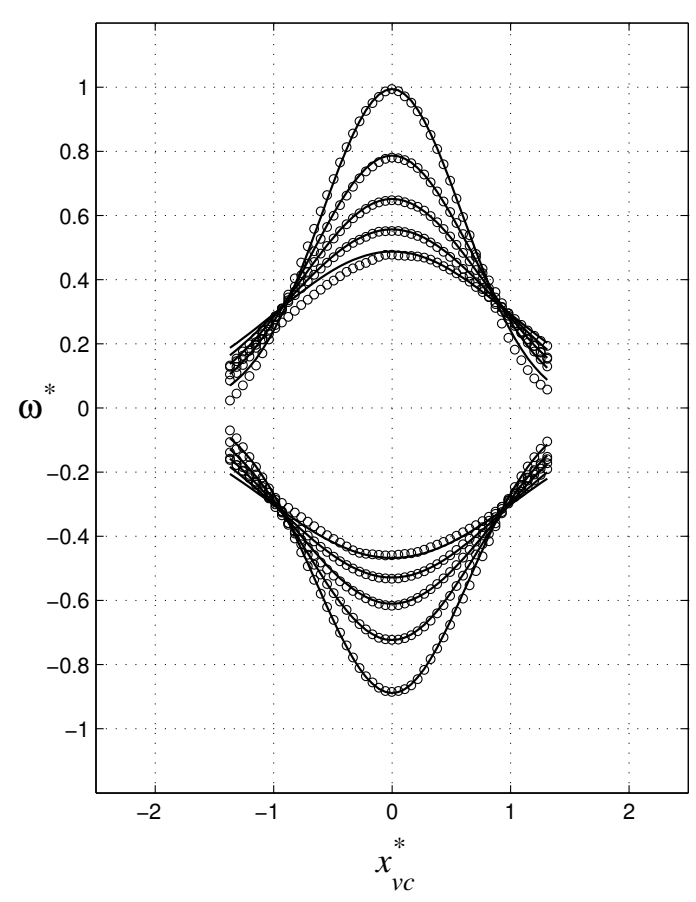

(b)

Figure 2.9. Panel (a): Comparison of the vorticity profiles in a cross-flow slicing plane for ten consecutive vortices. $y^{*}$ indicates, as hitherto, the non-dimensional cross-flow distance taken from the origin. Panel $(b)$ : Comparison of the vorticity profiles in a streamwise slicing plane for ten consecutive vortices. $x_{v c}^{*}$ indicates the non-dimensional streamwise distance taken from the vortex centre, [Figure created by author from data in 8].

In the derivation of (2.14), calculated values of $\Gamma^{*}, U_{w}^{*}$ and the elliptical ratio $\sigma_{y} / \sigma_{x}$ given in Figure 2.7 were used. This expression is used to describe the vorticity profiles. The ratio of $R_{y} / R_{x}$ is expected to match the ratio of $\sigma_{y} / \sigma_{x}$. A comparison between the non-dimensional vorticity profiles in plainer sections aligned to the cross-flow and the streamwise axis for a row of ten consecutive vortices can be seen in Figure 2.9. Both the cores and the tails are shown. The plainer sections pass through the peak vorticity at each vortex. The values determined by direct numerical simulations are the circular points while the Gaussian curves derived from (2.14) are represented by solid lines.

An important note on Figure 2.9, the Gausian curves are not fittings, but mathematically constructed from the calculated parameters discussed above [8]. In Figure 2.8, the value $x_{0}^{*}=-5.259$, representing the horizontal translation of the decay curve, is the only parameter taken from a curve fitting operation.

The Lamb-vortex model and the $(\omega-\psi)$ scatter-plots 
Due to the coincidence of the $\boldsymbol{v}_{\omega}$ streamlines and the vorticity isolines, the advective term in the vorticity transport equation is canceled out. This functional relationship $F$ between $\omega$ and the $\boldsymbol{v}_{\omega}$, represented by its associated stream function $\psi_{\omega}$, can be shown to be

$$
\omega=F\left(\psi_{v}\right)
$$

Based on what has been presented above, the vorticity is expected to evolve according to the Lamb solution. The vorticity profiles inside the cores would also be expected to be consistent with the Gaussian distribution of the Lamb-vortex model. The next logical step is to assume $\omega$ is related to $\psi_{\omega}$ by the corresponding functional relation. By integrating (2.4), the stream function in the Lamb-vortex model can be found to be

$$
\psi=-\frac{\Gamma}{2 \pi}\left(\frac{1}{2} \operatorname{Ei}\left(\frac{r^{2}}{4 \nu t}\right)+\log (r)\right)+\text { const. }
$$

where the exponential integral function $\mathrm{Ei}$ is

$$
\operatorname{Ei}(-z)=-\int_{z}^{\infty} \frac{e^{-z}}{z} \mathrm{~d} z, \quad(z>0) .
$$

Here, (2.16) assumes an isolated circular vortex. This allows the isolines for $\omega$ and $\psi$ to be coincident. It is clear this assumption does not capture the true behavior in the Kármán steet. In reality the vortices in question have overlapping elliptical cores and tails that form due to escaping traces of vorticity. Despite this the core contains the bulk of the vorticity which induces the dominant component of $\boldsymbol{v}_{\omega}$. The traces of vorticity that forms the tails seem to dissipate with minimal impact to the wake structure. Even considering elliptical vortex cores, the Lamb vortex functional relation is still valid. A change of coordinates by the deformation in the wake axis may reproduce the elliptical shape and will not alter the functional relation $F$.

To produce the $\omega-\psi$ scatter plot shown below, the values for $\omega$ and $\psi$ are taken from every point of a grid that covers the region of the wake in question. This gives a useful representation of $F$. This technique was used by multiple researchers, including Flór and van Heijst [49] to analyze the time and structure evolution of monopolar vortices, dipoles and tripoles. Flór and van Heijst [49] described the flow where the reference point translates with the dipole, this translation follows the path where the isolines of $\omega$ and $\psi$ are coincident and a discernible functional relation may exist. This path was determined by plotting $\omega$ opposed to a transformed stream function $\psi^{\prime}=\psi-U_{x} y+U_{y} x$, when the coordinate $\left(U_{x}, U_{y}\right)$ is the translational velocity of the dipole. In Ponta's work, $\psi_{\omega}$ is equivalent to the 
transformed $\psi^{\prime}$ in the referenced work above. The incompressible velocity field $\boldsymbol{v}$ can be decomposed and be re-written in terms of the velocity potentials:

$$
\boldsymbol{v}=\boldsymbol{v}_{\omega}+\boldsymbol{v}_{h}=\boldsymbol{\nabla} \times \boldsymbol{\psi}_{\omega}+\boldsymbol{\nabla} \times \boldsymbol{\psi}_{h},
$$

where the stream vector potentials associated with $\boldsymbol{v}_{\omega}$ and $\boldsymbol{v}_{h}$ are $\boldsymbol{\psi}_{\omega}$ and $\boldsymbol{\psi}_{h}$ respectively. When applied to two dimensional cases, this reduces to another component given by the stream vector potentials. The Poisson equation for related to $\psi_{\omega}$ would then be

$$
\nabla^{2} \psi_{\omega}=-\omega
$$

This is satisfied within any harmonic component. In Ponta's work, the stream function associated with $\boldsymbol{v}_{\omega}$ was evaluated independently. Then $\boldsymbol{v}_{\omega}$ can be uniquely determined by evaluating (2.19) using the same spatial discretization described earlier for the velocity field and adding the constraint (2.20), where

$$
\nabla \times \boldsymbol{\psi}_{\omega}=\boldsymbol{v}_{\omega}
$$

In Figure 2.10 is a 3-D perspective view of $\psi_{\omega}$ for the twelve-vortex wake at $\operatorname{Re}=100$, shown earlier in Figure 2.6 [8]. The Stream function is normalized by the free stream speed and the cylinder diameter $\psi_{\omega}^{*}=\psi_{\omega} \frac{D^{2}}{U}$ ).

In Figure 2.11 is a scatter plot of $\omega^{*}-\psi_{\omega}^{*}$ for a ten-vortex wake where $\operatorname{Re}=100$. Here only the cores of the vortices are shown. The figure clearly shows the $\omega-\psi$ pattern for the five negative and five positive vortices. Unlike [49] the normalization of the values of $\omega$ and $\psi$ were taken by a standard normalization based on the free stream speed and the cylinder diameter as opposed to the respective maximums. The first and third of the five vortex pairs from Figure 2.11, including cores and tails in this case, are depicted in Figures $2.12(a)$ and $2.12(b)$ respectively. The solid lines in this figure represent the theoretical $\omega-\psi$ curve for a Lamb vortex in this position downstream of the origin. It is important to note, these curves are not best fit curves but calculated results derived from (2.3) and (2.16). The value of the circulation of the vortex was obtained by integrating the early stages of the vortex street. The only fitted value that is used is the horizontal translation of the $\omega-\psi$ curve which is the integration constant in 2.16). It is then seen that as the vortices progress downstream, they diverge from the center and their cores overlap. This then forces the $\omega^{*}-\psi_{\omega}^{*}$ pattern to widen. With this considered, the dominant structure is still the $\omega-\psi$ functional relation described by the Lamb-vortex model. 


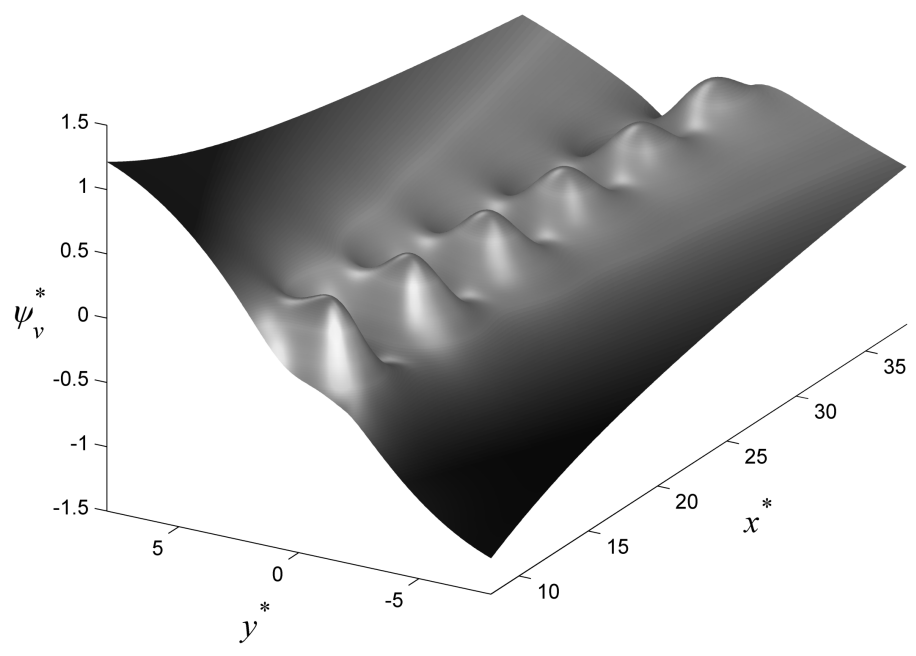

Figure 2.10. Three-dimensional perspective view of the $\psi_{\omega}$ distribution corresponding to the Kármán-street wake depicted in figure 2.6, [Figure created by author from data in 8]

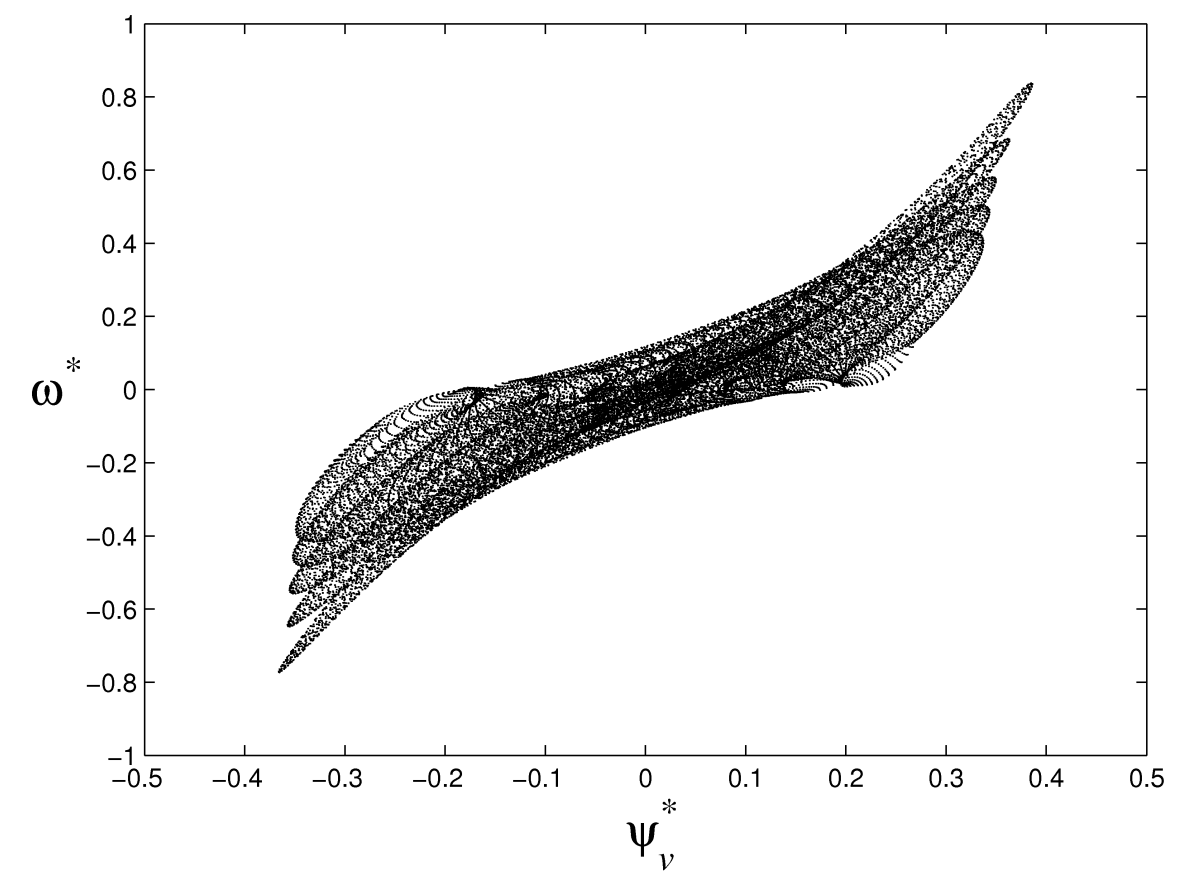

Figure 2.11. $\omega^{*}-\psi_{\omega}^{*}$ scatter plot for a complete ten-vortex wake at $R e=100$, [Figure created by author from data in $[8]$. 


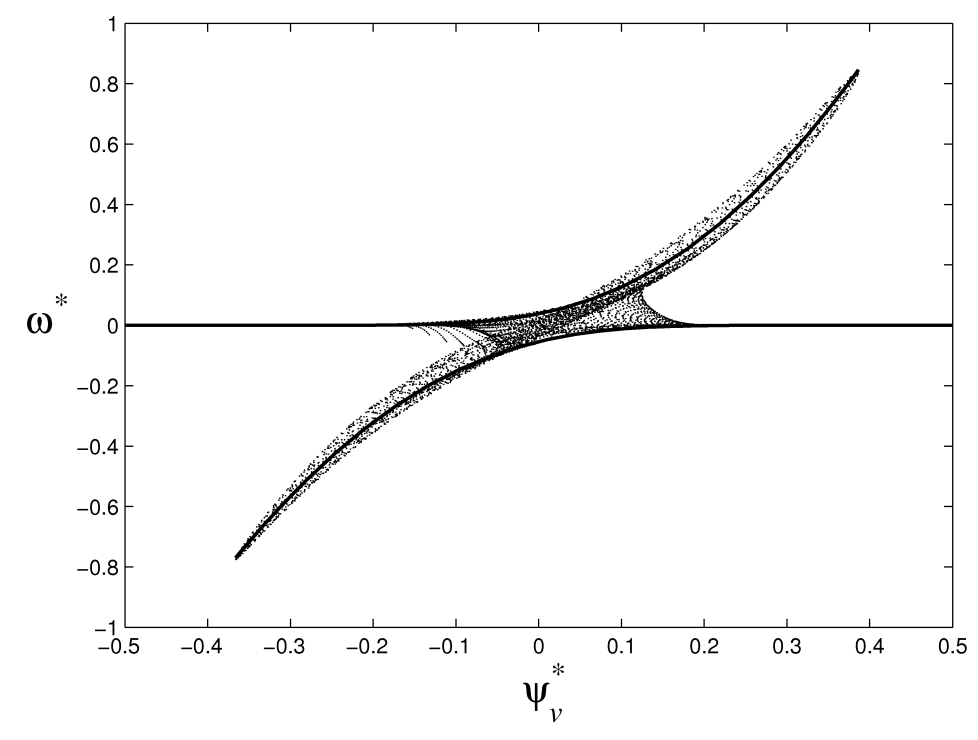

(a)

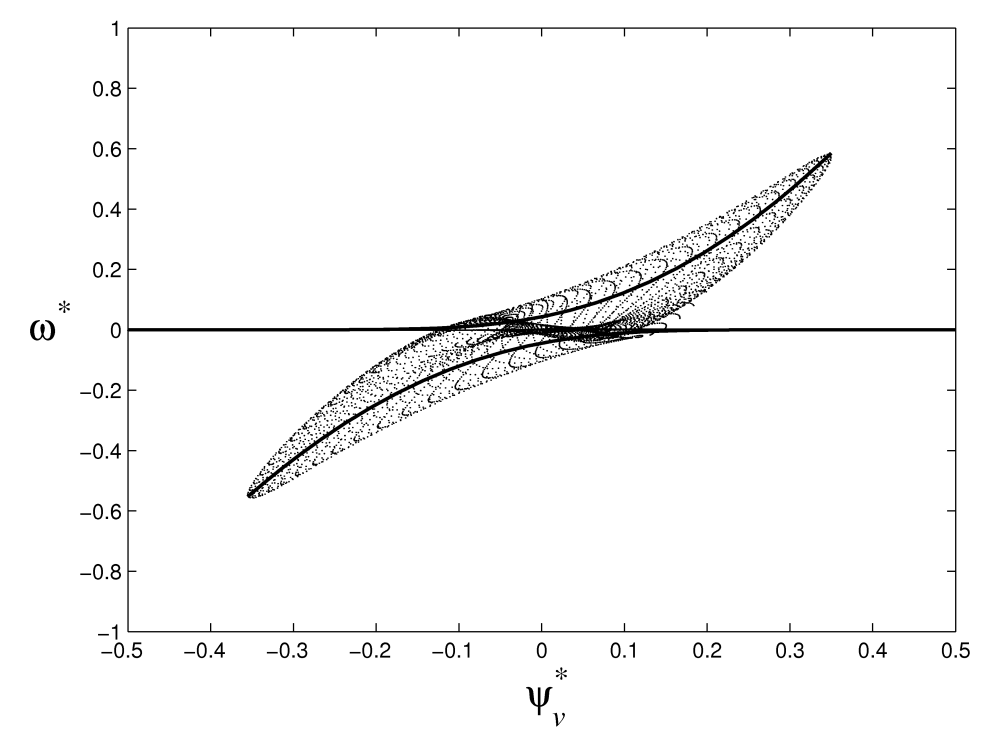

(b)

Figure 2.12. $\omega^{*}-\psi_{\omega}^{*}$ scatter plots for the first and the third of the five vortex pairs in the wake depicted in figure 2.11] [Figure created by author from data in 8]. The solid lines correspond to the theoretical $\omega-\psi$ curve for a Lamb vortex located at the same distance downstream. 


\subsection{Wake behind oscillating cylinders}

The vortex wake patterns of two opposite vortices per shedding cycle are very limited. They are basically different versions of a Kármán vortex street with varying ratio of spacings between inter/intra rows. As known, an oscillating body produce a more complex vortex wake different from the classic Kármán vortex street, which would translate into a complex fluctuating aerodynamic force. A term "exotic wakes" has started being used for those patterns. This system can be considered as a forced, non-linear oscillator.

One may consider the shedding frequency associated with the free stream velocity as the system's natural frequency and the frequency of oscillating body as an external forcing frequency. A large number of combined frequencies can potentially be excited owing to the non-linear coupling between the two oscillations mentioned. Several of them may be related to new vortex wake patterns. Even in the cases of stationary vortex wake pattern, it is more likely to have three rather than two vortices in the wakes for each shedding cycle. Stationary wake patterns means that the configuration of the vortices produced does not change between two subsequent cycles of oscillation and shedding. Given the assumption of point vortices, these patterns have been thoroughly classified by [50].

A substantial amount of literature has been published on experimental research of vortex wakes behind oscillating cylinders subject to oscillations either normal to or along the uniform stream (see [51], among others). Besides these, there are more recent theoreti$\mathrm{cal} /$ numerical studies (see [52] for further references).

Numerous researchers have demonstrated and analyzed the occurrence of these complex wakes behind circular cylinders that oscillate on a background flow (see [9, 10,51,53,54, among others). Williamson \& Roshko (referred to as WR hitherto)[9] provide an experimental bifurcation diagram for wakes produced by an oscillating cylinder.

WR constructed a map of vortex synchronization regions and the coordinates in the map are, $\lambda / D$, the Non-dimensional wavelength, and $A / D$, Non-dimensional amplitude of cylinder oscillations, where $\lambda$ is the wavelength and $A$ amplitude of oscillations. An alphanumeric code system was created by WR to describe the patterns of vortices shed in various combinations comprising of single and paired vortices in each cycle. These symbols have become a standard classification for these structures of the forced oscillation of the cylinder. WR partitioned the map into different synchronization regions based on the prevalence of patterns in the vortex wakes. They denoted these patterns by the alphanumeric code they had developed. For example a $\mathrm{P}+\mathrm{S}$ would mean a shedding pattern comprising of an oppositely signed vortex pair and a single vortex per shedding cycle. Similarly $2 \mathrm{P}$ would mean two oppositely signed pairs and $2 \mathrm{~S}$ would signify 2 single vortices being 
shed in each cycle. 'S' stands for a single vortex while 'P' for a pair of oppositely signed vortices.

Several features in the Williamson \& Roshko [9] vortex synchronization map were rationalized by Ponta \& Aref [11] using physical arguments based on how the oscillating trajectory followed by the body motion affects the timing of vortex shedding and re-arrangement. Simulations as well as experiments suggest that the final wake configuration is produced by a combination of splitting and merging. In the near-wake region, these processes change the strength as well as the number and position of vortex structures that form the vortex street (or whatever the final pattern is).

A particular detailed study at $R e=392$ by WR reported that regions $\mathrm{P}+\mathrm{S}$ and $2 \mathrm{P}$ dominated the $(\lambda / D, A / D)$ map vortex synchronization regions $\mathrm{P}+\mathrm{S}$ and $2 \mathrm{P}$. WR noted that the boundary between those vortex synchronization regions depend on $R e$. Therefore at $R e>300$ a $2 \mathrm{P}$ region takes over some of $\mathrm{P}+\mathrm{S}$ at $R e>300$. Ponta performed numerical experiments at $R e<300$ exclusively [10] and found the $2 \mathrm{P}$ region starts shrinking or even vanishes. It is difficult to determine the boundaries between the various regions experimentally or even numerically since the theory driving them is still not as clear. Nevertheless, Ponta proposed an argument to mark out boundaries on the WR map similar to the ones already observed. This might help explain the shifting of regions with change in Re.

\subsubsection{Boundaries marking vortex synchronization regions}

WR saw that the $(\lambda / D, A / D)$ map can accurately represent the synchronization regions throughout the range of $300<R e<1000$. For the same rang of Reynolds numbers, the Strouhal number for a non-oscillating cylinder remains nearly constant. For $R e>300$ the $S t-R e$ curve asymptotically approaches an approximate constant value of $S t=0.2$. Thus, the natural frequency of the system in the oscillator analogy is largely fixed while the forcing frequency alone varies. For a fixed Strouhal number equal to 0.20 within $300<$ $R e<1000$, it is possible to correlate $\lambda / D$ with the ratio of the time period of forced oscillations $\left(T_{e}=\lambda / U\right)$ and that of the shedding of vortices for a non-oscillating cylinder $\left(T_{s}=D /(U S t)\right)$, via $T_{e} / T_{s}=S t \lambda / D=0.20 \lambda / D$. This correspondence allows $\lambda / D$ to be considered as a scaled down Time period $T_{e}$ in terms of time period of the system's natural oscillation (which is the same as the period of shedding of a Kármán vortex street in case of a non-oscillating cylinder).

However, according to Ponta's numerical simulations for $R e<300$, specifically for $R e=140$, the most studied case, the frequency at which the Kármán vortex street is shed varies considerably with Reynolds number. For an oscillating cylinder moving upstream with a constant horizontal the relative velocity of the cylinder varies thereby causing the 
cylinder $R e$ to fluctuate. For $R e<300$, a marked dependence of Strouhal number suggests considerable variation in the time period of natural oscillation. Also, the amplitude at which Re fluctuates is dependent on the non-dimensional parameters $\lambda / D$ and $A / D$, thereby changing all over the WR map [11].

Considering the center of the cylinder moves as follows:

$$
\begin{aligned}
& x_{c y}(t)=U t \\
& y_{c y}(t)=A \sin \left(2 \pi \frac{t}{T_{e}}\right)=A \sin \left(2 \pi \frac{t U}{\lambda}\right) .
\end{aligned}
$$

Then, the relevant velocities are

$$
\begin{aligned}
& V_{x c y}(t)=U, \\
& V_{y c y}(t)=2 \pi U \frac{A}{\lambda} \cos \left(2 \pi \frac{t U}{\lambda}\right) .
\end{aligned}
$$

The maximum cylinder velocity along its trajectory is

$$
\hat{U}=U \sqrt{1+\left(2 \pi \frac{A}{\lambda}\right)^{2}}
$$

And the maximum associated $R e$ is,

$$
\hat{R} e=\frac{D}{\nu} U \sqrt{1+\left(2 \pi \frac{A}{\lambda}\right)^{2}}=R e \sqrt{1+\left(2 \pi \frac{A}{\lambda}\right)^{2}} .
$$

The difference between instantaneous period of vortex shedding, $T_{s}$, and its corresponding value at maximum $R e$, with respect to, $T_{e}=\frac{\lambda}{U}$, is 


$$
\Delta=\frac{T_{s}-\hat{T}_{s}}{T_{e}}=\frac{\frac{D}{U S t}-\frac{D}{U \hat{S t} t}}{\frac{\lambda}{U}}=\frac{1 / S t-1 / \hat{S t}}{\lambda / D},
$$

Here $\hat{T}_{s}$ is the period of shedding of vortices associated with $\hat{R e}$ and $\hat{S} t$ is the Strouhal number for that $\hat{R} e$. Taking the experimental best-fit line of $S t-R e$ relation summarized by Roshko [55],

$$
S t= \begin{cases}0.212(1-21.1 / R e), & R e<180 \\ 0.212(1-12.7 / R e), & R e>300\end{cases}
$$

substituting it into (2.27) and then using (2.26), it gives $\Delta$ in terms of the Reynold's number and the parameters of the WR map:

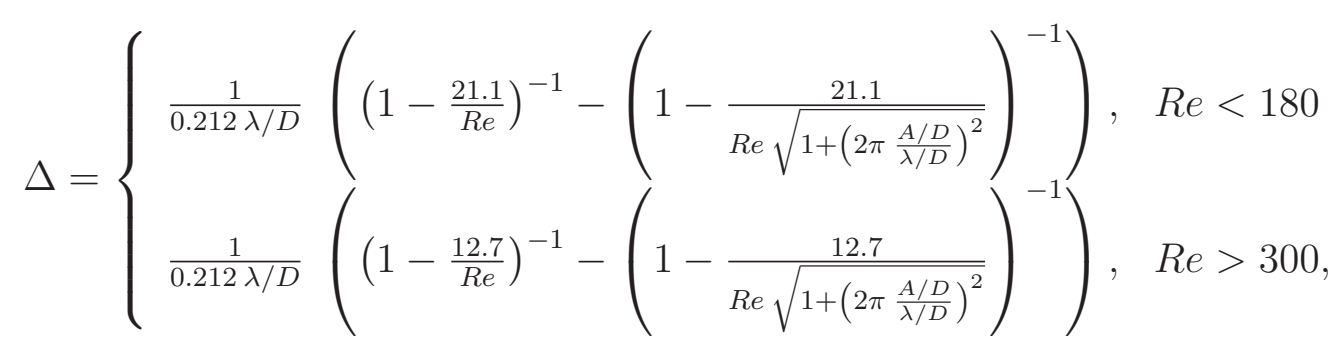

Figure $2.13(a)$ is the map as presented by WR superimposed with several values of $\Delta$ for $R e=392$. These $\Delta$ are presented with the $0.001,0.015$ and 0.06 isolines and $R e=392$ is the representative case for the $R e$ range where the map is valid. As shown in the figure, these isolines coincide roughly with the boundaries of region $\mathrm{P}+\mathrm{S}$ and region $2 \mathrm{P}$ [11]. Ponta suggested that, due to this coincidence, the boundaries of the vortex synchronization regions might be related to fixed levels of fluctuation in the cylinder's natural shedding frequency. These frequency fluctuations are result of the varying relative velocity of cylinder with respect to the fluid, owing to oscillatory motion.

Figure $2.13(b)$ presents the WR map but superimposed with several isolines of $\Delta$ in the case of $R e=140$. The vortex synchronization region between the isolines of $\Delta=0.015$ and $\Delta=0.06(\mathrm{P}+\mathrm{S}$ region in the original WR map), after rotating down to the right in the clockwise direction, occupies the previous $2 \mathrm{P}$ region. Furthermore, the region between the isolines of $\Delta=0.001$ and $\Delta=0.015$, associated with previous $2 \mathrm{P}$ states, has shifted down so much that it almost no longer occupies the region in which $2 \mathrm{P}$ patterns were seen earlier. Hence, assuming that boundaries of the vortex synchronization region are related 
to isolines of $\Delta$, it is possible to explain why at low $R e \mathrm{P}+\mathrm{S}$ state replaces the $2 \mathrm{P}$ state (which is no longer observed) [11]. Basically, the fluctuation of the shedding frequency is too high for a $2 \mathrm{P}$ pattern, which for some reason is a lot more reactive to detuning than $\mathrm{P}+\mathrm{S}$. Also, $S t$ reacts a lot more to changing $R e$ at lower Reynolds numbers than at higher ones. This argument seems to show some consistency with the thought that both $2 \mathrm{P}$ and $\mathrm{P}+\mathrm{S}$ have similar generation mechanisms, but a finer balance is required for a $2 \mathrm{P}$ wake than that for $\mathrm{P}+\mathrm{S}$. Experiments by WR show that owing to strain on each vortex of $\mathrm{P}$ by neighboring vortices a $2 \mathrm{P}$ wake is formed. Thus, it produces two vortex pairs in each cycle instead of one pair. As shown, in the $\mathrm{P}+\mathrm{S}$ wake, while positive as well as negative vortices are present in the near wake region, one splits by stretching but the other recovers and does not split producing only three vortices in each cycle. So for both $2 \mathrm{P}$ and $\mathrm{P}+\mathrm{S}$ splitting of vortices is fundamental to the formation of vortex wake structures, a finer synchronization between negative and positive vorticity regions might be required making it more sensitive to frequency and time scale variations.

As shown in figure 2.13, the boundaries between different synchronization regions can be approximately grouped into two families: the circumferential family characterized by a group of concentric-wise curves which appear to be roughly centred on the origin; and the radial family which corresponds to another group of curves that expands radially from the origin. Ponta and Aref [11] re-interpreted the boundaries between the synchronization regions belonging to the circumferential family in terms of a new parameter that also measures the ratio between the period of the forced and the natural oscillations. But instead of taking the wavelength $\lambda$ as the characteristic length, Ponta and Aref suggested adopting the length of the path for each cycle. Figure 2.14 shows the sinusoidal trajectory of the oscillating cylinder. It alternates sectors where the motion is quasi-rectilinear (marked $\mathrm{R}$ in the figure) with sectors with small radius of curvature where there are rapid changes for the angle of the inflow (marked C).

The vortex shedding process developed alongside the R-sectors of the path behaves roughly the same as a non-oscillating cylinder. On the other hand, as the inflow angle changes rapidly at peaks and troughs of the $\mathrm{C}$-sectors, points of separation change position interrupting vortex shedding, which resumes alongside the following R-sector [11]. Hence, if $\mathrm{R}$-sectors are one half the length of the natural wavelength (i.e. the distance covered by a non-oscillating cylinder in half a shedding cycle), shedding of a pair of equally-balanced cumuli of vorticity will occur per cycle of forced oscillation. Two pairs of cumuli will be shed if the length of the R-sector equals the double (instead of half), and so on. Ponta and Aref [11] proposed a hypothesis that the vortex synchronization regions of the $(\lambda / D, A / D)$ map corresponding to trajectories with length of R-sector equal to an integral multiple of half the natural wavelength define families of patterns of a common origin, namely, the same number of primarily shed vorticity cumuli. Variations seen in patterns of vortices belonging to the same family depend on the various processes that the primary vorticity cumuli went through after shedding. Experimental observations confirmed this argument. 


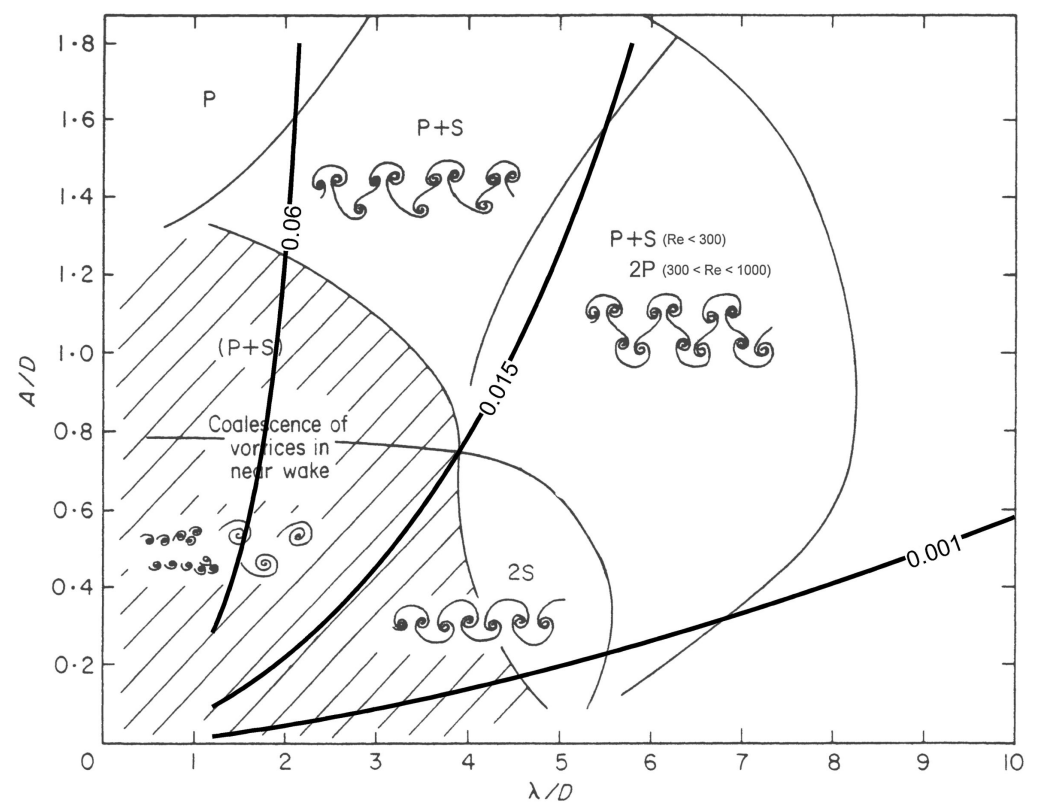

(a)

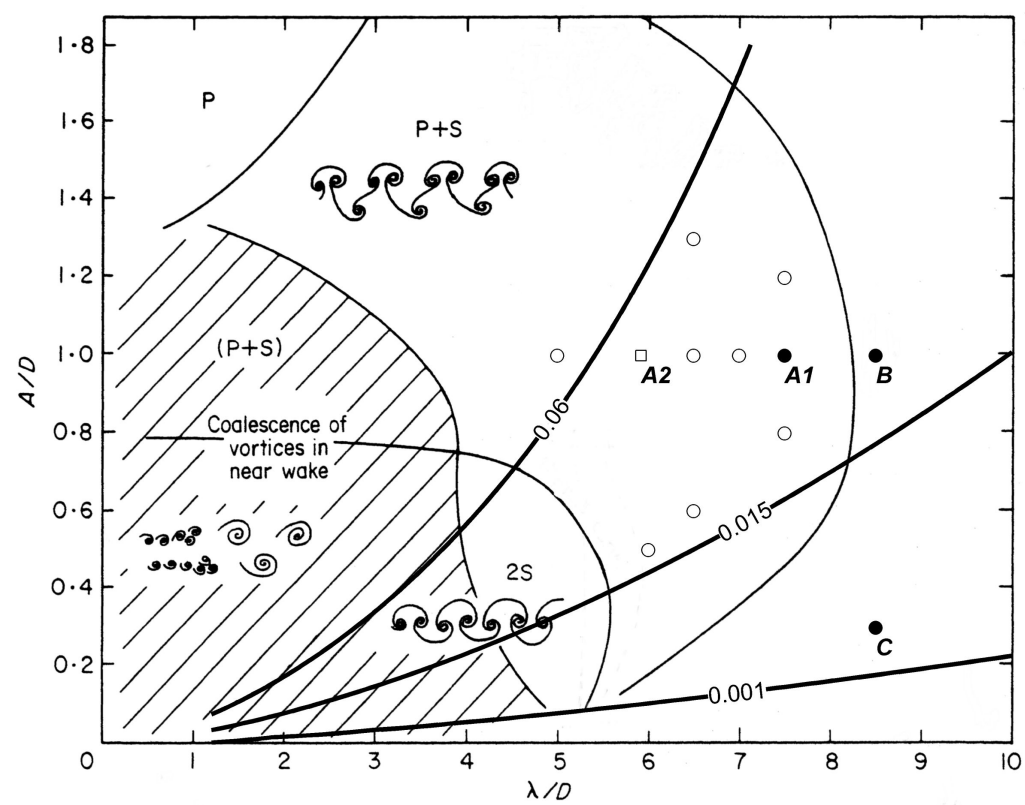

(b)

Figure 2.13. Original $(\lambda / D, A / D)$ map [9] with the numerical $0.001,0.015$ and 0.06 isolines of $\Delta$ (a) $R e=392(b) R e=140$. The Points of the parameters used in the numerical experiments are also shown in (b), [taken from 10]. See appendix for permission. 


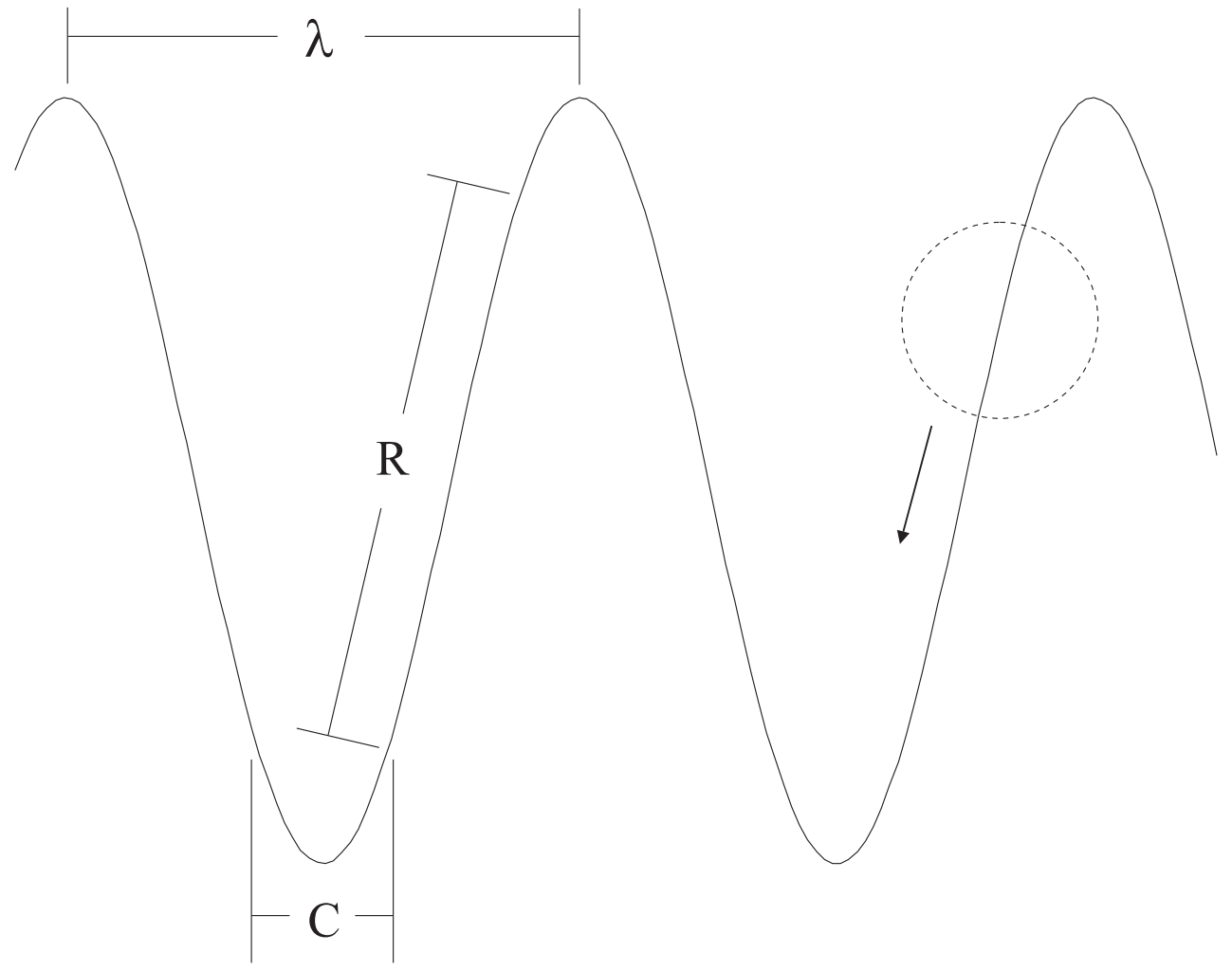

Figure 2.14. The sinusoidal trajectory of the oscillating cylinder, [Figure created by author from data in 11]. 
Govardhan and Williamson [53] indicated that a 2P wake arises from splitting both vorticity cumuli being shed, owing to the strain by the neighboring vortices. Therefore, it produces two vortex pairs in each cycle instead of one pair. In the $\mathrm{P}+\mathrm{S}$ wake, as explained earlier, inspite of both positive and negative cumuli in the end only three vortices are produced in each cycle. So, as mentioned before, splitting is fundamental to both $2 \mathrm{P}$ and $\mathrm{P}+\mathrm{S}$ wake formation, and even though the process of splitting is not the same for both, each involves the shedding of two cumuli of vorticity of either sign in every cycle. Thus the two are part of the same region as far as boundaries defined by the R-sector length hypothesis are concerned.

The R-sector length depends on the overall length of the path for one cycle. Hence, Ponta and Aref [11] defined a non-dimensional parameter $L_{D}=S t L / D$ (where $L$ is the path length for one cycle) which is essentially the ratio between the non-dimensional path length and the natural wavelength of the system given by $S t^{-1}$. The calculation of $L_{D}$ is simply the integral of the sinusoidal path length along one cycle,

$$
L_{D}=\frac{S t}{D} \int_{0}^{T_{e}} \sqrt{U^{2}+\left(2 \pi U \frac{A}{\lambda} \sin \left(2 \pi \frac{t U}{\lambda}\right)\right)^{2}} \mathrm{D} t,
$$

introducing a non-dimensional time $\hat{t}=t U / D$ as integration variable, it yields to

$$
L_{D}=S t \int_{0}^{\lambda / D} \sqrt{1+\left(2 \pi \frac{A / D}{\lambda / D} \sin \left(2 \pi \frac{\hat{t}}{\lambda / D}\right)\right)^{2}} \mathrm{D} \hat{t}
$$

which depends only on the parameters of the map $\lambda / D$ and $A / D$.

Following a similar line of reasoning, Ponta \&Aref proposed to re-interpret the boundaries between the synchronization regions belonging to the radial family in terms of another parameter that measures the level at which the natural frequency of shedding fluctuates along the cycle induced by the variation of the cylinder speed. As the cylinder oscillates while moving upstream with a constant velocity, it's relative velocity is changing, and hence the effective $R e$ along it's trajectory shows fluctuations with an amplitude which is a function of $\lambda / D$ and $A / D$. Hence, the fluctuation varies across the WR map, the consequences of which can be seen in the sinusoidal motion of the cylinder represented by equation 2.22 to equation 2.29 .

Williamson and Brown [56] put forward an accurate series in powers of $(1 / \sqrt{R e}), S t=$ $A+B / \sqrt{R e}+C / R e$ which could also be used to calculate the $S t$-Re relationship. They also compared different set of values for the coefficients according to several fits to numerical and experimental data. 

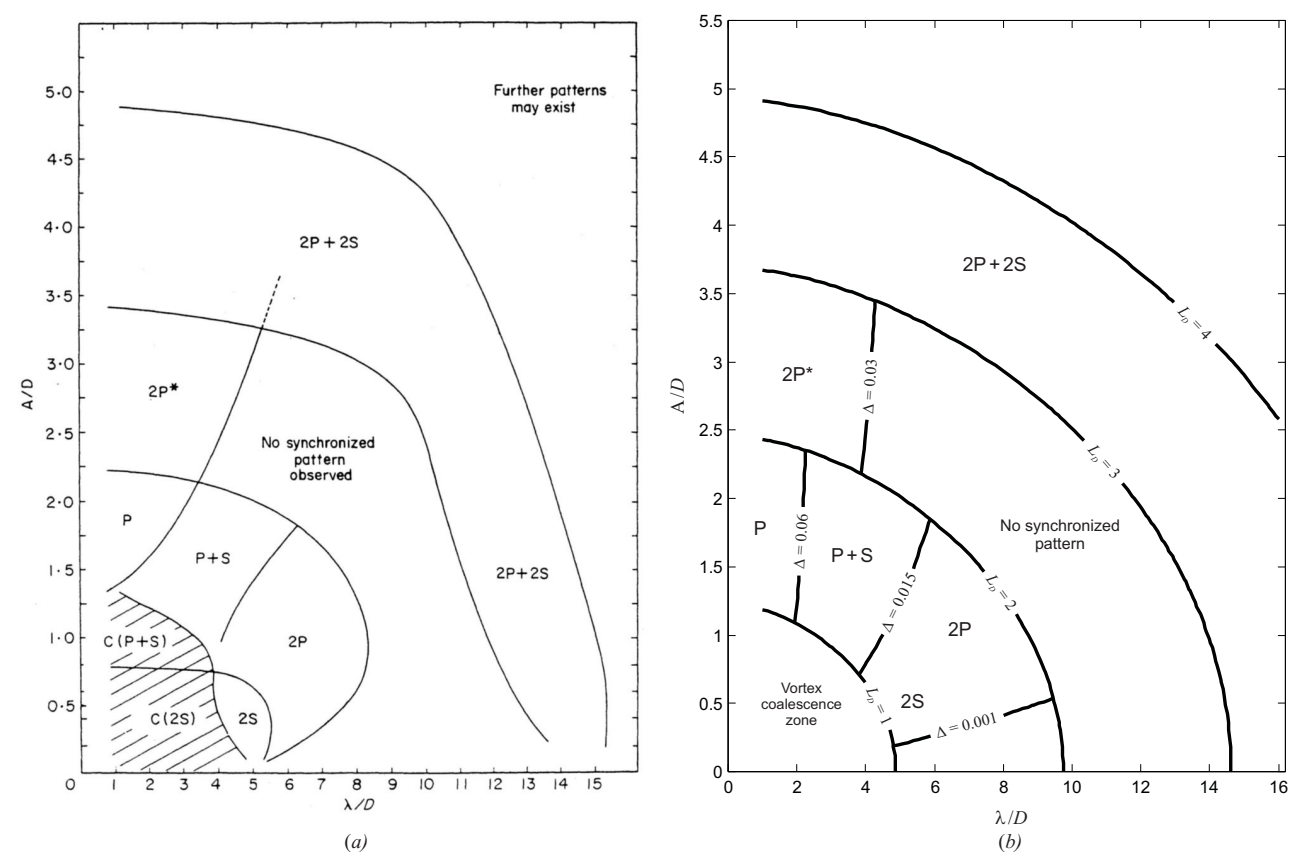

Figure 2.15. (a), Vortex synchronization regions for cylinders oscillating normally in the plane represented by amplitude and wavelength plane as seen in experiments by Williamson and Roshko [9], see appendix for permission, (b), synthetic version of the WR map with boundaries defined by the $L_{D}$ and $\Delta$ isolines, [Figure created by author from data in 11].

Figure 2.15 $(a)$ presents the $(\lambda / D, A / D)$ map of vortex synchronization regions for cylinders oscillating normal to the stream, obtained via experiments by Williamson and Roshko [9]. Figure 2.15 $(b)$ shows the synthetic version of the WR map where Ponta depicted the $L_{D}$ isolines for $1,2,3$ and 4 , and the isolines of $\Delta$ equal to $0.001,0.015,0.03$ and 0.06 at $R e=392[11]$.

In summary, Ponta and Aref brought forward a physical explanation on the occurrence of vortex patterns in the wake of cylinders undergoing forced oscillation. They have provided a theoretical version of the experimental WR map in the non-dimensional $(\lambda / D, A / D)$ plane, based on the assumption that the boundaries of these regions are grouped into two families of curves which are related with specific values of two non-dimensional parameters.

The suggestion that the regions enclosed by boundaries of the circumferential family correspond to families of patterns evolved from the same number of vorticity cumuli originally shed during the cycle is consistent with WR experimental observations [11]. The $2 \mathrm{P}$ and $\mathrm{P}+\mathrm{S}$ wake patterns evolve from the splitting of two vorticity cumuli whose circulation is balanced. The substantial agreement between the boundaries of the circumferential family 
on the experimental WR map and the distance traveled by the cylinder to produce integer numbers of vortices per cycle is significant. The vortex synchronization region with a label of 'No synchronized pattern observed' in the WR map refers to the disruption of the shedding process due to the variation in angle on the $\mathrm{C}$-sector inflow before the complete shedding of the cumuli. Thus, circulation in the cumuli in every shedding cycle of the forced oscillation is not the same as the amount of vorticity due to normal shedding from a non-oscillating cylinder. There, the cumuli are not balanced and undergo a series of complicated merges and splits leading to non-repetitive patterns that resemble a chaotic behavior on the oscillator analogy. The region in which WR saw coalescence into a largescale is highlighted with shades in Figure 2.15 $(a)$. First reported in Williamson \& Roshko [9], coalescence involves the merging of many small individual vortical structures shed at high-frequency, low-amplitude vibration states. The small vortices coalesce into substantially larger structures that arrange themselves into a huge, low-frequency vortex street of the hyper-Kármán kind.

As aforementioned, in $\mathrm{P}+\mathrm{S}$ and $2 \mathrm{P}$ wakes, splitting is the key to form structures of the wake. However, the influence of oppositely signed vorticity regions required for $2 \mathrm{P}$ pattern may need greater synchroneity making it respond more to time scale and frequency fluctuations. Ponta and Aref also reported some numerical experiments conducted by moving the cylinder along a constant-speed trajectory instead of the sinusoidal one. Adding a fluctuating component to the originally-constant horizontal motion, the cylinder keeps a constant speed along its path. Thus, the fluctuation of the instantaneous Reynolds number disappears. Even though the frequency and amplitude that produced the $\mathrm{P}+\mathrm{S}$ wake for the classical sinusoidal path remain the same, this constant-speed trajectory produced a symmetric wake where non-symmetrical splitting completely disappeared Ponta and Aref [11]. This result verifies the argument that the fluctuating velocities are key to the whole process of splitting affecting its symmetry.

\subsubsection{On P+S wakes forming at low Reynolds number}

The various parameter sets shown in Figure 2.13 $(b)$ were explored by Ponta and Aref. Among them, the clearest $\mathrm{P}+\mathrm{S}$ arrangement was generated at point $A 1$ at $(\lambda / D=7.5$, $A / D=1)$. In figure 2.16 [10], Ponta and Aref compare the vorticity field obtained by KLE at $A 1$ with a laser-fluorescene photograph, in the case of an oscillating cylinder at $R e=140, \lambda / D=6.07$, and $A / D=0.5$, courtesy Prof. Williamson in [10].

The non-dimensional vorticity field at point $A 1$ is shown in Figure 2.17. It shows the successive triplet structures as evolving downstream. Ponta and Aref have checked that adding up the circulation of two vortices with a positive sign is exactly opposite the circulation of the negative vortex, which yields to nearly zero circulation produced in one cycle. In close- 

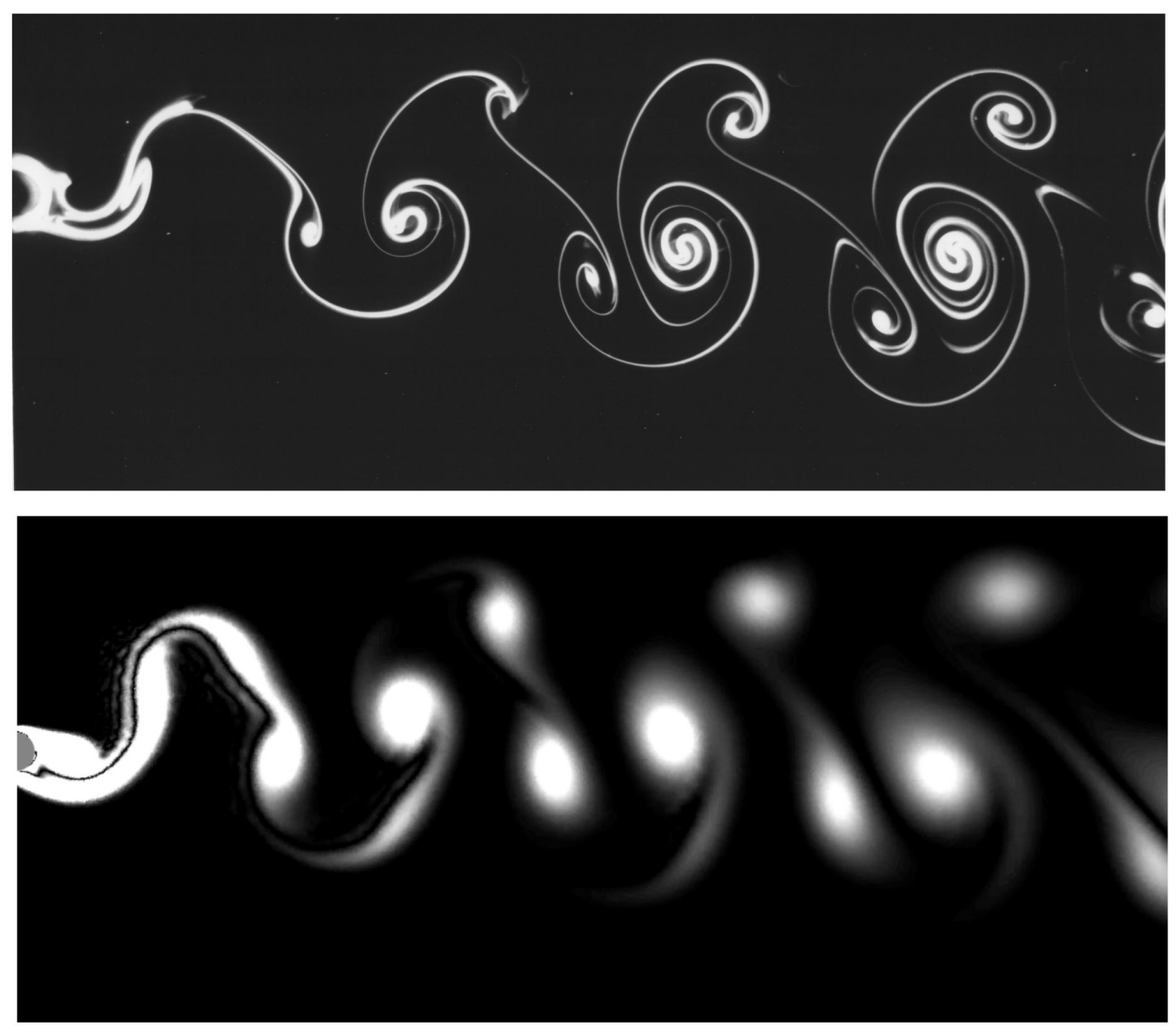

Figure 2.16. Vorticity field shown in gray scale as obtained by KLE at $R e=140$ compared with a flow visualization by C. H. K. Williamson(courtesy Prof. Williamson in [10], see permission in appendix), of a $\mathrm{P}+\mathrm{S}$ wake of an oscillating cylinder for the same Re, [Figure created by author from data in 10]. 


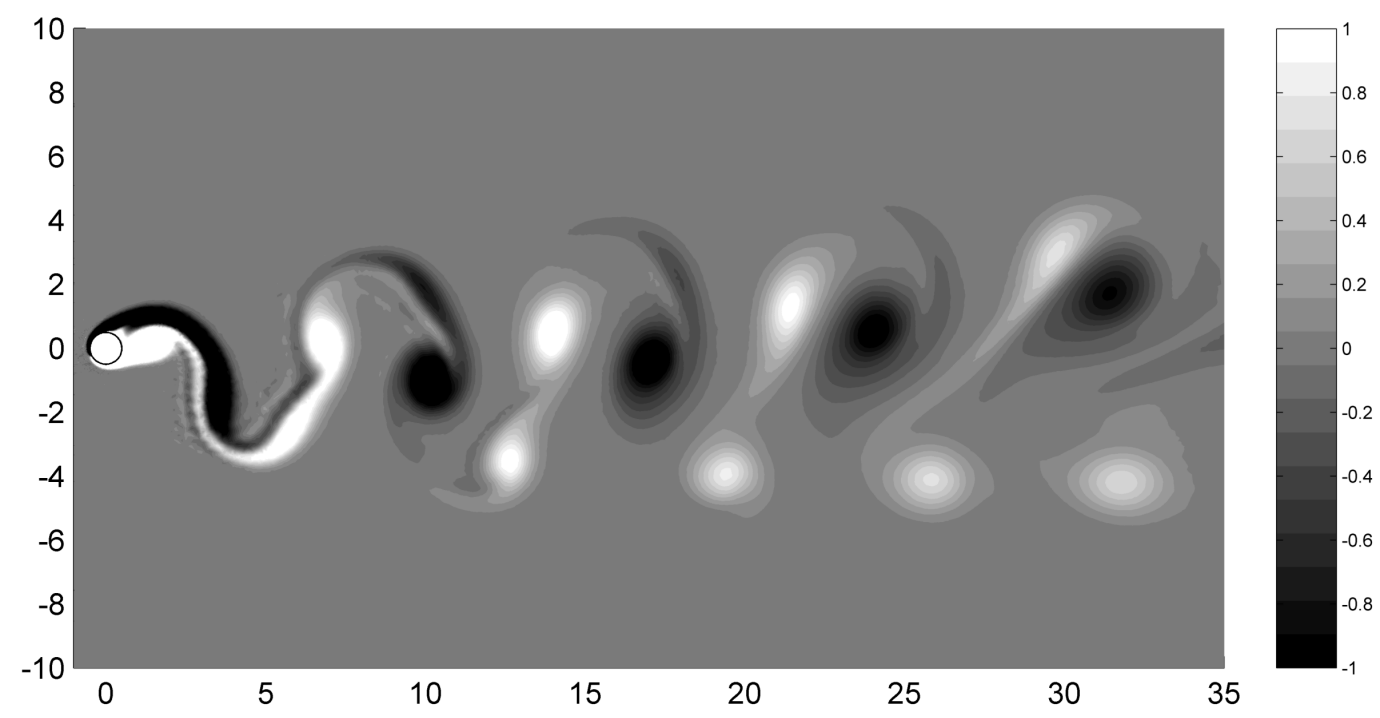

Figure 2.17. Non-dimensional vorticity field obtained by KLE for a $\mathrm{P}+\mathrm{S}$ wake with $R e=140$, $\lambda / D=7.5$, and $A / D=1$, [Figure created by author from data in 10].

up views of Figure 2.18, a sequence plot shows the non-dimensional vorticity field during the course of splitting giving rise to the $\mathrm{P}+\mathrm{S}$ pattern. It starts from the bottom-left panel and follows clockwise. It is seen that the lower positive half of the near wake stretches breaking away into two vortices due to the straining effect of the neighboring vortices. The upper negative half, however, does begin to split, different from its positive counterpart. Most of the vorticity that is shed stays together, forming a vortex strong enough to recover its tail [10]. It takes longer to undergo the last process and complete the sequence.

The empty circles in Figure $2.13(b)$ show a $\mathrm{P}+\mathrm{S}$ wake with a pattern not as strong as $A 1$. As an example, Figure 2.19 depicts a non-dimensional vorticity field representing a weak $\mathrm{P}+\mathrm{S}$ mode at point $B(\lambda / D=8.5, A / D=1)$. Downstream, the pattern remains $(\mathrm{P}+\mathrm{S})$, since the relatively weaker upper positive vortex remains independent of the stronger, lower, positive vortex. The third vortex, however, dissipates quickly downstream [10].

Point $C(\lambda / D=8.5, A / D=0.3)$ is in a region called 'no synchronized pattern observed', lying way off the $\mathrm{P}+\mathrm{S}$ region on the WR map. Its corresponding non-dimensional vorticity field is shown with its disorganized vortex patterns in Figure 2.20, that is confirmed by experimental observations. 

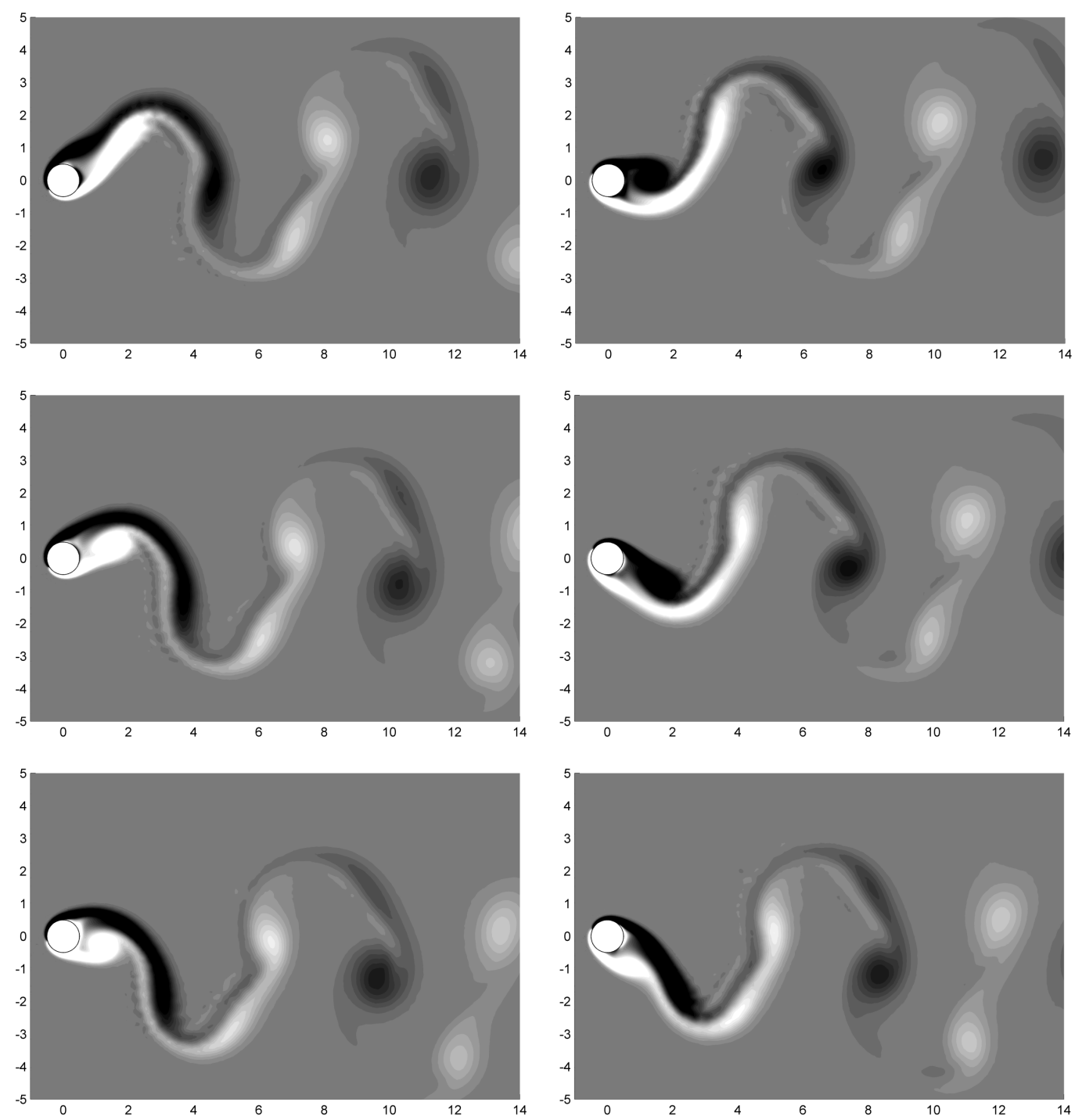

Figure 2.18. plot showing the sequence of events of the vortex splitting process producing a $\mathrm{P}+\mathrm{S}$ wake starting from the bottom left panel moving clockwise, [Figure created by author from data in 10]. 


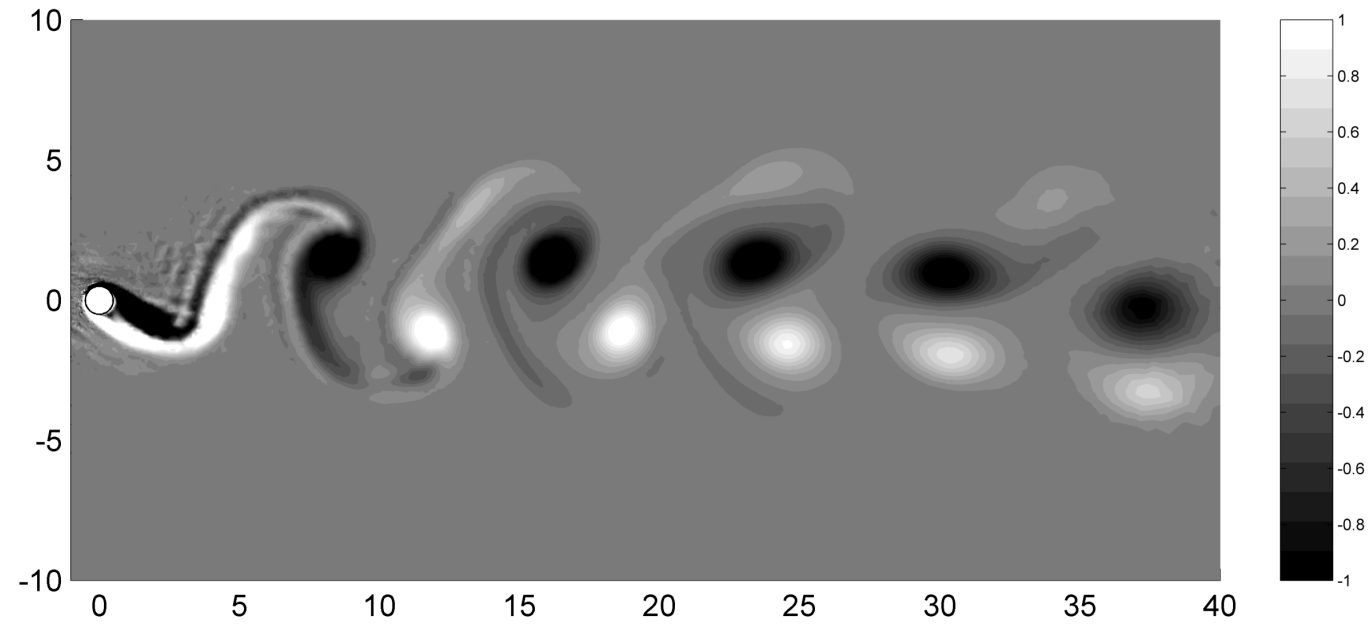

Figure 2.19. Non-dimensional vorticity field for a weak $\mathrm{P}+\mathrm{S}$ wake with $R e=140(\lambda / D=8.5$, $A / D=1)$, [Figure created by author from data in 10].

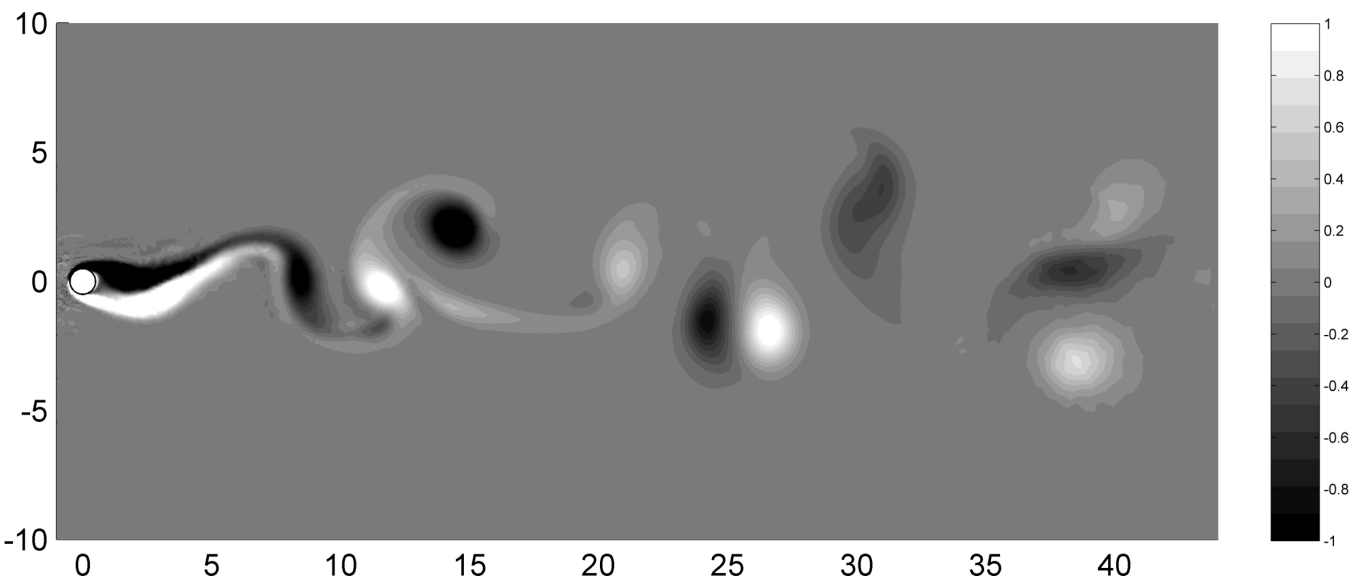

Figure 2.20. Non-dimensional vorticity field for a chaotic wake with $R e=140(\lambda / D=8.5$, $A / D=0.3)$, [Figure created by author from data in 10]. 


\subsubsection{Secondary vortex streets and complex wakes}

The generation of secondary vortex streets behind a bluff body was observed by numerous researchers, both experimentally and numerically [57-63]. Taneda [62] used flow visualization techniques to observe this phenomenon behind a stationary cylinder in a uniform stream. He saw how primary Kármán streets started decaying downstream, while the secondary one showed scaled up vortex formation with a frequency less than that for the primary street. Matsui and Okude [60] studied secondary vortex street formation using flow visualization along with hot-wire measurement techniques on acoustically-forced cylinder wakes and found that the secondary street is formed when the primary streets merge. Forcing the wake acoustically by a third or half the Kármán frequency led to a merging of every two or every three vortices. Cimbala et al. [58] and Williamson and Prasad [63] worked on secondary vortex street formation in unforced wakes. Aref and Siggia [57] and Meiburg [61], did a numerical study of the secondary vortex street using sub-harmonic perturbations applied to oppositely signed vortex blobs modelling the Kármán street. For both positive and negative blobs merging could be seen showing how important it is for secondary vortex streets to form. Inoue and Yamazaki [59] resorted to solving the Navier-Stokes numerically and saw a merging by pairing phenomena in the wake of stationary cylinders in a uniform stream on being perturbed by an in-line sinusoidal function. These cases with a forced wake are in some ways analogous to a combined motion consisting of a cylinder moving with a constant velocity with no component in the vertical direction and oscillating in-line. One should note that in a lot of cases involving merging some kind of application of an in-line fluctuation is present. In Ponta and Aref's [10] study, in-spite of a stronger vertical oscillation, the in-line oscillations (required to ensure that tangential velocity does not change) leads to merging. They suggested that vortices undergoing a packing-unpacking mechanism in the primary street could be because of the in-line fluctuation resulting in an unstable Kármán array and hence triggering merging. The vertical component of oscillation is also important, since it results in the primary wake frequency to be set by the vertically forced oscillation.

The processes of vortex interaction strongly depend on the kinematics of the body motion such as changes in the parameters of the trajectory as shown in Ponta and Aref [10]. The discussion was presented on how the pattern of vortices produced due to forced oscillations were formed, shed and then evolved for a cylinder for Reynolds number less than 180 (the approximate periodic laminar wake regime for Kármán street). The case of $R e=U D / \nu=$ 140 was studied in details since it represents the above mentioned range showing various vortex structure arrangements.

Figure 2.22 shows the vorticity plot for a wake behind a cylinder oscillating at the same frequency as in Figure. 2.17but following a trajectory that keeps the tangential speed constant. This relatively subtle change in trajectory, gives a totally different pattern: two separated 
vortex units per shedding cycle were produced in the near-wake, arranging as a closepacked Kármán vortex street. Then, the neighbouring vortices merge within either row, forming the so called hyper-Kármán vortex-street having a set of larger vortices with double the spacing and half the frequency of the original one. An extreme example of vortex merging is the so-called vortex coalescence. The coalescence is to arrange many smaller vortices that are formed by forced oscillations with higher frequencies into Kármán-like vortex street [11]. This also corresponds to very frequent interruptions of the shedding process due to the angle change in the inflow on the C-sectors. A lot of vorticity cumuli, after advecting downstream, merge into larger Kármán-like structures.

\section{The formation of a hyper-Kármán vortex-street}

Ponta and Aref [10] moved the cylinder at constant relative speed to the fluid to decrease the influence of the cylinders fluctuations in velocity. Tangential velocity of the cylinder $U_{t g}$ is expressed as follows:

$$
U_{t g}=\sqrt{V_{x, c y}^{2}+V_{y, c y}^{2}} .
$$

If the vertical velocity is kept the same to maintain a constant period of forced oscillation, the velocity component in the horizontal direction is adjusted for maintaining $U_{t g}$ constant and equal to the free stream speed $U$ by,

$$
V_{x, c y}=U \sqrt{1+\left(2 \pi \frac{A}{\lambda} \cos \left(2 \pi \frac{t U}{\lambda}\right)\right)^{2}} .
$$

Ponta and Aref [10] studied this phenomenon by using a constant-speed path at point $A 1$ $(\lambda / D=7.5, A / D=1)$ as shown in Figure 2.21. One cycle of this path is shown in Panel (a) in which geometrical coordinates are based on the diameters. The solid line shows the path following the cylinder's centre while the dotted circles indicate its circumference. For point $A 1(\lambda / D=7.5, A / D=1)$, the average horizontal velocity $U_{x, m}$ equals 0.789 of the free stream velocity $U$. Panel $(b)$ illustrates this path of the cylinder corresponding to a frame of reference moving in the horizontal direction with constant velocity $U_{x, m}$.

Figure 2.22 depicts the non-dimensional vorticity field in the case of constant velocity mentioned above. As shown, it produces the wake with two single vortices in each shedding cycle, with a closely packed, well defined Kármán vortex street arrangement. The wake goes on for approximately 10 diameters downstream and, then produces a perfectly-symmetric 

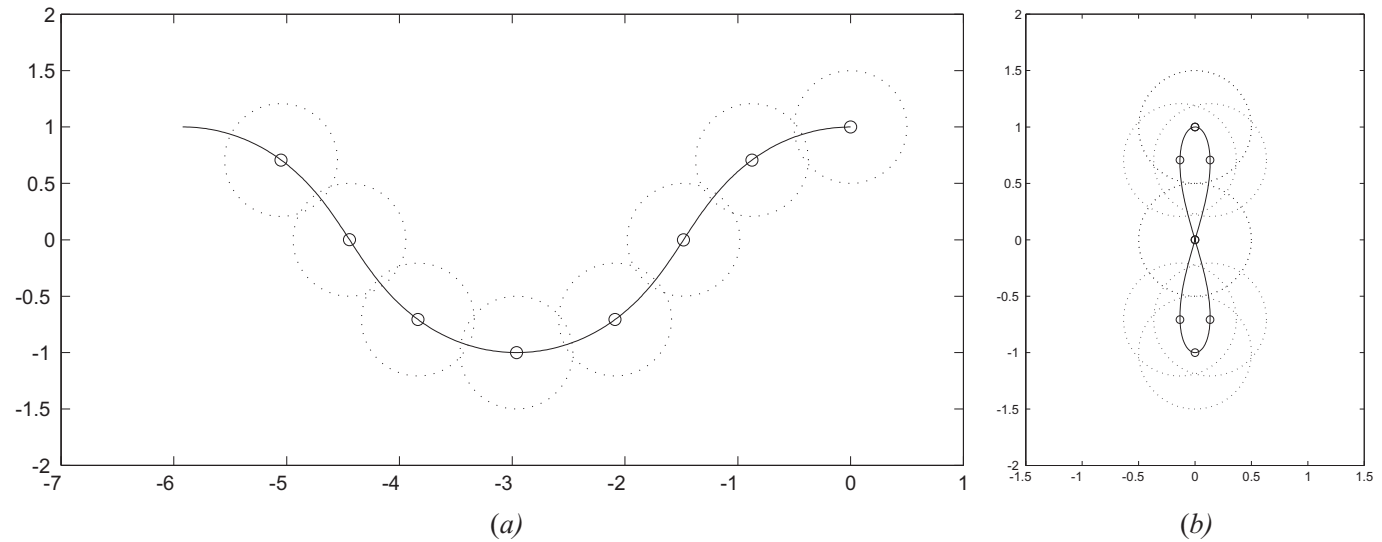

Figure 2.21. (a), Path followed by the cylinder's center at a constant speed for $A 1(\lambda / D=7.5$, $A / D=1)$. (b), Path of the cylinder when the reference frame moves horizontally with speed $U_{x m}$. [Figure created by author from data in 10] 


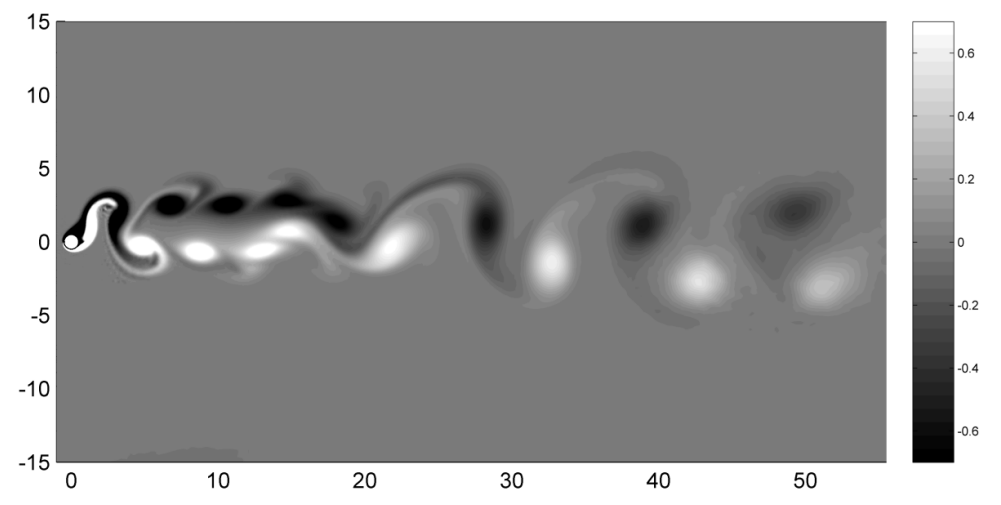

Figure 2.22. Non-dimensional vorticity field obtained by KLE for the hyper-Kármán vortex-street with $R e=140, \lambda / D=7.5$, and $A / D=1$, [Figure created by author from data in 10].

and more regular vortex street. Compared to what we have seen in the formation of $\mathrm{P}+\mathrm{S}$ and $2 \mathrm{P}$ secondary wake, the change in the trajectory has made the asymmetric effect in the splitting process disappeared. Thus, it provides a strong proof to Ponta and Aref [10]'s hypothesis that the role of the fluctuations in velocity is key to vortex splitting, affecting the primary wake's symmetry.

The sequence of plots in Figure 2.23 shows the non-dimensional vorticity field at the time of merging of vortices of the hyper-Kármán vortex-street. It starts from the bottom-left panel moving clockwise. Two cycles of the oscillation produces a single completed loop. The figure illustrates that two separate vortex units are produced in the near wake each shedding cycle, that are arranged as a close-packed Kármán vortex street. This is then absorbed by the subsequent pair giving rise to larger structures reducing the forced oscillation frequency by half.

For the trajectory where the streamwise velocity is constant, Ponta and Aref used $U$ as a reference speed for Reynolds number as well as geometrical wavelength $\lambda$. The forced oscillation in this case had a period $\lambda=T_{e} U$. Therefore, the parameter $\lambda / D$ can be interpreted as a Non-dimensional geometrical wavelength or as a non-dimensional period of forced oscillation $\left(\lambda / D=T_{e} U / D\right)$. However, for the path with constant-velocity they used $U_{t g}$ for $R e$ and the Non-dimensional period of the forced oscillation. Now the geometrical wavelength has to be interpreted in terms of the average horizontal velocity $\left(\lambda_{g}=T_{e} U_{x m}\right)$. Now the parameter $\lambda / D$ can be interpreted in different ways. For instance, if $\lambda / D$ is interpreted as a non-dimensional period of the forced oscillation, the above case stays at $A 1$. But, if interpreted as a non-dimensional geometrical wavelength, they shift to $A 2$ in the WR map (empty square in figure 2.13 $(b)$ ). 

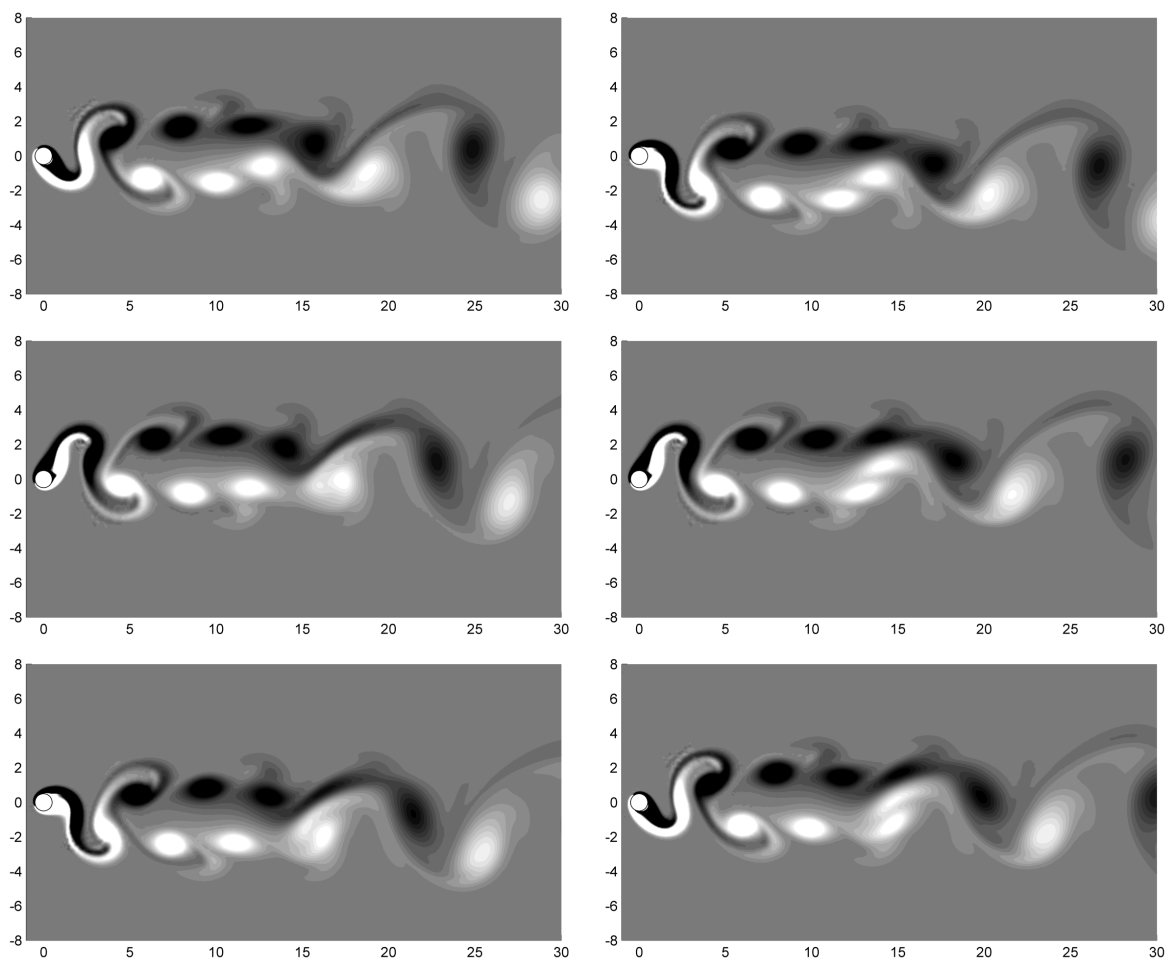

Figure 2.23. plot depicting the vortex-merging mechanism producing the hyper-Kármán vortexstreet, [Figure created by author from data in 10]. The sequence starts at the bottom left panel moving clockwise. 


\section{NUMERICAL SOLUTION OF UNSTEADY-FLOW PROBLEMS}

\subsection{Introduction of the Navier-Stokes equations}

In a mathematical sense, Navier-Stokes equations are a system of $2^{\text {nd }}$ order non-linear Partial Differential Equations. These consist of three basic conservation equations:

1. Conservation of mass or the continuity equation

$$
\frac{\partial \rho}{\partial t}+\nabla \cdot(\rho \mathbf{v})=0
$$

where $\rho$ is density of the fluid, $\mathbf{v}$ is velocity field and $t$ is time.

2. Conservation of momentum

$\rho \frac{\mathrm{d} \mathbf{v}}{\mathrm{d} t}=\rho \mathbf{g}-\nabla P+\nabla \cdot \boldsymbol{\sigma}$

where, $P$ is pressure, $\boldsymbol{\sigma}$ is stress tensor, and $\rho \mathbf{g}$ is body force.

3. Conservation of energy

$$
\rho \frac{\mathrm{d} \hat{U}}{\mathrm{~d} t}+p(\nabla \cdot \mathbf{v})=\nabla \cdot(k \nabla T)+\mathbf{\Phi}
$$

where $\hat{U}$ is the internal energy per unit mass, $\mathrm{k}$ is the thermal conductivity, $\nu$ is the kinematic viscosity and the function $\Phi$ represents energy dissipated due to viscous effects.

This set of equations may completely define fluid motion allowing us to solve for various flow quantities like pressure, velocity, temperature, and density. In the more general case of the incompressible flow of Newtonian fluids, the density is considered constant and the above equations reduce to 
1. Conservation of mass or the continuity equation

$\nabla \cdot \mathbf{v}=0$

2. Conservation of momentum

$\rho \frac{\mathrm{d} \mathbf{v}}{\mathrm{d} t}=\rho g-\nabla P+\mu \nabla^{2} \mathbf{v}$

where $\mu$ is the coefficient of dynamic viscosity.

3. Conservation of energy

$\rho C_{v} \frac{\mathrm{d} T}{\mathrm{~d} t}=k \nabla^{2} T+\Phi$

where $C_{v}$ is specific heat at constant volume.

\subsection{Numerical Solution of the incompressible viscous flow}

The following section deals with some of the more popular formulations of the NavierStokes equations that models a homogeneous, viscous incompressible fluid flow in an inertial frame of reference, along with some discussion on the issues of boundary conditions. As mentioned before, for a homogeneous incompressible flow, the density remains constant giving us a set of equations (Eq 3.1-3.3). These equations clearly imply the decoupling of the momentum equation with the energy equation for the incompressible flow. The most obvious advantage of this decoupling is that the momentum equation and the continuity equation can now be solved independent of temperature to obtain the required velocity and pressure field. This decoupling might seem like it makes things easier, but that is not the whole picture, as will become clear in the subsequent discussion.

The first step in solving the Navier-Stokes equations in the numerical sense would be to decide upon the set of variables representing the equations followed by a group of boundary conditions. As known, the Navier-Stokes equations fall into two main categories of the formulations: the primitive variable formulations and the non-primitive variable formulations. Both possess their own set of advantages and disadvantages, which shall be discussed in brief, but in both cases the major problem and the oldest point of contention are the boundary conditions. It is mostly the pressure and vorticity which pose a problem since they do not have what can be called an obvious physical representation at the boundaries.

\subsubsection{Primitive variables}

For incompressible viscous fluid, the most fundamental formulation of the Navier-Stokes equations would be based on the pressure-velocity formulations. These are what are called 
the primitive variable formulations. The incompressible Navier-Stokes equations in the form of the so-called primitive variables is written as

$$
\frac{\partial \mathbf{v}}{\partial t}+v \cdot \nabla \mathbf{v}=-\nabla p+\nu \nabla^{2} \mathbf{v}+\mathbf{g}
$$

where $p=P / \rho$, defined on a spatial domain $\Omega$ with the boundary $S$, is the pressure divided by the constant fluid density; $\nu$ is the kinematic viscosity, and $\mathbf{v}$ is the velocity field. The Navier-Stokes equations represented by the primitive variables can be called a mixed elliptic parabolic equation being parabolic in time mainly because of the convective diffusive term and elliptic in space due to the interaction between pressure and the conservation of mass. To define this aforementioned problem completely, the equation needs to be supplemented by a set of boundary conditions specifying it as an initial value boundary value (IVBV) problem. The most common approach would be to start with the specification of an initial value for $\mathbf{v}$ on $\Omega$

$$
\mathbf{v}\left(x, t_{0}\right)=\mathbf{v}_{0}(x) \text {, such that } \boldsymbol{\nabla} \cdot \mathbf{v}_{0}=0, \text { where } x \in \Omega
$$

followed by specifying the velocity at the boundary

$$
\mathbf{v}(x, t)=\mathbf{v}_{S}(x, t), x \in S=\mathbf{b}
$$

along with a global continuity condition obtained by volume integration of the continuity equation and then using the Gauss theorem

$$
\oint_{S} \mathbf{n} \cdot \mathbf{b d} \mathbf{S}=\mathbf{0}
$$

where, $\mathbf{n}$ is a normal unit vector to the surface of the boundary. So in this simplest of representations there is indeed no boundary condition for pressure, giving rise to a series of problems which are still a topic of debate.

\section{Solution strategies}

As discussed above, understanding pressure is the main difficulty to solve the incompressible Navier-Stokes equations numerically. Unlike velocity, pressure does not have any explicit representation. There is no evolutionary pressure equation. The main reason is that since we have assumed an incompressible condition, pressure now loses its thermodynamic meaning and serves only as some kind of a Lagrange operator to ensure the incompressibility condition. Since the pressure term is of elliptic character, the solution anywhere would be influenced by the solution at every point in the domain. Physically this means that the pressure waves travel at infinite velocity to all points in the domain for every time step. In other words, the pressure has to adjust instantaneously throughout the domain to account for any disturbance in order to maintain zero divergence of velocity. This leads to the intricate coupling between the two variables. This statement is the driving factor for 
non-fractional approach or, in other words, the standard discretization approach. Thus a numerical scheme with proper boundary conditions must be devised which can take into consideration the implicit coupling between pressure and velocity and keeps the flow incompressible throughout the domain. At the same time, the pressure and velocity terms can be decoupled in this method and velocity and solve for each separately to avoid having to tackle the unwieldy simultaneous equations.

It is, in fact, possible to solve this set of equations simultaneously as a coupled system [64]. This is rarely seen in finite difference methods [64] due to the complex matrices obtained as opposed to a regular block diagonal matrix. Though this method is more common in finite element analysis, it does have its own set of difficulties, as mentioned by Glowinski [65] arising from :

1. The equations are non-linear.

2. The continuity equation.

3. The coupling between the set of equations owing to the advection term and continuity equation.

The divergence of the momentum equation obtained under the continuity condition gives Poisson's pressure equation, which is thought to be the most common approach to getting rid of the continuity equation. This, along with other details, will be discussed in the subsequent section.

The two main approaches in a pressure-velocity formulation are the fractional step and the non-fractional step methods.

\subsubsection{Non-fractional step methods}

The major difference between the two approaches is that in non-fractional step methods the velocity and pressure evolve simultaneously, whereas in the fractional step method the convection part is treated separately from the pressure or incompressibility part. Eq 3.4 and $\mathrm{Eq} 3.5 \mathrm{can}$ be linearized by discretizing in time and neglecting the body forces to give

$$
\begin{gathered}
\frac{\mathbf{v}^{n+1}-\mathbf{v}^{n}}{\Delta t}+\nabla p^{n+1}=\nu \nabla^{2} \mathbf{v}^{n+1}-\mathbf{v}^{n} \cdot \boldsymbol{\nabla} \mathbf{v}^{n} \\
\boldsymbol{\nabla} \cdot \mathbf{v}^{n+1}=0
\end{gathered}
$$


where, $\mathbf{v}$ is the velocity vector and $p$ is the pressure term

Rewrite Eq 3.9, it gives

$$
[-\Delta+\gamma \mathbf{I}] \mathbf{v}^{n+1}+\nabla p^{n+1}=g\left(\mathbf{v}^{n}\right)
$$

where, $\nu$ is the kinematic viscosity, $\mathbf{I}$ is an Identity matrix, $\gamma=\frac{1}{\nu \Delta t}$ and $g\left(\mathbf{v}^{n}\right)=\gamma \mathbf{v}^{n}-$ $\nu^{-1} \mathbf{v}^{n} \cdot \nabla \mathbf{v}^{n}$

Here, a two-level scheme has been adopted, treating the pressure and viscous term implicitly and the non-linear advection term explicitly in time. There could be other methods in time discretization such as a fully implicit scheme or even a semi-implicit scheme using linearization techniques. It is widely accepted to use a higher order explicit scheme like the Adams-Bashforth scheme for the non-linear advection term and a Crank-Nicolson scheme for the linear terms. This can be followed by linearizing the advection term using a point iteration scheme. There are many other higher order and more accurate schemes, but the one mentioned in the above equations is just for explanatory purposes. As previously mentioned, a Poisson's Equation is obtained from the divergence of the momentum equation under the continuity condition. This gives us the following set of equations :

$$
\begin{gathered}
{[-\Delta+\gamma \mathbf{I}] \nabla \cdot \mathbf{v}^{n+1}+\nabla p^{n+1}=g\left(\mathbf{v}^{n}\right)} \\
\nabla^{2} p^{n+1}=\boldsymbol{\nabla} \cdot g\left(\mathbf{v}^{n}\right)
\end{gathered}
$$

Even though the above relation is obtained by the continuity equation, its solution does not imply the incompressibility condition, all it shows is that $[-\Delta+\gamma \mathbf{I}] \nabla \cdot \mathbf{v}^{n+1}=0$. Therefore it simply tells us that $\boldsymbol{\nabla} \cdot \mathbf{v}^{n+1}$ is harmonic but not necessarily zero. However, Kleiser and Schumann [66] showed that if divergence at the boundary is forced to zero

$$
\left.\nabla \cdot \mathbf{v}^{n+1}\right|_{S}=0
$$

incompressibility should be ensured throughout the domain. This follows from the mean value theorem/ extremum theorems of harmonic functions which means if some harmonic function equals a constant at the surface completely enclosing a domain, its value will be equal to that constant throughout the domain. This leads us to a BVP with two elliptic equations

$$
\begin{array}{r}
{[-\Delta+\gamma \mathbf{I}] \boldsymbol{\nabla} \cdot \mathbf{v}^{n+1}+\boldsymbol{\nabla} p^{n+1}=g\left(\mathbf{v}^{n}\right)} \\
\nabla^{2} p^{n+1}=\boldsymbol{\nabla} \cdot g\left(\mathbf{v}^{n}\right) \\
\left.\mathbf{v}^{n+1}\right|_{S}=\mathbf{b}^{n+1} \\
\left.\boldsymbol{\nabla} \cdot \mathbf{v}^{n+1}\right|_{S}=0
\end{array}
$$

along with the global constraint Eq 3.8 i.e. $\oint_{S} \mathbf{n} \cdot \mathbf{v}_{\mathbf{S}} \mathbf{d} \mathbf{S}=\mathbf{0}$. Here there are two boundary conditions for $\mathbf{v}$ and none for pressure, which means that the above equations still have to be solved simultaneously. So, it is imperative to look for some method to decouple 
the two equations by coming up with a legitimate boundary condition for pressure. As mentioned in Quartapelle and Napolitano [67], Glowinksi and Pironneau devised a finite element method based on an additional equation for the scalar velocity potential. Another method was based on the influence matrix technique proposed by Kleiser and Schumann [67]. Both these methods propose to implement the pressure boundary conditions using an additional linear problem. This was followed by Quartapelle and Napolitano's paper on implementing integral Boundary conditions on pressure [67] which seemed to provide a better physical interpretation owing to the elliptic nature of the Poisson's equation for pressure.

\subsubsection{Fractional step methods}

This method, first introduced by Chorin and Tenman, is one of the most widely used methods for solving the Navier-Stokes equations of the primitive variable. Consider the Navier-Stokes equations in the following form, with the body forces neglected:

$$
\begin{array}{r}
\frac{\partial \mathbf{v}}{\partial t}+v \cdot \nabla \mathbf{v}=-\nabla p+\nu \nabla^{2} \mathbf{v} \\
\boldsymbol{\nabla} \cdot \mathbf{v}=\left.0 \quad \mathbf{v}\right|_{S}=\mathbf{b}
\end{array}
$$

The general procedure is to advance the velocity in time and approximate an intermediate velocity from the first equation, without considering the pressure term. As already mentioned, the pressure term in the above equation acts as a Lagrange multiplier to enforce incompressibilty at every time-step, therefore, the calculated velocity will not be divergence free. To obtain a velocity of divergence free, the calculated velocity is then used to solve an elliptic equation by enforcing the incompressibilty condition. Subsequently a pressure field is determined for that time step. In the intermediate step, the following equation is obtained by avoiding completely the pressure term

$$
\frac{\mathbf{v}^{*}-\mathbf{v}^{n}}{\triangle t}=-\left(\mathbf{v}^{n} \cdot \boldsymbol{\nabla}\right) \mathbf{v}^{n}+\left.\nu \nabla^{2} \mathbf{v}^{n} \quad \mathbf{v}^{*}\right|_{S}=\mathbf{b}^{n+1}
$$

Obviously, as stated above, the velocity field $\mathrm{v}^{*}$ would not be divergence free, which leads to:

$$
\begin{gathered}
\frac{\mathbf{v}^{n+1}-\mathbf{v}^{*}}{\Delta t}=-\nabla p^{n+1} \\
\nabla \cdot v^{n+1}=0
\end{gathered}
$$




$$
\left.\mathbf{n} \cdot \mathbf{v}^{\mathbf{n}+\mathbf{1}}\right|_{\mathbf{s}}=\mathbf{n} \cdot \mathbf{b}^{\mathbf{n}+\mathbf{1}}
$$

Equation 3.18 can also be formulated as

$$
\mathbf{v}^{*}=\mathbf{v}^{n+1}+\Delta t \nabla p^{n+1}
$$

where, $\nabla p^{n+1}$ is not the gradient of pressure but of some artificial scalar function proportional to the unknown Pressure often referred to as the "Pseudo-pressure". The quantity $\mathbf{v}^{n+1}$ which is required the velocity field is actually the solenoidal component of $\mathbf{v}^{*}$ and not the real $\mathbf{v}^{n+1}$ as the tangential boundary condition is not necessarily met. The normal boundary value for velocity is a consequence of the above step being inviscid. So the required velocity is calculated through the projection of the velocity $\mathbf{v}^{*}$ onto a solenoidal space. The basis for the above step is the helmholtz-hodge decomposition of the velocity field (due to Ladyzhenskaya) which states that it is possible to decompose any vector field as $\mathbf{V}=\mathbf{W}+\boldsymbol{\nabla} \phi$ where, $\mathbf{W}$ is solenoidal and $\mathbf{n} \cdot \mathbf{W}=\mathbf{0}$ and $\phi$ is the potential function with its gradient giving the irrotational component of $\mathbf{V}$. The gradient term can be further decomposed into $\nabla \phi=\nabla \phi_{0}+\nabla h$, where $h$ is a harmonic function and $\left.\phi_{0}\right|_{S}=0$. This leads to the vector $\mathbf{V}$ being decomposed into

$$
\mathbf{V}=\mathbf{W}+\nabla \phi_{0}+\nabla h
$$

Using this decompostion for the divergent $\mathbf{v}^{*}$

$$
\mathbf{v}^{*}=\mathbf{W}+\nabla \phi_{0}+\nabla h
$$

Adding and subtracting another harmonic function $h_{B}$ to the RHS of Eq 3.23

$$
\left.\mathbf{v}^{*}=\left[\mathbf{W}+\nabla h_{B}\right]+\left[\boldsymbol{\nabla}\left(h-h_{B}\right)\right]+\nabla \phi_{0}\right] \quad \mathbf{n} \cdot \boldsymbol{\nabla} \mathbf{h}_{\mathbf{B}}=\mathbf{n} \cdot \mathbf{b}
$$

Clearly $\left[\mathbf{W}+\nabla h_{B}\right]$ represents $\mathbf{v}^{n+1}$ for an incompressible flow not satisfying the no-slip condition for the second half step. Thus, $\mathbf{v}^{n+1}$ can be found using a projection of $\mathbf{v}^{*}$ on the divergence free space.

In order to calculate the velocity from $\mathrm{Eq} 3.18$ and $\mathrm{Eq} 3.20$, the divergence of $\mathrm{Eq} 3.18$ is substituted into Eq 3.19 to get the Poisson's Equation for pressure also called the PPE

$$
-\nabla^{2} p^{n+1}=\frac{-1}{\triangle t} \nabla \cdot \mathbf{v}^{*}
$$

Using Eq 3.20 along with the boundary condition $\left.\mathbf{v}^{*}\right|_{S}=\mathbf{b}^{n+1}$ the following boundary condition for the PPE can be derived

$$
\left.\mathbf{n} \cdot \nabla \mathrm{p}^{\mathrm{n}+1}\right|_{\mathrm{s}}=\mathbf{0}
$$

As soon as the pressure field is determined, the required velocity field can be determined solving Eq 3.18 and Eq 3.20, The basic disadvantage here as mentioned before is that the second half step ensuring the incompressibility condition is inviscid, thereby, able to ensure only the normal component of the velocity boundary condition. This error is slightly qual- 
ified because the velocity boundary condition in the first half step is the no-slip condition. There are methods that introduce the viscous component in the second half step. One such method is mentioned in Quartapelle [66] in the form of a Crank-Nicholson scheme

$$
\begin{array}{ll}
\frac{\mathbf{v}^{*}-\mathbf{v}^{n+1}}{\triangle t}=-\left(\mathbf{v}^{n} \cdot \boldsymbol{\nabla}\right) \mathbf{v}^{n}+\frac{1}{2} \nu \nabla^{2} \mathbf{v}^{n} & \left.\mathbf{v}^{*}\right|_{S}=\mathbf{b}^{n+1} \\
\frac{\mathbf{v}^{n+1}-\mathbf{v}^{*}}{\triangle t}=-\nabla p^{n+1}+\frac{1}{2} \nu \nabla^{2} \mathbf{v}^{n+1} & \left.\mathbf{v}^{n+1}\right|_{S}=\mathbf{b}^{n+1}
\end{array}
$$

The viscous term has second order accuracy but, like the non-fractional step schemes, some complicated pressure condition must be used to enforce the incompressibility.

\subsubsection{Artificial incompressibility}

This method, first proposed by Chorin in 1967, allows analysts to take advantage of the immense advances made in the analysis of compressible flow. The key of this method is use a slightly modified version of the continuity equation in order to make it compressible and, hence, solve it as an evolution equation in pressure. In order to achieve that, a time derivative of the pressure term is added to the continuity equation giving

$$
\frac{1}{\beta} \frac{\partial p}{\partial t}+\frac{\partial \mathbf{v}_{i}}{\partial \mathbf{x}_{i}}
$$

This was originally introduced for the steady state Navier-Stokes equations, so that, when the steady state is achieved, the artificial compressibility term vanishes. Here " $t$ " does not represent the real physical time but an artificial "pseudo" time. This gives a mixed hyperbolic/parabolic form of equations, and many algorithms developed for similar compressible flows could be used to solve these equations. For a steady state formulation Chorin proposed a leap-frog time differencing scheme for pressure and a Dufort-Frankel space differencing method for velocity at the regular grid points. Peyret and Taylor adopted the staggered grid formulation explicit in time [68]. What this method implies on a physical level is that pressure waves now travel at a finite speed depending on the incompressibility factor $\beta$ rather than instantaneously propagate to all points in the domain, thus the choice of $\beta$ is very important. The higher its value the closer the formulation will be to incompressible flow. But at the same time, however, too high a value will tend to make the equation stiff. But if $\beta$ is too small then the propagation speed will be too slow which will effect other factors like the viscous boundary layer, flow separation, etc which might prevent convergence.

The iteration of the governing equations in the "pseudo" time continues until steady state is achieved. Although because of the compressibility introduced in the continuity equation this method was not preferred for unsteady flow, it has been proven successful for such 
flows as well [69]. The general idea for unsteady flows would be to use an iterative procedure using an artificial compressibility method for each physical time step, ensuring that incompressibility is met at each step.

\subsubsection{Non-Primitive variables}

Given the importance of vorticity as a physical variable, especially in vortex dominated flows, a vorticity-based formulation of the Navier-Stokes equations is a popular alternative to the primitive variable formulation. In such cases, it makes more sense to analyze the flow based on vorticity, which has an extensively researched and understood transport equation. The study of vortex generation at boundaries, along with its diffusion and advection are very important in analyzing flow seperation, drag etc. in vortex dominated flows. Mathematically, owing to the vorticity $\boldsymbol{\omega}$ being one order higher than the velocity $\mathbf{v}$ a vorticity formulation implicitly gives a more accurate velocity field. Vorticity-based methods also give a better estimate of the skin friction since they are based more on the shearing process itself. For flows with high Reynold's number the vorticity seems to be concentrated in the wake region, thereby, greatly reducing the computational domain. However, this formulation is also plagued by its own set of problems. The kinematic problem for the vorticity-velocity relation is overdetermined as it has both the Dirichlet and Neumann boundary conditions prescribed, whereas the dynamic vorticity transport equation has no boundary conditions for vorticity. Also, there does not seem to be any physical boundary condition for the vorticity independant of the velocity boundary conditions.

The creation of vorticity can be owing to the no-slip boundary condition which causes a torque and hence an angular velocity being imparted on the packets of fluid. This vorticity creation at the boundary should be represented by the vorticity boundary conditions and has been the general reasoning used to tackle this issue. Lighthill, one of the pioneers in this approach, proposed a fractional step method starting with an arbitrary vorticity boundary condition [70]. This gives rise to a velocity field which does not satisfy either of the two velocity conditions. He then proposed adding a velocity potential to take care of the normal flow, thereby getting slip velocity at the boundary. This slip velocity was called the vortex sheet and said to represent the boundary vorticity. Chorin [70] proposed a similar approach using the Prandtl boundary layer approximation. He basically split the NavierStokes equations into a viscid and an inviscid part. The Euler equation is then solved to give a slip velocity at the boundary. To get rid of this, vortex sheets are introduced and the resulting vorticity field is then used to find the solution of the diffusion equation to get the correct vorticity field at the desired time step. This formulation does not seem to satisfy the no-slip boundary condition as well as the normal boundary condition for velocity simultaneously and independent of the geometry. 
There have been many such models based on generation of vorticity at the boundary, but there is an entirely different school of thought which believes that a vorticity creation method does not fully explain vorticity interaction with solid boundaries. This led Quartapelle and Valgriz [66] to introduce an integral constraint on the vorticity. This "non-local" approach couples the vorticity everywhere in the domain to the boundary velocity. A similar approach was adopted by Anderson [70] who suggested requiring the time derivative of these integral constraints be made to vanish. These methods as per Quartapelle [66] are the true representation of the vorticity diffusion and interaction with solid walls. These are of course a kind of projection methods, where an initial "wrong" vorticity, based on an arbitrary vorticity boundary value is "corrected" by projecting it onto the space of harmonic functions. This is achieved by the integral condition. This Chapter deals with a particular type of formulation called the non-primitive variable formulation and a brief overview of solution strategies based on Quartapelle's book [66] is given for both two and three dimensional flows. The next Chapter covers hybrid formulation, also called the vorticity-velocity formulation.

\subsubsection{The Vorticity-Stream Function formulation for 2-D flows}

One possible way to get around the problem of pressure boundary conditions is to eliminate the pressure term entirely. This is exactly what is achieved in the Vorticity-Stream Function formulation of the Navier-Stokes equations. In this version, the Navier-Stokes equations are represented in terms of the vorticity $\boldsymbol{\omega}$ and the stream function $\psi$. So now the unknowns are $\boldsymbol{\omega}$ and $\boldsymbol{\psi}$ instead of $u, v, p$, reducing the number of unknowns by one. It also presents the added advantage of automatically taking care of the incompressibility condition owing to a property of the stream function. In two dimensions the above representation comprises two scalar equations obtained as follows:

In two dimensions vorticity $\boldsymbol{\omega}$ is a scalar given by

$$
\omega=\nabla \times \mathbf{v} \cdot \mathbf{k}
$$

while the velocity is represented as the curl of a Stream-function $\psi$ given by

$$
\mathbf{v}=\nabla \times \psi
$$

Equation 3.31 clearly implies that $\boldsymbol{\nabla} \cdot \mathbf{v}=0$. Substituting Eq 3.31 in Eq 3.30 gives the Poisson's equation for the Stream-function

$$
-\nabla^{2} \psi=\omega
$$

Taking the curl of the momentum equation and using Eq 3.30, Eq 3.31 and $\boldsymbol{\nabla} \cdot \mathbf{v}=0$ gives the vorticity transport equation

$$
\frac{\partial \omega}{\partial t}+J(\omega, \psi)=\nu \nabla^{2} \omega
$$

where, $J(\omega, \psi)$ is the Jacobian matrix representing the curl of the advection term namely, $\nabla \times[(\mathbf{v} \cdot \nabla) \mathbf{v}]$. 
The Dirichlet and Neumann conditions for the above two equations are derived conditions deduced by separately tackling the normal and tangential components of boundary conditions of the velocity Eq 3.7i.e. $\left.\mathbf{v}\right|_{S}=\mathbf{b}$. They are given by, $\left.\psi\right|_{S}=a$ and $\left.\frac{\partial \psi}{\partial n}\right|_{S}=b$,

where, $a=\int_{s_{1}}^{s} \mathbf{n} \cdot \mathbf{b d S}$ and $b=-\boldsymbol{\tau} \cdot \mathbf{b}$ given that $s_{1}$ is any fixed point on the boundary and $\tau$ is a unit vector tangential to the boundary. An initial condition for the vorticity can also be derived using the definition of vorticity and the initial condition for velocity Eq 3.6 giving the following initial condition

$$
\left.\omega\right|_{t=0}=\left(\nabla \times \mathbf{v}_{0}\right) \cdot \mathbf{k}
$$

Therefore the Navier-Stokes equations in the 2-D Vorticity-Stream Function formulation can be written as

$$
\begin{array}{r}
\frac{\partial \omega}{\partial t}+J(\omega, \psi)-\nu \nabla^{2} \omega=0 \\
-\nabla^{2} \psi=\omega \\
\left.\psi\right|_{s}=a,\left.\quad \frac{\partial \psi}{\partial n}\right|_{s}=b \\
\left.\omega\right|_{t=0}=\left(\nabla \times \mathbf{v}_{0}\right) \cdot \mathbf{k}
\end{array}
$$

Provided that

$$
\begin{array}{r}
\boldsymbol{\nabla} \cdot \mathbf{v}_{0}=0 \\
\frac{\partial a(S, 0)}{\partial s}=\mathbf{n} \cdot \mathbf{v}_{\mathbf{0}}
\end{array}
$$

One of the problems with this kind of formulation is the nonlinear advection term which also couples the vorticity and stream function variables. The other important issue is the overspecification of $\psi$ owing to both Dirichlet and Neumann conditions present as opposed to the underdetermined problem for $\omega$ with no boundary condition specified for it. The nonlinear terms can be dealt with using the standard linearizing techniques for non-linear equations. Some of the ways in which the problem of the boundary conditions is tackled are discussed below.

\subsubsection{Biharmonic formulation}

One way to avoid the boundary value problem for vorticity is to eliminate the vorticity term from the transport equation. This is achieved by substituting the Poisson's equation for the Stream Function into the vorticity transport equation resulting in the following equation

$$
\begin{array}{r}
\frac{\partial^{2} \nabla^{2} \psi}{\partial t}-\nu \nabla^{4} \psi+J\left(\nabla^{2} \psi, \psi\right)=0 \\
\left.\psi\right|_{S}=a \quad,\left.\quad \frac{\partial \psi}{\partial n}\right|_{S}=b
\end{array}
$$


where, $\psi_{0}$ is the solution of the Dirichlet problem

$$
-\nabla^{2} \psi_{0}=\left(\boldsymbol{\nabla} \times \mathbf{v}_{0}\right) \cdot \mathbf{k},\left.\quad \psi_{0}\right|_{S}=a(S, t)
$$

Where $\mathbf{v}_{0}$ and $a$ as before satisfy the solenoidal and the compatibility condition and $\mathbf{k}$ is the unit vector in the $\mathrm{Z}$ direction. Since the equation is fourth order elliptic, the specification of both dirichlet and Neumann conditions no longer make it overspecified.

\subsubsection{Coupled formulation in Vorticity and Stream Function}

Another method to eliminate problems associated with the overdetermined problem is to solve Eq 3.36 as a coupled equation in Vorticity and Stream Function even in the absence of the non-linear term. This is achieved by a unique coupling through the boundary conditions by associating one boundary condition with the transport equation and the other with the Poisson's equation. This can be written as

$$
\begin{array}{r}
\frac{\partial^{2} \nabla^{2} \psi}{\partial t}-\nu \nabla^{4} \psi+J\left(\nabla^{2} \psi, \psi\right)=0 \quad \text { such that }\left.\psi\right|_{S}=a \\
-\nabla^{2} \psi=\omega \quad \text { such that }\left.\frac{\partial \psi}{\partial n}\right|_{S}=b
\end{array}
$$

The spatial discretization for both these methods can be done by any of the three methods, namely, finite elements, finite differences, or spectral methods. Inspite of no direct implementation of the integral constraint, the couple formulation still satisfies the nonlocal character of vorticity. Hence further fortifying the idea of an integral condition on vorticity.

\subsubsection{Uncoupled formulation using vorticity integral conditions}

To split the two terms in the Vorticity-Stream Function formulation, it becomes necessary to determine supplementary conditions for vorticity to account for its lack of boundary conditions. Quartapelle and Valz-Cris [66] came up with an integral constraint on vorticity

$$
\int \omega \eta d \Omega=\oint\left(a \frac{\partial \eta}{\partial n}-b \eta\right) d S
$$

giving the following set of linearized equations

$$
\begin{array}{r}
(-\Delta+\gamma) \omega=f, \quad \int \omega \eta d \Omega=\oint\left(a \frac{\partial \eta}{\partial n}-b \eta\right) d S \\
-\nabla^{2} \psi=\omega,\left.\quad \psi\right|_{S}=a
\end{array}
$$

Where $\eta$ is any harmonic function defined in the domain $\Omega$. This is a semi-implicit discretization in time with $\omega \equiv \omega_{n+1}$ and $\psi \equiv \psi^{n+1}$ at the new time step $t^{n+1}$. One of the ways to implement the integral conditions and solve the uncoupled equations is based on

utilizing the linearity of the above formulation. It consists of decomposing the vorticity transport equation using the principle of superposition. The split formulation can be repre- 
sented as

$$
\omega(\mathbf{x})=\omega^{0}(\mathbf{x})+\oint \omega^{6}\left(\mathbf{x} ; \zeta^{6}\right) \lambda\left(\zeta^{6}\right) d S\left(\zeta^{\prime}\right)
$$

where, $\omega^{0}$ and, $\omega^{6}$ are the solutions to

$$
\begin{array}{ll}
(-\Delta+\gamma) \omega^{0}=f & \left.\omega^{0}\right|_{S}=0 \\
(-\Delta+\gamma) \omega^{6}=0 & \omega^{6}\left(\mathbf{x} ; \zeta^{6}\right)=\delta\left(s-\zeta^{6}\right)
\end{array}
$$

for any $\zeta^{6} \in S$ and $\delta$ is the Dirac Delta function over the boundary

The value of the boundary function can then be evaluated by imposing on $\omega$ the integral constraint $\mathrm{Eq} 3.40$ with respect to all harmonic functions on the boundary which are the solution to the following problem

$$
-\nabla^{2} \eta=0 \quad \text { such that } \quad \eta(\mathbf{x} ; \zeta)=\delta(s-\zeta) \quad \text { for any } \zeta \in S
$$

And finally come up with a linear equation of the type $\mathrm{G} \boldsymbol{\lambda}=\boldsymbol{\beta}$

where, the value of the matrix $\mathbf{G}$ is of the form $\int \omega\left(\mathbf{x} ; \zeta^{6}\right) \eta(x ; \zeta) d \Omega$ and can be calculated and stored in the beginning.

The stream function in the above form exists only for flow in two dimensions, so the vorticity-Stream function formulation is rather difficult to implement for a three dimensional flow. Also, the vorticity is now a vector with two tangential components on the boundary. The solenoidal property of the vorticity is no longer implied by its definition but needs to be enforced. It will be shown in the next chapter that the divergence of the vorticity vector in three dimensions can be enforced to be equal to zero by the following two boundary conditions

$$
\begin{gathered}
\left.\boldsymbol{\nabla} \cdot \boldsymbol{\omega}\right|_{S}=0 \\
\left.\boldsymbol{\nabla} \cdot \boldsymbol{\omega}\right|_{t=0}=\boldsymbol{\nabla} \cdot\left(\boldsymbol{\nabla} \times v_{0}\right)
\end{gathered}
$$

But the real problem starts with the boundary conditions for the three dimensional "equivalent" for the stream function for which different schemes involving different vector potentials have been developed, each having its own set of boundary conditions and its own set of elliptic equations to solve. But each method needs a set of boundary conditions for the vector function to ensure its unique solution. Apart from having to solve such complex equations with often debatable boundary conditions, these methods are also not well suited for a variational approach which often turns out to be computationally very expensive. On account of these issues with the three dimensional approach another method has begun to garner interest. This new approach, called the hybrid methods, uses a Vorticity-Velocity 
formulation of the Navier-Stokes equations and seems to be quite well suited to both two dimensional and three dimensional flows. This method will form the basis of this research and shall be covered in the next chapter. 


\section{ThE HYBRID-Method FAMILY AND THE KLE}

\subsection{Hybrid methods}

These methods are based on hybrid formulations in terms of the primitive variable, velocity, and the non-primitive variable, vorticity. They are well suited for two-dimensional as well as three-dimensional flows. Some of the advantages of Vorticity-Velocity $(\boldsymbol{\omega}, \boldsymbol{v})$ formulations compared to the classical formulation with primitive variables or with non-primitive Vorticity-Stream Function methods [6] are:

1. Vorticity is a relevant physical variable which has been extensively studied and its distribution is of immense importance. The velocity is perhaps the most important physical variable which completely defines the kinematical problem at hand, and the fact that they are related by a simple elliptic equation makes this approach all the more advantageous.

2. The velocity field can be supplemented by a unique set of boundary conditions as opposed to a vast number of boundary conditions necessary for a unique solution of the stream vectors or the velocity potentials.

3. The non-inertial terms caused by an accelerating reference frame are introduced into the flow solution through the initial and boundary conditions, without having to do anything extra to evaluate those non-inertial terms.

4. Relative ease of implementing vorticity conditions at infinity as compared to that for pressure.

But hybrid formulation also has some disadvantages too. As already mentioned, the issue with this method is the over-determined kinematic problem and the underdetermined dynamic problem. The unsteady problem in three dimensions has six unknowns compared to 
the four in primitive variable methods. The general formulation can be written as

$$
\begin{gathered}
\frac{\partial \boldsymbol{\omega}}{\partial t}+\boldsymbol{\nabla} \times(\boldsymbol{\omega} \times \mathbf{v})=\nu \nabla^{2} \boldsymbol{\omega} \\
\boldsymbol{\nabla} \cdot \mathbf{v}=0 \\
\boldsymbol{\omega}=\boldsymbol{\nabla} \times \mathbf{v}
\end{gathered}
$$

Many methods involve a Poisson's equation obtained from the curl of Eq4.3 and utilizing Eq4.2 to give

$$
\nabla^{2} \mathbf{v}=-\nabla \times \omega
$$

The major problem here is ensuring the divergence condition as well as the curl of velocity. As mentioned before, the zero divergence of velocity can be ensured throughout the domain by enforcing it on the boundary. But now the solenoidal property for the vorticity also has to be ensured, which can be done in the following way.

It gives the following diffusion by taking the divergence of the transport equation:

$$
\frac{\partial(\boldsymbol{\nabla} \cdot \boldsymbol{\omega})}{\partial t}=\nu \boldsymbol{\nabla}^{2}(\boldsymbol{\nabla} \cdot \boldsymbol{\omega})
$$

Imposing the boundary condition $\left.\boldsymbol{\nabla} \cdot \boldsymbol{\omega}\right|_{S}=0$ on the divergence of vorticity, along with the obvious initial condition $\left.\boldsymbol{\nabla} \cdot \boldsymbol{\omega}\right|_{t=0}=\boldsymbol{\nabla} \cdot\left(\boldsymbol{\nabla} \times \mathbf{v}_{0}\right)$, should give a unique solution to the diffusion equation for $\boldsymbol{\nabla} \cdot \boldsymbol{\omega}$, i.e. $\boldsymbol{\nabla} \cdot \boldsymbol{\omega}=0$ therefore, the solenoidal property is confirmed.

Gatski [71] has classified the solution strategies into method A and method B. Method A "utilizes" the continuity equation $\mathrm{Eq} 4.2$ and the curl Eq 4.3 as the kinematic equations to solve and Eq4.1 as the dynamic transport equation. Method B comprises of solving Eq4.1 and $\mathrm{Eq} 4.4$.

Fasel [14] was among the first to publish numerical results of this method. He used the normal component of the Poisson equation for velocity and the tangential derivative of the continuity equation along with the vorticity transport equation. The boundary condition, in addition to the usual velocity condition, was simply the above-mentioned Poisson's equation on the boundary. Like this, many formulations based on a derived vorticity condition were formulated. Since none of these conditions are genuine constraints, many authors have solved the governing equations without using any vorticity boundary conditions whatsoever. This has led to the use of an integral constraint on the vorticity rather than a local boundary condition.

Since most of the methods do not ensure a solenoidal vorticity field by virtue of the boundary conditions [72], many authors have resorted to using a projection method to ensure the 
solenoidal property of vorticity. Of course, as shown by Wu, et al. [73], the non-solenoidal vorticity can be used to solve for a solenoidal velocity field. However, to find a vorticity field that is solenoidal, the vorticity is decomposed by the Helmholtz theorem to get the Poisson equation,

$$
\nabla^{2} \phi=\nabla \cdot \omega^{0}
$$

where, $\nabla \phi$ is the solenoidal part of the computed vorticity. Once $\nabla \phi$ is solved for from the above equation, the non-solenoidal vorticity can be projected onto the solenoidal field using the relation

$$
\omega=\omega^{0}-\nabla \phi
$$

The next section deals with some of the formulations of the vorticity-velocity methods as presented in [66]

\subsubsection{Hybrid methods in three dimensions}

Using the boundary and the initial conditions mentioned above for vorticity along with the definition $\boldsymbol{\nabla} \times \mathbf{v}=\boldsymbol{\omega}$, the 3-D Vorticity-Velocity formulation can be represented as

$$
\begin{array}{rrr}
\frac{\partial(\boldsymbol{\nabla} \cdot \boldsymbol{\omega})}{\partial t}-\nu \boldsymbol{\nabla}^{2}(\boldsymbol{\nabla} \cdot \boldsymbol{\omega})=0 & \left.\boldsymbol{\omega}\right|_{t=0}=\boldsymbol{\nabla} \times \mathbf{v}_{0} \\
\boldsymbol{\nabla}^{2} \mathbf{v}=-\boldsymbol{\nabla} \times \boldsymbol{\omega} & \mathbf{v}_{S}=\boldsymbol{b} & \boldsymbol{\nabla} \cdot \mathbf{v}_{S}=0 \\
\left.\boldsymbol{n} \cdot \boldsymbol{\omega}\right|_{S}=\left.\boldsymbol{n} \cdot \boldsymbol{\nabla}\right|_{S} \times \boldsymbol{b} & \left.\boldsymbol{\nabla} \cdot \boldsymbol{\omega}\right|_{S}=0
\end{array}
$$

Given the compatibilty conditions $\oint \boldsymbol{n} \cdot \boldsymbol{b} d S=0, \boldsymbol{\nabla} \cdot \mathbf{v}_{0}=0, \boldsymbol{n} \cdot \boldsymbol{b}_{t=0}=\left.\boldsymbol{n} \cdot \mathbf{v}_{0}\right|_{S}$

The above formulation can be solved numerically by a semi-implicit discretization in time and a spectral method with spatial discretization. The lack of boundary values for vorticity can be taken care of by the influence matrix technique as introduced by Daube [74]. This will be briefly introduced in the next section for two dimensional flows along with a temporal discretization scheme to linearize the formulation before resolving it in space.

An uncoupled formulation using an integral constraint can be given as follows,

$$
\begin{array}{rr}
\frac{\partial(\boldsymbol{\nabla} \cdot \boldsymbol{\omega})}{\partial t}-\nu \boldsymbol{\nabla}^{2}(\boldsymbol{\nabla} \cdot \boldsymbol{\omega})=0 & \left.\boldsymbol{\omega}\right|_{t=0}=\boldsymbol{\nabla} \times \mathbf{v}_{0} \\
\int \boldsymbol{\nabla} \times \boldsymbol{\omega} \cdot \boldsymbol{\eta} d \Omega=\oint(\boldsymbol{n} \times \boldsymbol{b} \cdot \boldsymbol{\nabla} \times \boldsymbol{\eta}+\boldsymbol{n} \cdot \boldsymbol{b} \boldsymbol{\nabla} \cdot \boldsymbol{\eta}) d S \\
\left.\boldsymbol{n} \cdot \boldsymbol{\omega}\right|_{S}=\left.\boldsymbol{n} \cdot \boldsymbol{\nabla}\right|_{S} \times \boldsymbol{b} & \left.\boldsymbol{\nabla} \cdot \boldsymbol{\omega}\right|_{S}=0 \\
\boldsymbol{\nabla}^{2} \mathbf{v}=-\boldsymbol{\nabla} \times \boldsymbol{\omega} & \mathbf{v}_{S}=\boldsymbol{b}
\end{array}
$$

Where, $\eta$ is a harmonic vector field defined by the following problem,

$$
-\nabla^{2} \eta=0, \quad \boldsymbol{n} \times\left.\boldsymbol{\eta}\right|_{S}=0
$$

The problem here is the implementation of the the integral constraint in a variational form. The culprits are the boundary conditions $\left.\boldsymbol{n} \cdot \boldsymbol{\omega}\right|_{S}=\boldsymbol{n} \cdot \boldsymbol{\nabla}_{S} \times \boldsymbol{b}$ and $\left.\boldsymbol{\nabla} \cdot \boldsymbol{\omega}\right|_{S}=0$ which cannot 
be used together in a variational formulation.

\subsubsection{Equations in two dimensions}

As shown in the 2-D Vorticity-Stream Function formulation, the vorticity is now a scalar variable given by $\omega=\boldsymbol{\nabla} \times \mathbf{v} \cdot \boldsymbol{k}$ giving the following set of governing equations

$$
\begin{aligned}
& \left(\frac{\partial \omega}{\partial t}-\nu \nabla^{2} \omega\right) \boldsymbol{k}+\nabla \times(\omega \boldsymbol{k} \times \mathbf{v})=0, \quad \omega_{t=0}=\nabla \times \mathbf{v}_{0} \cdot \boldsymbol{k} \\
& \int \omega d \Omega=\oint \boldsymbol{\tau} \cdot \boldsymbol{b} d S \\
& -\nabla^{2} \mathbf{v}=\boldsymbol{\nabla} \omega \times\left.\boldsymbol{k} \quad \boldsymbol{\nabla} \cdot \mathbf{v}\right|_{S}=\left.0 \quad \mathbf{v}\right|_{S}=\boldsymbol{b}
\end{aligned}
$$

Provided the compatibility conditions $\oint \boldsymbol{n} \cdot \boldsymbol{b} d S=0, \boldsymbol{\nabla} \cdot \mathbf{v}_{0}=0, \boldsymbol{n} \cdot \boldsymbol{b}_{t=0}=\left.\boldsymbol{n} \cdot \mathbf{v}_{0}\right|_{S}$ are met. Here $\tau$ is a unit vector tangential to the boundary.

As before, a semi-implicit dicretization can be performed by first discretizing the advection term explicitly, followed by the diffusion terms solved by an implicit scheme such as the Crank-Nicolson scheme.

This gives the following linearized time dicretized formulation,

$$
\begin{array}{rr}
\left(-\nabla^{2}+\gamma\right) \omega^{n+1}=f, & \text { in } \Omega \\
-\nabla^{2} \mathbf{v}^{n+1}=\boldsymbol{\nabla} \omega \times \boldsymbol{k}^{n+1} & \left.\mathbf{v}\right|_{S}=\boldsymbol{b} \\
\boldsymbol{\nabla} \times \mathbf{v}^{n+1}=\omega \boldsymbol{k}^{n+1} \\
\oint_{S} \boldsymbol{b}^{n+1} \cdot \boldsymbol{n} d S=0
\end{array}
$$

Just as for the primitive variable formulation, an influence matrix technique that is devised by Kleiser and Schumann can be used for the Vorticity-Velocity formulation as well.

Solving these methods numerically, as with the three dimensional case, has not been free of problems for both the finite element method and the finite difference method. The integral constraint does offer a better representation of vorticity diffusion and its interaction with solid boundaries, there is an inclination towards using such constraints. Several innovative techniques like the staggered discretization of vorticity by Napolitano and Pascazio [66] have resulted in avoiding certain problems related to a doubly singular influence matrix in the above linear equation. The following sections describe how these problems can be solved by a new method belonging to the hybrid family. 


\subsection{The Kinematic Laplacian Equation method}

The Kinematic Laplacian Equation(KLE) method was first introduced by Ponta [36]. It is a Vorticity-Velocity based method which decouples the evolution of vorticity from the spatial solution of a velocity field. Vorticity is advanced in time by integrating a vorticity transport equation for which an initial velocity field is obtained from the solution of the weak form of the KLE method. The KLE in turn is solved using the vorticity field obtained by integrating the vorticity in time from the previous time step. The no-slip, no-normal flow boundary conditions for velocity required for solving the KLE are solved over a sequence of two steps. This basically involves two integral projection in each time step ensuring compatibility of the two fields at each step. Greater detail is presented in the following sections which are taken from a paper on KLE by F.L. Ponta [75].

\subsubsection{The Constant-Curl Laplacian Equation}

As stated in Ponta and Jacovkis [76], the idea behind using a Laplacian was to come up with a simple linear PDE along the lines of a potential flow equation, however, which could account for rotational effects as seen in turbines as well. This lead to a Kinematic equation for solving time dependant flows over slender bodies with no flow separation under the assumption of incompressible flow and a constant curl.The following vector relation can be used to get the Laplacian of the velocity field

$$
\nabla^{2} \mathbf{v}=\nabla \cdot \nabla \mathbf{v}=\nabla(\nabla \cdot \mathbf{v})-\nabla \times(\nabla \times \mathbf{v}) .
$$

The first and second terms can be ignored on account of the incompressibility and constant curl condition. Thus, the Laplacian $\nabla^{2} \mathbf{v}=0$ can be solved numerically under the incompressibility condition and the constant curl constraint. i.e. $\boldsymbol{\nabla} \cdot \mathbf{v}=0$ and $\boldsymbol{\nabla} \times \mathbf{v}=c$, where $\mathrm{c}$ is a constant.

This earlier version of KLE called the constant curl Laplacian equation (CCLE) [76], had a narrow field of application owing to the constraint of no flow separation. Nevertheless, CCLE was quite successfully used in the study of wind turbine blades [75].

\subsubsection{A generalized Laplacian $(\omega, v)$ method: The KLE}

As mentioned previously, the KLE can be solved to get the spatial distribution of vorticity and velocity. It is a more general PDE expression than the CCLE, not limited to 
non-separated flows. Consider a vorticity velocity formulation for a 3-D Navier-Stokes equation for incompressible viscous flow. Provide a domain with a solid boundary $S$ and a far field external boundary $\Omega$. Therefore in a non-inertial reference frame,

$$
\frac{\partial \boldsymbol{\omega}}{\partial t}=-\mathbf{v} \cdot \boldsymbol{\nabla} \boldsymbol{\omega}+\nu \nabla^{2} \boldsymbol{\omega}+\boldsymbol{\omega} \cdot \boldsymbol{\nabla} \mathbf{v}
$$

If the velocity field is known at a particular time step then the above equation can be written as

$$
\frac{\partial \boldsymbol{\omega}}{\partial t}=-\mathbf{v} \cdot \boldsymbol{\nabla}(\boldsymbol{\nabla} \times \mathbf{v})+\nu \nabla^{2}(\boldsymbol{\nabla} \times \mathbf{v})+(\boldsymbol{\nabla} \times \mathbf{v}) \cdot \boldsymbol{\nabla} \mathbf{v}
$$

This can be integrated in time to solve for $\omega$ at each node using an ODE solver using the vorticity and velocity field evaluated from the previous time step. However, the vorticity field calculated is not compatible with the instantaneous boundary conditions, and the following Laplacian equation is applied to solve for the correct vorticity and velocity fields in the spatial domain:

$$
\begin{gathered}
\nabla^{2} \mathbf{v}=\nabla \mathcal{D}-\nabla \times \omega \\
\nabla \cdot \mathbf{v}=\mathcal{D} \\
\nabla \times \mathbf{v}=\omega
\end{gathered}
$$

As explained in Ponta [36], the KLE is basically a solution of the weak form of Eq 4.17 under the simultaneous imposition of the expansion rate and the curl of the velocity i.e. the vorticity field. Eq4.18 and Eq4.19 give these constraints.

Sections 2.4 to 2.7 of Batchelor [40] gives a good explanation of the physical significance of the two constraints. Most hybrid methods simultaneously solve Eq4.17 and Eq4.16 under the $\boldsymbol{\nabla} \cdot \mathbf{v}=0$ constraint i.e. incompressibility. The KLE, however, as mentioned earlier solves Eq 4.17 independent of the vorticity transport equation. Therefore the vorticity distribution given by $\mathrm{Eq} 4.19$ can be used as a second constraint along with the rate of expansion given by Eq4.18 to solve for the velocity field in space. For a brief validation, consider the decomposition of the velocity field into three orthogonal components: the irrotational component $\mathbf{v}_{\mathcal{D}}$ with zero divergence, the solenoidal component $\mathbf{v}_{\omega}$ with no vorticity and the harmonic component $\mathbf{v}_{h}$. Given the no-normal flow at the boundary along with the vorticity distribution, the above mentioned decomposition i.e. $\mathbf{v}=\mathbf{v}_{\mathcal{D}}+\mathbf{v}_{\omega}+\mathbf{v}_{h}$ has a unique solution [40]. Equation 4.18 and Eq4.19 can be used to solve for $\mathbf{v}_{\mathcal{D}}$ and $\mathbf{v}_{\omega}$ 


$$
\begin{gathered}
\nabla \cdot \mathbf{v}=\nabla \cdot \mathbf{v}_{\mathcal{D}}=\mathcal{D} \\
\nabla \times \mathbf{v}=\nabla \times \mathbf{v}_{\omega}=\omega
\end{gathered}
$$

For $\mathbf{v}_{h}$ substitute the above mentioned decomposition in to $\mathrm{Eq} 4.16$,

$$
\begin{aligned}
\nabla^{2}\left(\mathbf{u}_{h}+\mathbf{v}_{\mathcal{D}}+\mathbf{v}_{\omega}\right) & =\nabla^{2} \mathbf{v}_{h}+\nabla\left(\boldsymbol{\nabla} \cdot \mathbf{v}_{\mathcal{D}}\right)-\nabla \times\left(\boldsymbol{\nabla} \times \mathbf{v}_{\omega}\right) \\
& =\nabla \mathcal{D}-\nabla \times \omega,
\end{aligned}
$$

Substituting Eq4.20 and $\mathrm{Eq} 4.21$ in Eq4.22 gives,

$$
\nabla^{2} \mathbf{v}_{h}=0
$$

This Laplacian equation gives the solution for $\mathbf{v}_{h}$. Therefore the KLE ensures a complete and unique solution of the velocity field.

A solution method based on two consecutive solutions of the KLE is used: the first under free-slip and the second under no-slip boundary conditions on the solid surface. This method yields to imposition of the no-normal flow and no-slip velocity boundary conditions on $S$, together with the correspondingly compatible boundary conditions on the vorticity. The algorithmic sequence explained below [36,75] is iteratively performed at each time step within an iterative time integration performed by an adaptive variable-stepsize ODE solver for incompressible flow.

1. The vorticity is advanced in time by integrating Eq4.16 in time at each node in space to get an initial vorticity field $\tilde{\boldsymbol{\omega}}^{n}$ field. Since velocity from the $(n-1)^{\text {st }}$ time step is used to get vorticity for step $n$, this field is not compatible with the velocity boundary conditions.

2. Enforce homogeneous conditions $\tilde{\boldsymbol{\omega}}^{n}$ at the boundary surface to get $\tilde{\boldsymbol{\omega}}_{0}^{n}$. This is done by imposing a zero boundary value for vorticity at each node on the boundary.

3. Applying the no normal flow velocity boundary conditions and setting $\frac{\partial v_{x}}{\partial \boldsymbol{n}}=0$ on the solid boundary, Eq 4.17 i.e. the KLE is solved for $\overrightarrow{\boldsymbol{v}}^{n}$ under the 2 constraints given by $\mathrm{Eq} 4.18$ and $\mathrm{Eq} 4.19$ using $\tilde{\boldsymbol{\omega}}_{0}^{n}$ as the vorticity field. Here $v_{x}$ is the tangential component of $\boldsymbol{v}$.

4. Using this $\overrightarrow{\boldsymbol{v}}^{n}$ the vorticity field is again calculated as $\boldsymbol{\omega}^{n}=\nabla \times \overrightarrow{\boldsymbol{v}}^{n}$, only this time both boundary conditions i.e. the no slip condition $\boldsymbol{v} \cdot \boldsymbol{\tau}=0$ and the no-normal flow $\boldsymbol{v} \cdot \boldsymbol{n}=0$ are applied on $S$. This $\boldsymbol{\omega}^{n}$ can be seen as a vorticity field produced as an effect of the slip induced in the previous step, somewhat like the vorticity creation methods [77-79]. 
5. Using the above calculated vorticity field $\boldsymbol{\omega}^{n}$ a fixed velocity field $\boldsymbol{v}^{n}$ is computed as a solution to the KLE using both constraints and the two boundary conditions i.e. the no slip condition and the no normal flow.

For the velocity boundary condition on the far field external boundary $S_{\infty}$, the corresponding Dirichlet conditions are applied.

The above algorithm clearly shows the vorticity in time and velocity in space approach of KLE. The momentum equation is solved in step 1 itself. Steps 2-5 consist of solving the KLE for each time step to get the spatial distribution of velocity, compatible with both the vorticity distribution as well as the velocity boundary conditions. Setting vorticity equal to zero at boundary is consistent with the free slip boundary condition for velocity in step 2 and finally as in vorticity creation methods, the no slip condition of step 4 gives the final vorticity field responding to the slip induced in step 3. It can be seen that the two solutions from KLE, each of which is based on a different set of boundary conditions taking care of the vorticity boundary conditions also. These two integral projections on the velocity field ensure a vorticity compatible with the boundary conditions of velocity in each time step. This decoupling between the vorticity evolution and the solution to get velocity distribution along with the compatible vorticity distribution, makes it possible to solve problems with different constitutive relations using this method since the physics involved in any such relation is independent of the spatial solution of KLE. It also becomes much simpler to implement the variational formulation since the PDE system now does not depend either on time or the constitutive relations, but is simply a set of kinematic equations. Since this method is integral and not limited to just the boundary data for calculating boundary vorticity, it does seem to have a somewhat better physical interpretation then the other vorticity generation methods [66].

\subsubsection{Variational formulation of the KLE method}

A variational form of Eq 4.17 can be written using the Galerkin method [75] as follows,

$$
\int_{\Omega}(\boldsymbol{\nabla} \cdot \boldsymbol{\nabla} \mathbf{v}) \cdot \boldsymbol{\delta} \boldsymbol{v} \mathrm{d} \Omega=-\int_{\Omega}(\boldsymbol{\nabla} \times \boldsymbol{\omega}) \cdot \boldsymbol{\delta} \boldsymbol{v} \mathrm{d} \Omega,
$$

where $\delta \boldsymbol{v}$ is a virtual, arbitrary velocity field on $\Omega$ that is set to zero where ever Dirichlet conditions are applied. The next step would be to integrate the left hand side of Eq4.24 by parts and using the divergence theorem to get, $\delta \boldsymbol{v}$ vanishes on $S_{\infty}$,

$$
\int_{\Omega} \nabla \mathbf{v}: \nabla \delta \boldsymbol{v} \mathrm{d} \Omega-\int_{S} \boldsymbol{n} \cdot \boldsymbol{\nabla} \mathbf{v} \cdot \boldsymbol{\delta} \boldsymbol{v} \mathrm{d} S=\int_{\Omega}(\boldsymbol{\nabla} \times \boldsymbol{\omega}) \cdot \boldsymbol{\delta} \boldsymbol{v} \mathrm{d} \Omega
$$


The no slip (as well as the free slip) and no normal flow boundary conditions ensure that $\boldsymbol{\delta} \boldsymbol{v}=0$, thereby reducing $\mathrm{Eq} 4.25$ to:

$$
\int_{\Omega} \nabla \mathbf{v}: \nabla \delta \boldsymbol{v} \mathrm{d} \Omega=\int_{\Omega}(\nabla \times \boldsymbol{\omega}) \cdot \boldsymbol{\delta} \boldsymbol{v} \mathrm{d} \Omega .
$$

The Laplacian operator also has an equivalent minimization formulation which gives for the variational form of KLE the following functional,

$$
\Pi=\int_{\Omega} \frac{1}{2} \nabla \mathbf{v}: \nabla \mathbf{v} \mathrm{d} \Omega-\int_{\Omega}(\boldsymbol{\nabla} \times \boldsymbol{\omega}) \cdot \mathbf{v} \mathrm{d} \Omega .
$$

To impose the constraints in $\mathrm{Eq} 4.18$ and Eq4.19, the penalty method was used over other possible schemes. A brief explanation on why it is preferred over other more rigorous alternatives can be found in [6]. The penalty terms according to the two constraints given by $\mathrm{Eq} 4.18$ and $\mathrm{Eq} 4.19$ are added to Eq4.27 giving the modified functional $\tilde{\Pi}$ as,

$$
\tilde{\Pi}=\Pi+\int_{\Omega} \frac{\alpha_{\mathcal{D}}}{2}(\boldsymbol{\nabla} \cdot \mathbf{v})^{2}+\frac{\alpha_{\omega}}{2}(\boldsymbol{\nabla} \times \mathbf{v}-\boldsymbol{\omega}) \cdot(\boldsymbol{\nabla} \times \mathbf{v}-\boldsymbol{\omega}) \mathrm{d} \Omega
$$

Where the penalty constants are given by $\alpha_{\omega}$ and $\alpha_{\mathcal{D}}$, the stationary of $\tilde{\Pi}$ with respect to $\mathbf{v}$ can be written as,

$$
\begin{gathered}
\delta \tilde{\Pi}=\int_{\Omega} \boldsymbol{\nabla} \mathbf{v}: \boldsymbol{\nabla} \boldsymbol{\delta} \boldsymbol{v}-(\boldsymbol{\nabla} \times \boldsymbol{\omega}) \cdot \boldsymbol{\delta} \boldsymbol{v}+\alpha_{\mathcal{D}}(\boldsymbol{\nabla} \cdot \mathbf{v})(\boldsymbol{\nabla} \cdot \boldsymbol{\delta} \boldsymbol{v}) \\
+\alpha_{\omega}(\boldsymbol{\nabla} \times \mathbf{v}-\boldsymbol{\omega}) \cdot(\boldsymbol{\nabla} \times \boldsymbol{\delta} \boldsymbol{v}) \mathrm{d} \Omega=0 .
\end{gathered}
$$

Reordering the above equation gives,

$$
\begin{aligned}
& \int_{\Omega} \boldsymbol{\nabla} \mathbf{v}: \boldsymbol{\nabla} \boldsymbol{\delta} \boldsymbol{v}+\alpha_{\mathcal{D}}(\boldsymbol{\nabla} \cdot \mathbf{v})(\boldsymbol{\nabla} \cdot \boldsymbol{\delta} \boldsymbol{v})+\alpha_{\omega}(\boldsymbol{\nabla} \times \mathbf{v}) \cdot(\boldsymbol{\nabla} \times \boldsymbol{\delta} \boldsymbol{v}) \mathrm{d} \Omega= \\
& \int_{\Omega}(\boldsymbol{\nabla} \times \boldsymbol{\omega}) \cdot \boldsymbol{\delta} \boldsymbol{v}+\alpha_{\omega} \boldsymbol{\omega} \cdot(\boldsymbol{\nabla} \times \boldsymbol{\delta} \boldsymbol{v}) \mathrm{d} \Omega
\end{aligned}
$$

Equation 4.30 gives the variational formulation for KLE for incompressible flow, with $\mathrm{Eq} 4.18$ and $\mathrm{Eq} 4.19$ as the constraints. As mentioned before, this variational form can be solved by a spatial discretization using finite elements or spectral methods.

Even though in previous paragraphs the KLE was referred to as a "vorticity-intime/velocity-in-space split approach," this is more a general description of its timespace/vorticity-velocity uncoupled nature than a strict definition of its algorithmic structure. Strictly speaking, time-marching splitting or fractional-step methods replace simultaneous processes by sequential steps as a means to increase efficiency [80]. Split may be by dimensions (e.g. a three-dimensional process split into three one-dimensional substeps), or by physics (e.g. advection on one fractional step, pressure adjustment on another, and diffusion on a third). For the hydrodynamic equations, the advantage of splitting-by-process is that the nonlinear advection process can be treated by a different algorithm than pressure adjustment, which in turn can be different from diffusion. The latter two each involve a linear solution. The advective step is usually advanced explicitly and the adjustment of fields is integrated implicitly. A typical example of this technique is the very successful AB3CN (third-order Adams-Bashforth/Crank-Nicholson) three-step scheme (see Sherwin and Karniadakis [81],Thompson et al. [82], among others). Besides its advantages, splitting 
also has some drawbacks, mostly related with consistency and the treatment of boundary conditions (see Boyd [80], Sec. 13.1-13.4). The choice of appropriate boundary conditions is quite important in minimizing the splitting error, as shown by Karniadakis et al. [83], where high-order pressure boundary conditions are found to be the key to achieve the accuracy in time of the splitting scheme.

Contrarily, there is no splitting whatsoever in the KLE method. All terms in the physical problem are solved simultaneously during time integration of the vorticity field, and all the spatial components of the velocity are solved together by the KLE. Since the KLE is an entirely Kinematic equation with the entire physics concerned with any of the nonlinearities and complex constitutive relations limited to the time integration schemes, it favors modeling complex flow problems like non-Newtonian flows or turbulent flows, etc. Since it is also a universal vector equation, basically any field represented by this relation can be solved for as long as the divergence and curl of that field has a solvable transport equation. Also, since the vector relation is independent of the time iteration process, other processes such as heat transfer or chemical processes can be coupled with the KLE method by simply joining the required relation to the existing ODE system. So basically just the source term to the KLE is changed to solve different physical problems.

\subsection{Numerical implementation of the KLE method}

This section deals with the numerical implementation of the KLE by applying a spectral element method to discretize Eq 4.30 in space along with a predictor-corrector time integration scheme. The spatial discretization scheme used here is a two-dimensional isoparametric spectral element with a high order Lagrangian polynomial to interpolate solutions within the element. An isoparametric element was chosen because of the complex differential equations involved and the complexity of the intended surfaces to be modeled. The main advantage of this element is that the integration has to be performed over the "parent" element which represents a normalized domain in terms of a local co-ordinate system varying between +1 and -1 . This makes it easier to implement any numerical technique. An isoparametric element uses the same Lagarange polynomial (shape functions) to interpolate the unknown variable within the elements as the ones used to map the global to local coordinates. Figure 4.1 shows an example of a nine-node isoparametric quadrilateral element on its natural system of coordinates, i.e. $(\mathrm{r}, \mathrm{s})$ based on the biquadratic interpolation

functions. A quadrilateral element is comparable to linear elememts because it has high convergence rate and the ability to reduce the skin error on curvilinear boundaries. Nevertheless, the implementation of the KLE method may use other discretization techniques. For Ponta's experimentation, the spectral element method is used for the KLE which will be discussed in the following section. 


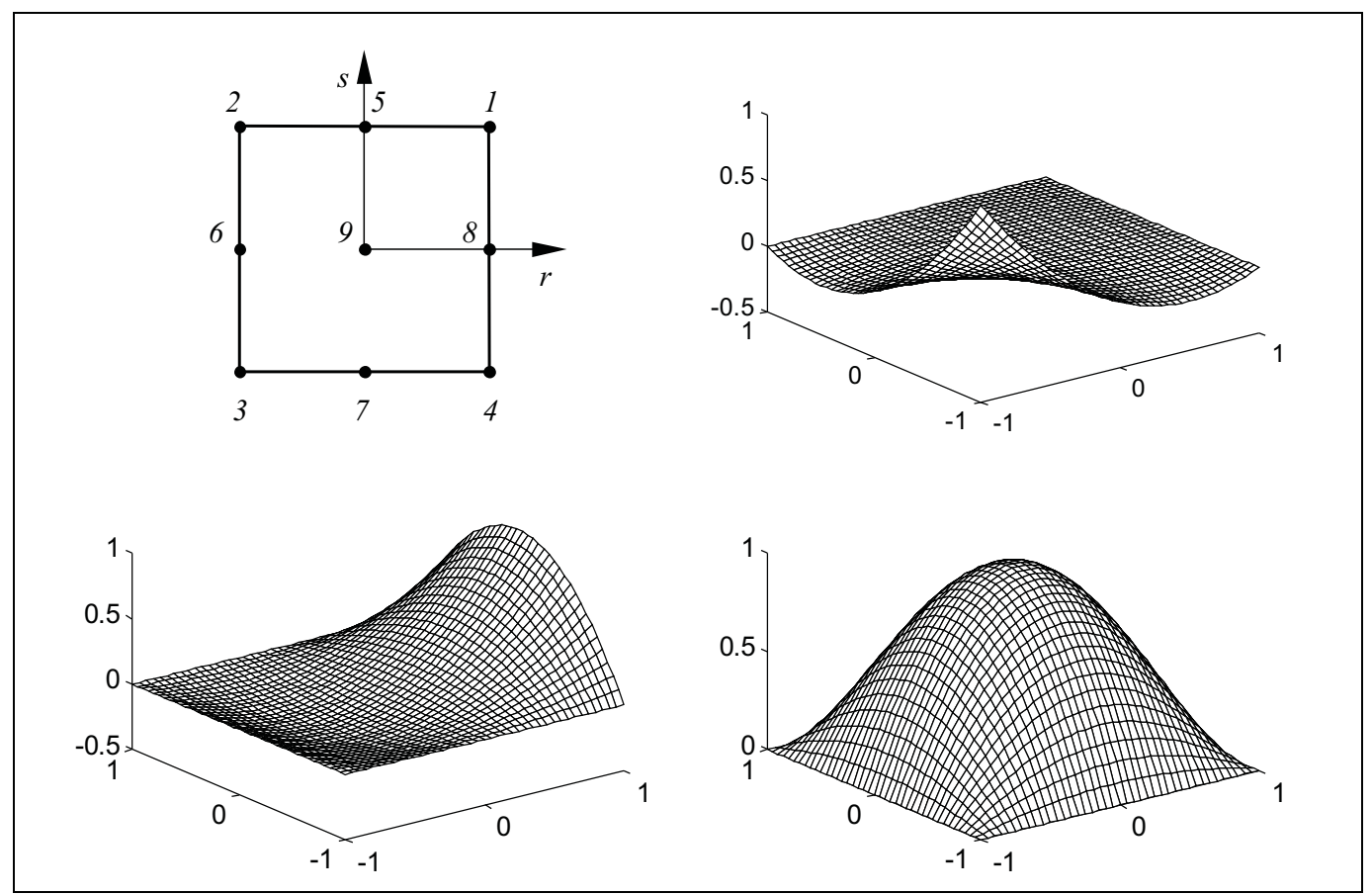

Figure 4.1. A two-dimensional nine node isoparametric element in its natural coordinate system along with a graphical representation of three of its nine interpolation functions i.e nodes 3,8 and 9, [Figure created by author from data in 6]. 


\subsubsection{The Spectral-element method for KLE}

The general trend in finite element methods had been to use Lagrange polynomials of a particular order as shape functions. The total number of elements was increased in order to improve the accuracy. This is called an $h$-type finite element method. For sufficiently smooth problems these methods converge at an algebraic rate with the error being proportional to $\frac{1}{N^{p+1}}$ where $N$ is the number of degrees of freedom and $p$ is the order of the Lagrange polynomial [84]. Another approach would be to follow the $h$-type discretization with an increase in the order of the interpolating polynomial within each element to improve accuracy. Again, for sufficiently smooth solutions this would give an exponential convergence rate [84]. These are called the $p$-type methods. The spectral method is a particular implementation of the $p$-version of an $h p$ finite element method.

The spectral-element method was introduced some twenty years back [85,86]. Its main purpose was to tackle complicated domains which the spectral methods were not able to handle. As shown in Henderson and Karniadakis [87], this $h-p$ type of method was capable of local refinements, and where thus good dealing with the complex geometries and meanwhile preserved the high convergence rates seen in spectral methods. Owing to the $h-p$ discretization, a high accuracy can be achieved for fewer nodes, making it a highly memory-minimizing method [80].

Mostly the Legendre or Chebyshev polynomials are used by the spectral element methods in order to come up with suitable basis functions. The same points are used for the interpolation functions as for the numerical integration within the elements. These collocation points are called the Gauss-Legendre-Lobatto (GLL) quadrature points. This leads to diagonal mass matrices making the system more efficient.

As mentioned at the beginning of this chapter, in this particular analysis an isoparametric element is used with the Lagrangian polynomials as interpolating functions for the solution. The variational formulation for the KLE using the Galerkin method, shown in the previous chapter, is used to solve for the velocity field at the nodal points. The nodes are at the GLL points. For higher order elements using the GLL points in place of the regular equispaced points is more economical [88]. Giraldo [89], through experiments has shown that for higher order interpolating polynomials (in excess of 4) the solution results for Gauss Legendre and GLL quadrature are comparable.

As shown in Ponta and Jacovkis [75], the finite-element discretization of the velocity field 
and its gradient can be represented as,

$$
\mathbf{v}=\left[\begin{array}{l}
v_{x} \\
v_{y}
\end{array}\right]=\mathbf{H} \cdot \mathbf{U}^{e}, \quad \quad \nabla \mathbf{v}=\left[\begin{array}{c}
\frac{\partial v_{x}}{\partial x} \\
\frac{\partial v_{x}}{\partial y} \\
\frac{\partial v_{y}}{\partial x} \\
\frac{\partial v_{y}}{\partial y}
\end{array}\right]=\mathbf{B} \cdot \mathbf{U}^{e}
$$

where $\mathbf{H}$ is the interpolation-function, $\mathbf{B}$ it's derivative and $\mathbf{U}^{e}$ is the array of discretized velocity at nodes of each element, 


$$
\begin{aligned}
& \mathbf{U}^{e}=\left[\begin{array}{c}
v_{x}^{1} \\
v_{y}^{1} \\
v_{x}^{2} \\
\vdots \\
v^{N G L^{2}}
\end{array}\right], \quad \mathbf{H}=\left[\begin{array}{cccccc}
h^{1} & 0 & h^{2} & \cdots & h^{N G L^{2}} & 0 \\
0 & h^{1} & 0 & \cdots & 0 & h^{N G L^{2}}
\end{array}\right] \text {, } \\
& \mathbf{B}=\left[\begin{array}{cccccc}
\frac{\partial h^{1}}{\partial x} & 0 & \frac{\partial h^{2}}{\partial x} & \cdots & \frac{\partial h^{N G L^{2}}}{\partial x} & 0 \\
\frac{\partial h^{1}}{\partial y} & 0 & \frac{\partial h^{2}}{\partial y} & \cdots & \frac{\partial h^{N G L^{2}}}{\partial y} & 0 \\
0 & \frac{\partial h^{1}}{\partial x} & 0 & \cdots & 0 & \frac{\partial h^{N G L^{2}}}{\partial x} \\
0 & \frac{\partial h^{1}}{\partial y} & 0 & \cdots & 0 & \frac{\partial h^{N G L^{2}}}{\partial y}
\end{array}\right]
\end{aligned}
$$

where $N G L=p+1$ is the number of nodes of the Gauss-Lobatto interpolation.

The elements of Eq4.33 are given by,

$$
\left[\begin{array}{c}
\frac{\partial h^{k}}{\partial x} \\
\frac{\partial h^{k}}{\partial y}
\end{array}\right]=\mathbf{J}^{-1} \cdot\left[\begin{array}{c}
\frac{\partial h^{k}}{\partial r} \\
\frac{\partial h^{k}}{\partial s}
\end{array}\right], \quad k=1, \ldots, N G L^{2},
$$

where $\mathbf{J}$ is the Jacobian operator which relates the natural to the local coordinate derivates,

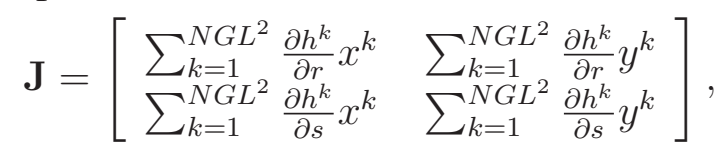


and $\left(x^{k}, y^{k}\right)$ the local coordinates of the nodes. The divergence of the velocity field is given by

$$
\boldsymbol{\nabla} \cdot \mathbf{v}=\mathbf{m} \cdot \mathbf{B} \cdot \mathbf{U}^{e}, \quad \mathbf{m}=\left[\begin{array}{llll}
1 & 0 & 0 & 1
\end{array}\right],
$$

and the curl of the velocity $\omega_{z}$ (the only non-zero component of the curl), is obtained as,

$$
\boldsymbol{\nabla} \times \mathbf{v}=\mathbf{r} \cdot \mathbf{B} \cdot \mathbf{U}^{e}, \quad \mathbf{r}=\left[\begin{array}{llll}
0 & -1 & 1 & 0
\end{array}\right] .
$$

Similarly for vorticity,

$$
\boldsymbol{\omega}=\mathbf{H}_{\omega} \cdot \boldsymbol{\omega}^{e}, \quad \boldsymbol{\nabla} \times \boldsymbol{\omega}=\left[\begin{array}{c}
\frac{\partial \omega}{\partial y} \\
-\frac{\partial \omega}{\partial x}
\end{array}\right]=\mathbf{B}_{\omega} \cdot \boldsymbol{\omega}^{e},
$$

where $\omega^{e}$ gives the vorticity values at nodes of each element calculated by integrating in time the vorticity transport equation, and as shown for velocity $\mathbf{H}_{\omega}$ and $\mathbf{B}_{\omega}$ are the vorticity interpolation-functions and their derivatives respectively,

$$
\begin{gathered}
\boldsymbol{\omega}^{e}=\left[\begin{array}{c}
\omega^{1} \\
\omega^{2} \\
\vdots \\
\omega^{N G L^{2}}
\end{array}\right], \quad \mathbf{H}_{\omega}=\left[\begin{array}{llll}
h^{1} & h^{2} & \ldots & h^{N G L^{2}}
\end{array}\right], \\
\mathbf{B}_{\omega}=\left[\begin{array}{cccc}
\frac{\partial h^{1}}{\partial y} & \frac{\partial h^{2}}{\partial y} & \cdots & \frac{\partial h^{N G L^{2}}}{\partial y} \\
-\frac{\partial h^{1}}{\partial x} & -\frac{\partial h^{2}}{\partial x} & \cdots & -\frac{\partial h^{N G L^{2}}}{\partial x}
\end{array}\right] .
\end{gathered}
$$

For the finite element analysis, first each element can be thought of as a discretized subdomain $\left(\Omega^{e}\right)$. Therefore if Eq4.30 is considered at each $\Omega^{e}$ and the corresponding discretized values of the velocity and vorticity fields are substituted for, the following equation is obtained,

$$
\boldsymbol{\delta} \mathbf{U}^{e^{T}} \cdot \underbrace{\left(\mathbf{K}_{L}^{e}+\mathbf{K}_{\mathcal{D}}^{e}+\mathbf{K}_{\omega}^{e}\right)}_{\mathbf{K}^{e}} \cdot \mathbf{U}^{e}=\boldsymbol{\delta} \mathbf{U}^{e^{T}} \cdot \underbrace{\left(\mathbf{R}_{L}^{e}+\mathbf{R}_{\omega}^{e}\right)}_{\mathbf{R}^{e}} \cdot \boldsymbol{\omega}^{e},
$$

where

$$
\begin{aligned}
& \mathbf{K}_{L}^{e}=\int_{\Omega^{e}} \mathbf{B}^{T} \cdot \mathbf{B} \mathrm{d} \Omega=\int_{-1}^{1} \int_{-1}^{1} \mathbf{B}^{T} \cdot \mathbf{B}|\mathbf{J}| \mathrm{d} r \mathrm{~d} s \\
& \mathbf{K}_{\mathcal{D}}^{e}=\int_{-1}^{1} \int_{-1}^{1} \alpha_{\mathcal{D}} \mathbf{B}^{T} \cdot \mathbf{m}^{T} \cdot \mathbf{m} \cdot \mathbf{B}|\mathbf{J}| \mathrm{d} r \mathrm{~d} s \\
& \mathbf{K}_{\omega}^{e}=\int_{-1}^{1} \int_{-1}^{1} \alpha_{\omega} \mathbf{B}^{T} \cdot \mathbf{r}^{T} \cdot \mathbf{r} \cdot \mathbf{B}|\mathbf{J}| \mathrm{d} r \mathrm{~d} s \\
& \mathbf{R}_{L}^{e}=\int_{-1}^{1} \int_{-1}^{1} \mathbf{H}^{T} \cdot \mathbf{B}_{\omega}|\mathbf{J}| \mathrm{d} r \mathrm{~d} s \\
& \mathbf{R}_{\omega}^{e}=\int_{-1}^{1} \int_{-1}^{1} \alpha_{\omega} \mathbf{B}^{T} \cdot \mathbf{r}^{T} \cdot \mathbf{H}_{\omega}|\mathbf{J}| \mathrm{d} r \mathrm{~d} s
\end{aligned}
$$

$\boldsymbol{\delta} \boldsymbol{U}^{e}$ gives the array of values at nodes of each element for the arbitrary $\boldsymbol{\delta} \boldsymbol{v}$. 

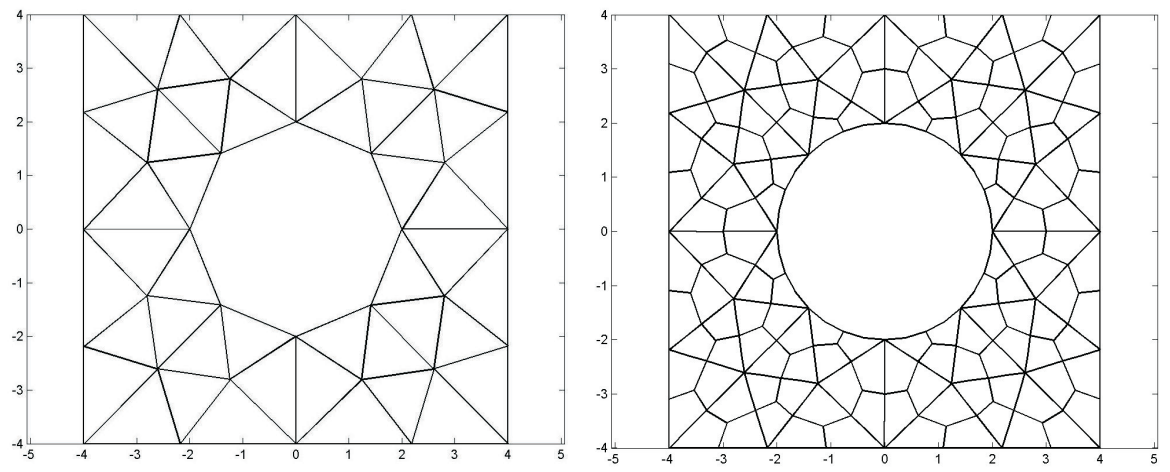

Figure 4.2. A tri-quadrilateral finite element mesh derived from an unstructured triangular mesh, [Figure created by author from data in 6].

The arrays and the matrices of Eq4.41 are assembled for each element to give the following global system,

$$
\mathbf{K} \cdot \mathbf{U}^{e}=\mathbf{R} \cdot \boldsymbol{\omega} .
$$

As mentioned earlier, a quadrilateral element has high convergence rate and reduces the skin error on circular boundaries. At the same time triangular elements make it easier to change mesh density in a more smooth and gradual manner and are also more suitable for unstructured meshing [75,76]. Thus the domain was first dicretized using triangular elements which was subsequently converted to a quadrilateral mesh, by dividing each triangle into three quadrilaterals. This can be seen in Figure 4.2.

An important advantage of this "tri-quadrilateralization" is a process called static condensation of internal nodes, see in Figure 4.3. These nodes lying inside the triangle though used for elemental integration are not used while assembling the final global structure matrices. These are later recovered from the values obtained by the solution of the external nodes.

To establish an equation for this condensation, as shown in [90], the system $\mathbf{K}^{e} \cdot \mathbf{U}^{e}=$ $\mathbf{R}^{e} \cdot \boldsymbol{\omega}^{e}$ are partitioned as,

$$
\left[\begin{array}{ll}
\mathbf{K}_{a a}^{e} & \mathbf{K}_{a b}^{e} \\
\mathbf{K}_{b a}^{e} & \mathbf{K}_{b b}^{e}
\end{array}\right] \cdot\left[\begin{array}{c}
\mathbf{U}_{a}^{e} \\
\mathbf{U}_{b}^{e}
\end{array}\right]=\left[\begin{array}{c}
\mathbf{R}_{a}^{e} \\
\mathbf{R}_{b}^{e}
\end{array}\right] \cdot \boldsymbol{\omega}^{e}
$$

where $a$ is for the degrees of freedom 1-24 of the velocity at nodes 1-12 and $b$ is for the degrees of freedom 25-38 of the velocity at nodes 13-19. The second row of the above equation gives,

$$
\mathbf{U}_{b}^{e}=\underbrace{\left(\mathbf{K}_{b b}^{e}\right)^{-1} \cdot \mathbf{R}_{b}^{e}}_{\mathbf{R}_{b}^{e}} \cdot \boldsymbol{\omega}^{e}-\underbrace{\left(\mathbf{K}_{b b}^{e}\right)^{-1} \cdot \mathbf{K}_{b a}^{e}}_{\mathbf{K}_{b a}^{e}} \cdot \mathbf{U}_{a}^{e},
$$




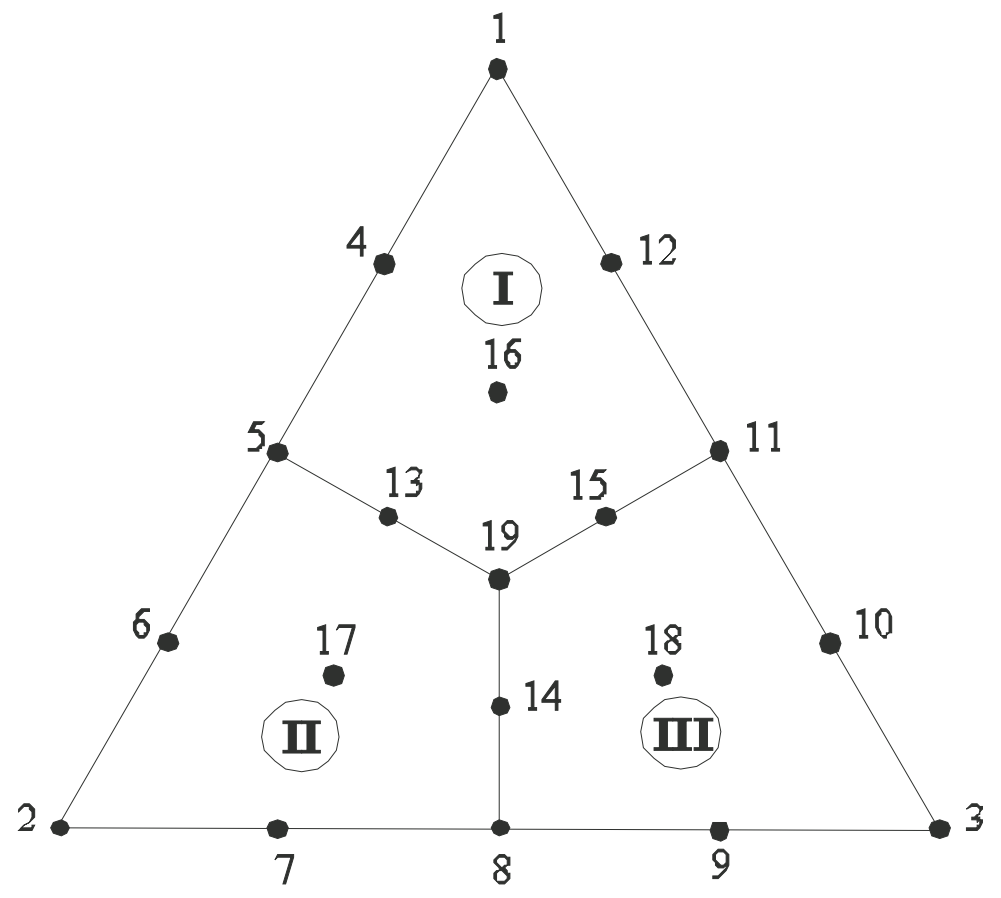

Figure 4.3. The internal topology of a tri-quadrilateral element. Quadrilateral elements (I)-(III) are the nine-node isoparametric elements, [Figure created by author from data in 6]. 1-19 is the in-triangle global numbering of the nodes. 
substituting this into the first row of $\mathrm{Eq} 4.43$ and reordering,

$$
\begin{aligned}
& \underbrace{\left(\mathbf{K}_{a a}^{e}-\mathbf{K}_{a b}^{e} \cdot\left(\mathbf{K}_{b b}^{e}\right)^{-1} \cdot \mathbf{K}_{b a}^{e}\right)}_{\mathbf{K}^{e}} \cdot \mathbf{U}_{a}^{e}= \\
& \underbrace{\left(\mathbf{R}_{a}^{e}-\mathbf{K}_{a b}^{e} \cdot\left(\mathbf{K}_{b b}^{e}\right)^{-1} \cdot \mathbf{R}_{b}^{e}\right)}_{\mathbf{R}^{e}} \cdot \boldsymbol{\omega}^{e},
\end{aligned}
$$

This is the condensed form. Assembling the arrays and matrices of Eq 4.44 and Eq 4.45 gives the following global condensed system,

$$
\begin{gathered}
\mathbf{K} \cdot \mathbf{U}_{a}=\mathbf{R} \cdot \boldsymbol{\omega}, \\
\mathbf{U}_{b}=\mathbf{R}_{b} \cdot \boldsymbol{\omega}-\mathbf{K}_{b a} \cdot \mathbf{U}_{a},
\end{gathered}
$$

The static condensation process reduces almost $40 \%$ in the size of the global system to be solved and also leads to a better condition number of the global structure matrices. This is in accordance with the Schur complement method, where the condensed matrix forms the Schur complement for the in-triangle nodes of the original system. As mentioned earlier none of the structure matrices depend on the physics of the problem, in this case the vorticity or the time, they can be calculated and stored and used over and over again as required. Since KK is positive definite and symmetric, it is factorized by the Cholesky decomposition method and the factor (triangular) so obtained can be used to solve for $\mathbf{U}_{a}$. One problem with spectral element methods is that the loss of the exponential convergence and also the higher accuracy in case of singularities like shock in compressible flow [91]. This is often seen while interpolating non-smooth functions (abrupt changes in boundaries and forces etc.) using high order polynomials.

\section{Evaluating the right-hand side of the vorticity transport equation}

As shown in Ponta and Jacovkis [75], for the two-dimensional implementation of the integration procedure in time, the vorticity transport Eq4.16 can be written in a more convenient way as follows,

$$
\frac{\partial \boldsymbol{\omega}}{\partial t}=\boldsymbol{F}(\boldsymbol{\omega}, t)=\boldsymbol{\nabla} \times(\nu \boldsymbol{\nabla} \cdot \boldsymbol{\nabla} \mathbf{v}-\mathbf{v} \cdot \boldsymbol{\nabla} \mathbf{v}) .
$$

The RHS of Eq4.48 is solved for by carrying out the respective curl, divergence and gradient operations on the discretized counterpart of $u$ as found by the KLE algorithm previously explained. Since, for the spectral-element case, the Gauss-Lobatto points are the same as the nodes, therefore, for those lying on the inter-element boundaries, an average of the values from elements sharing those boundaries can be used.

The weight of each Gaussian point depends on the mesh geometry and is calculated during assembly. So the arrays for the differential operators are assembled at the same time as the Finite element matrices. Those arrays perform the differential operations on any vector 
or tensor field, as a dot product with the corresponding discrete solution of that field. For instance, the discrete form of the curl of the velocity field $\nabla \times \mathbf{v}$ is given by the dot product $C_{u r l} \cdot \mathrm{U}$. Thus, the discrete form of Eq4.48 is written as,

$$
\mathbf{F}(\boldsymbol{\omega}, t)=\mathbf{C}_{u r l} \cdot\left(\nu \mathbf{D}_{i v}-\mathbf{U}_{a d v}\right) \cdot \mathbf{G}_{r a d} \cdot \mathbf{U},
$$

where $\mathbf{G}_{r a d}$ gives the gradient, $\mathbf{D}_{i v}$ the divergence of $\mathbf{G}_{r a d}, \mathbf{C}_{u r l}$ the curl vector and $\mathbf{U}_{a d v}$ is obtained by reordering $\mathrm{U}$ to perfrom the dot product $\mathbf{v} \cdot \nabla \mathbf{v}$ in the advective term.

Since none of them depend on the vorticity field or time, they can be, as with the structure matrices, calculated and stored for further use. For the time integration Adams-BashforthMoulton predictor-corrector (ABM-PECE) solver with multivariable order and adaptive stepsize is used and the results show that it is highly efficient for this application.

\section{The adaptive time step solver for KLE}

As the name suggests, the adaptive time step solver technique receives the response from the calculated variables and then the initial or intermediate values modify themselves to the next refined value. The adaptive stepsize techniques are employed to control the accuracy of the simulations and to enhance their efficiency to provide stable steady state or transient solutions. The adaptive time-step algorithms usually use the values of approximate local truncation error or some of them are even based on some kind of "Thumb Rule". In some of the standard algorithms, user is required to specify the accuracy requirement on local truncation error which is compared with computed values of the same which should be within accepted accuracy or tolerance range [92].

As mentioned in a previous section, a very robust implementation of the Adams-BashforthMoulton predictor-corrector (ABM-PECE) solver with adaptive stepsize control has been used with KLE successfully. The solver ODE113 in MATLAB, which is based on ABM$\mathrm{PECE}$, is used for the numerical experiments on vortex dynamics presented in this thesis.

The MATLAB code ODE113 was derived from the well-known code STEP [93]. For more explanation about code STEP, see the original research paper by L.F. Shampine and M.K. Gordon. ODE113 is the modification to the code STEP and it is a variable step-size, variable order and compiled using ABM-PECE method with local extrapolation. The modified divided difference form of the interpolating polynomials is used in this method. It means when step size is constant, they are just normal backward differences. The acceptance or rejection of the time step is largely dependent upon the local error estimate which is nothing but the difference between the two corrector formula of two consecutive orders. This local error estimate is calculated and compared using the inbuilt checking criteria, before the final evaluation in the $\mathrm{P}_{k} \mathrm{EC}_{k+1} \mathrm{E}$ method. Thus, a rejected time step only requires one functional evaluation. The order from 1 to 12 can be altered in this code [94]. 


\section{NUMERICAL EXPERIMENTATION}

\subsection{Wake dynamics behind bluff bodies at low Re}

Many regular and irregular flow patterns have been experimentally observed in the development and decay of the disturbed flow structures behind circular cylinders. These experiments have studied the separation of bubbles at low $\mathrm{Re} ; R e_{o s c}$, the Re above which oscillations are always found; and the wake frequency. Of principal importance in these studies are the measures used to minimize influencing parameters such as turbulence, surface roughness, wall blockage, i.e. These effects are particularly important at the ends of the cylinder.

\section{Division of laminar flow regimes}

It is desired to determine a range of Re that a given characteristic flow will exist. A well defined boarder of a given region is not possible due to unaccounted disturbances in the flow. The laminar flow region between 0 and 200 is typically divided into three regimes [37]. The first regime is a non-separation or creeping flow regime at $0<R e<4$ to 5 . A creeping flow is cleanly attached to the circumference of the cylinder and a steady and symmetric laminar shear layer does not form a visible wake. The transition to the second regime begins with $R e_{s}, R e_{s}$ is the $\mathrm{Re}$ at which the boundary layers separate. Various sources have estimated $R e_{s}$ to be between 4.3 for a low $D / B$ [95] and up to 9.6 for the case of $D / B=0.12$ [96], B is the channel width. The second regime is defined by steady separation or closed near-wake at 4 to $5<R e<30$ to 48 . These separated free shear layers meet downstream at the confluence point where a reverse velocity along the axis of the closed near-wake is formed. The reverse velocity is normally one order of magnitude lower than the free stream velocity. At $R e_{o s c}$, around 30 to 48, the closed near-wake becomes unstable and a sinusoidal oscillation of the shear layers is formed at the confluence point. This phenomenon is considered as the transition into the 3rd regime, a periodic laminar flow regime. The transition continues up to $R e_{v s}$ around 45 to 65 where the free shear

The material contained in this chapter is original and in preparation for publication 
layers begin to roll up into vortices. When Re is greater than $R e_{v s}$, the near wake forms a periodic eddies street and sheds into downstream. This phenomenon continues to around $R e=200$. In this work the second closed near-wake regime and the transition to the third or periodic laminar regime were studied. Flows around circular cylinders at low $\mathrm{Re}$ have already been thoroughly studied experimentally, primarily by Camichel et al. [97], Camichel and Teissié-Solier [98], Taneda [12 99] and later by Coutanceau and Bouard [96] and Gerrard [100].

\section{Geometry of closed near-wake}

As Re increases beyond $R e_{s}$, the shear layers become separated and then merge downstream to form a symmetric steady and closed near-wake. Zdravkovich [37] defined the Re range for this regime at $5<\mathrm{Re}<40$ and observed a steady separated region in the form of a laminar closed near-wake. Williamson [101] observed the recirculation regions downstream of the body and a pair of symmetrically placed vortices on either side of the wake which grow with Re. Williamson [101] however defined the region as $\operatorname{Re}<49$.

By coating a cylinder with condensed milk, Taneda [12] has been able to visualize the flow in this regime. At $R e=28.4$, Figure 5.1 shows the boundary of the recirculating region located between the separated shear layers. In Figure 5.2, the experiment is repeated at Taneda's [12], defined upper limit for the steady flow, $\mathrm{Re}=41$. Here tiny irregular gathers which appeared on the boundary of the recirculating region can be seen before they die out further downstream. Far Downstream from the gathers, a sinusoidal oscillation can be seen, implying the start of the transition to periodic laminar flow. Camichel et al. [97] photographed a remarkable evolution in the topologies of the near-wake for Re between 20 and 40. This series of photos captures the elongation and destruction of the initial closed near-wake. Then a new near-wake is accomplished by a secondary separation of the free shear layers. This new near-wake is then filled with dye as the separated shear layers converge and merge. Finally when $R e=40$, a steady near-wake is maintained. If the assumptions from Föppl [102] were correct, an increase in Re would have increased the size of the original eddies rather than destructing them as was shown in Camichel's results.

The length of the closed near-wake has been measured by various authors. Coutanceau and Bouard [96] eliminated the effect of wall blockage by extrapolating results to an infinitely wide channel. This length is taken as the distance from the downstream end of the bluff to the end of the bubble. A pressure gradient along the channel, converging or diverging, could have a significant effect on the ratio of $L_{w}$ to D, as proposed by Honji [103]. The empirical relationship is shown as a linear function of Re,

$$
\frac{L_{w}}{D}=0.05 \times R e, \text { for } 4.4<R e<40
$$




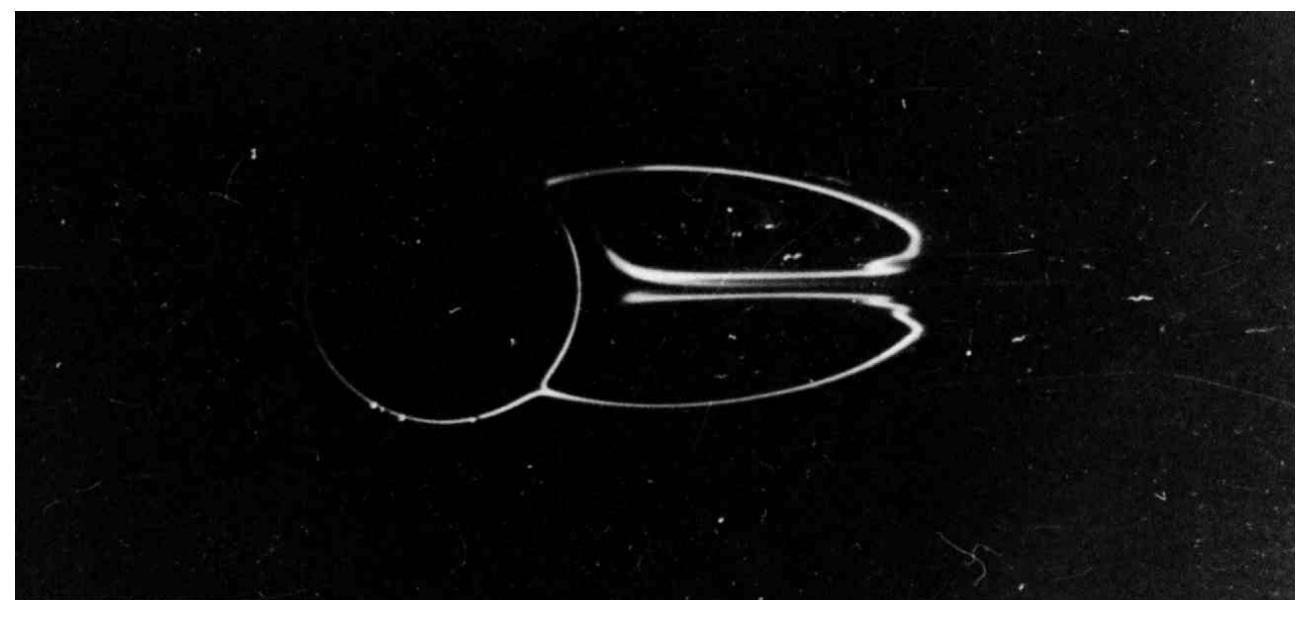

Figure 5.1. Closed near-wake behind circular cylinder at $\mathrm{Re}=28.4$ [taken from 12]

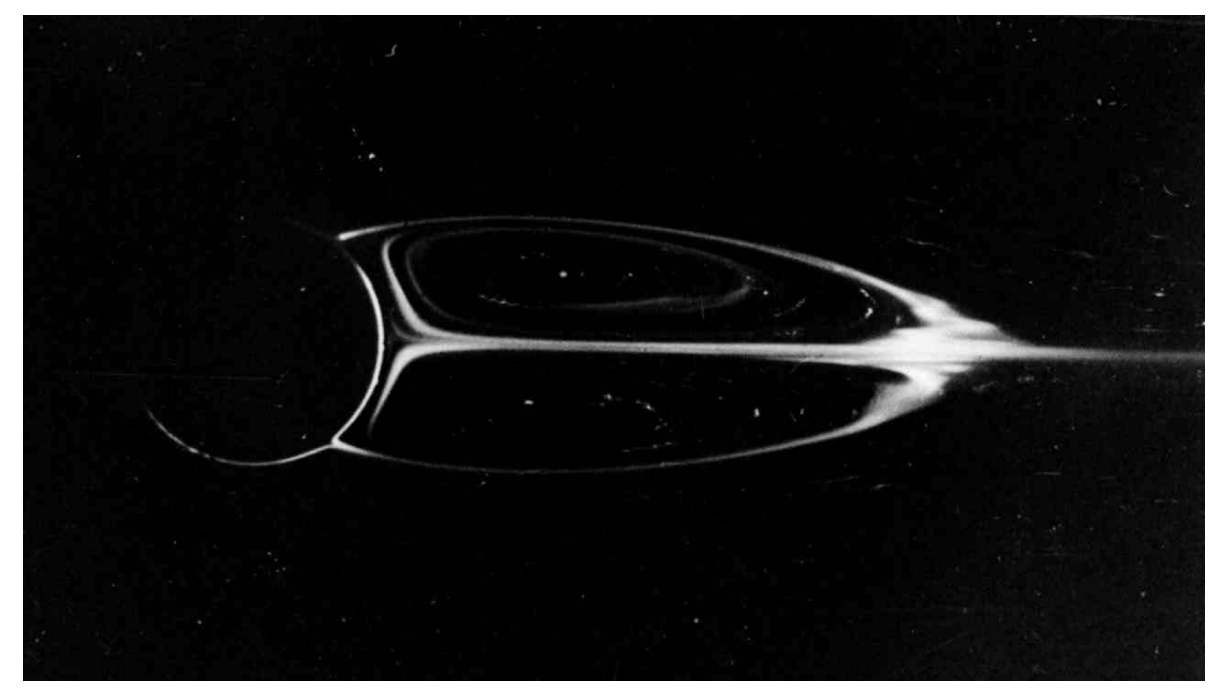

Figure 5.2. Closed near-wake behind circular cylinder at $\mathrm{Re}=41$ [taken from 12 ] 


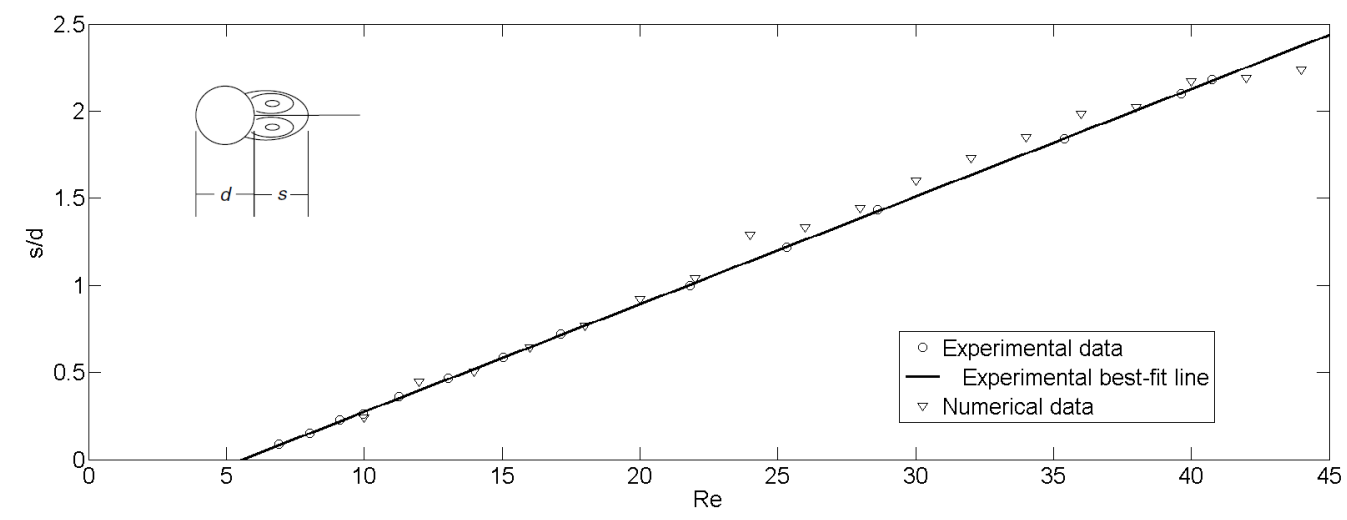

Figure 5.3. Comparison of the wake length computed numerically with Taneda's experimental data.

Where $L_{w}$ is the length of the closed near-wake.

The shape of the closed near-wake boundary is dependent on Re. In an experiment by Thom [104], a cusped short near-wake was formed for $R e<7.4$ and the maximum width was located between the separation points. When $R e=7.4$, the widest point moved downstream but did not pass the centres of recirculating cores. Unlike the rest of the nearwake boundary, the portion of the near-wake behind the maximum width is scalable to the length of the near-wake for the range $20<R e<40$ [96].

The ratio of streamwise distance, eddy center to cylinder, and transverse distance, eddy center to near-wake axis was measured at $D / E$ of $0,0.04$ and 0.12 by Grove et al. [105] and Coutanceau and Bouard [96]. These were then scaled to the diameter of the cylinder. From this it was seen, the streamwise distance increased linearly with Re. A surprising feature of the closed near-wake regime can be seen in the streamlines displaced by the cylinder. Thom [104] and Homann [106] reported that these streamlines do not follow the shape of the closed near-wake boundaries but rather they diverge further away from the cylinder. To quantify this phenomenon, two streamlines that were observed an initial distance apart became displaced and separated downstream. It was also noted that the transverse displacement of streamlines decreases with increasing Re but the location of maximum displacement moves further downstream. The sensitivity of the wake length to the blockage ratio can be seen by observing the separate curves developed by Thom [104] and Homann [106].

Figure 5.3 shows the numerically computed $L_{w}$ from the KLE method with respect to Re. It shows a favourable agreement with the experimental measurements by Taneda [12]. 


\section{Closed near-wake instability}

Many researches have been done on the cases when $R e_{o s c} \geq R e \leq R e_{v s}$. The wake oscillation in the near-wake starts at $R e_{o c s}$ and continues right before the onset of the wake sheds at $R e_{v s}$. Some values of the $R e_{o s c}$, which are widely scattered depending on the experimental set-ups even when the blockage effect are negligible, are reported by Taneda [12], Coutanceau and Bouard [96], Gerrard [100], Kovasznay [107], Roshko [55] and Nishioka and Sato [108]. These $R e_{\text {osc }}$ are 30, 34, and 33 from flow visualization and 40, 40, and 48 from hot-wire measurements. The higher values of $R e_{o s c}$ from the latter may be owing to the sensitivity of a single hot wire to a periodic change in the direction of velocity. Kovasznay [107] also reported that same value of $R e_{o s c}$ was found with increasing or decreasing the velocity, which indicates $R e_{\text {osc }}$ has no hysteresis effect.

It was Camichel et al. [97] who first observed the initiation of the near-wake instability over $30 \leq R e \leq 60$. Secondary separations of the free shear layers were developed from the boundary of the near-wake with transverse displacement of the trail. These secondary separations stop the meeting of the free shear layers at the confluence point downstream. Coutanceau and Bouard [96] agreed that the onset of transverse oscillation of the nearwake indicates that the trail become unstable. For the sequences of $15 \leq R e \leq 20$, the elongated gathers at the end of the near-wake are longer than the shortened near-wake. The gathers are alternately formed on both sides of the near-wake [97]. However, there is another observation on this phenomenon. Taneda [12] wrote that "the gathers first appear from $R e>35$ near the downstream end of the near-wake border-line, move towards the rear end of the near-wake, tremble there for a while and die away." Taneda also stated that the sinusoidal oscillation of the trail takes place before the formation of the gathers and he believed the former induced the latter. Later, Gerrard [100] also observed the gathers appear periodically which produces a wavy trail. He pointed out the elongated twin bubbles become unstable and the appearance of the gathers carries away circulation into the wake. This is because the circulation within the bubbles is no longer balanced by diffusive transfer and it will be taken away from by the convective action of the gathers. According to Gerrard's work [100], both the spiky gather downstream and the one pointing into the bubble behind the body elongate in their directions respectively. The latter travels towards the body and a circulating motion is executed around the vortex attached to the body. The arm of the gather folds back towards the wake axis and this double layer oscillates but does not develop into a vortex street.

The oscillation is initiated by a noticeable change in pressure distribution. This adverse pressure gradient causes a separation and a characteristic inflection point to appear as reported by Coutanceau and Bouard [96]. This process was experimentally measured at $\mathrm{Re}=40$ by Grove et al. [105] and confirmed by experiments by Thom [104] and Homann [106]. 
Other than aforementioned, some puzzling discoveries on the near-wake instability were also reported. Thom [109] observed the oscillation of elastic wires with large amplitudes in water for Re far below $R e_{\text {osc }}$. Camichel and Teissié-Solier [98] triggered the near-wake instability at $R e<R e_{\text {osc }}$ by plucking the wire and later this was suppressed by viscous damping. The full sequence of the development of the wake were presented and the appearance of a steady closed near-wake behind a stationary cylinder at $R e=44$ was reported. The trail became undulating due to the impulse and gathers form alternately at the end of the near-wake. As the oscillation of the near-wake becomes settled, the waviness of the trail becomes weaker until the re-establishment of a stable and straight trail. Taneda [12] carried out similar experiments and measured the wave-length of trail oscillation $L_{w l}$ at a distance of $20 \mathrm{D}$ downstream of the cylinder, or non-dimensionally

$$
\frac{D}{L_{w l}}=f \times \frac{D}{U-u}
$$

where $u$ is the wave speed.

According to Taneda [12], the excitations are damped for $R e \leq 30$; the self-excited oscillations are sustained for $30<R e<45$; and for $R e>45$, the self-excitation oscillations form eddies along the trail.

\subsection{Decay of wake oscillations at low Re numbers}

In this section, we will discuss numerically the wake behind bluff bodies(circular cylinders) at Re low enough to have steady wake flow with the main characteristic of its oscillation. Presented here are the analysis of the numerical results that demonstrate the wake oscillation at low Re range of 30-44, which is the oscillatory regime right before the onset of vortex shedding for circular cylinders. We will also introduce a theoretical mode resembling a oscillator to explain the decay of wake oscillation. A novel low-dimensional model is introduced to describe the formation, development and decay of the closed near-wake at low Re. Later, the St-Re relationship of Re 16-47 compared to Camichel's experiments will discussed. 

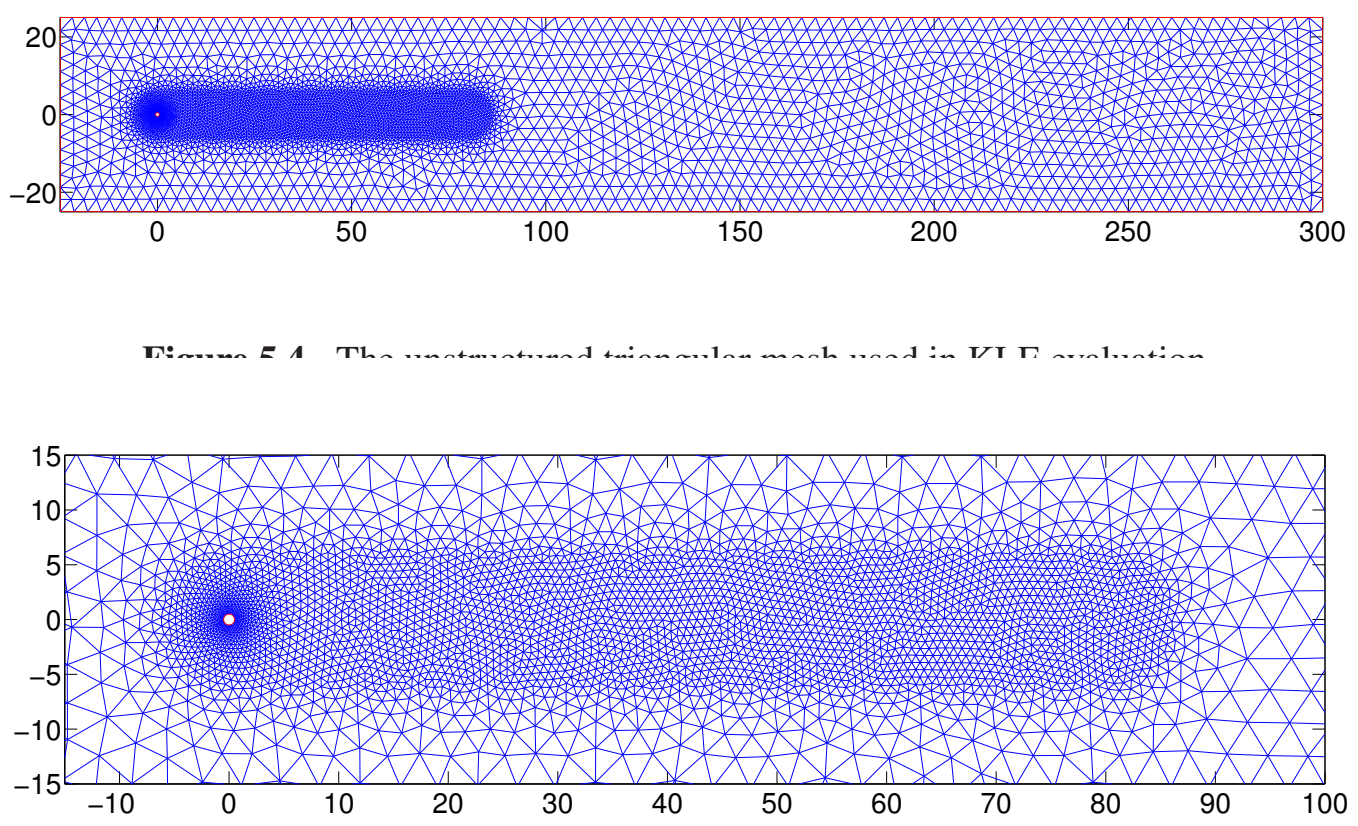

Figure 5.5. The closer view of the mesh above.

\subsubsection{Numerical results}

The velocity and vorticity field was solved with a steady two-dimensional flow around a circular cylinder by applying the aforementioned KLE method. The experiments were carried out on unstructured meshes of different widths $(15 D-50 D$, spaced very $5 D)$ in order to exclude the blockage effect because of the boundary of the flow domain(size of the meshes). The mesh of 25D width is shown in Figure 5.4. It is an unstructured triangular mesh with 4633 nodes and 9028 triangles. Figure 5.5 gives a closer view of the denser plateau with two "cones". The front cone is centred at the origin and the rear cone is centred at $80 \mathrm{D}$. This is the reason why 80 seconds was chosen as the period of the perturbation in the velocity boundary condition. In such case, we can perform the evaluation of the vorticity on a denser region that plays a significant role in the wake development and propagation while limiting computational cost, especially for low Re cases. The numerical study of blockage effects remains unsolved, which could be more important as the Re goes to the lower end of the range.

When the flow first passes around the cylinder, it introduces a sudden change in the velocity, thus vorticity, in the vicinity of the cylinder during the generation of the wake. To diminish the abrupt disturbance produced and get a more stable field of vorticity, a modified velocity boundary condition was developed. That is, once the velocity of the free uniform flow increase from 0 to 1 in a very short time, small perturbations of certain percentile were 


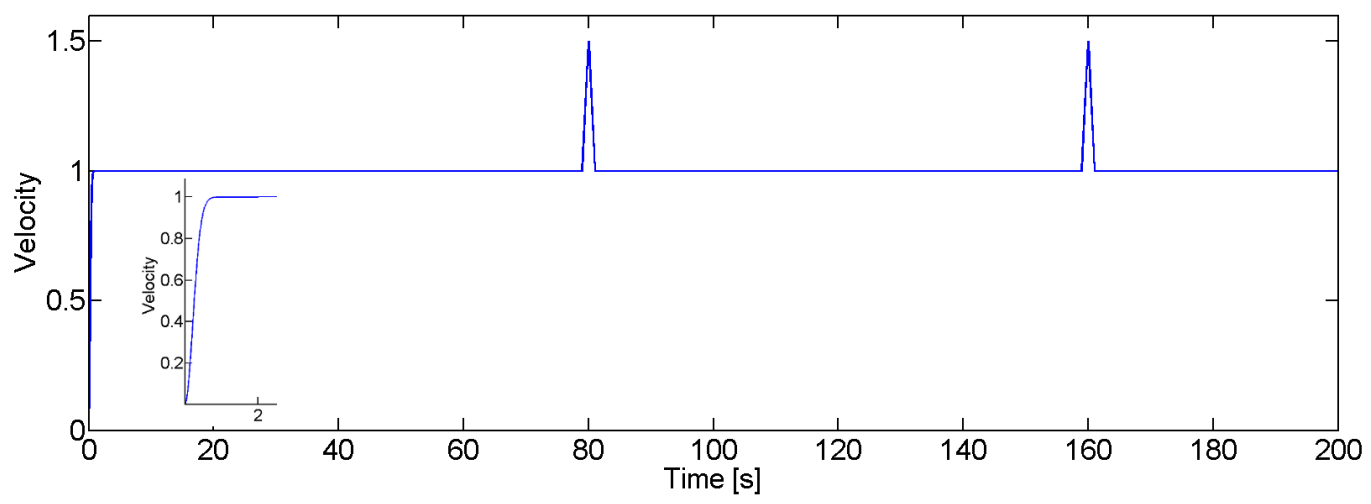

Figure 5.6. The modified velocity boundary profile.

imposed on the original value every 80 seconds, such as 1\%,5\%,10\% and 50\% increase in the velocity boundary condition. The modified velocity boundary conditions with $1 \%, 5 \%$, $10 \%$ increment are relatively weak when interacting with the wake from the previous period. The resulting blended wake tends to damp itself quickly to what it was produced by the uniform free flow. Thus, in order to obtain a well-defined and steady blended wake, some other perturbations have been tested. It was found that the one with $50 \%$ increment gives better results. All the numerical results presented in this chapter is based on this $50 \%$ increment in velocity, see its profile in Figure 5.6. Here, a word "Event" is used to described the wake propagation of the disturbed flow downstream for every complete period. For instance, the wake propagation in the course of the first 80 seconds is named "First-Event", "Second-Event" for the second 80 seconds, and so on.

Every time when the perturbation is applied, the blending of the previous wake and the initiation of the wake resulting from the perturbation at the alternation of the previous event and the current event needs some time to become settled and stabilized. This is very important to be noted when post-processing analysis was performed. As shown in the mesh, it is 300 diameters into the downstream which gives some limitation on how long we can actually run the experiment with the concern that the wake would reach the rear boundary of the computational domain creating interference and transferring back to the wake. This means, in order to ensure the accuracy, the runs need to be stopped before the wake reach the end of the mesh. Hence, with 80 seconds for an event, 200 seconds for the whole run is on the safe side. Discarding the first event, and the incomplete third event on the vorticity field, a proper "time window" on the second event was chosen to analyse the rate of decay and the frequency. The time window is confined within time span in the course of the second event.

In the experiments, the vorticity is computed at three different locations with numerical "probes" at $1.3 \mathrm{D}, 16 \mathrm{D}, 28 \mathrm{D}(\mathrm{D}$, the diameter of the cylinder $)$ downstream. As shown in 
Figure 5.7 through Figure 5.21, it is clear that the wake oscillations fall into a sinusoidal pattern "bounded" by two exponential decaying lines defined by the peaks values of the vorticity. The oscillations at three locations for a specified Re show consistency in physical characteristics. The oscillations are damping out as time goes by with different decay and they oscillate under similar frequency. As Re increases, the viscous effect becomes weaker, and the oscillation tends to damp slower and survives further with time. 


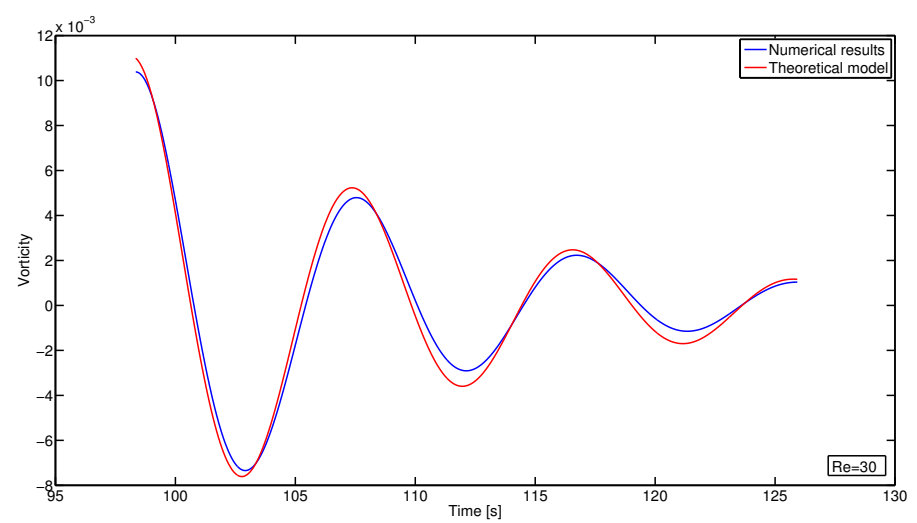

(a)

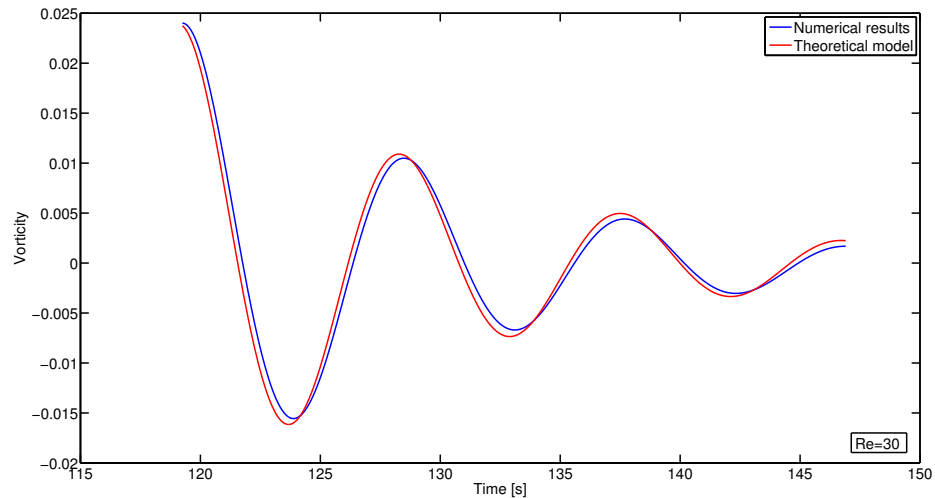

(b)

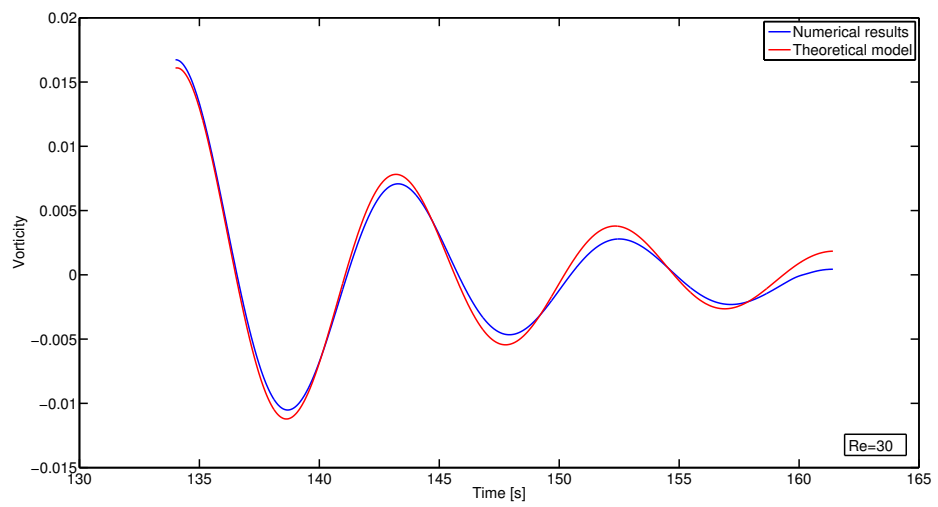

(c)

Figure 5.7. Comparison of numerical results and theoretical model for wake oscillation at $\mathrm{Re}=30$ : $(a)$, probe locates at 1.3(cylinder diameter) downstream; $(b)$, probe locates at 16(cylinder diameter) downstream; $(c)$, probe locates at 28(cylinder diameter) downstream. 


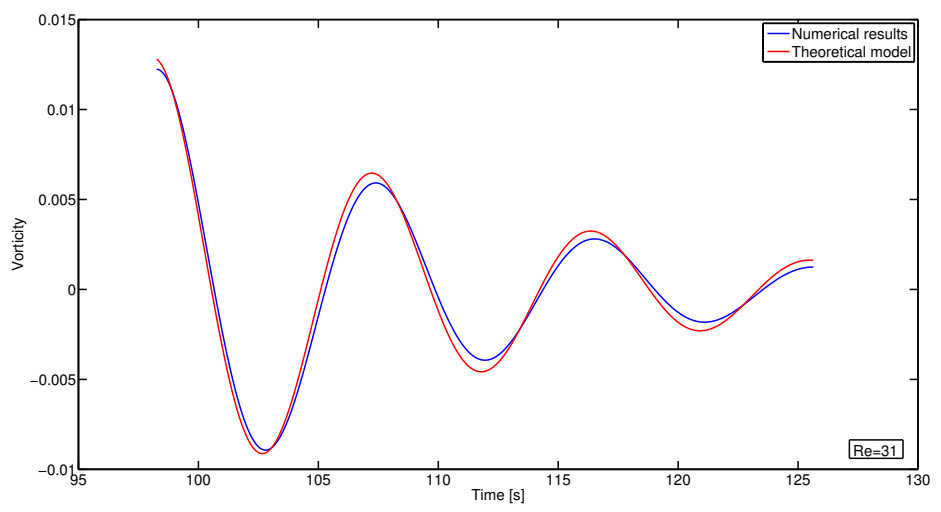

(a)

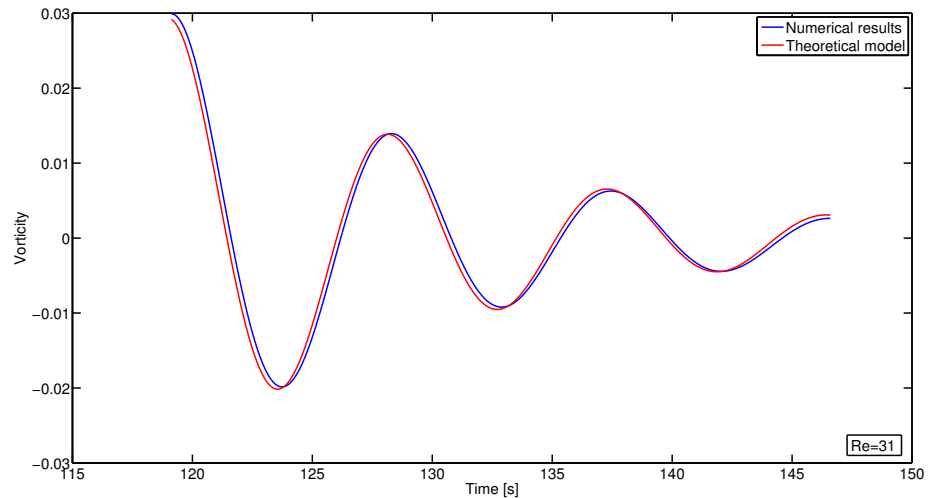

(b)

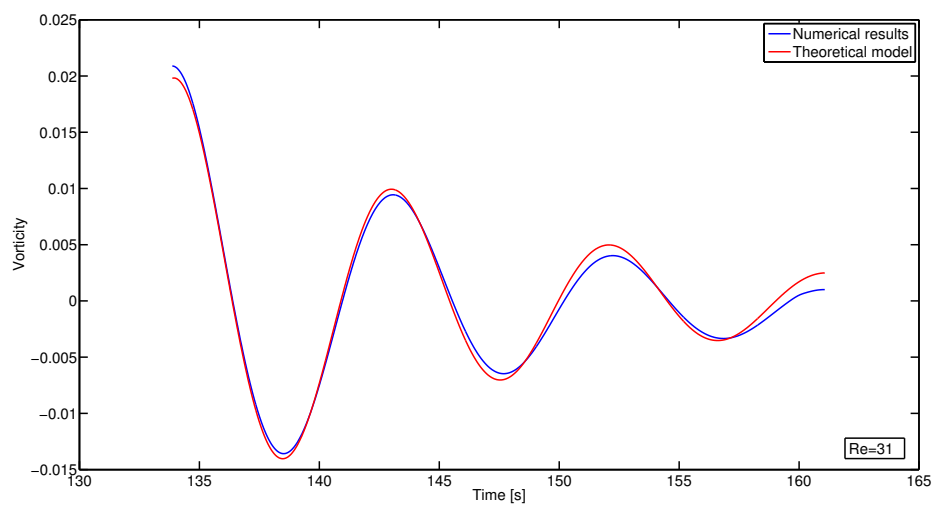

(c)

Figure 5.8. Comparison of numerical results and theoretical model for wake oscillation at $\mathrm{Re}=31$ : $(a)$, probe locates at 1.3(cylinder diameter) downstream; $(b)$, probe locates at 16(cylinder diameter) downstream; $(c)$, probe locates at 28(cylinder diameter) downstream. 


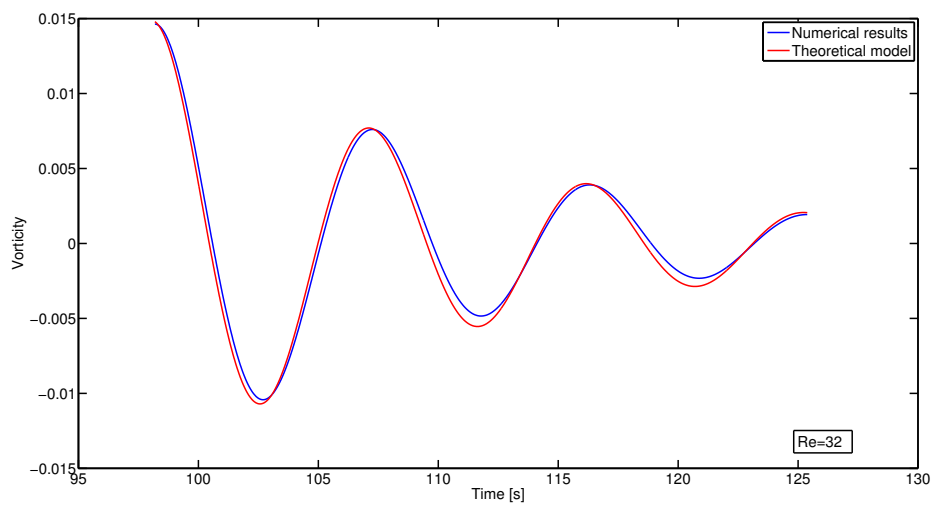

(a)

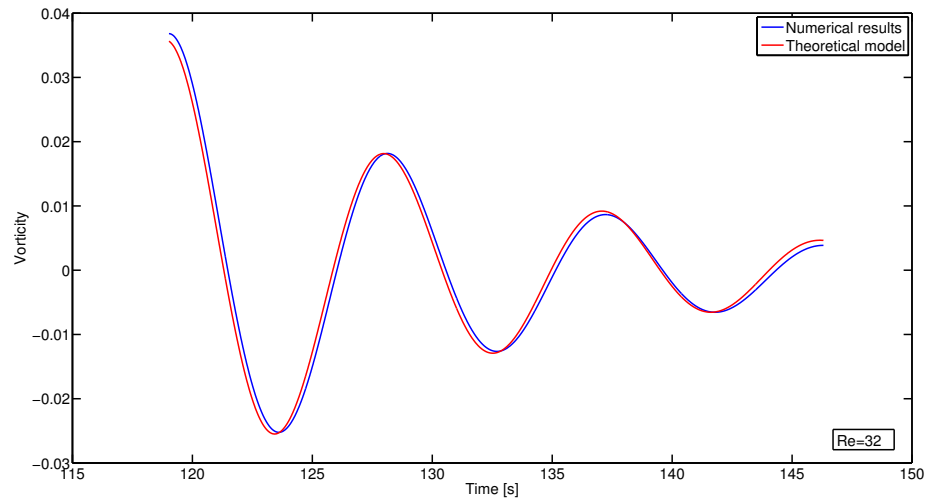

(b)

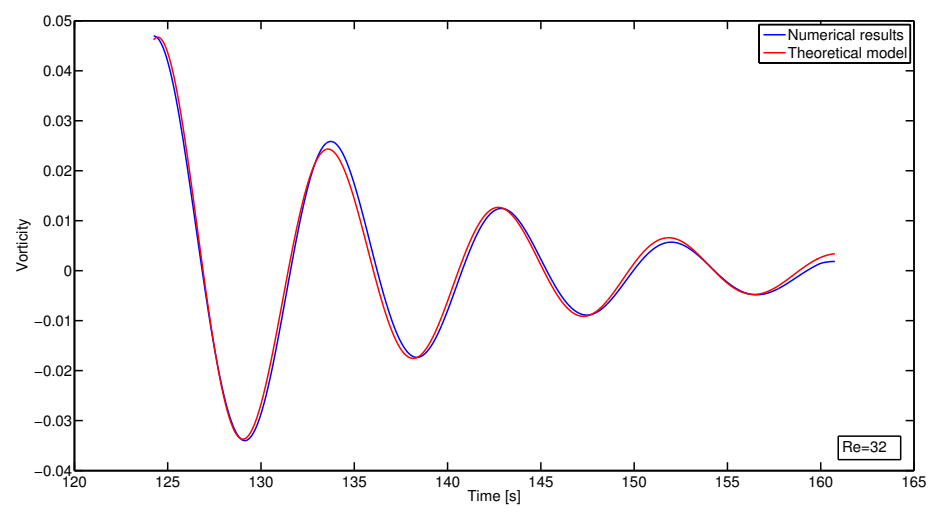

(c)

Figure 5.9. Comparison of numerical results and theoretical model for wake oscillation at $\operatorname{Re}=32$ : $(a)$, probe locates at 1.3(cylinder diameter) downstream; $(b)$, probe locates at 16(cylinder diameter) downstream; $(c)$, probe locates at 28(cylinder diameter) downstream. 


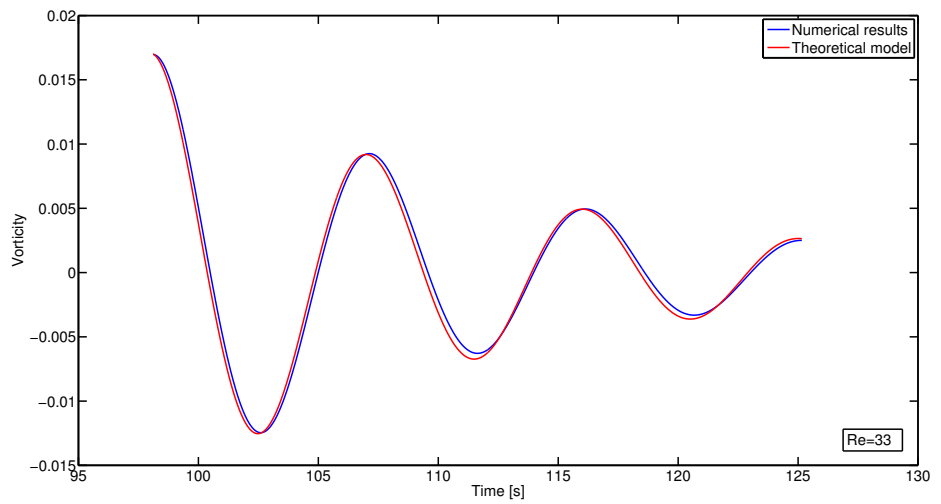

(a)

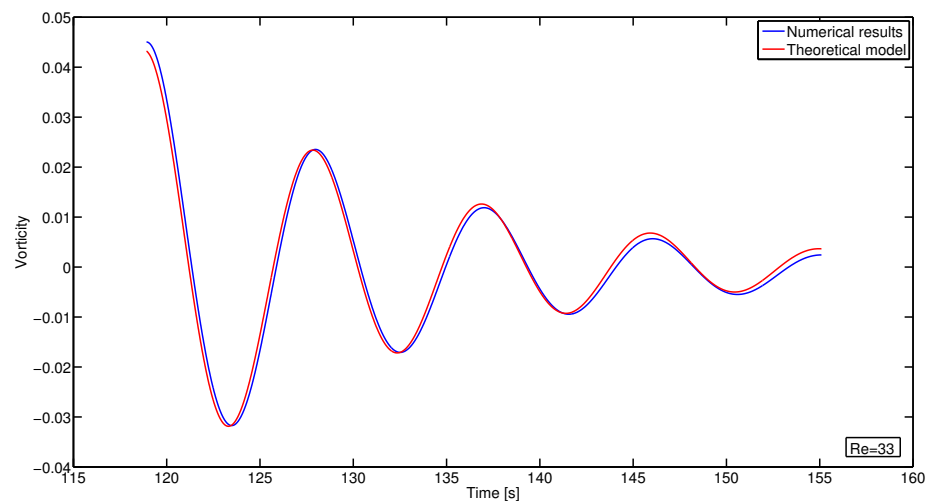

(b)

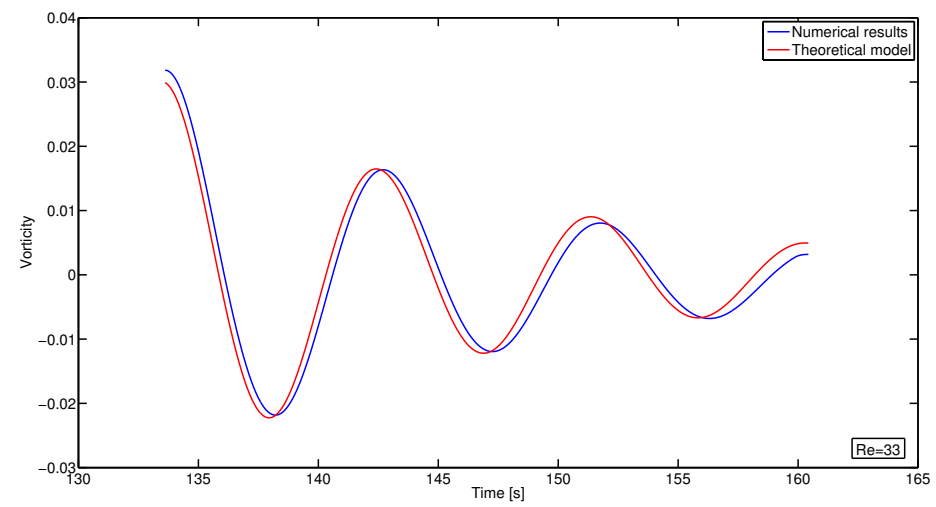

(c)

Figure 5.10. Comparison of numerical results and theoretical model for wake oscillation at $\mathrm{Re}=33$ : $(a)$, probe locates at 1.3(cylinder diameter) downstream; $(b)$, probe locates at 16(cylinder diameter) downstream; $(c)$, probe locates at 28(cylinder diameter) downstream. 


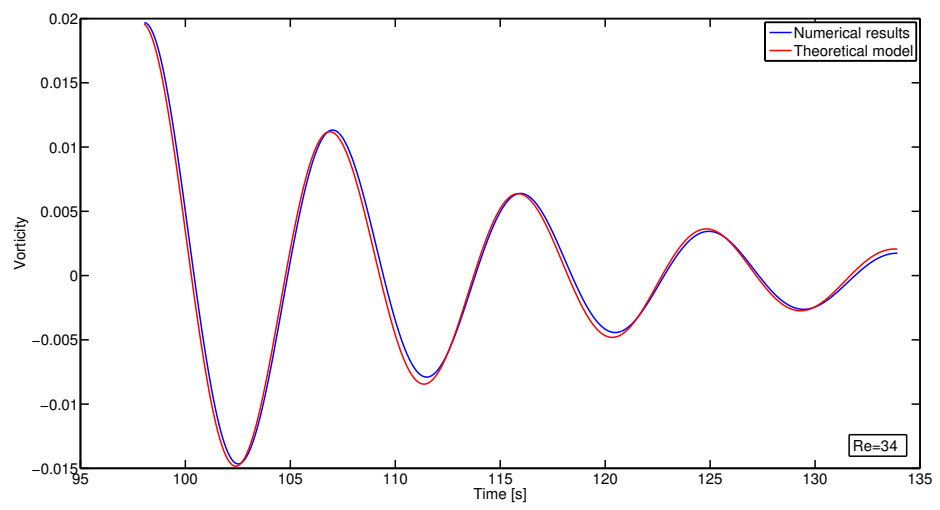

(a)

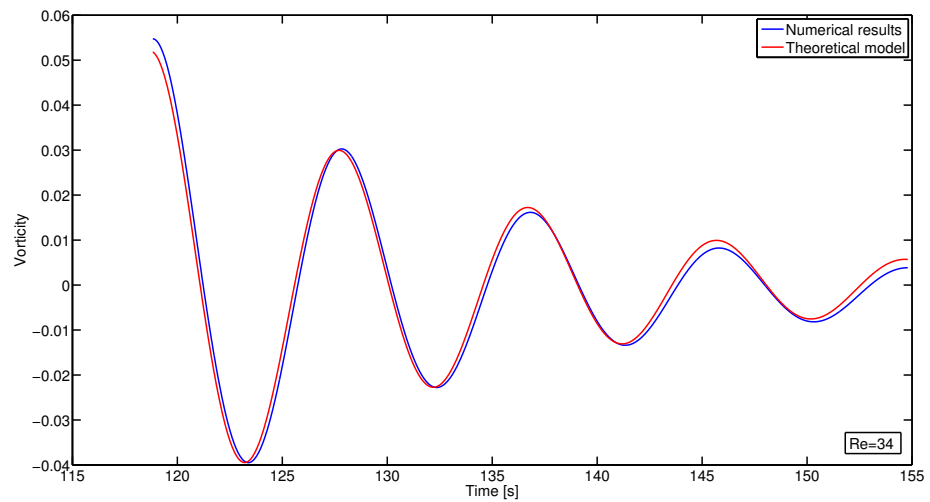

(b)

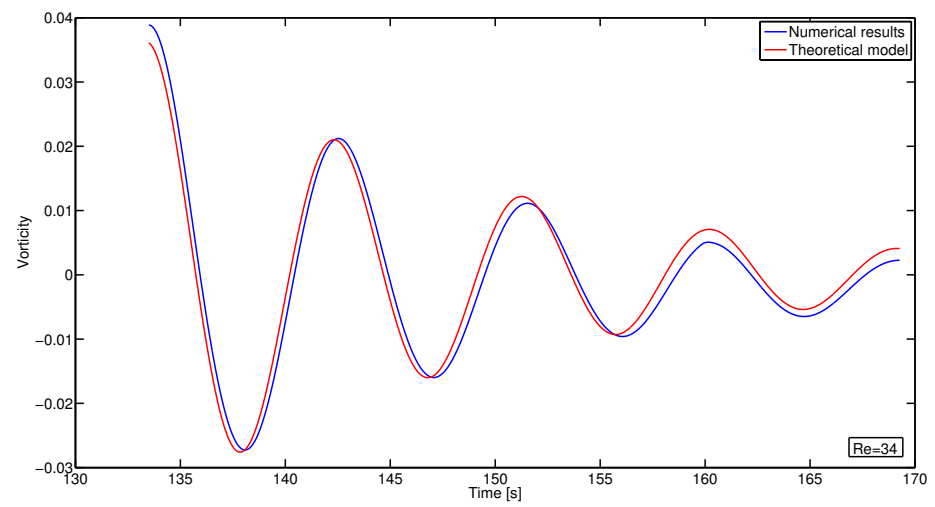

(c)

Figure 5.11. Comparison of numerical results and theoretical model for wake oscillation at $\mathrm{Re}=34$ : (a), probe locates at 1.3(cylinder diameter) downstream; $(b)$, probe locates at 16(cylinder diameter) downstream; $(c)$, probe locates at 28(cylinder diameter) downstream. 


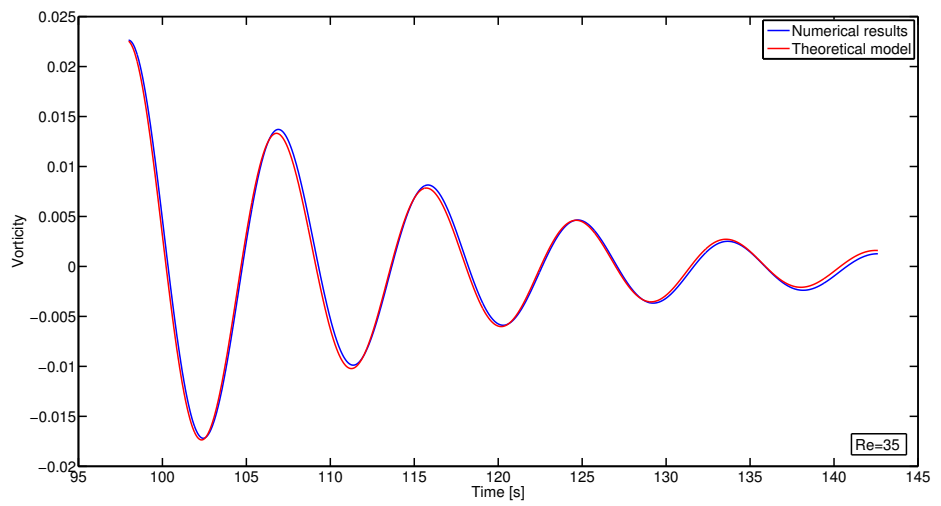

(a)

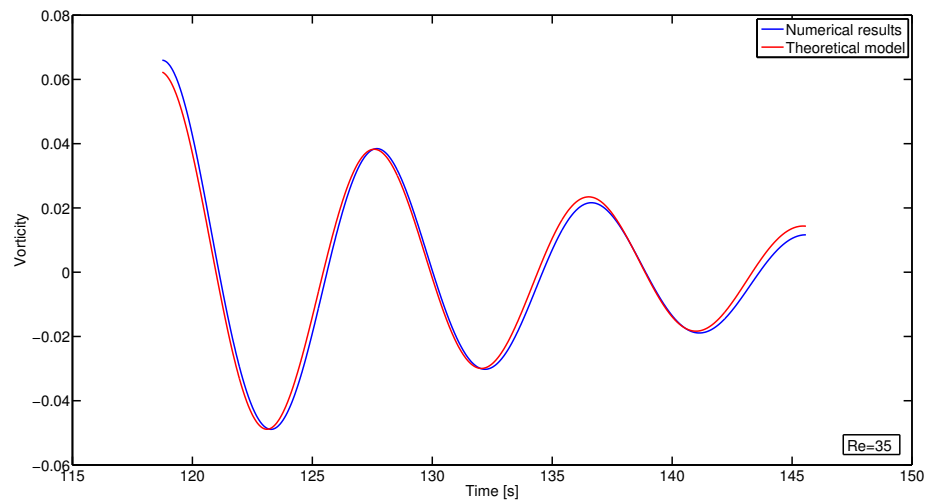

(b)

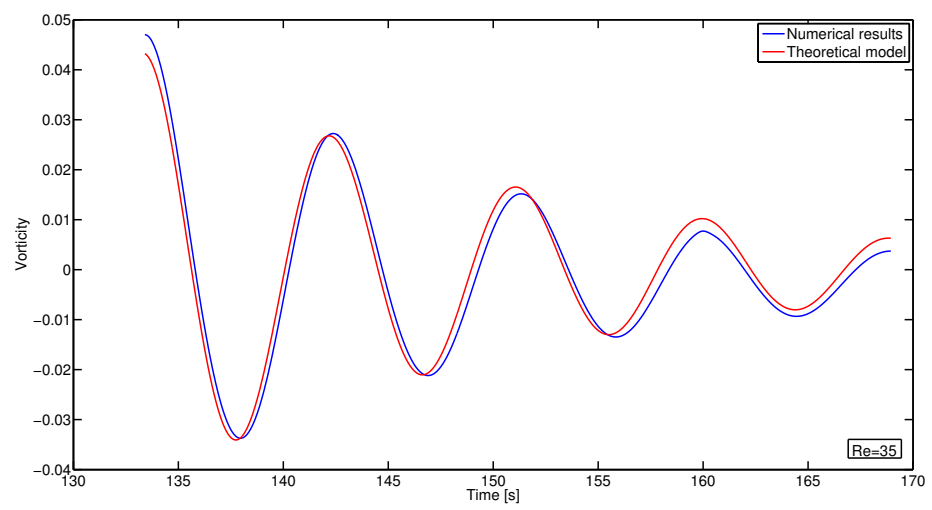

(c)

Figure 5.12. Comparison of numerical results and theoretical model for wake oscillation at $\mathrm{Re}=35$ : (a), probe locates at 1.3(cylinder diameter) downstream; $(b)$, probe locates at 16(cylinder diameter) downstream; $(c)$, probe locates at 28(cylinder diameter) downstream. 


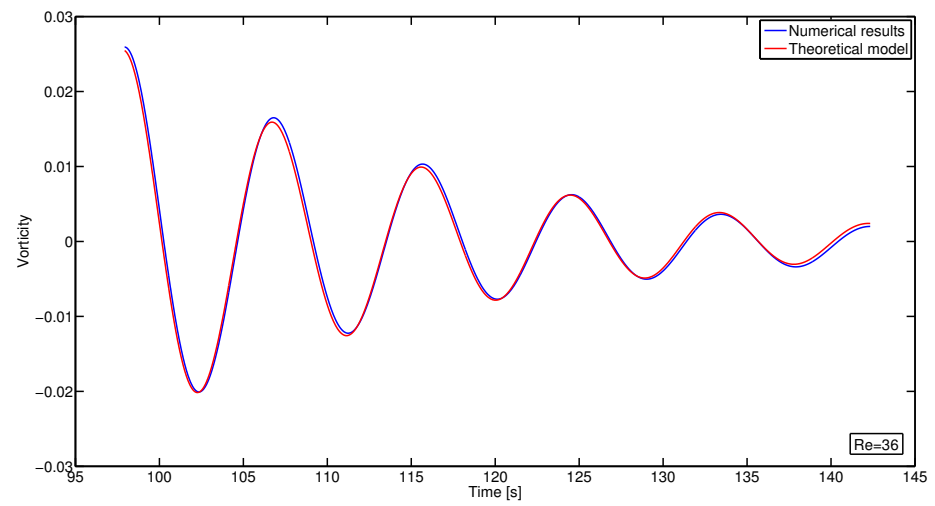

(a)

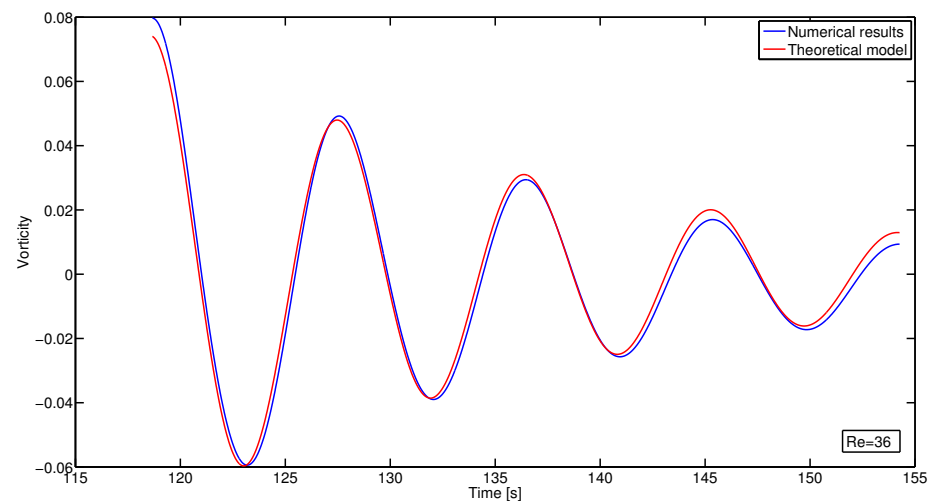

(b)

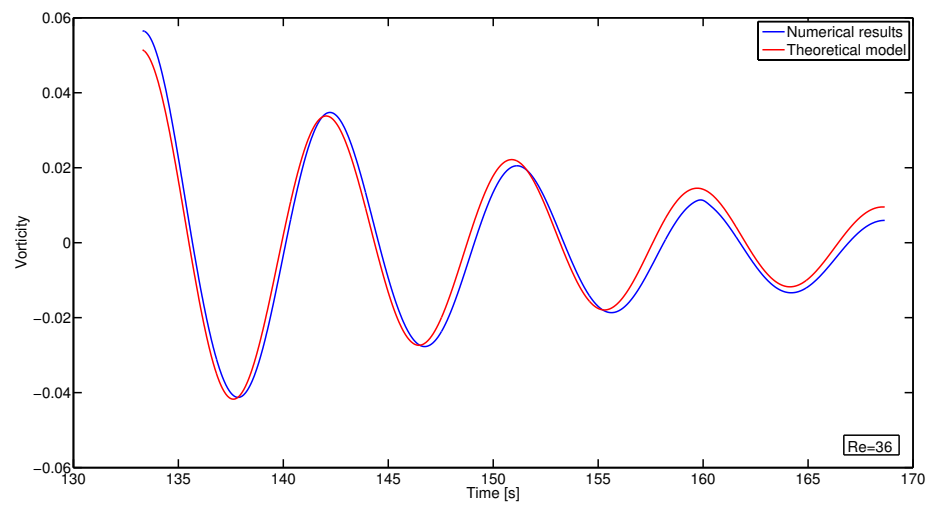

(c)

Figure 5.13. Comparison of numerical results and theoretical model for wake oscillation at $\mathrm{Re}=36$ : (a), probe locates at 1.3(cylinder diameter) downstream; $(b)$, probe locates at 16(cylinder diameter) downstream; (c), probe locates at 28(cylinder diameter) downstream. 


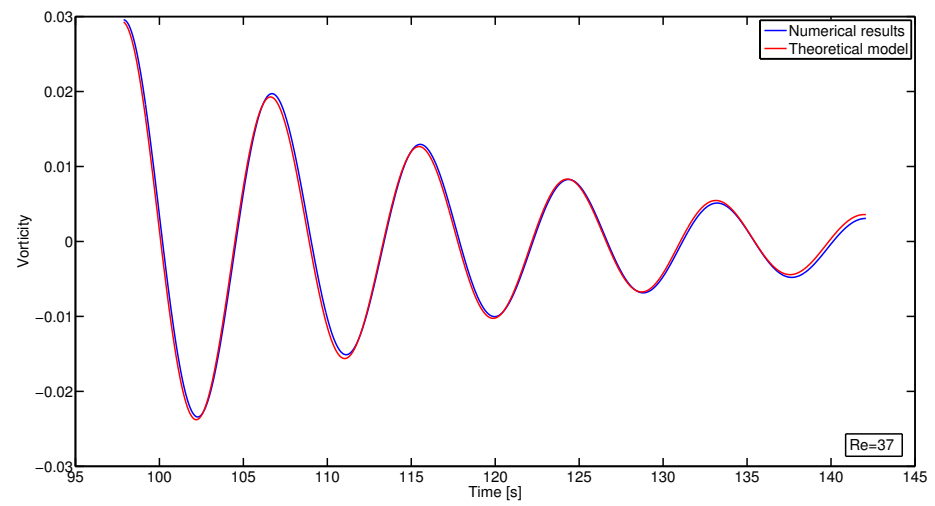

(a)

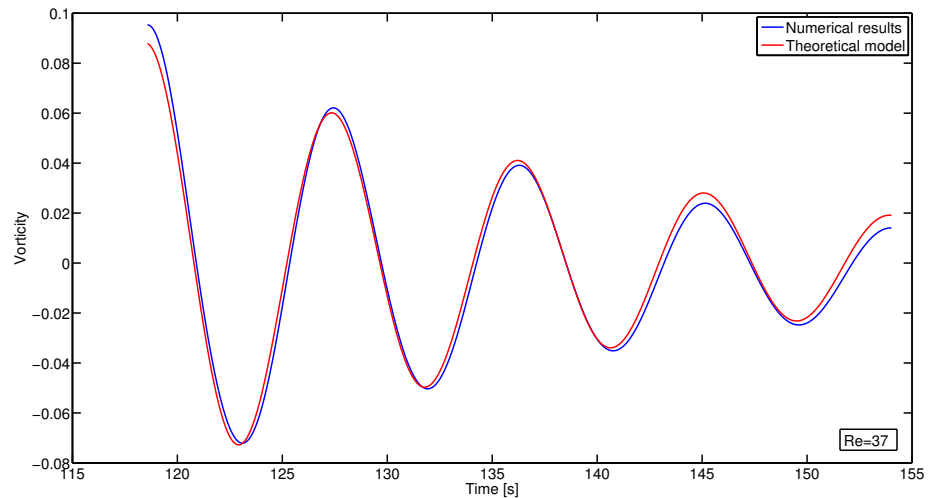

(b)

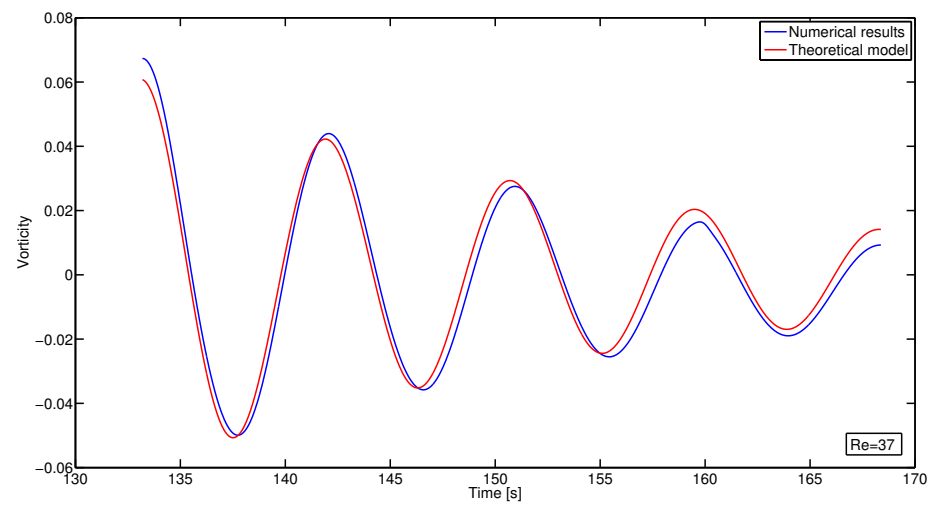

(c)

Figure 5.14. Comparison of numerical results and theoretical model for wake oscillation at $\mathrm{Re}=37$ : $(a)$, probe locates at 1.3(cylinder diameter) downstream; $(b)$, probe locates at 16(cylinder diameter) downstream; $(c)$, probe locates at 28(cylinder diameter) downstream. 


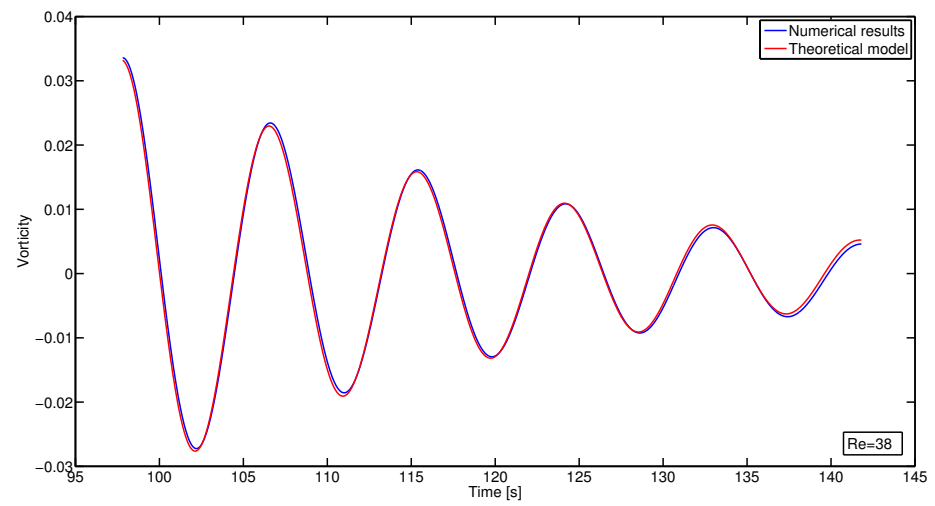

(a)

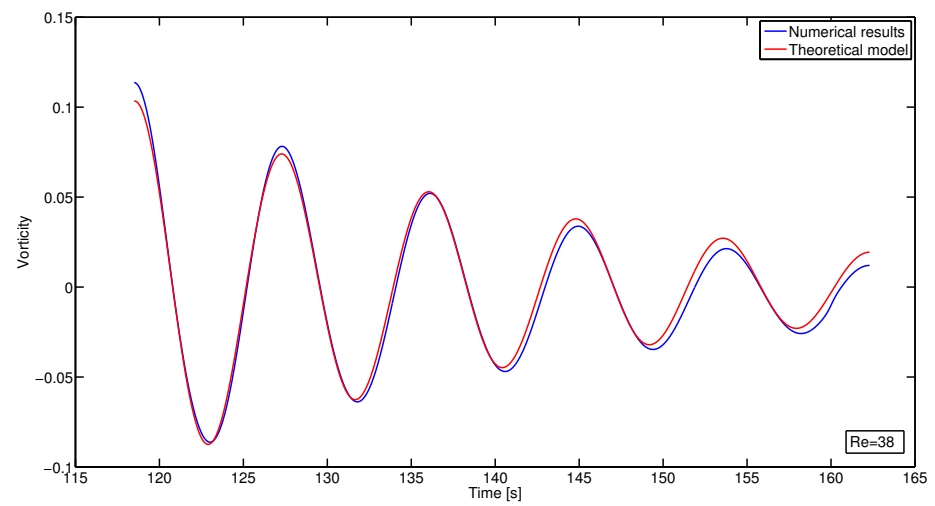

(b)

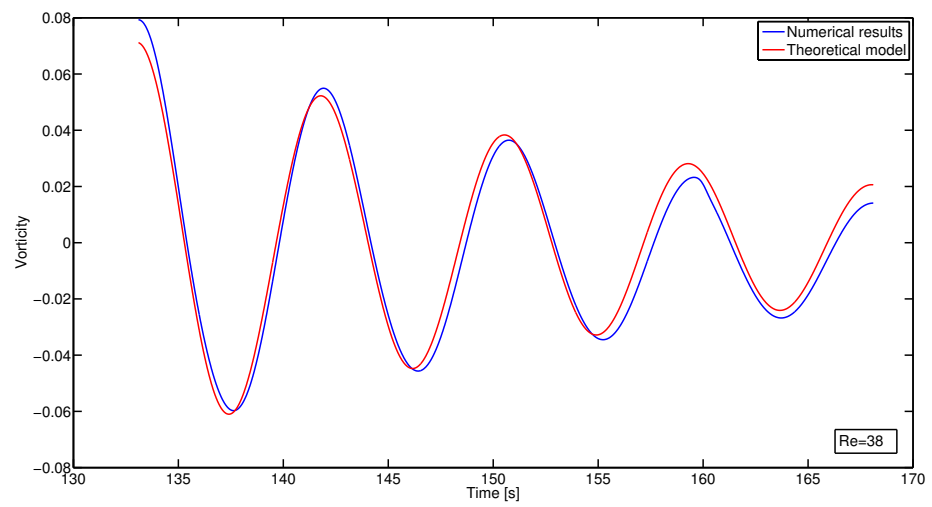

(c)

Figure 5.15. Comparison of numerical results and theoretical model for wake oscillation at $\mathrm{Re}=38$ : (a), probe locates at 1.3(cylinder diameter) downstream; $(b)$, probe locates at 16(cylinder diameter) downstream; (c), probe locates at 28(cylinder diameter) downstream. 


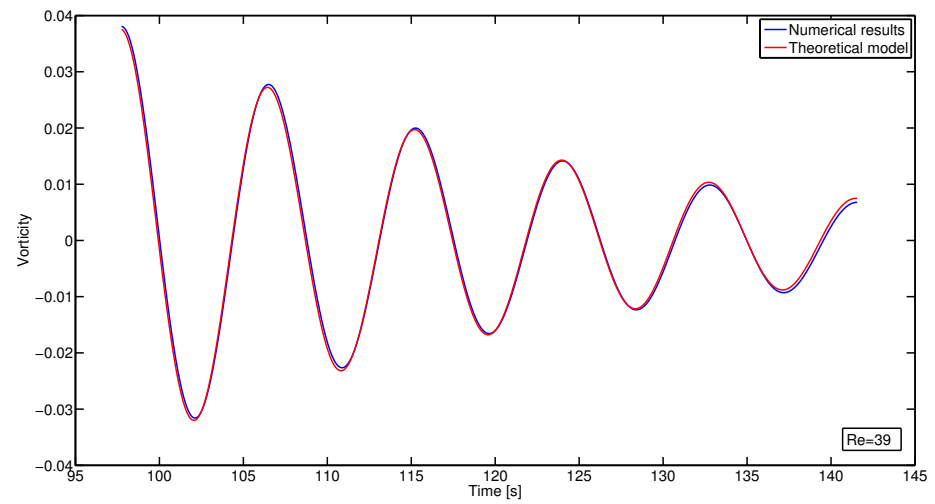

(a)

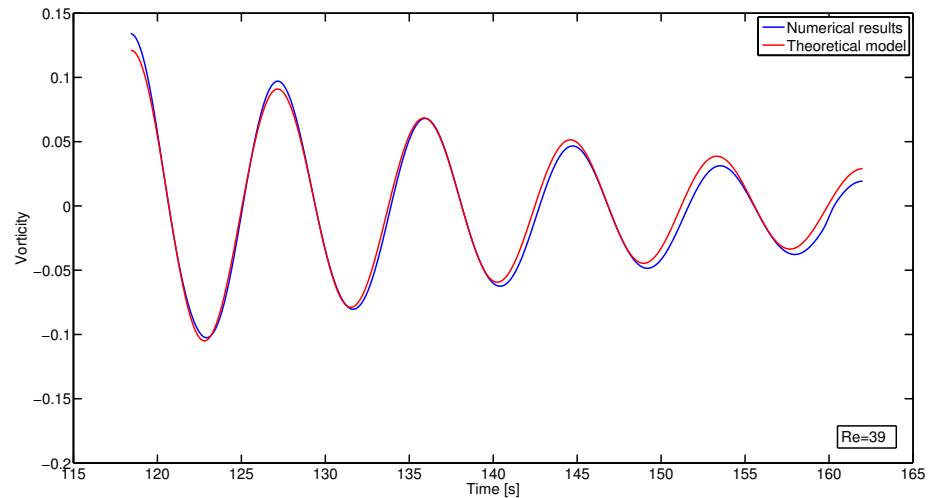

(b)

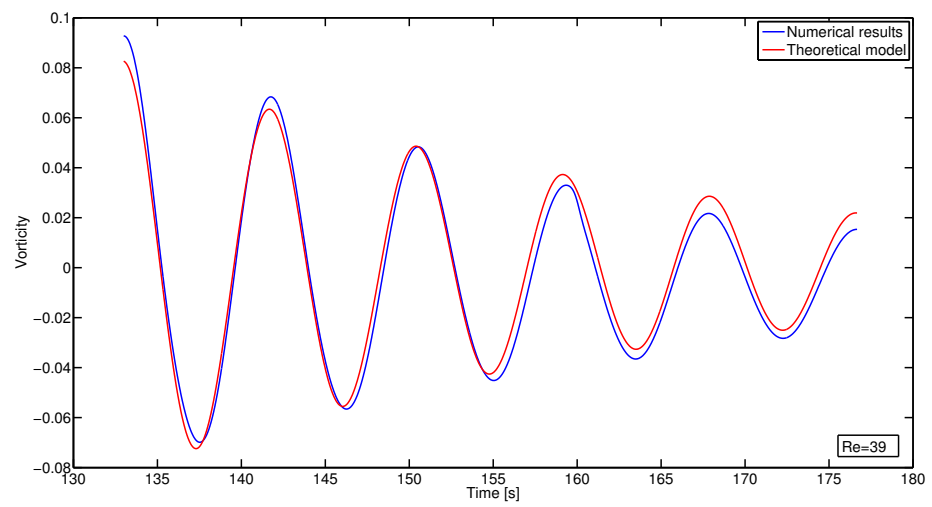

(c)

Figure 5.16. Comparison of numerical results and theoretical model for wake oscillation at $\mathrm{Re}=39$ : (a), probe locates at 1.3(cylinder diameter) downstream; $(b)$, probe locates at 16(cylinder diameter) downstream; (c), probe locates at 28(cylinder diameter) downstream. 


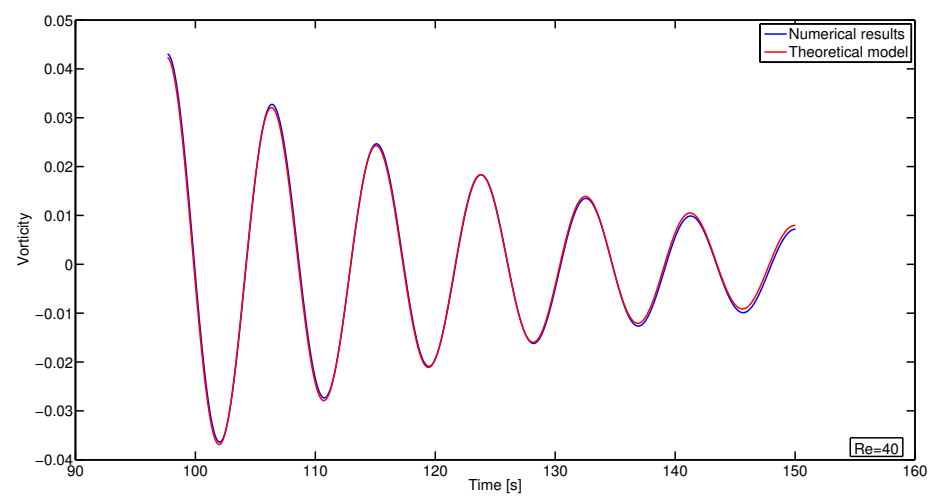

(a)

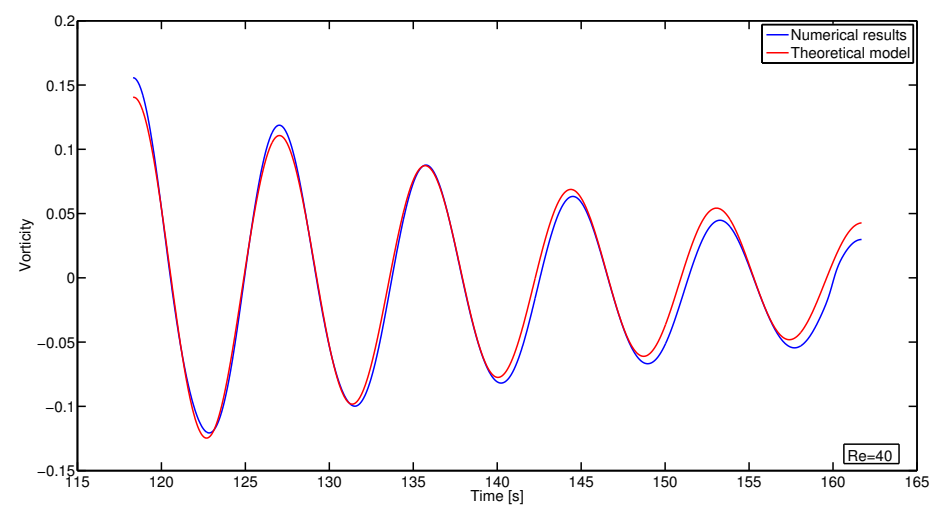

(b)

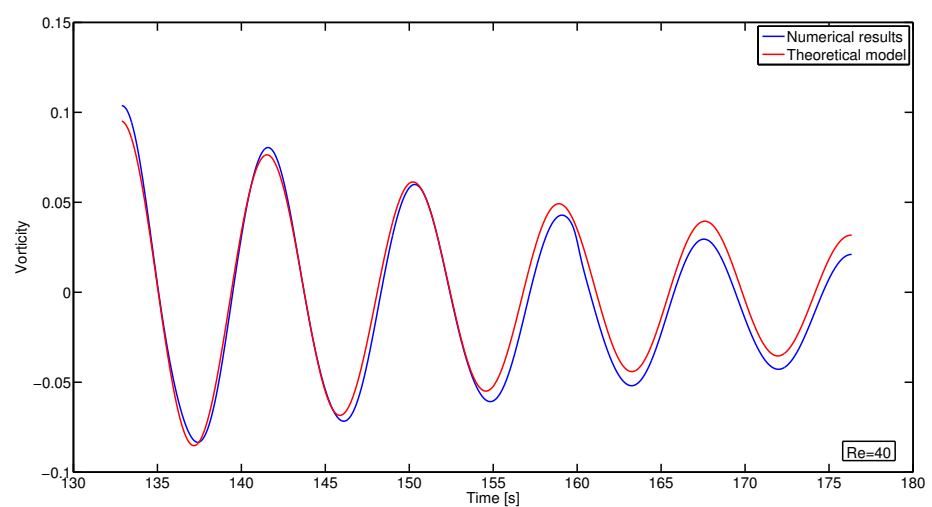

(c)

Figure 5.17. Comparison of numerical results and theoretical model for wake oscillation at $\operatorname{Re}=40$ : $(a)$, probe locates at 1.3(cylinder diameter) downstream; $(b)$, probe locates at 16(cylinder diameter) downstream; $(c)$, probe locates at 28(cylinder diameter) downstream. 


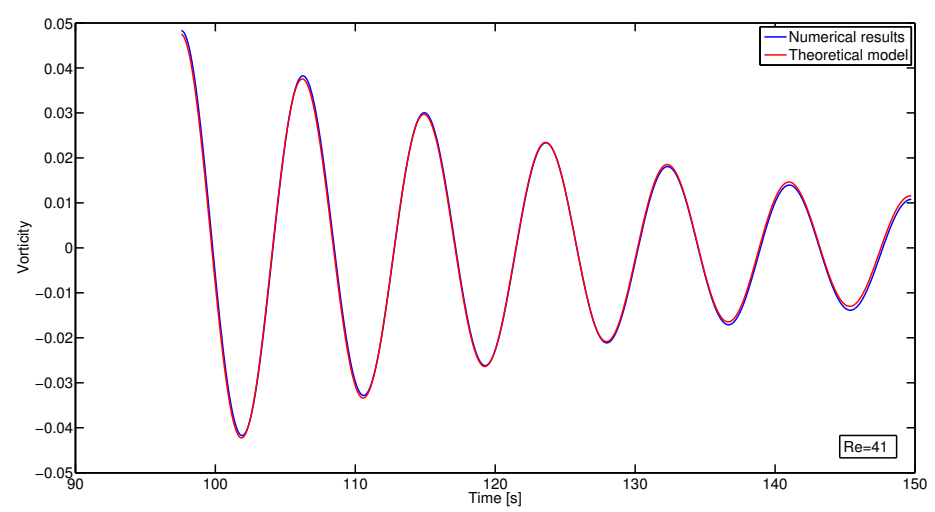

(a)

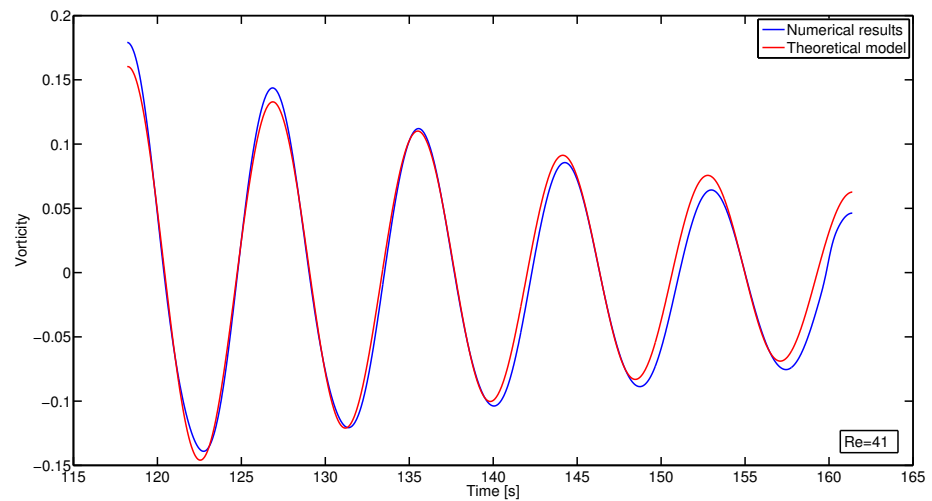

(b)

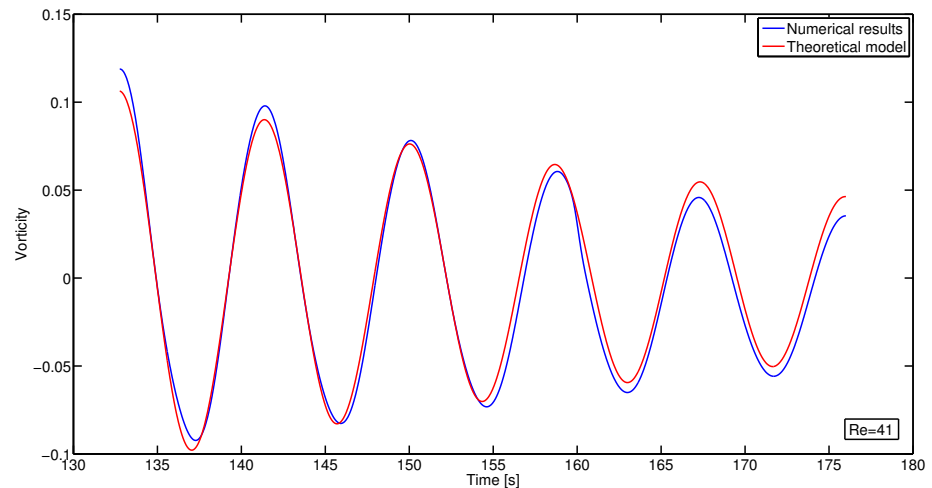

(c)

Figure 5.18. Comparison of numerical results and theoretical model for wake oscillation at $\mathrm{Re}=41$ : $(a)$, probe locates at 1.3(cylinder diameter) downstream; $(b)$, probe locates at 16(cylinder diameter) downstream; $(c)$, probe locates at 28(cylinder diameter) downstream. 


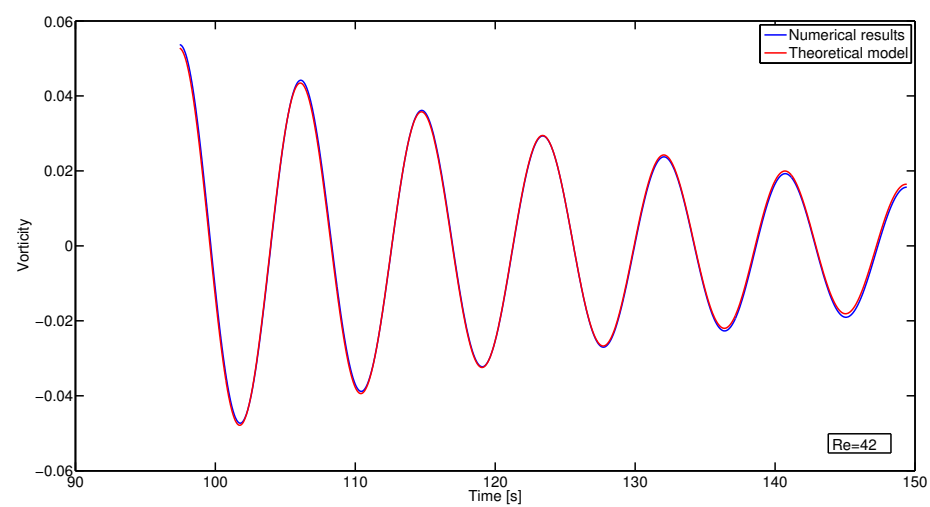

(a)

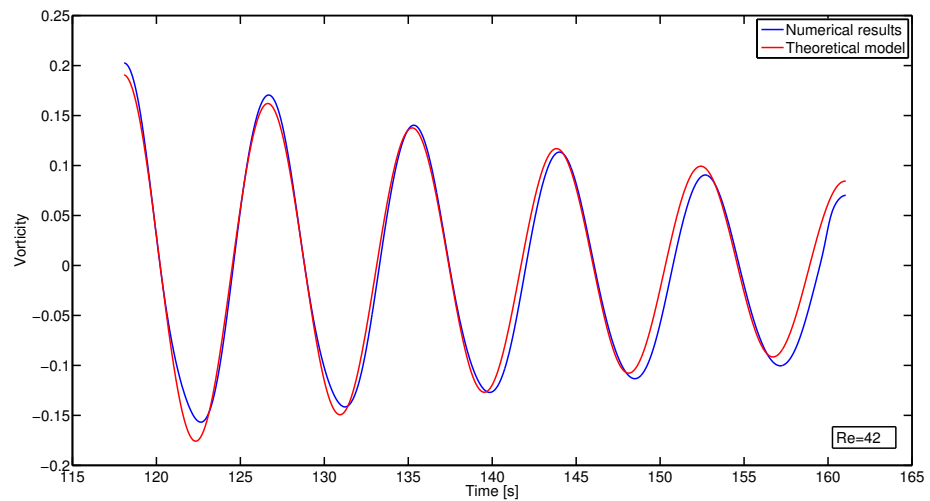

(b)

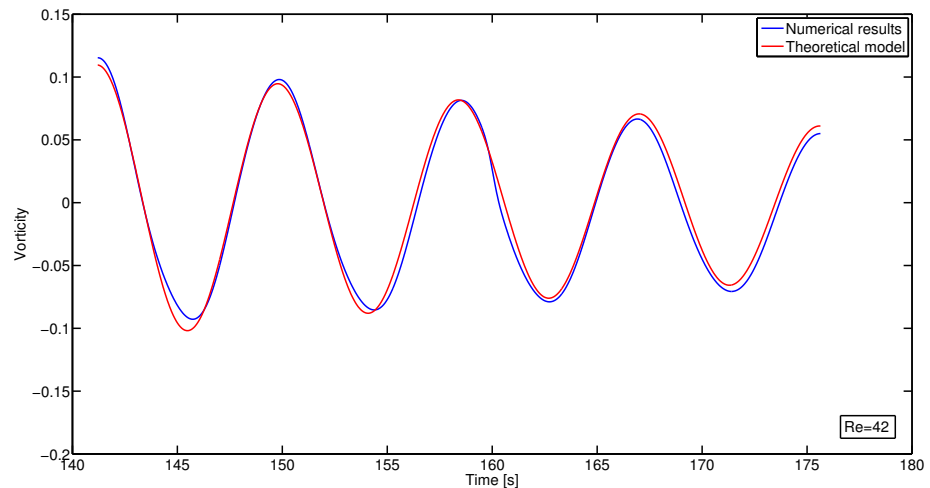

(c)

Figure 5.19. Comparison of numerical results and theoretical model for wake oscillation at $\mathrm{Re}=42$ : $(a)$, probe locates at 1.3(cylinder diameter) downstream; $(b)$, probe locates at 16(cylinder diameter) downstream; $(c)$, probe locates at 28(cylinder diameter) downstream. 


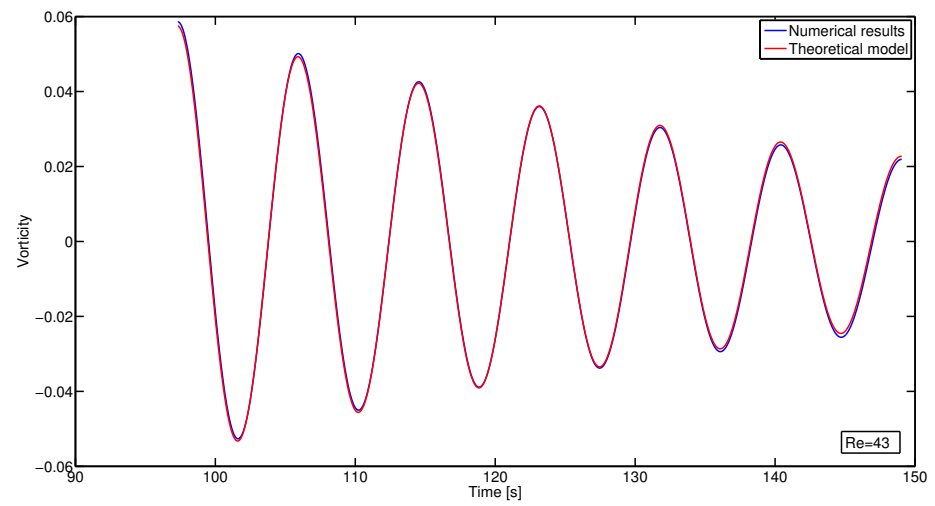

(a)

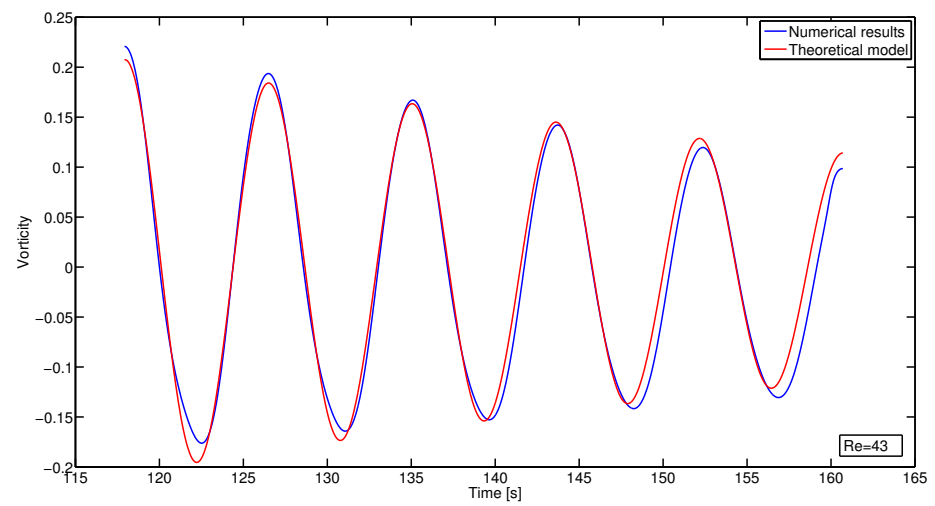

(b)

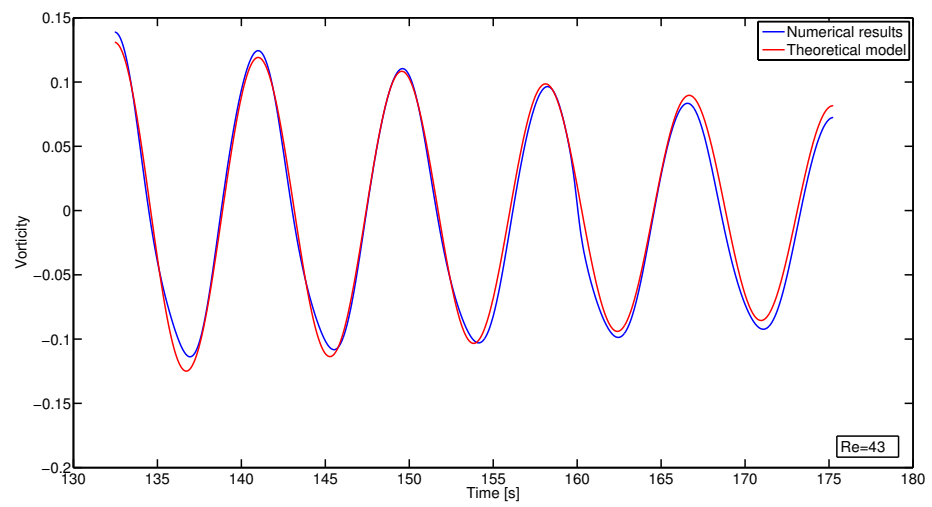

(c)

Figure 5.20. Comparison of numerical results and theoretical model for wake oscillation at $\operatorname{Re}=43$ : $(a)$, probe locates at 1.3(cylinder diameter) downstream; $(b)$, probe locates at 16(cylinder diameter) downstream; $(c)$, probe locates at 28(cylinder diameter) downstream. 


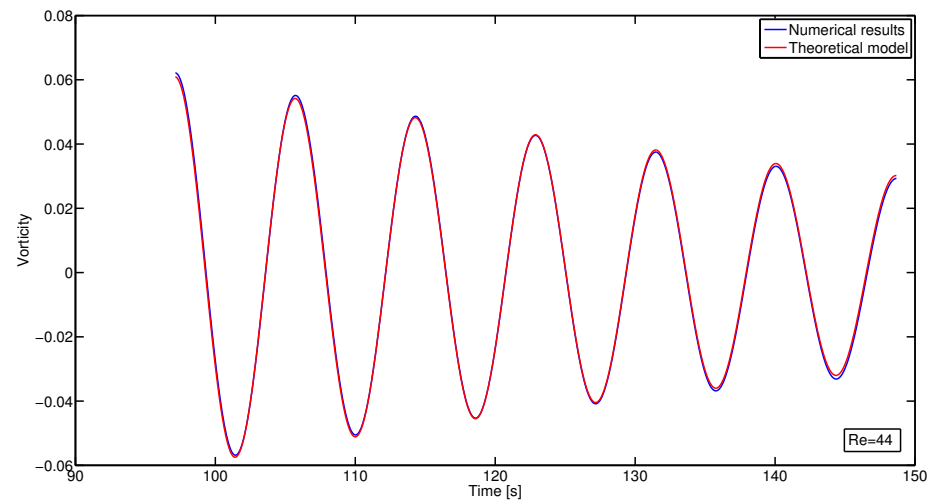

(a)

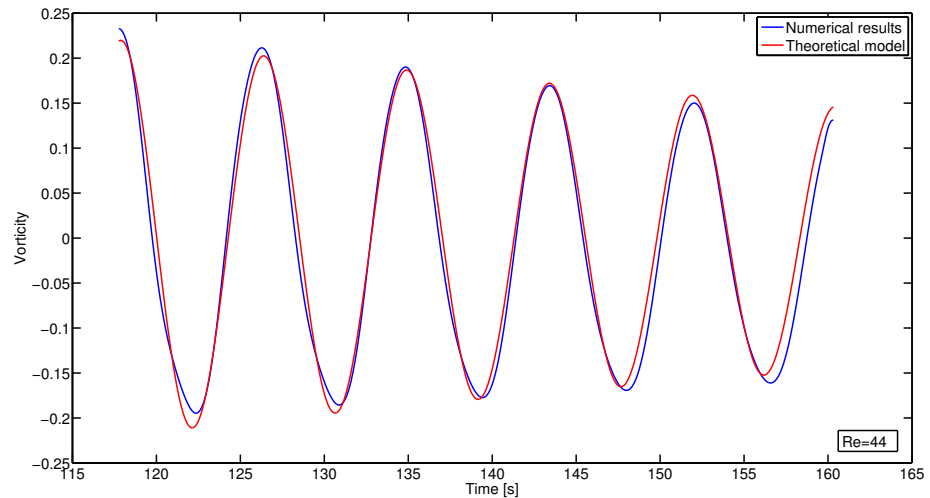

(b)

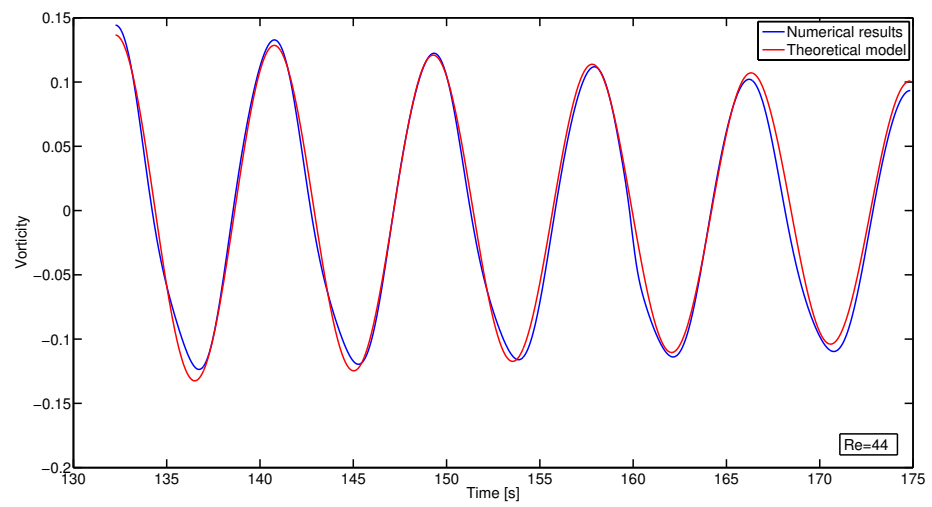

(c)

Figure 5.21. Comparison of numerical results and theoretical model for wake oscillation at $\mathrm{Re}=44$ : $(a)$, probe locates at 1.3(cylinder diameter) downstream; $(b)$, probe locates at 16(cylinder diameter) downstream; $(c)$, probe locates at 28(cylinder diameter) downstream. 
To analyse this phenomenon quantitatively, a theoretical model was proposed which will be described as oscillations with exponential amplitude. The theoretical model describes the time evolution of the amplitude of the vorticity fluctuations on the twin vortex wake. The theoretical results accurately match the numerical results in terms of the frequency of the oscillation and rate of decay as shown in Equation 5.3.

$$
\omega_{a n}=B \times \exp \left(D_{W} \times T_{t}+i \times \frac{2 \times \pi}{T_{\text {ave }}} \times\left(T_{t}-\text { const }\right)\right)
$$

where $\omega_{a n}$ is the theoretical vorticity, $B$ is the amplitude of the wake oscillation, $D_{W}$ is the decay of the wake oscillation, $T_{t}$ is the time span in which the wake oscillates, $T_{\text {ave }}$ is the average frequency of the wake oscillation, const is a constant.

$B$, see table 5.1, describes the amplitude of the oscillations in the wake in the theorical model. It varies from case to case, even differs at different points probed for the same $\mathrm{Re}$ number. It could be related to the amount of accumulated vorticity when the perturbation triggered because viscous effect in the diffusion of vorticity dominates in the flow at low Re.

Table 5.1

Amplitude $B$ at different probes locations $x$

\begin{tabular}{cccc}
\hline$R e$ & $x=1.3$ & $x=16$ & $x=28$ \\
\hline 30 & 33.41940439 & 625.8083656 & 654.2342962 \\
31 & 21.48207125 & 506.7704403 & 541.0094479 \\
32 & 18.48217369 & 260.5465109 & 344.5422445 \\
33 & 14.73228879 & 145.5609316 & 238.0137998 \\
34 & 9.232344941 & 76.29319975 & 122.6927107 \\
35 & 7.57326927 & 41.56710986 & 59.99637707 \\
36 & 4.666406929 & 24.84206273 & 29.85190741 \\
37 & 3.069072808 & 14.46383661 & 15.24253645 \\
38 & 2.038386928 & 9.573784102 & 7.915212202 \\
39 & 1.37479717 & 5.83624069 & 4.713889986 \\
40 & 0.961566957 & 3.609322955 & 2.738504395 \\
41 & 0.668891745 & 2.095127199 & 1.364125795 \\
42 & 0.469752514 & 1.79467465 & 1.20628421 \\
43 & 0.330397295 & 1.076437968 & 0.567538207 \\
44 & 0.228914681 & 0.678878196 & 0.350339504 \\
\hline
\end{tabular}

This aforementioned function is a product of a decaying exponential and a sine or cosine, in which the envelops of the oscillations are given by exponential decaying. $\tau=-1 / D_{W}$ 


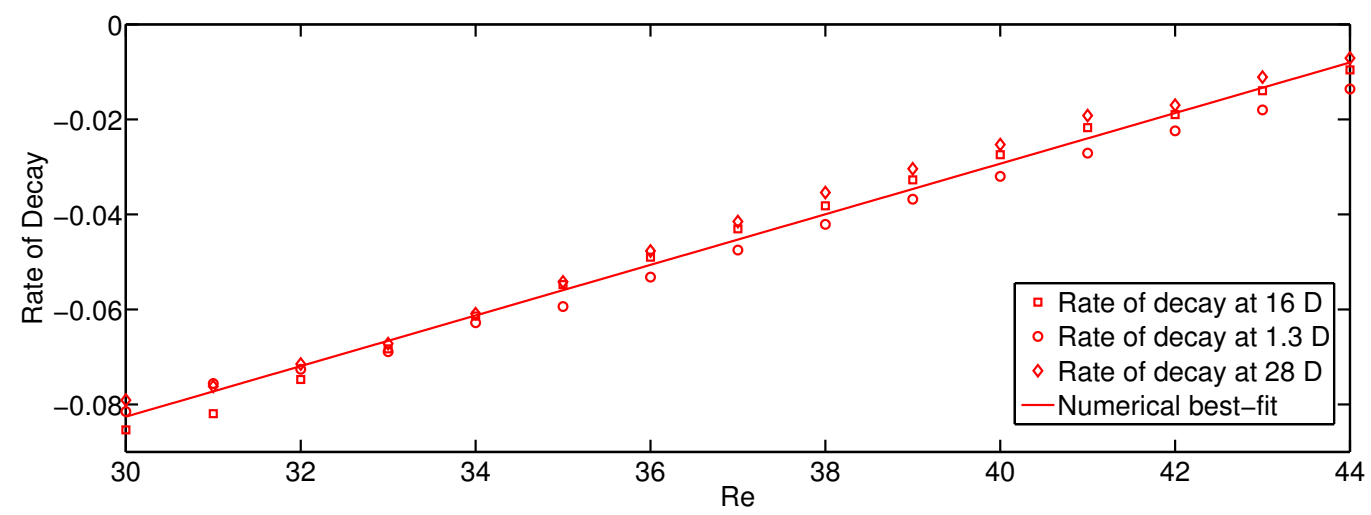

Figure 5.22. Rate of decay at the closed near-wake for $\mathrm{Re}=30-44$.

is called the time constant which gives us an idea how long it will take for the wake oscillations to disappear. Ideally, as $T_{t} \rightarrow \infty$, the enveloping function $\exp \left(-T_{t} / \tau\right) \rightarrow 0$.

Figure 5.22 shows the rate of decay of the wake oscillation at all the probe locations over the range of $30<R e<44$. It presents a linear functional relationship of $R e$ and the rate of decay, giving a best-fit line $R a D e=0.0053262 \times R e-0.24237$. Note that $R a D e$ are negative values. As Re increases, the magnitude of $R a D e$ decreases, which means the decaying of the wake oscillations slow down.

Thus, we argue that:

(i) The frequency of wake oscillation is determined by the condition of the flow, not the frequency of the perturbation (even some people get confused about this phenomenon and attribute these oscillations to the action of the perturbation itself).

(ii) When actually the energy oscillated with this oscillation seems to come from the flow itself. As if we were talking about kicking an active oscillator.

(iii) Both the characteristic frequency and the rate of decay of this oscillator seem to be directly related with the condition of the flow, especially, the Re and the topological distribution of the vortex structures in the wake.

\subsubsection{Theoretical Low-dimensional Model}

Building a Low-Dimensional Model (LDM) for various fluid systems has been of much interest. A LDM is is used to capture the essential features of the behavior vortex shedding 
and resolve multiple relevant degrees of freedom using a relatively simple model. This becomes an important technique to investigate in fluid dynamics, for both laminar and turbulent flows in nature and technology. The modeling of an LDM frequently starts from the experimental observations concerning the comparison between complex fluid motions and apparently simple Navier-Stokes equations. An LDM is derived with a fewer number of degrees of freedom from a system of non-linear fluid equations. The output of an LDM may not always have direct relationship to the experimental results. However, it provides a good chance to capture the basic physical mechanism qualitatively. Further, LDMs can usually explain the core dynamics to a certain extent, which is believed to be the main feature of the fluid motion [110].

The use of a proper orthogonal or Karhumen- Loéve decomposition (POD) was first introduced by Lumley [111]. This technique has been adopted in both experimental and numerical data to capture the kinetic energy of the system often better than any other method [112]. There is additional benefit in the models ability to order the modes by decreasing average energy content. With the POD technique, a LDM can be derived by performing a Galerkin projection of the Navier-Stokes equations onto a particular basis of spatial eigenfunctions. A group of ODEs are yielded to describe the behavior of the fluid system in time of the expansion coefficients [113].

The POD technique has been used to investigate many different fluid systems. Aubry et al. [114] reported its use to construct a LDM of a specific turbulent flow to study the coherent structures at turbulent boundary layer. Later, many researchers such as Sanghi and Aubry [115], Podvin and Lumley [116], Berkooz et al. [117], Sirovich [118], and Podvin [119], Sirovich and Zhou [120], Zhou and Sirovich [121], presented work on near-wall turbulence and channel flows [112]. Moehlis et al. [122] started a study of LDM of plane Couette flow and Smith et al. [123] continued this project for such flow in minimal flow unit based on the earlier work mentioned above. Ahuja et al. [124] studied the case of fluid dynamics of the vortices at leading edges of an airfoil when pitched up rapidly. In this study, an airfoil at various angles of attack was numerically modeled in a two-dimensional incompressible flow. The model developed is an approximation of balanced truncation that is applicable to large systems. Venturi et al. [125] extended POD to noisy (noncoherent) flows. In this study a new compact expansion of a random flow field into stochastic spatial modes was applied to unsteady laminar flow around a circular cylinder. Bailon-Cuba and Schumacher [126] developed a LDM for turbulent Rayleigh-Bénard convection in a Cartesian cell with square domain. This was done by the projection of the Boussinesq equations onto a finite set of empirical eigen-functions. These functions were obtained from a POD combing the velocity and temperature fields.

Another approach to construct a LMD for the dynamics of transitional flows is called "impressionistic models" Rempfer [113]. These models are in the form of sets of ODEs, the solutions from which approximately capture certain aspects of the flow dynamics. Baggett 


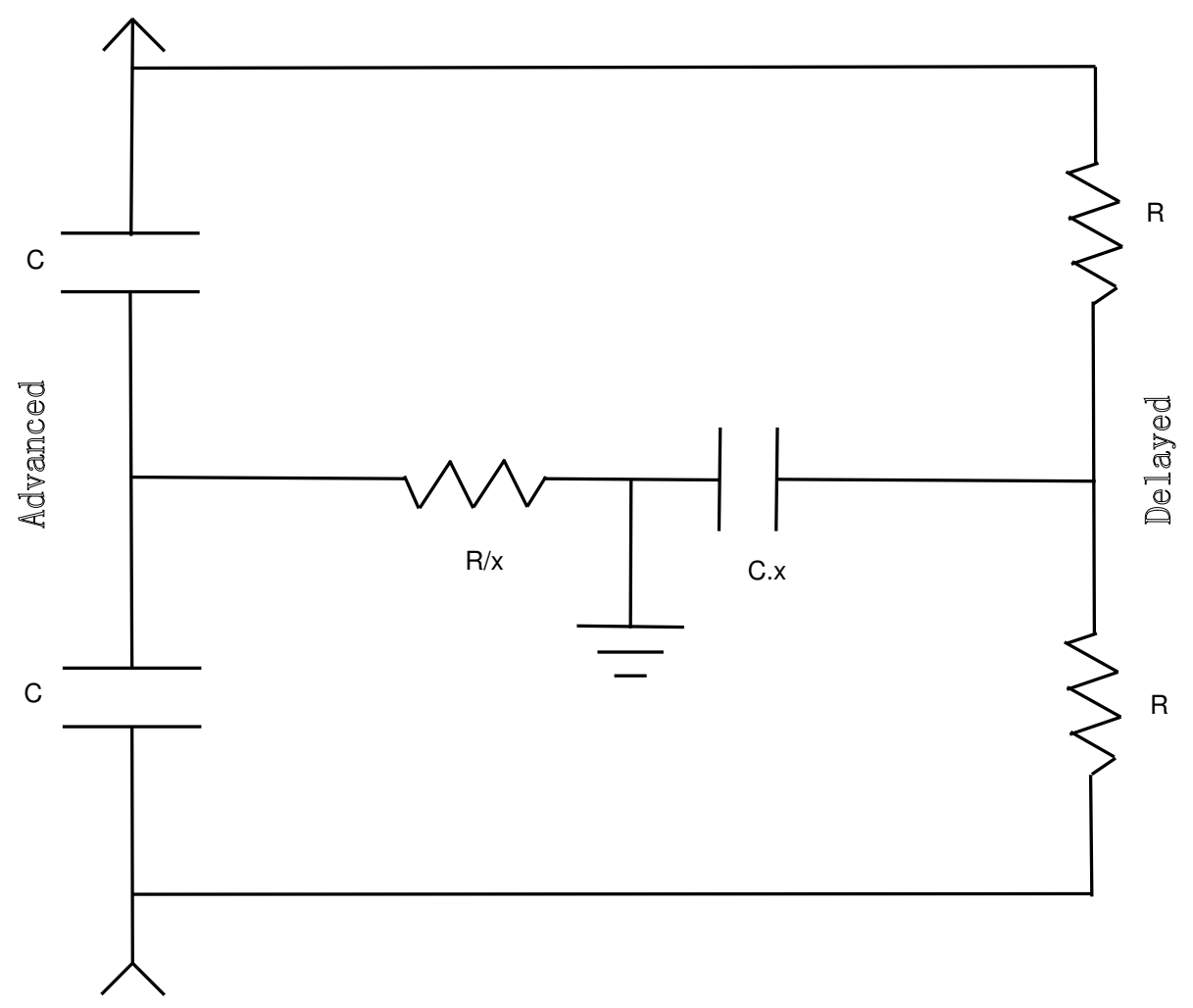

Figure 5.23. Twin-T filter circuit.

and Trefethen [127] showed some examples of such LDM representing the transition in linearly stable flows.

One hypothesis is that we could find an equivalent oscillator with a theoretical formula that could match this behavior, and with a circuital layout that matches the topological structure of the vorticity distribution of the wake in such a way that the exchange of the vorticity within the different elements explains the oscillations. The Low Dimensional Model proposed in this work is not based on the any method aforementioned, but borrows the basic idea to propose a model based on an analog circuit that is able to interpret the concerning flow by reducing the number of degrees of freedom. It follows the idea of the non-linear oscillator and resembles the dynamics mechanism of the closed near-wake with a common configured sine wave oscillator, the Twin-T oscillator as shown in figure 5.23. This kind of oscillator uses two "T" $R C$ circuits operated in parallel. Here, $R$ represents resistors and $C$ is for capacitors. The R-C-R "T" circuit acts as a low-pass filter while the C-R-C "T" circuit operates as a high-pass filter. When bridged together, these circuits are tuned at the desired oscillating frequency. In the C-R-C branch of the Twin-T oscillator, the signal is advanced, and that in the $\mathrm{R}-\mathrm{C}-\mathrm{R}$ branch is delayed. When $\mathrm{x}=2$, they may cancel one another under frequency of $f=\frac{1}{2 \pi R C}$ [128]. 
Figure 5.24 shows the two-dimensional sketch of the fluid dynamics model at the closed near-wake behind a cylinder at low Re. Two symmetric vortex "bubbles" are shown in different colors for the negative and positive vorticity. It is assumed this fluid system is in a stable state.

In Figure 5.24, wiring diagram symbols are used to describe this fluid system and the same parameters are mapped in the electrical circuit shown in Figure 5.25, $S_{1}$ and $S_{2}$ represent the power source, locating at the each side of the cylinder where the flow separation starts. $C_{1}$ through $C_{4}$ are for capacitors used. $R_{1}$ through $R_{4}$ are for the resistance. In this analogy, it is proposed that current is an anolog for vorticity, electric capacitance is an anolog for ciculation and elecric potential (voltage) is an anolog for gradient of vorticity. When the flow separation is initiated, the resulting vorticity forms two vortex cores of opposite signs behind the cylinder and the accumulation process of the vorticity is similar to how a capacitor collects electric current, represented by $C_{1}$ and $C_{2}$ as shown in the Figure 5.24, The flow resistance during this process is represented by $R_{1}$ and $R_{2}$ respectively in Figure 5.24. At the wake axis where the vorticity of different signs meet, the dotted line in Figure 5.24, the disturbed flow experiences a cancellation of vorticity, in other words, flow resistance denoted as $R_{3}$; at the same time, $C_{3}$ is assumed to maintain the stability of this fluid system. As for $R_{4}$ and $C_{4}$, these parameters represent the flow resistance and vorticity beyond the confluence point in the closed near-wake, which will become more important as Re increases. The wake axis is represented by the "Ground" in the electrical circuit. The output of analog circuit, a sine wave, resembles the wake oscillation in the actual fluid system. 


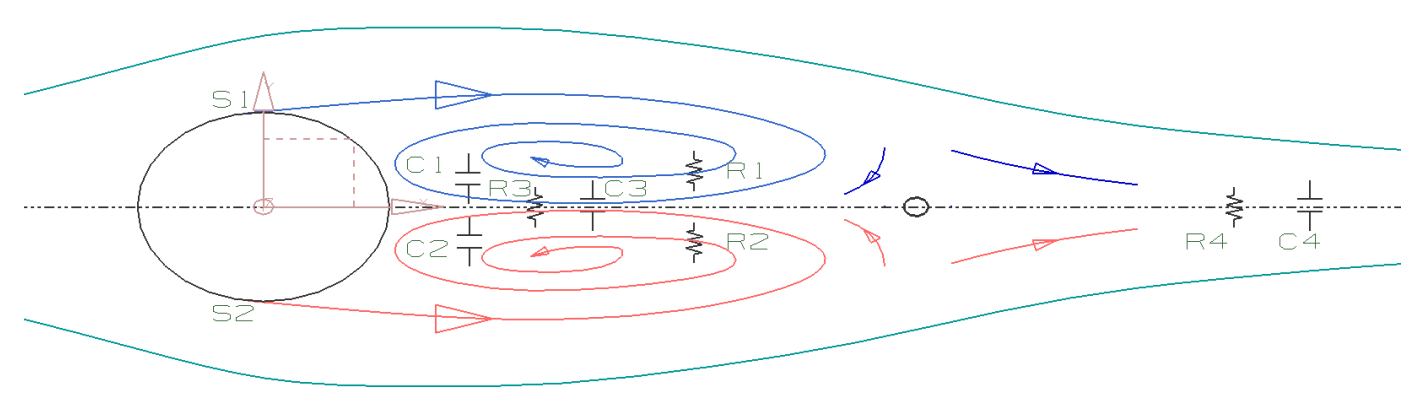

Figure 5.24. Vortex Oscillator model.

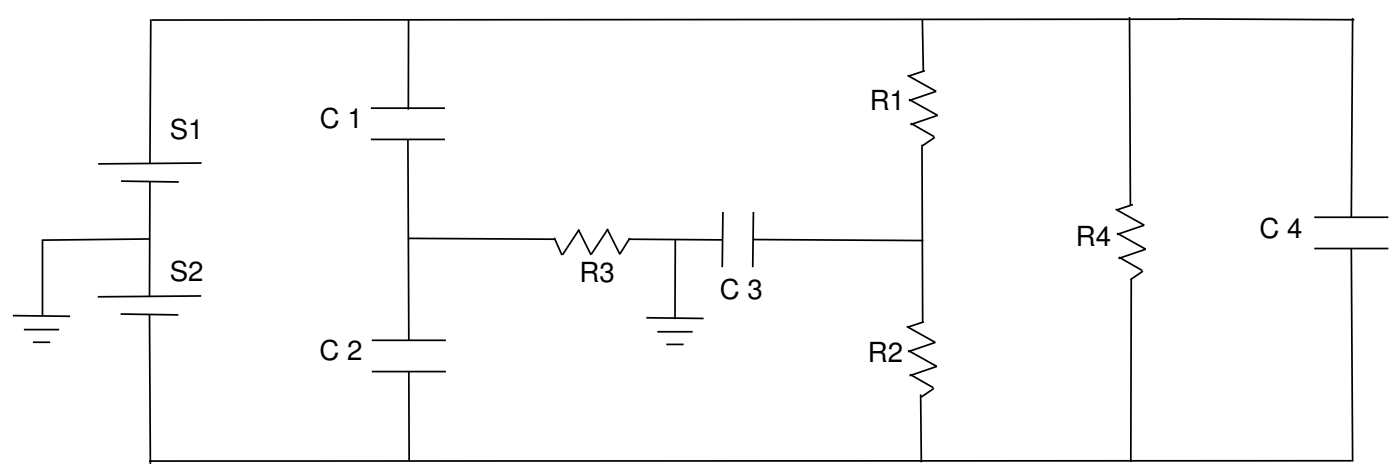

Figure 5.25. Diagram of the vortex oscillator model.

\subsection{Re-St relationship}

It has been challenging question to understand the Strouhal - Reynolds number relationship over almost a century. The extensive measurements during the past five decades have advanced the study of the Strouhal - Reynolds relationship in the case of flow around bluff-bodies in laminar regime. It was Roshko [55] who first introduced the parameter $R o=S t \times R e=\frac{f \times D^{2}}{\nu}$ versus $\mathrm{Re}$, where $\mathrm{f}$ is the shedding frequency, $\mathrm{D}$ is the characteristic length of the body, and the Ro is the Roshko number. According to Roshko's work, a linear least-squares fit for the $R o-R e$ plot was found and the resulting functional relationship of St-Re is given in terms of $1 / R e$ as follows:

$$
S t=A-\frac{B}{R e}
$$

There have been a large number of fitting curves of this St-Re relationship published after Roshko's work. However, there was little agreement among these studies. A controversy 
about the nature and locations of several discontinuities observed in the data was largely resolved with Williamson's measurement [129]. It was found that, if parallel shedding is obtained by manipulating the boundary conditions at the end of the cylinder, a continuous St-Re curve throughout the laminar range $(49<R e<178)$ can be made. Now, this specific St-Re curve is considered to be universal and it represents measurements for the case of purely two-dimensional vortex shedding [7].

Later, Fey et al. [130] reported a functional relationship of St versus $1 / \sqrt{R e}$ which is visually straight for the cases of laminar flow. At about the same time, Williamson and Brown [56] showed a new St-Re relationship of the same parameters for the cylinder wake, which showed one order of magnitude less error than the traditional St versus $1 / R e$ curves. Lord Rayleigh suggested that a Taylor's expansion of $1 / R e$ can be used to express St [131]. Following this idea, a series expansion in terms of $1 / \sqrt{R e}$ were proposed by [56] as follows:

$$
S t=(A+B / \sqrt{R e}+C / R e+\ldots)
$$

The parallel-shedding St-Re data in the laminar flow regime obtained by Williamson [129] was used in the least-squares fitting with the use of truncation in Eq 5.5 upto the first two terms. A comparison was made with the traditional two-term fitting in functional relationship of $S t-1 / R e$. Compared to the traditional $1 / R e-f i t$, a more favourable agreement to the parallel-shedding St-Re data was found with the $\sqrt{R e}-$ formula [129].

To interpret the St-Re relationship, Williamson and Brown [56] proposed a physical connection of two length scales. First, the wake width $\left(L^{*}\right)$, and second, the vorticity-thickness of the separated shear layers $\left(\delta_{w}\right)$ were used to interpret the $1 / \sqrt{R e}-f i t$. The constant term $A$ in the $\sqrt{R e}$-formula was interpreted with the physical shape/size of bluff bodies and the subsequent terms of $1 / \sqrt{R e}$ are interpreted with the thickness of the separated shear layers.

Multiple theoretical models have been developed for the StRe number relationship. A phenomenological model for vortex-street formation initiated by a bluff body was developed by Ahlborn et al. [132], where the mass, momentum and energy balances were analyzed. This method defined the correlation between the drag coefficients, Re and the Strouhal number. A two-parameter formula $S t=1 /(A+B / R e)$ was developed by Roushan and $\mathrm{Wu}$ [133]. The observations of the vortex street structure in this work was done with the experiments in flowing soap films.

In the preceding work by Ponta and Aref [134], it was proposed that the empirical St-Re is natural and can be derived from an elucidation of the mechanism of vortex formation. This 
observation can be done numerically with the estimation of the vorticity transport terms. To achieve this, the diameter of the bluff body, D, was adopted as the characteristic length scale which would then govern the concerning physical processes. This method resulted in a model that had a good correlation to the traditional $1 / R e$-fit. Going further Ponta [135] replaced D as the measure of the characteristic length with the shear layers thickness. This change in how the vorticity diffusion is governed during the vortex-formation process resulted in a model that fits the $1 / \sqrt{R e}$-fit.

However, the Strouhal-Reynolds number relationship at low Re, over the range of $R e<49$, have not yet been widely studied. Camichel and Teissié-Solier [98] measured the frequency of the artificially excited near-wake for $R e<R e_{o s c}$. Their result shows the relationship of a non-dimensional Strouhal number, $S t=f * \frac{D}{U}$ verse $\operatorname{Re}$ for $R e>R e_{o s c}$. The experimental data was obtained from the self-excited near-wake oscillations. The disturbed cylinder produced a temporary periodic near-wake down to $R e=20$. Camichel and Teissié-Solier [98] found an even more effective way to destabilize the near-wake down to $R e=10$. The opening of a valve at the side of the water tunnel produced a transverse disturbance across the test section and triggered the near-wake instability. He reported the forced and natural $S t-R e$ curve, which showed that the result obtained by artificial excitation of the near-wake merged smoothly into the curve obtained by the self-excited near-wake.

Following Roshko's idea, we study St-Re Relationship numerically over the range of $16<$ $R e<47$ and make a comparison to Camichel's experiments. In Camichel's experiment, he reported the St-Re relationship down to $R e=10$ approximately. The comparison made is for $16<R e<47$ because the St values at $10<R e<16$ are not consistent with those at the rest Re in this range. This could be due to his experiment set-ups. Figure 5.26 shows the comparison of $S t \times R e$ verse Re with Camichel's experiment. As shown in the figure, the numerical result fits in a linear line, which is visually the offset of Camichel's data with the best fit lines of $S t R e=0.13704 \times R e-0.87349$ for the numerical data and $S t R e=$ $0.134 \times R e-1.1747$ for Camichel's. These two best-fit lines are almost parallel, which indicates the frequencies of the wake oscillations at low Re follow a consistent tendency with respect to the change of Re. The tendency observed in our numerical experiments is consistent and repetitive for all the probing points and over a range of different mesh designs (i.e., no grid dependence was observed). The offset shown in this figure could be attributed to Camichel's experimental set-up. It is known that blockage effects may offset the shedding frequency curve. Unfortunately, the description of the measurement techniques at the time when Camichel's study was performed is limited, and there is a lack of more recent data in the available literature. Further analysis of this topic would require acquisition of new data by modern techniques. 


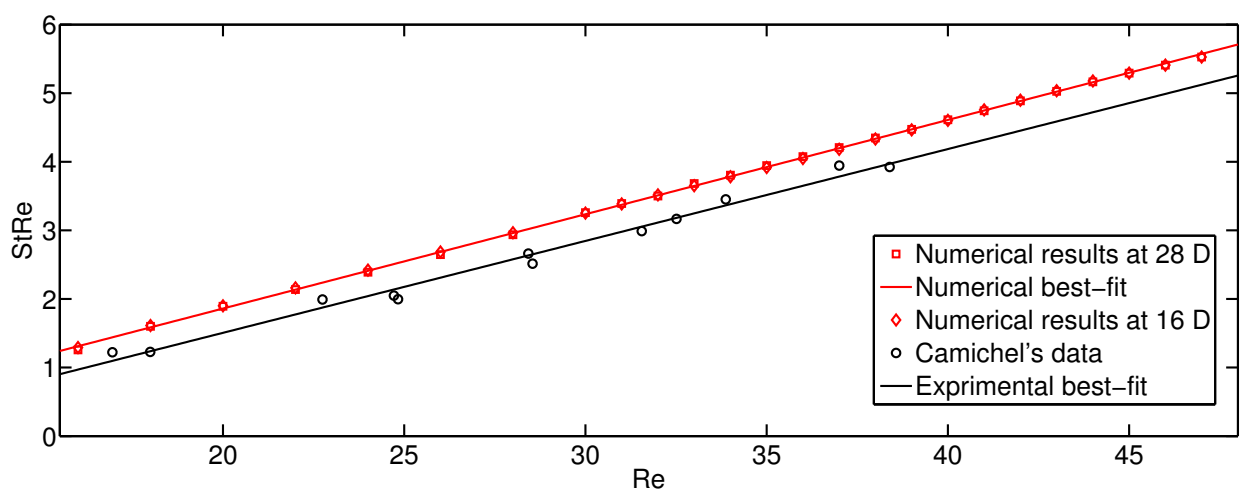

Figure 5.26. Comparison of StRe vs Re with Camichel's experiment at $R e=16-47$. 


\section{Conclusions}

In this dissertation we studied the underlying physics of vortex-shedding and wake dynamics in long aspect-ratio aerodynamics in incompressible viscous flow through the use of the KLE method. We carried out a long series of numerical experiments in the cases of flow around the cylinder at low Reynolds numbers. As it was mentioned above, these studies of flows at low Reynolds numbers provide an insight in the fluid physics which plays a critical role on phenomena connected to stalled wind-turbine rotors. Many of the conclusions about the qualitative nature of the physical mechanisms characterizing vortex formation, shedding and further interaction analyzed here at low Re could be extended to other Re regimes and help to understand the separation of the boundary layers in airfoils and other aerodynamic surfaces. A better understanding of the complex multi-physics problems involving streamlined and bluff-body conditions will also serve as a valuable guide for the future design of blade aerodynamics and the placement of wind turbines and hydrokinetic turbines, increasing the efficiency in the use of expensive workforce, supplies, and infrastructure.

After the introductory section describing the main fields of application of wind power and hydrokinetic turbines, we described the main features and theoretical background of the numerical method used here. Then, we presented the analysis of the numerical experimentation results for the oscillatory regime right before the onset of vortex shedding for circular cylinders. We verified the wake length of the closed near-wake behind the cylinder and analysed the decay of the wake at the wake formation region, and then studied the St-Re relationship at the Reynolds numbers before the wake sheds compared to the experimental data. We found a theoretical model that describes the time evolution of the amplitude of fluctuations in the vorticity field on the twin vortex wake, which accurately matches the numerical results in terms of the frequency of the oscillation and rate of decay. We also proposed a model based on an analog circuit that is able to interpret the concerning flow by reducing the number of degrees of freedom. It follows the idea of the non-linear oscillator and resembles the dynamics mechanism of the closed near-wake with a common configured sine wave oscillator. This low-dimensional circuital model may also help to understand the underlying physical mechanisms, related to vorticity transport, that give origin to those oscillations. 
In Chapter 5, we presented the analysis of the numerical results for the oscillatory regime right before the onset of vortex shedding for circular cylinders. The decay of wake oscillation behind circular cylinder in a 2-D steady flow for low Re was successfully reported. In the numerical experiments performed, we found that the wake oscillations fall into a sinusoidal pattern "bounded" by two exponential decaying lines defined by the peaks values of the vorticity. The oscillations at three locations for a specified Re show consistency in physical characteristics. The oscillations are damping out as time goes by with different decay and they oscillate under similar frequency. As Re increases, the viscous effect becomes weaker, and the oscillation tends to damping slower and survives further downstream. This fluid phenomenon is also successfully studied quantitatively. We found a theoretical model that describes the time evolution of the amplitude of fluctuations in the vorticity field on the twin vortex wake, which accurately matches the numerical results in terms of the frequency of the oscillation and rate of decay as shown in Eq 5.3. The rate of decay of the wake oscillation at all the probe locations over the range of $30<R e<44$ is also presented. It is a linear functional relationship of Re and the rate of decay, giving a best-fit line $R a D e=0.0053262 \times R e-0.24237$. Note that $R a D e$ are negative values. As Re increases, the magnitude of $R a D e$ decreases, which means the decaying of the wake oscillations slow down.

We verified the wake length of the closed near-wake behind the cylinder and analysed the decay of the wake at the wake formation region, and then studied the St-Re relationship at the Reynolds numbers before the wake sheds compared to the experimental data. The StRe Relationship over the range of $16<R e<47$ is studied numerically and a comparison to Camichel's experiments is made. As shown in Figure 5.26, the numerical result fits in a linear line, which is visually the offset of Camichel's data with the best fit lines of $S t R e=0.13704 \times R e-0.87349$ for the numerical data and $S t R e=0.134 \times R e-1.1747$ for Camichel's. These two best-fit lines are almost parallel, which indicates the frequencies of the wake oscillations at low Re follow a consistent tendency with respect to the change of Re. The tendency observed in our numerical experiments is consistent and repetitive for all the probing points and over a range of different mesh designs (i.e., no grid dependence was observed).

We also proposed a model based on an analog circuit that is able to interpret the concerning flow by reducing the number of degrees of freedom. It follows the idea of the non-linear oscillator and resembles the dynamics mechanism of the closed near-wake with a common configured sine wave oscillator. This low-dimensional circuital model may also help to understand the underlying physical mechanisms, related to vorticity transport, that give origin to those oscillations. Figure 5.24 shows the two-dimensional sketch of the fluid dynamics model at the closed near-wake behind a cylinder at low Re. Two symmetric vortex "bubbles" are shown in different colors for the negative and positive vorticity. It is assumed this fluid system is in a stable state. In Figure 5.24, wiring diagram symbols are used to describe this fluid system and the same parameters are mapped in the electrical 
circuit shown in Figure 5.25, In this analogy, it is proposed that current is an anolog for vorticity, electric capacitance is an anolog for circulation and electric potential (voltage) is an anolog for gradient of vorticity. The output of analog circuit, a sine wave, resembles the wake oscillation in the actual fluid system.

As an outlook for future work, a deeper study of the low dimensional model based on the electrical analogy would be interesting. With LDM, it is possible to characterize both the rate of decay and the frequency of the wake oscillations (Re-St relationship), and the mechanisms of the underlying physics. 


\section{REFERENCES}

(1) DOE, Wind power today Federal wind program highlights, Technical Report, U.S Department of Energy, 2005.

(2) S. Butterfield, W. Musial, J. Jonkman, P.Sclavounos, Engineering Challenges for Floating Offshore Wind Turbines, Technical Report, National Renewable Energy Laboratory, 2005.

(3) ICT-URL, About aeolus, 2007. (accessed Feb 2012).

(4) R. Bedard, Priortized Research, Development,Deployment and Demonstration(RDD\&D) Needs: Marine and other Hydrokinetic Rewable Energy, Technical Report, Electric Power Research Institute, 2008.

(5) M. G. U. Lundin, M. Leijon, Ocean energy, 2010. (accessed Jan 2010).

(6) F. L. Ponta, The kinematic Laplacian equation method, J. Comput. Phys. 207 (2005) $405-426$.

(7) C. H. K. Williamson, Oblique and parallel mode of vortex shedding in the wake of a circular cylinder at low Reynolds numbers, J. Fluid Mech. 206 (1989) 579-627.

(8) F. Ponta, Vortex decay in the kármán eddy street, Physics of Fluids 22 (2010) 093601.

(9) C. H. K. Williamson, A. Roshko, Vortex formation in the wake of an oscillating cylinder, J. Fluids Struct. 2 (1988) 355-381.

(10) F. L. Ponta, H. Aref, Numerical experiments on vortex shedding from an oscillating cylinder, J. Fluids Struct. 22 (2006) 327-344.

(11) F. L. Ponta, H. Aref, Vortex synchronization regions in shedding from an oscillating cylinder, Phys. Fluids 17 (2005) 011703.

(12) S. Taneda, Experimental investigation of the wakes behind cylinders and plates at low reynolds numbers, Journal of Physical Society of Japan 11 (1956) 302-7. 
(13) C. H. K. Williamson, R. Govardhan, Vortex-induced vibrations, Annu. Rev. Fluid Mech. 36 (2004) 413-455.

(14) H. Fasel, Investigation of the stability of boundary layers by a finite difference model of the Navier-Stokes equations, J. Fluid Mech. 78 (1976) 355-383.

(15) H. J. H. Clercx, A spectral solver for the navier-stokes equations in the velocityvorticity formulation for flows with two nonperiodic directions, J. Comput. Phys. 137 (1997) 186-211.

(16) WWEA, World Wind Energy Report 2009, Report, World Wind Energy Association, 2010. Www . wwindea. org.

(17) N. Hodge, A snapshot of the global wind industry, 2008. (accessed Feb 2010).

(18) NREL, Wind Power Today, Report DOE/GO-102005-2115, U.S. Department of Energy, 2005.

(19) E. de Vries, Thinking bigger: Are there limits to turbine size?, Renewable Energy World 8 (2005).

(20) H. Hillewaert, Windmills D1-D4 (Thornton Bank), http://en.wikipedia •org/ wiki/File:Windmills_D1-D4_(Thornton_Bank).jpg, 2009. See Appendix for documentation of permission to republish this material.

(21) A. D. Hansen, F. Iov, F. Blaabjerg, L. H. Hansen, Review of contemporary wind turbine concepts and their market penetration, Wind Engineering 28 (2004) 247263.

(22) L. H. Hansen, L. Helle, F. Blaabjerg, E. Ritchie, S. Munk-Nielsen, H. Bindner, P. Sørensen, B. Bak-Jensen, Conceptual survey of generators and power electronics for wind turbines (2001).

(23) R. Oman, K. M. Foreman, Advantages of the diffuser-augmented wind turbine, NASA. Lewis Res. Center Wind Energy Conversion Systems (1973) p 103-106.

(24) L. Chen, F. L. Ponta, L. I. Lago, Perspectives on innovative concepts in wind-power, Energy for Sustainable Development 15 (2011) 398-410.

(25) ScienceDaily-URL, Deep-sea oil rigs inspire designs for giant wind turbines, 2006. (accessed Feb 2010).

(26) W. D. Musial, C. P. Butterfield, A. Boone, Feasibility of floating platform systems for wind turbines, 23rd ASME Wind Energy Symposium Proceedings (2004). NREL/CP-500-34874. 
(27) J. N. Newman, Marine hydrodynamics, The MIT Press (1977). ISBN 0-262-140268.

(28) A. R. Henderson, J. Vugts, Prospects for floating offshore wind energy (2001).

(29) J. F. Manwell, J. G. McGowan, A. L. Rogers, Wind energy explained: Theory, design and application, Wiley, 2002.

(30) T. Johansson, L. Burnham, Renewable energy: sources for fuels and electricity, Island Press, 1993.

(31) L. I. Lago, F. L. Ponta, L. Chen, Advances and trends in hydrokinetic turbine systems, Energy for Sustainable Development 14 (2010) 287-296.

(32) A. S. Bahaj, L. E. Myers, Fundamentals applicable to the utilisation of marine current turbines for energy production, Renewable Energy 28 (2003) 2205-2211.

(33) G. Buigues, I. Zamora, A. Mazon, V. Valverde, E. Perez, Sea energy conversion: problems and possibilities (2006).

(34) R. Bedard, overview of technology classes and key terminology, in: S. S. Schwartz (Ed.), Proceedings of the Hydrokinetic and Wave Energy Technologies Technical and EnvironmentalIssues Workshop.Washington, DC., RESOLVE, Inc., Washington, D.C., USA, 2005, pp. 5-8.

(35) M. Khan, G. Bhuyan, M. Iqbal, J. Quaicoe, Hydrokinetic energy conversion systems and assessment of horizontal and vertical axis turbines for river and tidal applications: A technology status review, Applied Energy 86 (2009) 1823-1835.

(36) F. L. Ponta, Kinematic laplacian equation method: A velocity-vorticity formulation for the navier-stokes equations, Journal of Applied Mechanics 73 (2006) 1031-1038.

(37) M. M. Zdravkovich, Flow around circular cylinders, volume 1, Oxford University Press, Oxford, UK, 1997.

(38) M. Van Dyke, An album of fluid motion, Parabolic Press, Stanford, California, USA, 1982.

(39) F. L. Ponta, Analyzing the vortex dynamics in bluff-body wakes by Helmholtz decomposition of the velocity field, Fluid Dynamics Research 38 (2006) 431-451.

(40) G. K. Batchelor, An introduction to fluid dynamics, Cambridge University Press, Cambridge, UK, 2000.

(41) T. von Kármán, H. Rubach, On the mechanism of resistance in fluids (in German), Physikalische Zeitschrift 13 (1912) 49-59. 
(42) T. von Kármán, Collected works of Theodore von Kármán, volume 1, Butterworths, 1956.

(43) M. Bénard, On the incorrectness, for real fluids, of Kármán's theory of stability of alternate vortices (in French), Comp. Rend. Acad. Sci. 182 (1926) 1523-1525.

(44) S. G. Hooker, On the action of viscosity in increasing the spacing ration of a vortex street, Proc. Roy. Soc. A154 (1936) 67-89.

(45) H. Lamb, Hydrodynamics 6th ed., Cambridge University Press, Cambridge, UK, 1932.

(46) A. Timme, On the velocity distribution in eddies (in German), Ingenieur Archiv 25 (1957) 205-225.

(47) J. W. Schaefer, S. Eskinazi, An analysis of the vortex street generated in viscous fluid, J. Fluid Mech. 6 (1959) 241-260.

(48) R. B. Green, J. H. Gerrard, An optimal interferometric study of the wake of a bluff body, J. Fluid Mech. 226 (1991) 219-242.

(49) J. B. Flór, G. J. F. van Heijst, An experimental study of dipolar structures in a stratified fluid, J. Fluid Mech. 279 (1994) 101-133.

(50) M. A. Stremler, Relative equilibria of vortex arrays, 2003. Preprint.

(51) O. M. Griffin, S. E. Ramberg, The vortex street wakes of vibrating cylinders, J. Fluid Mech. 66 (1974) 553-576.

(52) A. Khalak, C. H. K. Williamson, Motion forces and mode transitions in vortexinduced vibration at low mass-damping, J. Fluids Struct. 13 (1999) 813-851.

(53) R. Govardhan, C. H. K. Williamson, Modes of vortex formation and frequency response of a freely vibrating cylinder, J. Fluid Mech. 420 (2000) 85-130.

(54) J. R. Meneghini, P. W. Bearman, Numerical simulation of high amplitude oscillatory flow about a circular cylinder, J. Fluids Struct. 9 (1995) 435-455.

(55) A. Roshko, On the development of turbulent wakes from vortex streets, TR 1191, NACA, 1954.

(56) C. H. K. Williamson, G. L. Brown, A series in $1 / \sqrt{R e}$ to represent the StrouhalReynolds number relationship of the cylinder wake, J. Fluids Struct. 12 (1998) 1073-1085.

(57) H. Aref, E. Siggia, Evolution and breakdown of a vortex street in two dimensions, J. Fluid Mech. 109 (1981) 435-463. 
(58) J. M. Cimbala, H. M. Nagib, A. Roshko, Large structure in the far wakes of twodimensional bluff bodies, J. Fluid Mech. 190 (1988) 265-298.

(59) O. Inoue, T. Yamazaki, Secondary vortex streets in Two-dimensional cylinder wakes, Fluid Dyn. Res. 25 (1999) 1-18.

(60) T. Matsui, M. Okude, Formation of the secondary vortex street in the wake of a circular cylinder, in: IUTAM Symposium on Structures of Compressible Turbulent Shear Flows, Marseille, Springer-Verlag, 1983, pp. 156-164.

(61) E. Meiburg, On the role of subharmonic perturbations in the far wake, J. Fluid Mech. 177 (1987) 83-107.

(62) S. Taneda, Downstream development of the wakes behind cylinders, J. Phys. Soc. of Japan 14 (1959) 843-848.

(63) C. H. K. Williamson, A. Prasad, A new mechanism for oblique wave resonance in the natural far wake, J. Fluid Mech. 256 (1993) 269-313.

(64) D. Rempfer, On boundary conditions for incompressible navier-stokes problems, Applied Mechanics Reviews 59 (2006) 107-125.

(65) R. Glowinski, Finite element methods for navier-stokes equations, Annual Review of Fluid Mechanics 24 (1992) 107-125.

(66) L. Quartapelle, Numerical solution of the incompressible Navier-Stokes equations, Birkäuser, Basel, Switzerland, 1993.

(67) L. Quartapelle, M. Napolitano, Integral conditions for the pressure in the computation of incompressible viscous flows, Journal of Computational Physics 62 (1986) 340-348.

(68) C. Fletcher, Computational Techniques for Fluid Dynamics Vol.2, Springer, second edition, 1988.

(69) D. Kwak, C. Kiris, C. S. Kim, Computational challenges of viscous incompressible flows, Computers and Fluids 34 (2005) 283-299.

(70) S. Kempka, J. Strickland, M. Glass, J. Peery, P. M. Ingber, Velocity boundary conditions for vorticity formulations of the incompressible navier-stokes equations, in: Forum on the Application of Vortex Methods to Engineering Problems, The U.S. Dept. of Energy, 1995.

(71) T. B. Gatski, Review of incompressible fluid flow computations using the vorticityvelocity formulation, Applied Numerical Mathematics 7 (1991) 227-239. 
(72) C. Davies, P. W. Carpenter, A novel velocity-vorticity formulation of the navier-stokes equations with applications to boundary layer disturbance evolution, Journal of Computational Physics 172 (2001) 119-165.

(73) J. Z. W. X. H. Wu, J. M. Wu, Effective vorticity-velocity formulations for 3d incompressible viscous flows, Journal of Computaional Physics 122 (1995) 68-82.

(74) G. E. Karniadakis, S. Sherwin, Spectral/hp Element Methods for CFD, Oxford University Press, second edition, 2005.

(75) F. L. Ponta, P. M. Jacovkis, A vortex model for Darrieus turbine using finite element techniques, Renewable Energy 24 (2001) 1-18.

(76) F. L. Ponta, P. M. Jacovkis, Constant-curl Laplacian equation: a new approach for the analysis of flows around bodies, Computers and Fluids 32 (2003) 975-994.

(77) C. R. Anderson, Observations on vorticity creation boundary conditions, in: R. E. Caflisch (Ed.), Mathematical Aspects of Vortex Dynamics, SIAM, 1988, pp. 144159.

(78) A. J. Chorin, Numerical study of slightly viscous flow, J. Fluid Mech. 57 (1973) 785-796.

(79) A. J. Chorin, Vortex sheet approximation of boundary layers, J. Comput. Phys. 27 (1978) 428-442.

(80) J. P. Boyd, Chebyshev and Fourier spectral methods, Dover, Mineola, New York, USA, 2000.

(81) S. J. Sherwin, G. E. Karniadakis, Tetrahedral hp finite elements: Algorithms and flow simulations, J. Comput. Phys. 124 (1996) 14-45.

(82) M. C. Thompson, K. Hourigan, J. Sheridan, Three-dimensional instabilities in the wake of a circular cylinder, Exp. Therm. and Fluid Science 12 (1996) 190-196.

(83) G. E. Karniadakis, M. Israeli, S. A. Orszag, High-order splitting methods for the incompressible navier-stokes equations, J. Comput. Phys. 97 (1991) 414-443.

(84) M. A. Sprague, T. L. Geers, Legendre spectral finite elements for structural dynamics analysis, Communications in Numerical Methods in Engineering 00 (2007) 1-13.

(85) A. T. Patera, A spectral element method for fluid dynamics: laminar flow in a channel expansion, J. Comput. Phys. 54 (1984) 468-488.

(86) G. E. Karniadakis, E. T. Bullister, A. T. Patera, A spectral element method for solution of two- and three-dimensional time-dependent incompressible navier-stokes equations, in: Finite Element Methods for Nonlinear Problems, Springer-Verlag, New York/Berlin, 1985, p. 803. 
(87) R. D. Henderson, G. E. Karniadakis, Unstructured spectral element methods for simulation of turbulent flows, J. Comput. Phys. 122 (1995) 191-217.

(88) K. Hourigan, M. C. Thompson, B. T. Tan, Self-sustained oscilations in flows around long blunt plates, J. Fluids Struct. 15 (2001) 387-398.

(89) F. X. Giraldo, The Lagrange-Galerkin spectral element method on unstructured quadrilateral grids, J. Fluid Mech. 147 (1998) 114-146.

(90) K. J. Bathe, Finite element procedures, Prentice Hall, Englewood Cliffs, New Jersey, USA, 1996.

(91) D. Pathria, G. E. Karniadakis, Spectral element method for elliptic problems in nonsmooth domains, J. Comput. Phys. 122 (1994) 83-95.

(92) G. F. C. A. M. P. Valli, A. L. G. A. Coutinho, Control strategies for timestep selection in finite element simulation of incompressible flows and coupled reactionÚconvectionÜdiffusion processes, International Journal for numerical methods in fluids 47 (2005) 201-231.

(93) L. F. Shampine, M. K. Gordon, Computer solution of ordinary differential equations: the initial value problem, W. H. Freeman, San Francisco, California, USA, 1975.

(94) A. U. George Hall, Modified order and stepsize strategies in adams codes, Journal of Computational and Applied Mathematics 111 (1999) 113-122.

(95) H. Nisi, A. W. Porter, On eddies in air, Philosophical Magazine 46 (1923) 754-70.

(96) M. Coutanceau, R. Bouard, Experimental determination of the main features of the viscous flow in the wake of a circular cylinder in uniform translation part $\mathrm{i}$; steady flow., Journal of Fluid Mechanics 79 (1977) 231-6.

(97) C. Camichel, C. Dupin, M. Teissié-Solier, On the application of similarity law to the periodic formation of alternate bénard-kármán eddies(in french), Comptes Rendus Academie Sciences 185 (1927) 1536-9.

(98) C. Camichel, M. Teissié-Solier, Influence of perturbations on the wake around immersed body in the laminar regime(in french), Comptes Rendus Academie Sciences 200 (1935) 704-7.

(99) S. Taneda, The stability of two-dimensional laminar wakes at low reynolds number, Journal of Physical Society of Japan 18 (1963) 288-96.

(100) J. H. Gerrard, The wakes of cylindrical bluff bodies at low reynolds number, Philosophical Transactions A288 (1978) 351-82. 
(101) C. H. K. Williamson, Vortex dynamics in the cylinder wake, Annu. Rev. Fluid Mech. 28 (1996) 477-539.

(102) L. Föppl, Vortex motion behind a circular cylinder (in german), Bayerische Akademie des Wissenschaften, Müchen (1913).

(103) H. Honji, Flow around a circular cylinder in a converging or diverging rectangular working section, Reports Research Institute for Applied Mechanics, Kyushu University 17 (1969) 249-251.

(104) A. Thom, The flow past circular cylinders at low speeds, Proceedings of Royal Society A141 (1933) 651-69.

(105) A. S. Grove, F. H. Shair, E. E. Petersen, A. Acrivos, An experimental investigation of the steady separated flow past a circular cylinder, Journal of Fluid Mechanics 19 (1964) 60-80.

(106) F. Homann, Influence of higher viscosity on flow around cylinder (in german), Forschung aus dem Gebiete des Ingenieurwesen 17 (1936) 1-10. Transl 1952. NACA TM 1334.

(107) L. S. G. Kovasznay, Hot-wire investigations of the wake behind cylinders at low reynolds numbers, Proceedings of Royal Society A198 (1949) 174-90.

(108) M. Nishioka, H. Sato, Mechanism of determination of the shedding frequency of vortices behind a cylinder at low reynolds numbers, Journal of Fluid Mechanics 89 (1978) 49-60.

(109) A. Thom, The strength and position of the eddies behind a circular cylinder, Aeronautical Research Council, Rep. \& Memo. 1373 (1930).

(110) A. Meseguer, F. Marques, J. Sanchez, Feigenbaum's universality in a lowdimensional fluid model, International Journal of Bifurcation and Chaos in Applied Sciences and Engineering 6 (1996) 1587-1594.

(111) J. Lumley, Stochastic tools in turbulence, Dover Publications, 2007.

(112) T. Smith, J. Moehlis, P. Holmes, Low-dimensional modelling of turbulence using the proper orthogonal decomposition: a tutorial, Nonlinear Dynamics 41 (2005) $275-307$.

(113) D. Rempfer, Low-dimensional modeling and numerical simulation of transition in simple shear flows, Annual review of fluid mechanics 35 (2003) 229-265.

(114) N. Aubry, P. Holmes, J. LUMLEY, E. Stone, The dynamics of coherent structures in the wall region of a turbulent boundary layer, Journal of Fluid Mechanics 192 (1988) 173355. 
(115) S. Sanghi, N. Aubry, Mode interaction models for near-wall turbulence, Journal of Fluid Mechanics 247 (1993) 455-488.

(116) B. Podvin, J. Lumley, A low-dimensional approach for the minimal flow unit, Journal of Fluid Mechanics 362 (1998) 121-155.

(117) G. Berkooz, P. Holmes, J. Lumley, Intermittent dynamics in simple models of the turbulent wall layer, Journal of Fluid Mechanics 230 (1991) 75-95.

(118) L. Sirovich, Turbulence and the dynamics of coherent structures. part i-iii, Quarterly of applied mathematics 45 (1987) 561-571.

(119) B. Podvin, On the adequacy of the ten-dimensional model for the wall layer, Physics of fluids 13 (2001) 210.

(120) L. Sirovich, X. Zhou, Dynamical model of wall-bounded turbulence, Physical review letters 72 (1994) 340-343.

(121) X. Zhou, L. Sirovich, Coherence and chaos in a model of turbulent boundary layer, Physics of Fluids A: Fluid Dynamics 4 (1992) 2855.

(122) J. Moehlis, T. Smith, P. Holmes, H. Faisst, Models for turbulent plane couette flow using the proper orthogonal decomposition, Physics of Fluids 14 (2002) 2493.

(123) T. Smith, J. Moehlis, P. Holmes, Low-dimensional models for turbulent plane couette flow in a minimal flow unit, Journal of Fluid Mechanics 538 (2005) 71-110.

(124) S. Ahuja, C. Rowley, I. Kevrekidis, M. Wei, T. Colonius, G. Tadmor, Lowdimensional models for control of leading-edge vortices: Equilibria and linearized models, in: 45th AIAA Aerospace Sciences Meeting and Exhibit, AIAA Paper, volume 709, p. 2007.

(125) D. Venturi, X. Wan, G. Karniadakis, Stochastic low-dimensional modelling of a random laminar wake past a circular cylinder, Journal of Fluid Mechanics 606 (2008) 339-368.

(126) J. Bailon-Cuba, J. Schumacher, Low-dimensional model of turbulent rayleighbénard convection in a cartesian cell with square domain, Physics of Fluids 23 (2011) 077101.

(127) J. Baggett, L. Trefethen, Low-dimensional models of subcritical transition to turbulence (1996).

(128) A. Bakshi, Analog Integrated Circuits, Technical Publications, 2009.

(129) C. H. K. Williamson, Defining a universal and continuous strouhal-reynolds number relationship for the laminar vortex shedding of a circular cylinder, Phys. Fluids 31 (1988) 2742-2744. 
(130) U. Fey, M. König, H. Eckelmann, A new Strouhal-Reynolds-number relationship for the circular cylinder in the range $47<R e<2 \times 10^{5}$, Phys. Fluids 10 (1998) $1547-1549$.

(131) L. Rayleigh, Aeolian tones, Philosophical Magazine XXIX (1915) 433-453.

(132) B. Ahlborn, M. L. Seto, B. R. Noack, On drag, Strouhal number and vortex-street structure, Fluid Dyn. Res. 30 (2002) 379Ü-399.

(133) P. Roushan, X. L. Wu, Structure-based interpretation of the Strouhal-Reynolds number relationship, Phys. Rev. Lett. 94 (2005) 054504.

(134) F. L. Ponta, H. Aref, Strouhal-Reynolds number relationship for vortex streets, Phys. Rev. Lett. 93 (2004) 084501.

(135) F. L. Ponta, Effect of shear-layer thickness on the Strouhal-Reynolds number relationship for bluff-body wakes, J. Fluids Struct. 22 (2006) 1133-1138. 


\section{APPENDICES}




\section{APPENDIX A. COPYRIGHT AGREEMENTS}




\section{A.1 Copyright statement for Chapter 1}

The picture from Figure 1.1 is reproduced from "DOE, Wind power today Federal wind program highlights, Technical Report, U.S Department of Energy, 2005." "Government information at DOE Web sites is in the public domain. Public domain information may be freely distributed and copied, but it is requested that in any subsequent use the Department of Energy be given appropriate acknowledgement".

Picture from Figure 1.1 can be found at the source below and the permission is available listed:

http://www.windpoweringamerica.gov/pdfs/2005_wind_power_today.pdf, under DOE-policies.

The picture from Figure 1.2 is reproduced from Wikimedia Commons, a freely licensed media file repository. Files are licensed under the Creative Commons Attribution-Share Alike 2.0 and/or 3.0 Unported license. According to these license, permission is granted to:

1. Share, copy, distribute and transmit the work.

2. Remix, adapt the work.

Under the following conditions:

1. Attribution You must attribute the work in the manner specified by the author or licensor (but not in any way that suggests that they endorse you or your use of the work).

2. share alike If you alter, transform, or build upon this work, you may distribute the resulting work only under the same or similar license to this one.

\section{ATTRIBUTIONS:}

Picture from Figure 1.2 was taken by Hans Hillewaert. Source and permissions availables at: http://en.wikipedia.org/wiki/File:Windmills_D1-D4_Thornton_ Bank.jpg, under CC-BY-SA-3.0. 
The picture from Figure 1.3 is reproduced from "S. Butterfield, W. Musial, J. Jonkman, P.Sclavounos, Engineering Challenges for Floating Offshore Wind Turbines, Technical Report, National Renewable Energy Laboratory (NREL), 2005." NREL is a national laboratory of the Department of Energy (DOE). "Government information at DOE Web sites is in the public domain. Public domain information may be freely distributed and copied, but it is requested that in any subsequent use the Department of Energy be given appropriate acknowledgement".

Picture from Figure 1.3 can be found at the source below and the permission is available listed:

http://www.nrel.gov/docs/fy07osti/38776.pdf, under DOE-policies. 
Excerpts of two published articles: "L. I. Lago, F. L. Ponta, L. Chen, Advances and trends in hydrokinetic turbine systems, Energy for Sustainable Development, 2010" and "L. Chen, F. L. Ponta, L. I. Lago, Perspectives on innovative concepts in wind-power, Energy for Sustainable Development, 2011" are reproduced in Chapter 1. The contract agreement with the publisher is included here, stating that permission was granted to reuse them.

\section{ELSEVIER LICENSE TERMS AND CONDITIONS}

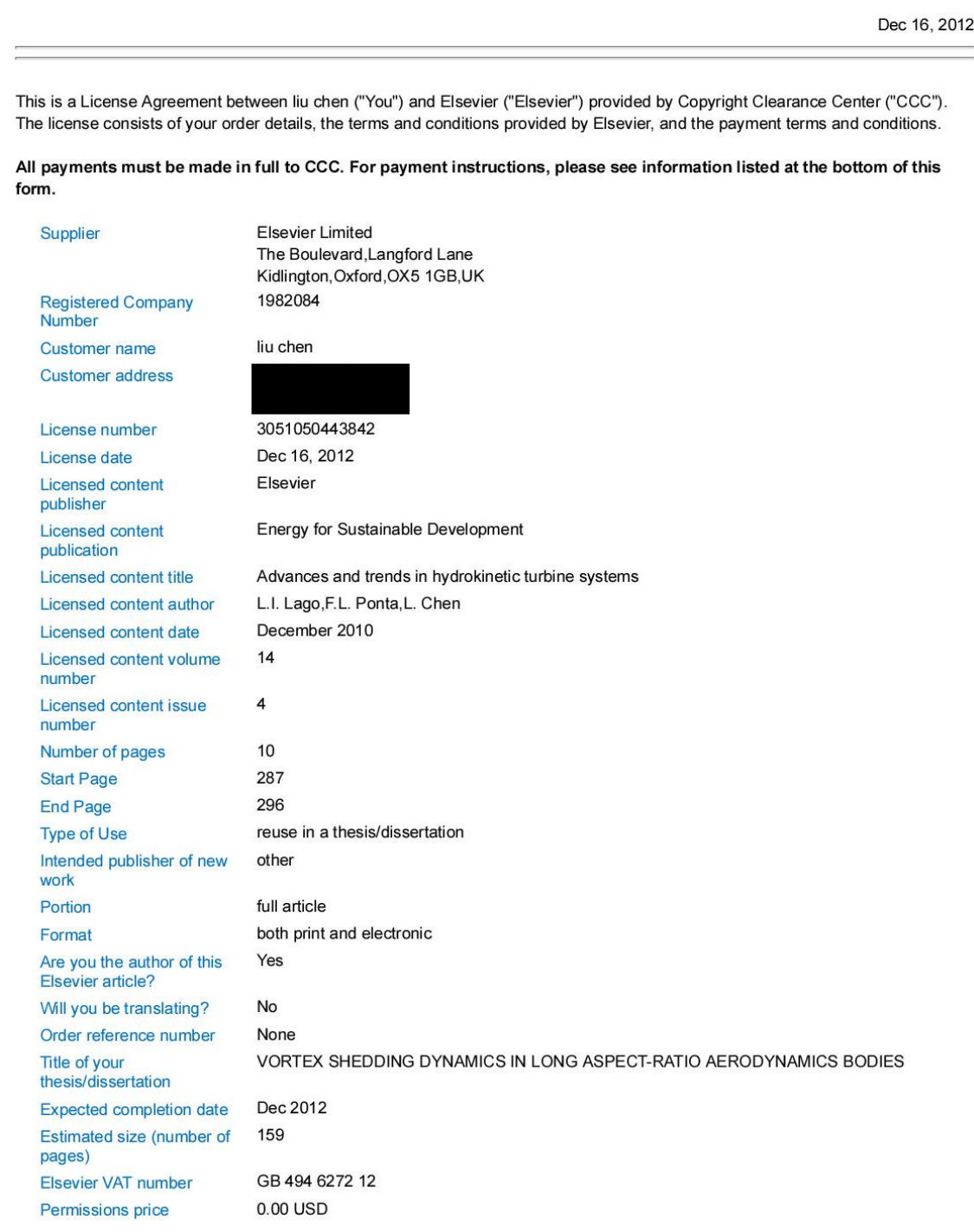




\section{ELSEVIER LICENSE TERMS AND CONDITIONS}

This is a License Agreement between liu chen ("You") and Elsevier ("Elsevier") provided by Copyright Clearance Center ("CCC"). The license consists of your order details, the terms and conditions provided by Elsevier, and the payment terms and conditions.

All payments must be made in full to CCC. For payment instructions, please see information listed at the bottom of this form.

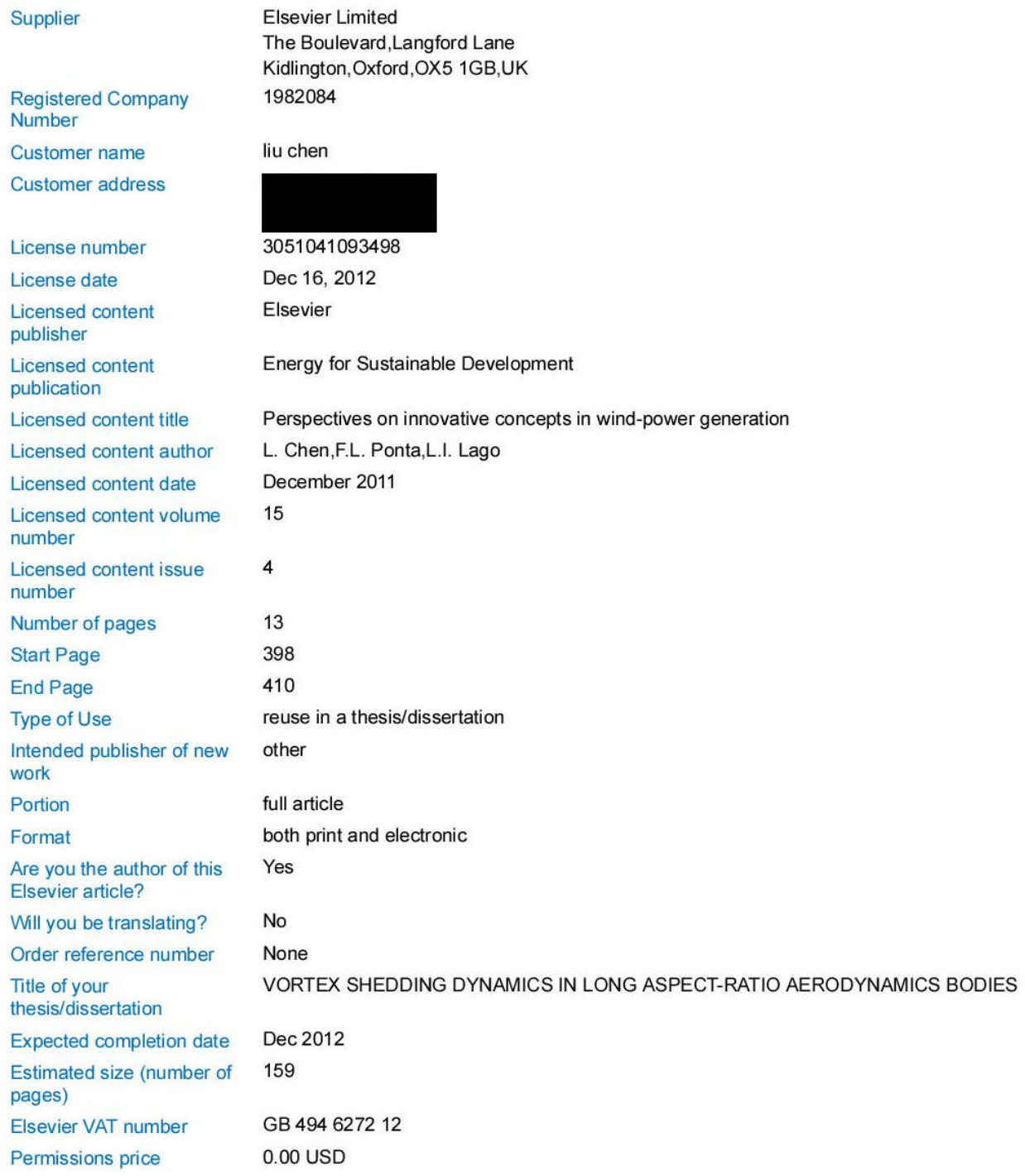

End Page $\quad 410$

3051041093498

Dec 16, 2012

Elsevier

Energy for Sustainable Development

Perspectives on innovative concepts in wind-power generation

L. Chen,F.L. Ponta,L.I. Lago

December 2011

15

4

398

reuse in a thesis/dissertation

other

full article

both print and electronic

Yes

No

None

VORTEX SHEDDING DYNAMICS IN LONG ASPECT-RATIO AERODYNAMICS BODIES

Dec 2012

159

0.00 USD 


\section{A.2 Copyright statement for Chapter 2}

Pictures from Figure 2.13 and upper image from Figure 2.16 are reproduced from "Numerical experiments on vortex shedding from an oscillating cylinder, Journal of Fluids and Structures, 2006". The contract agreement with the publisher is included here, stating that permission was granted to reuse the aforementioned figure.

\section{ELSEVIER LICENSE TERMS AND CONDITIONS}

Nov 20, 2012

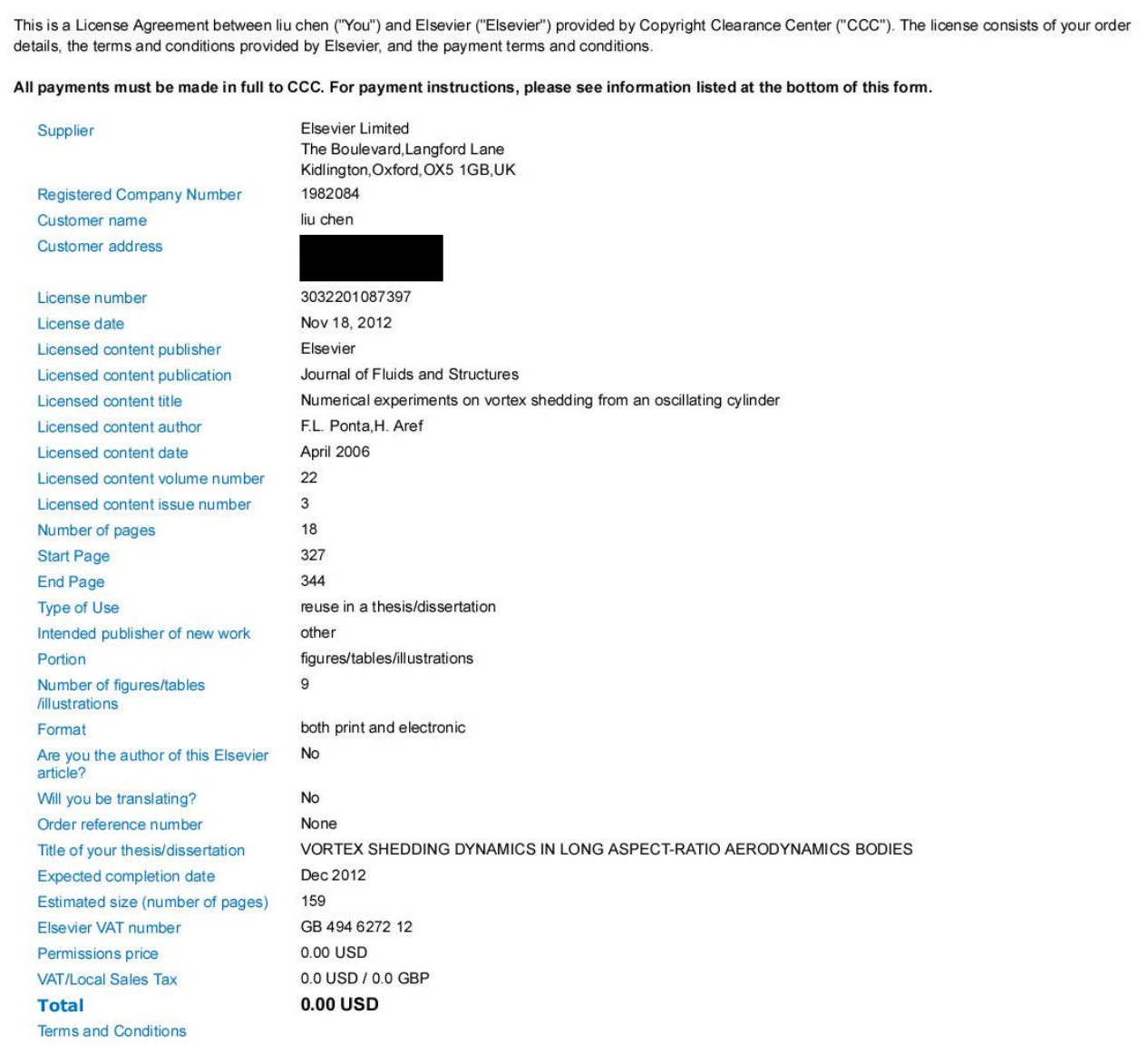


The background picture from Figure 2.13(synchronization regions from experimental results without the lines of different $\Delta$ in image (a) and dotted points in image (b)) and the picture from Figure 2.15(a) are reproduced from "C. H. K. Williamson, A. Roshko, Vortex formation in the wake of an oscillating cylinder, Journal of Fluids Structure,1988". The contract agreement with the publisher is included here, stating that permission was granted to reuse the aforementioned figures.

\section{ELSEVIER LICENSE TERMS AND CONDITIONS}

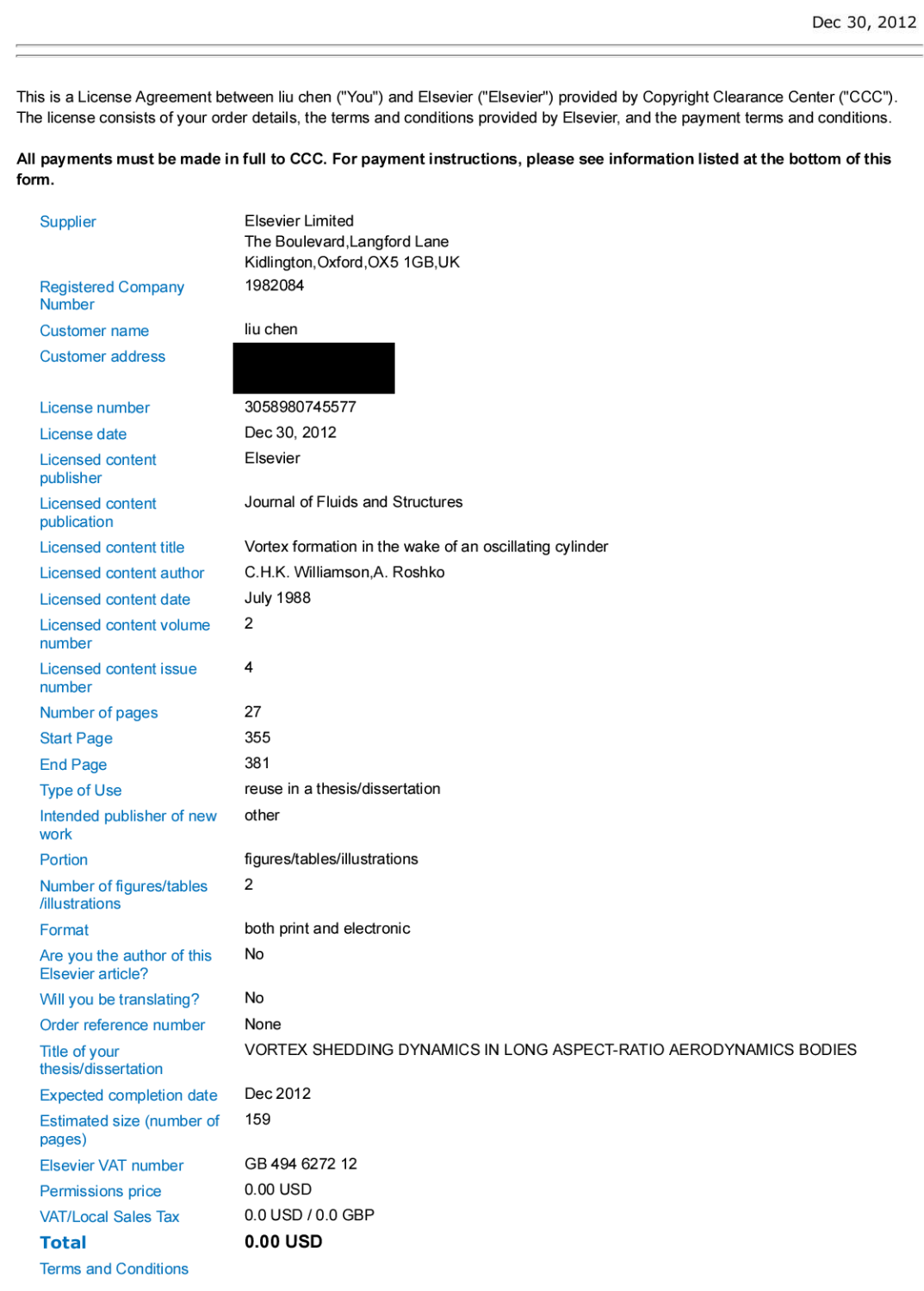

